Propositions

1. Whether or not compounds activate $\mathrm{AhR}$ in vitro, does not say anything about their physiological relevance in vivo.

(this thesis)

2. Microbial metabolites are more important for intestinal health than the (non-pathogenic) gut microbiota.

(this thesis)

3. In science, deductive and inductive reasoning are equally important and should be combined to build new knowledge.

4. For healthy individuals, personalised nutrition is overrated.

5. Multidisciplinary research opens doors but covers deep lakes.

6. If a sneeze can set off a fire alarm, safety is not assured.

Propositions belonging to the thesis, entitled

The gut way to health: in vitro studies on immunomodulatory food compounds Jonna Koper

Wageningen, 14 February 2020 


\section{The gut way to health:}

In vitro studies on

immunomodulatory food compounds

Jonna Koper 


\section{Thesis committee}

\section{Promotors}

Prof. Dr V. Fogliano

Professor of Food Quality and Design

Wageningen University \& Research

Prof. Dr J.M. Wells

Professor of Host-Microbe Interactomics

Wageningen University \& Research

\section{Co-promotors}

Dr E. Capuano

Assistant professor, Food Quality and Design

Wageningen University \& Research

Dr L.M.P. Loonen

Researcher, Host-Microbe Interactomics Group

Wageningen University \& Research

\section{Other members}

Prof. Dr E. Kampman, Wageningen University \& Research

Prof. Dr H. Smidt, Wageningen University \& Research

Dr T. Smokvina, Danone Research, Orsay, France

Dr P. Jeurink, FrieslandCampina, Wageningen

This research was conducted under the auspices of the Graduate School VLAG (Advanced studies in Food Technology, Agrobiotechnology, Nutrition and Health Sciences). 


\title{
The gut way to health: In vitro studies on immunomodulatory food compounds
}

\author{
Jonna Koper
}

Thesis

submitted in fulfilment of the requirements for the degree of doctor

at Wageningen University

by the authority of the Rector Magnificus,

Prof. Dr A.P.J. Mol,

in the presence of the

Thesis Committee appointed by the Academic Board

to be defended in public

on Friday 14 February 2020

at 1:30 p.m. in the Aula. 
Jonna Koper

The gut way to health: in vitro studies on immunomodulatory food compounds, 166 pages.

PhD thesis, Wageningen University, Wageningen, The Netherlands (2020) With references, with summary in English

ISBN 978-94-6395-195-1

DOI https://doi.org/10.18174/505381 


\section{Table of contents}

\section{Chapter 1}

General introduction

\section{Chapter 2}

Studies on the in vitro fermentation of pectin and immune-stimulatory mechanism

of pectin-derived linear arabinans on human dendritic cells

\section{Chapter 3}

Polyphenols and tryptophan metabolites activate the Aryl hydrocarbon Receptor in

an in vitro model of colonic fermentation

\section{Chapter 4}

Tryptophan supplementation increases the production of microbial-derived AhR agonists in an in vitro simulator of intestinal microbial ecosystem

\section{Chapter 5}

Aryl hydrocarbon Receptor activation during in vitro and in vivo digestion of broccoli

(brassica oleracea) after different cooking methods

\section{Chapter 6}

General discussion

\section{Appendix}

Summary

149

Acknowledgements

About the author 


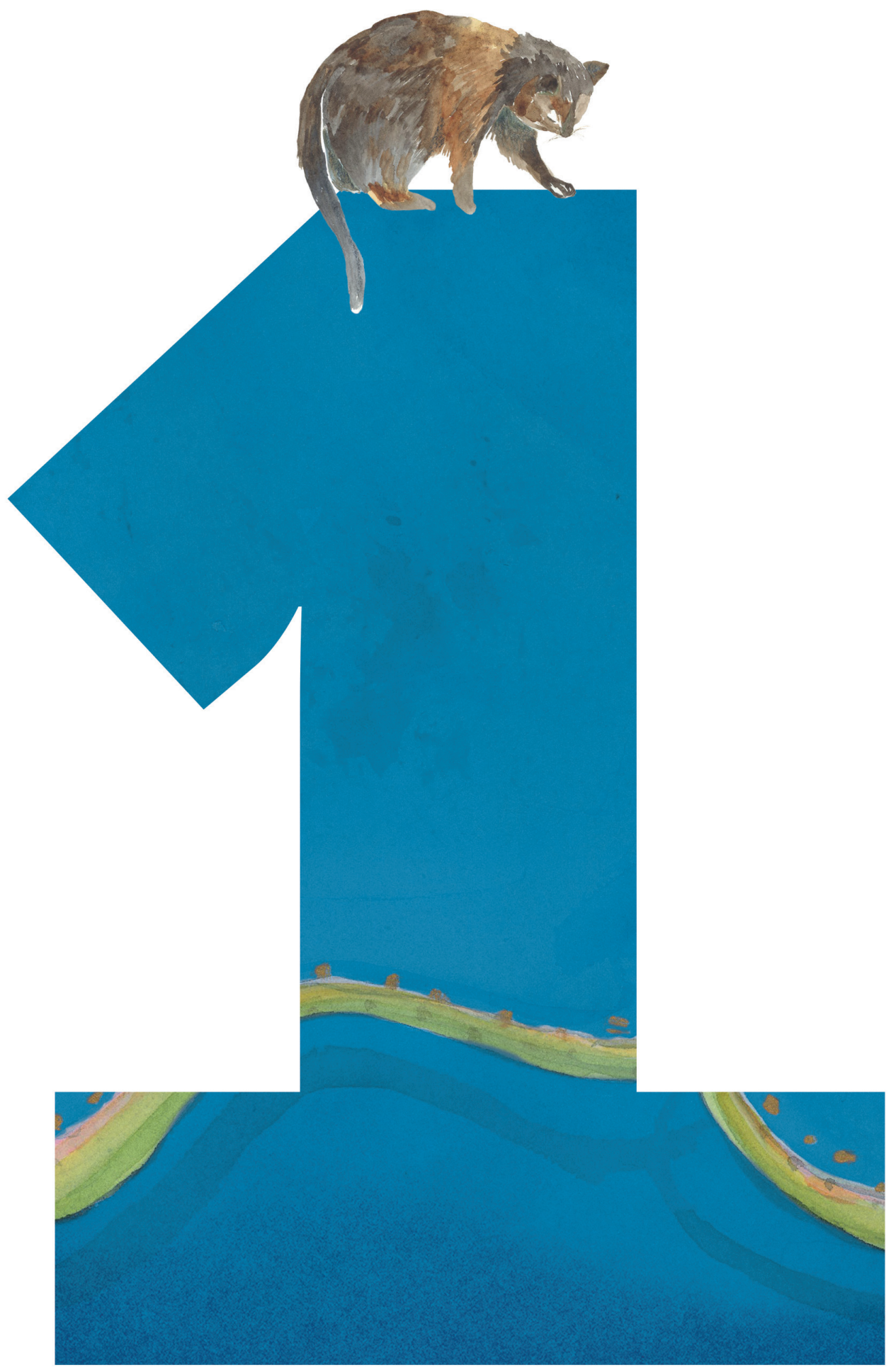




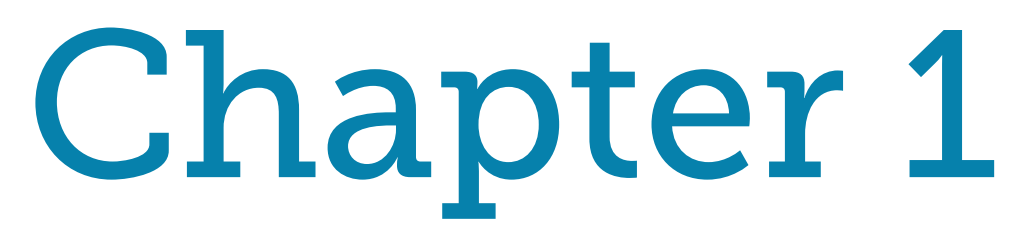

General introduction 



\section{Introduction to the thesis}

Recent insights have shown that there is a triangular relationship between the diet, immune system and intestinal microbiome, in which the gut microbiota is a substantial contributor to human metabolism and health ${ }^{1}$. Therefore, increased interest exists in the relationship between the fields of nutrition, immunology and microbiology. Not only can the diet influence the composition of the gut microbiota, but the microbiota and its products can, in turn, also have profound effects on the biology of the host ${ }^{2-4}$. However, the relationship between microbiota and health is highly complex and does not only require understanding of specific microbial interactions via metabolites or other factors (including the pathogenic traits of pathobionts) but also detailed knowledge on host physiology, in particular intestinal homeostasis and mucosal immunity ${ }^{5}$ (Figure 1). The fraction of food that is not digested and absorbed by the host, serves as nutrients for the microbiota. Despite progress in predicting the effects of fibres on microbial composition and short-chain fatty acid (SCFA) production ${ }^{1,6}$, we do not yet understand the full complexity of dietary effects on the microbiota and keystone species that play a major role in shaping individual microbiota ecosystems. This understanding is a critical step in developing rational therapeutic interventions to modify microbiota composition and activity to promote health and prevent or treat diseases.

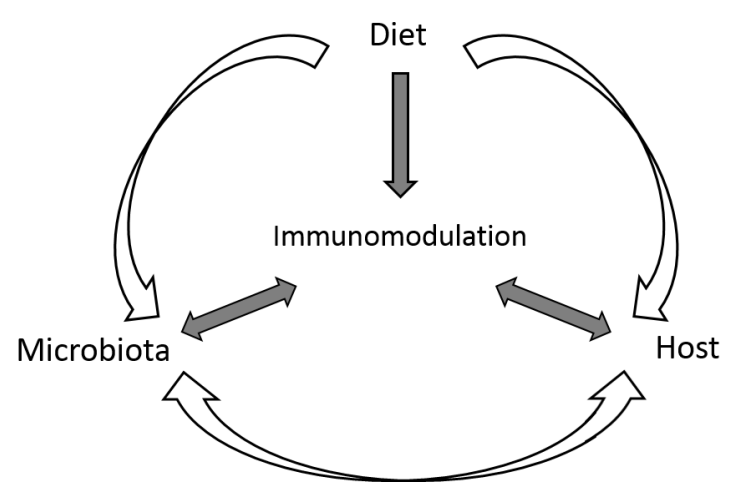

Figure 1. Schematic overview of the contribution of diet to the interactions between microbiota and host, and its impact on immunomodulation.

Previous studies showed that a high fibre diet resulted in a different microbiota composition and increased production of SCFAs than in a diet low in fibres ${ }^{1,6}$. This was the first evidence of the relationship between diet and microbiome. Dietary changes towards a Western style diet, rich in fat and protein and low in fibres, fruit and vegetables, lead to an increased risk for chronic immune diseases such as inflammatory bowel disease (IBD), allergies and autoimmune 
diseases ${ }^{1,7,8}$. Microbial fermentation of complex carbohydrates that are not digested by the host in the small intestine, results in the production of SCFAs, which are known to directly and indirectly affect the immune system ${ }^{9-11}$. Another class of bacterial metabolites that impact on intestinal homeostasis and immunity are the tryptophan metabolites, which can activate the Aryl hydrocarbon Receptor $(A h R)^{12}$.

A better understanding of the relationship between diet, including food processing, and the activity of metabolites produced by the gut microbiota and their effect on the host, is an essential step in designing food that benefits human health. The nutrient composition of a diet is obviously of importance to health, but also the way food is designed plays an important role in how much reaches compounds the colon where it is metabolised by the microbiota ${ }^{13}$. Food design, both industrial food processing and domestic food preparation, can therefore be a tool to influence the nutrients that reach the lower gut and impact intestinal health. However, more insight is needed into the modulatory effect of digestion and microbial fermentation on the interactions between dietary components and the gut immune system. Therefore, this thesis describes the effects of different dietary components (fibre, tryptophan, glucosinolates and polyphenols) on immune modulation, mainly focussing on activation of the Aryl hydrocarbon Receptor (AhR), during human digestion and fermentation. The results described in this thesis give insight into the effects of food preparation and microbial fermentation on the potential health properties of food compounds. 


\section{Immunomodulation: effects of dietary fibre and microbiota on the host}

The mammalian immune system is complex, having multiple systems and mechanisms to protect the host from invading microbes, parasites and viruses. In the intestine, various types of cells, such as innate and adaptive immune cells and epithelial cells, are involved in the gut immune system ${ }^{14}$. Together, all immune cells related to intestinal associated lymphoid tissue are about the size of the brain ${ }^{15}$, reflecting their importance in human biology. The human intestine harbours more than $10^{15}$ commensal bacteria, most of which are located in the large bowel. The microbiota has numerous symbiotic functions including vitamin production, maximising energy harvest, host immune functioning, tissue development and colonisation resistance against pathogens ${ }^{1,16,17}$. Dietary compounds can modulate the immune system either directly as parent compounds or indirectly by metabolites formed from the parent compounds. Indirectly, dietary fibres, i.e. components of plant cell walls that are resistant to human digestion ${ }^{18,19}$, are fermented by microbiota in the colon to produce SCFA. SCFAs have important effects on health through various mechanisms ${ }^{20-22}$, and play a role in gut homeostasis and induction of colonic T-regulatory cells ${ }^{23}$. Additionally, SCFAs can lower the colonic $\mathrm{pH}$, thereby inhibiting pathogenic growth ${ }^{24}$. Dietary fibres are also utilised as prebiotics to promote the growth of specific genera and species of the intestinal microbiota ${ }^{25,26}$.

Apart from their indirect effects on the microbiota and SCFA production, several fibres have been reported to have immune-stimulatory effects on immune cells ${ }^{27}$. The mechanisms involved in immunomodulatory effects of fibres are poorly understood with the exception of $\beta$-1,3-linked glucans, which are components of the cell walls of fungi. These glucans signal through binding to the C-type lectin receptor (CLR) Dectin-1, which is important for antifungal immunity ${ }^{28-30}$. Other fibres that are reported to have immune-stimulatory effects are pectin-derived arabinans, like linear or branched arabinan ${ }^{27}$. Pectins are the main compound of the middle lamella in the plant primary cell wall, and consist of homogalacturonan (HG), rhamnogalacturonan I and II (RG I and II) and xylogalacturonan (XGA) ${ }^{31}$. Arabinans are the side chains of RG I and were able to directly bind mouse dendritic cells (DC ${ }^{27}$. In this thesis, both direct and indirect effects by dietary compounds on immune modulation were investigated. 


\section{Diet-microbiota influence on the AhR-immune axis}

Another important connection between host, diet and microbiota is the AhR-immune axis. AhR is a cytosolic receptor expressed by many cells in the human body and previously known to bind, amongst others, 2,3,7,8-tetrachlorodibenzo-p-dioxin (TCDD), contributing to its detoxification ${ }^{32}$. However, recent studies have demonstrated that AhR also participates in the establishment and maintenance of intestinal homeostasis, including maintaining epithelial barrier integrity, protection from pathogens and regulation of commensal gut microbiota ${ }^{12,16,33}$. The diet can influence AhR-dependent mechanisms by providing a source of potential AhR ligands. In addition, the microbiota can produce AhR ligands through metabolism of tryptophan. AhR can be considered as an important anti-inflammatory receptor which combines dietary, microbial, metabolic and endogenous signals to activate immune response and alter the gut microbiota composition ${ }^{1}$. AhR is expressed by a number of immune cells, and thus, AhR signalling provides a molecular pathway that integrates the effects of the environment and metabolism on the immune response ${ }^{34}$.

\subsection{AhR pathway}

AhR consists of a basic helix-loop-helix (bHLH) protein belonging to the Per-Arnt-Sim (Pas) family ${ }^{35}$. The receptor resides in the cytosol and it is ligand-activated. Several domains and regions are functionally important within the AhR protein complex. At the amino terminal part, the bHLH-Pas region is a key player in the interaction with hsp90, binding of DNA and dimerisation with AhR nuclear translocator (Arnt). Arnt is also involved in translocation of the receptor to the nucleus, involving a nuclear localisation signal (NLS) and a nuclear export signal (NES). Additionally, AhR has a Pas domain, which contains two structural repeats: Pas A and Pas B. While Pas A is involved in the dimerisation of AhR with Arnt, Pas B binds the ligands and interacts with hsp90. The Pas domain is not only important at the amino terminal, but also at the carboxy terminal part where it is involved in the interaction of AhR with XAP2, an AhR associated protein. The carboxy terminal consists of a transactivation domain with three subdomains. The total AhR has a size of 95 to 125 kDa, varying amongst different species ${ }^{36-38}$.

When AhR ligands enter the cell, they pass the plasma membrane and bind the receptor ${ }^{39}$ (Figure 2). At that moment, AhR is present as an inactive complex consisting of multiple proteins and resides in the cytosolic compartment. It contains 2 hsp90 molecules: XAP2 and P2340-42. Hsp90 in the cytoplasm has the function to localize AhR in the absence of a ligand, where it protects its degradation and transformation. XAP2 minimises the degradation of AhR and plays a role in the regulation of the translocation of AhR to the nucleus. Moreover, it boosts AhR signalling when it is joined with hsp90 ${ }^{43,44}$. P23 is involved in the stabilisation of the AhR complex ${ }^{45}$. 
Upon ligand binding to AhR, the receptor changes its conformation, exposes the NLS and enters the nucleus. After being translocated into the nucleus, AhR binds Arnt and subsequently binds to dioxin or xenobiotic responsive elements (DRE or XRE), which are target gene promoters to induce expression of AhR-responsive genes such as CYP1A1 and CYP1b1 ${ }^{46}$. As a result, the transcription of adjacent genes is activated, one of which is IL-22 $2^{47-49}$. This pathway interacts with some other pathways, for example the estrogen, NF-kB and cAMP pathway ${ }^{1,50}$. As a result of the transformation and activation events, a variety of proteins are expressed, including drugmetabolising enzymes.

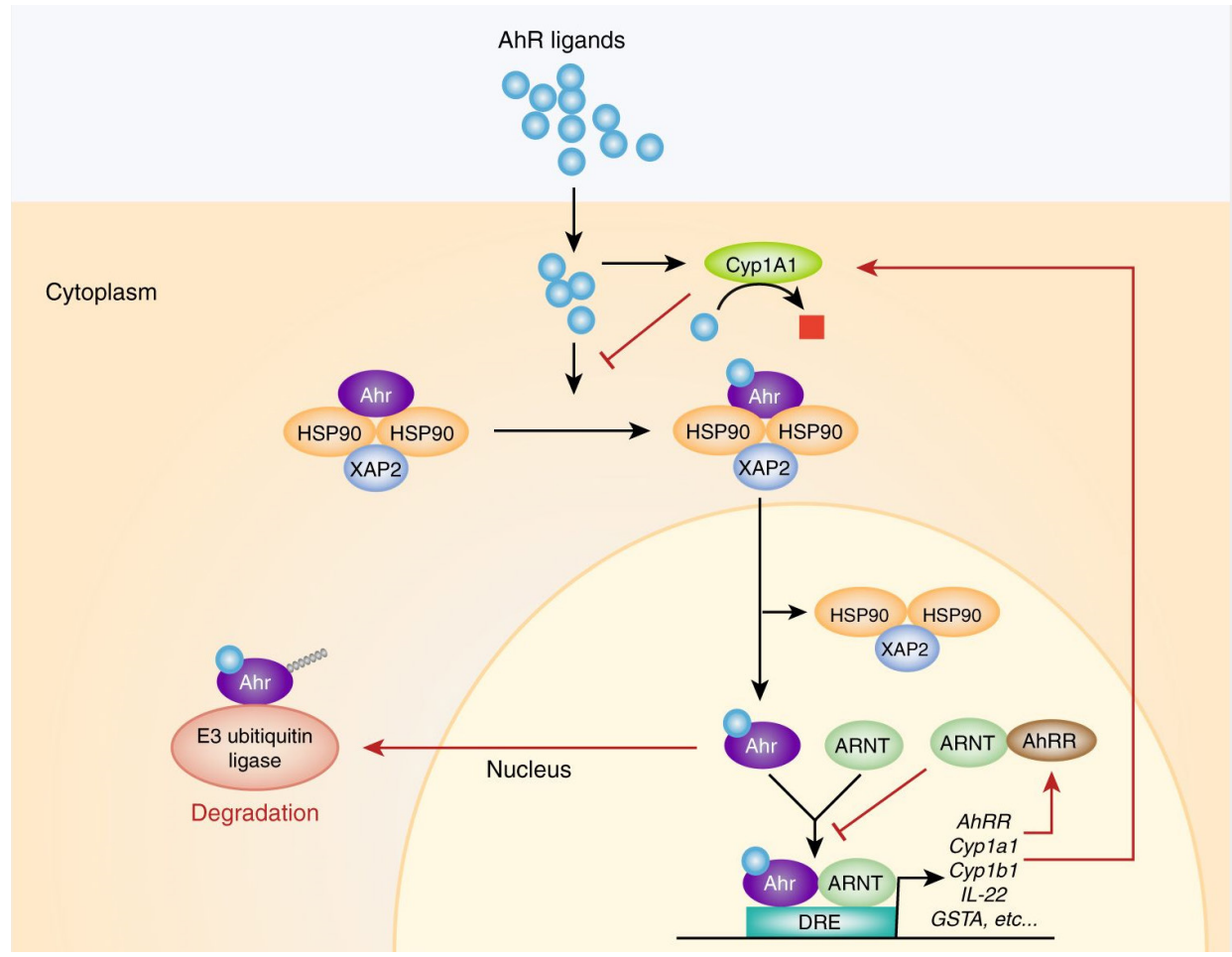

Figure 2. AhR signalling pathway. Aryl hydrocarbon Receptor (AhR), AhR repressor (AhRR), AhR nuclear translocator (Arnt), cytochrome P450 1A1 (Cyp1a1), cytochrome P450 1B1 (Cyp1b1), dioxin response element (DRE), glutathione-S-transferase A (GSTA), heat shock protein 90 (hsp90), interleukin 22 (IL-22), HBV X-associated protein 2 (XAP2). Adapted from Lamas et al. ${ }^{39}$.

\subsection{AhR activation by ligands}

AhR is known to mediate the toxic effects of halogenated aromatic hydrocarbons (HAHs). Apart from HAHs, a number of ligands for the AhR have been reported in the scientific literature, including exogenous diet-derived compounds such as polycyclic aromatic hydrocarbons (PAHs), aromatic amines and phytochemicals, as well as endogenous bacterial metabolites (Figure 3) 1,51. 
The main exogenous ligands for AhR are derived from edible plant tissues, i.e. vegetables, fruits, teas and herbs ${ }^{32,35}$. Several studies showed that especially polyphenols can exert an agonistic or antagonistic effect on AhR transduction pathways ${ }^{52-58}$. Moreover it is stated that at physiological conditions, polyphenols are antagonists rather than agonists, as the agonistic effects induced by polyphenols often require higher concentrations ${ }^{52,58-62}$.

Besides food derived compounds, also endogenous microbial metabolites can act as AhR ligands. Examples of microbial metabolites that can activate AhR are tryptophan metabolites from e.g. Lactobacilli12, microbial pigments phenazines and naphthoquinone produced by respectively Pseudomonas aeruginosa and Mycobacterium tuberculosis, and 1,4-dihydroxy-2-naphthoic acid, a vitamin K2 precursor, metabolised by Propionibacterium freudenreichii ${ }^{1}$. Additionally, AhR senses bacterial pigments, thereby controlling some antibacterial responses ${ }^{63}$.

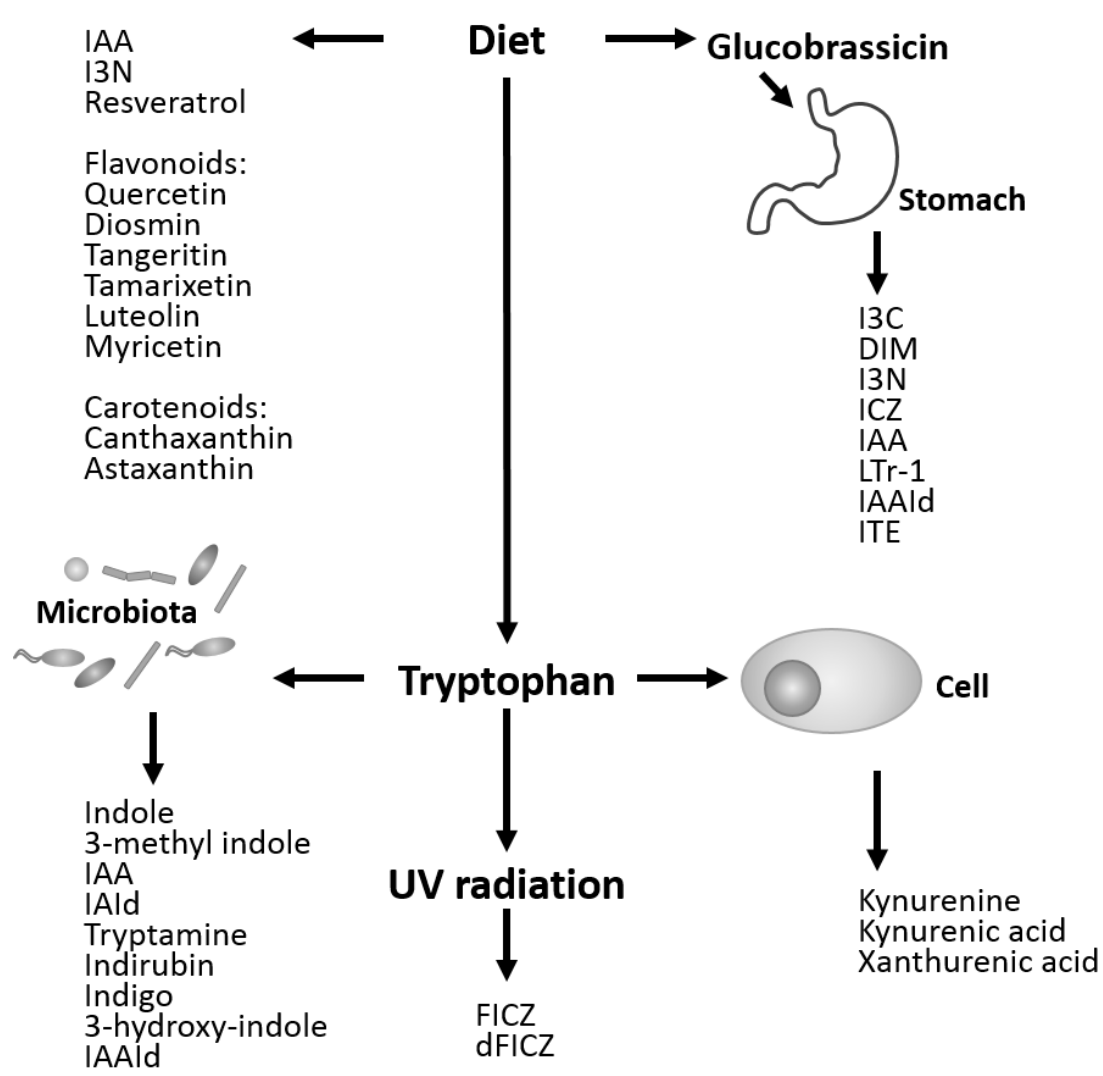

Figure 3. Several identified AhR ligands, originating from diet, tryptophan metabolism, or cell metabolism. Adapted from Lamas et al. ${ }^{39}$. 
AhR activation can be measured in different ways, for example in vivo by using mice models comparing AhR ${ }^{-1}$ with wild type mice, or by dietary intervention studies with AhR ligands and measuring gene expression of AhR or the downstream CYP1a1 gene. AhR activation can also be measured in vitro, by using various cell lines. In this thesis we used two different in vitro cell lines, first the CALUX cell line (BioDetection Systems), where rat liver cells (H4LucO3) were transfected with the pGudLuc1.1 plasmid luciferase gene and responsiveness is conferred by DRE sequences ${ }^{64}$. In our last study (Chapter 5), we used the recently developed human Hep-G2 Lucia AhR reporter assay (Promega), which has the same principle as the CALUX cells, but in human liver cells.

In this thesis, we investigated the fate of dietary AhR ligands during digestion and colonic fermentation. Results lead to knowledge on how to increase the delivery of potential AhR ligands to the lower gut and the potential role of food in the establishment and maintenance of a healthy gut. In the next sections, the most important classes of diet-derived AhR ligands (polyphenols, glucosinolates and tryptophan metabolites) are discussed in more detail.

\subsubsection{Polyphenols}

Polyphenols are secondary metabolites from plants and have been widely studied for their antioxidant properties to inhibit oxidation and protection against reactive oxygen species ${ }^{65,66}$. Recently it became clear that polyphenols are active in several other mechanisms and the health effects of polyphenols include more complex interactions with the host, including anti-inflammatory and antimicrobial capacities ${ }^{66,67}$. Polyphenols can be grouped based on their chemical structure, i.e. the number of phenol (aromatic) rings and the way the rings are connected in phenolic acids, flavonoids and non-flavonoid polyphenols (stilbenes and lignans)68. Several studies investigated the capacity of individual polyphenols to activate $A h R^{32,62,69-71}$. These showed that mainly flavonoids are potent AhR ligands. Furthermore, the number of hydroxyl groups and planar conformation are important factors that determine the AhR activation capacity ${ }^{72}$.

Flavonoids consist of a basic core structure with a C6-C3-C6 flavone skeleton. The C3 portion of this skeleton is often cyclised with an oxygen molecule and can vary in location of unsaturation and oxidation ${ }^{73}$. At least 5000 different plant-derived flavonoids have been discovered and isolated from a wide variety of plants ${ }^{74,75}$. Flavonoids can be further divided into six groups: flavonols, flavones, flavanones, flavanols, isoflavones and anthocyanidins ${ }^{68}$. The degree of hydroxylation, substitutions, conjugations and degree of polymerisation determines the chemical nature and therefore the structural class of flavonoids. Often these occur in the plant as glycosides ${ }^{76}$. The biochemical activity of flavonoids depends on the chemical structure and relative orientation of the molecule. Naturally, flavonoids are found in almost all parts of plants, and are particularly abundant in the external tissues like coats or skin. Therefore, flavonoids 
form an integral part of the human $\operatorname{diet}^{76}$. Depending on their size and structure, polyphenols can be absorbed in the small intestine but the bioavailable fraction is typically small (approximately $5-10 \%)$. Most of the polyphenols reach the colon unchanged where they are fermented by the gut microbiota ${ }^{66,67}$. In the colon, polyphenols are converted into low molecular weight phenolic metabolites by cleavage of glycosidic linkages and breakdown of the heterocyclic backbone of the resulting aglycon, thereby facilitating absorption in the gut (Figure 4) 66,67 .

\section{Small intestine}

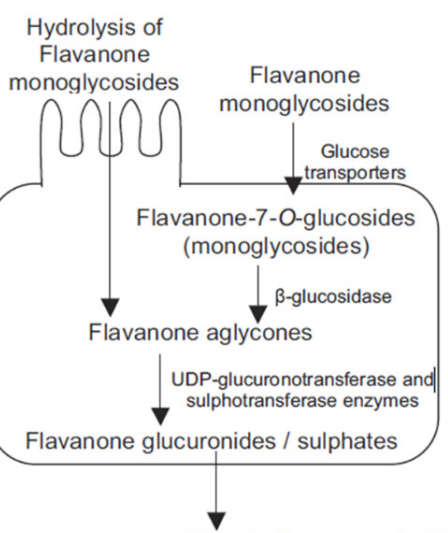

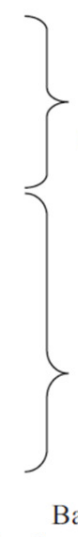

Basolateral side

\section{Colon}

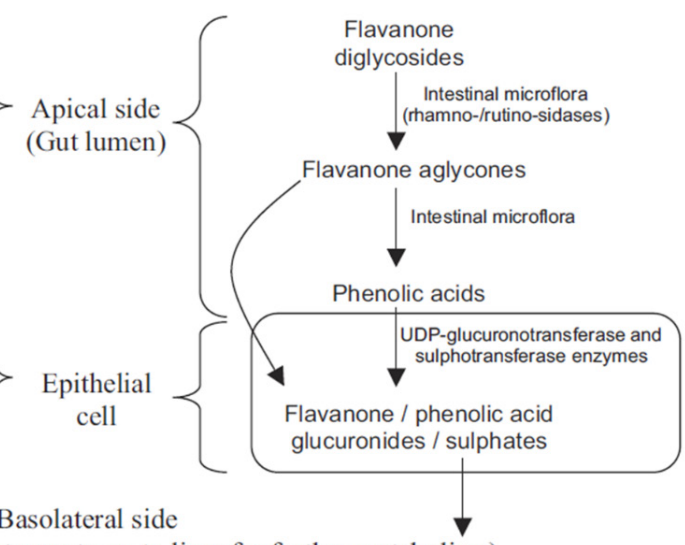

(Metabolites enter in blood stream to go to liver for further metabolism)

Figure 4. Schematic presentation of flavanone metabolism in the small and large intestine as an example of polyphenols fate in the human superorganism ${ }^{66}$.

\subsubsection{Glucosinolates}

Beneficial health effects of vegetables of the genus Brassica (e.g. broccoli and cabbage) have been partly linked to glucosinolates (GLS), which are secondary metabolites ${ }^{77}$. The GLS structure consists of a $\beta$-D-thioglucose group, a sulfonated oxime group, and a side chain derived from amino acids ${ }^{78}$. Over 130 GLS have been identified and these can be divided into three classes: indole GLS, aliphatic GLS and aromatic GLS. Brassica vegetable tissue contains myrosinase and high amounts of glucosinolates. Myrosinase, an endogenous plant enzyme, can hydrolyse glucosinolates into an array of breakdown products with a variety of physiological effects ${ }^{78}$.

The study of Hubbard et al., demonstrated that the beneficial aspects of dietary broccoli (Brassica oleracea) upon intestinal health are associated with an increase in AhR activation ${ }^{79}$. When glucobrassicin, a GLS, is hydrolysed, compounds such as isothiocyanates (ITC) are formed and those can be converted into AhR ligands ${ }^{16,80-82}$. Glucosinolates and myrosinase are physically 
segregated from each other in intact vegetable tissues. Food preparation and chewing of the vegetables results in the loss of compartmentalisation in the vegetable tissue, and glucosinolates and myrosinase can come into contact with each other, which catalyses the enzymatic hydrolysis of glucosinolates ${ }^{83}$. One of the degradation products of enzymatic hydrolysis of glucobrassicin is indole-3-carbinol (I3C). I3C exhibits several biological functions, including reduction of DNAadduct formation, induction of apoptosis and inhibition of tumour growth ${ }^{84,85}$. I3C is unstable and can undergo acid-catalysed oligomerisation at low $\mathrm{pH}$, for example in the stomach. The oligomerisation of I3C results in a mixture of products, like 2-(indol-3-yl methyl)-indol-3-yl] indol-3-methane (LTr-1), indolo[3,2-b]carbazole (ICZ) and 3,3-diindolylmethane (DIM) ${ }^{86}$. I3C is a ligand of the AhR and can bind weakly to the receptor, whereas the products of the acidcatalysed oligomerisation have a higher affinity for this receptor ${ }^{87}$.

\subsubsection{Tryptophan}

Tryptophan (Trp) is an essential amino acid and abundant in protein-rich foods like beans and nuts, cheese, meat, fish, and eggs ${ }^{88}$. The daily intake of Trp in the average Western diet is approximately 600 - 900 mg $^{89}$, of which 70 - $95 \%$ is absorbed depending on the source. Of the absorbed fraction, around $30 \%$ is used for energy 89,90 and the remaining Trp can be metabolised in the body by endogenous enzymes indoleamine 2,3-dioxygenase (IDO) and

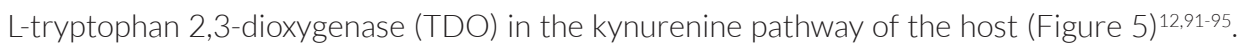
Furthermore, Trp is a precursor in the serotonin and melatonin pathways ${ }^{94,96}$. A fraction of Trp ends up in the skin, where it can be converted into 6-formylindolo[3,2-b]carbazole (FICZ) by UVB irradiation. FICZ can also be formed by a reaction between $\operatorname{Trp}$ and $\mathrm{H}_{2} \mathrm{O}_{2}$, rearrangement of indole-3-acetaldehyde (IAAId) and by yeasts present on the skin (Malessezia species) ${ }^{97}$. FICZ has a high binding affinity for AhR, but undergoes rapid degradation, so its effects on the host may be limited ${ }^{98,99}$.

The fraction of Trp that escapes absorption ends up in the colon and can there be metabolised by the gut microbiota into AhR active indole derivatives. The bacteria Lactobacillus reuteri ${ }^{12,100}$, L. murinus and L. taiwanensis ${ }^{100}$, and Clostridium sporogenes ${ }^{101,102}$ can all produce indoles from Trp. Lactobacilli are able to produce the AhR ligands indole-3-acetic acid (IAA) ${ }^{100}$ and indole3-aldehyde (IAId) ${ }^{12}$. The C. sporogenes metabolise Trp into indole-3-propionic acid (IPA) ${ }^{101}$ and 3-methyl indole (or skatole) ${ }^{102,103}$. IPA is able to stimulate the AhR but not in a dose-dependent manner while 3-methyl indole can activate the AhR in a dose-dependent manner ${ }^{103}$. After absorption by the colon epithelium, the Trp metabolites may be further metabolised in the host tissues. For instance, indole can be metabolised into oxindole by mammalian oxidases ${ }^{104}$. Oxindole (or 2-oxindole) and also indole itself were shown to activate AhR in a dose-dependent manner ${ }^{105}$. 


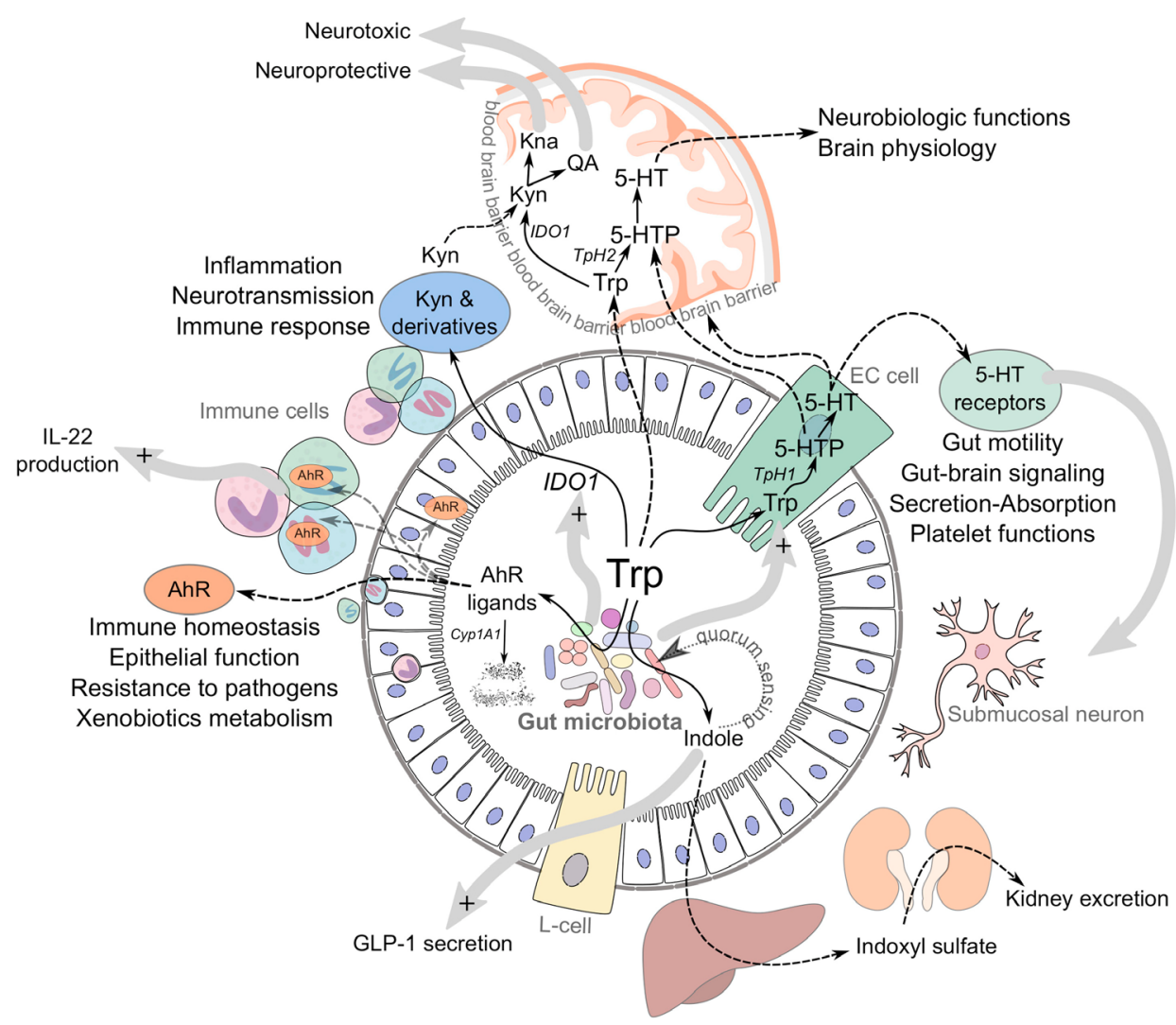

Figure 5. Tryptophan metabolism. Trp that is absorbed in the small intestine is used for energy, or metabolised into serotonin or kynurenine. The remaining Trp is metabolised by the microbiota. Adopted from Agus et al. 95 .

\subsection{Gut microbiota and AhR}

As stated before, the intestinal immune system comes into contact with many antigens, such as from pathogens, food and commensals. Various species of the gut microbiota have been shown to influence metabolic pathways in human hosts ${ }^{106}$. For instance, Clostridium and Eubacterium genera are known to metabolise phenolic compounds into bioactive metabolites that are taken up by the host ${ }^{107}$. Furthermore, the intestinal microbiota produces metabolites that can induce an immune response. It was suggested that Trp metabolites play a role in the immune response via AhR. In line with this, it has been shown that culture supernatants of Trp metabolising L. reuteri and Allobaculum species activate $A h R^{100}$. When using Trp as the major energy source in the diet of mice, the relative abundance of Lactobacilli producing indole-3-aldehyde increased. This resulted in a higher production of IL-22, which in turn affected the microbiota and provided resistance against colonisation with Candida albicans and consequently gut inflammation ${ }^{108}$. 
Zelante et al. showed that L. reuteri can produce IAAId, an AhR agonist, showing an additional manner in which microbiota can contribute to AhR stimulation ${ }^{12}$. Takamura et al. showed that certain heat-killed Lactobacillus strains activated the AhR using an AhR reporter assay, but whether this was the bacterium itself or its metabolites remained unclear ${ }^{108}$.

It was demonstrated that a divergence in microbiota composition between AhR genotypes in mice resulted in differences in metabolite abundance and host gene expression. 16S rDNA gene sequencing was performed to find differences in microbiota between $\mathrm{AhR}^{-/+}$and $\mathrm{AhR}^{-/-}$ mice $^{109}$. There was no significant difference between Bacteroidetes and Firmicutes, the two most dominant phyla in mice and humans, but there were significant changes in the other phyla abundance. This suggests that AhR expression influences the composition of the microbiota, but only the lower abundant phyla. Overall, results obtained in multiple studies indicate that AhR plays a role in maintaining the balance in the microbiome, possibly indirect through the effect on the IL-22 pathway ${ }^{12,108-110}$. 


\section{In vitro fermentation}

In order to study the relationship between host, diet and the microbiome, fermentation studies are necessary. However, in vivo studies in humans are expensive, time-consuming and ethically restricted, thereby limiting research possibilities. Furthermore, sampling of specific locations along the digestive tract comes with practical difficulties. Another option would be to use animal models, in which for example rats or pigs can be used as a model for humans. However, animal models require ethical justification and 'live' sampling during digestion and fermentation is practically very challenging. Furthermore, intrinsic differences exist between human and murine core gut microbiota, which bring into question the capability of mouse models to recapitulate effects of diet on the human gut microbiota ${ }^{111}$. As an alternative to animal experiments several in vitro fermentations models have been developed, in which fermentation with human faecal microbiota can be mimicked ${ }^{112}$.

Static (or batch) fermentation models use a closed anaerobic vessel incubated at $37^{\circ} \mathrm{C}$ and are inoculated with faecal sample, microbial growth medium and the substances to be fermented ${ }^{113}$. It is a simplified fermentation method, high throughput and relatively inexpensive. Fermentation time can be up to 48 hours, and generally no new substrate is added. Dynamic fermentation models are more complex, can be long term and may comprise of multiple connected vessels kept within a specific $\mathrm{pH}$ range and fixed volume. Several dynamic models exist ${ }^{114,115}$, for example, the TNO In Vitro Model of the Colon (TIM-2), the Dynamic Gastrointestinal Simulator (SIGMI) and the Simulator of the Human Intestinal Ecosystem (SHIME), which was used in this thesis.

The main advantage of the SHIME model is that it is possible to study the effects of long-term feeding on the microbiota, over several weeks. Another advantage is that the conditions found in each main segment of the colon are mimicked in different vessels. Samples can be taken at any time point and location during digestion, with the major advantage being the separation of the colon segments. A limitation is the lack of nutrient or compound absorption, lack of peristaltic movements and the semi-static nature of the system (Chapter 6, General discussion). The SIGMI does include peristaltic movements, but only those of the stomach. In contrast, the TIM-2 mimics peristaltic movements of the large intestine, as an alternative to mixing by stirrers as in the SHIME. Stirrers can be unfavourable for certain food products, as these can mechanically break certain cell wall structures, thereby influencing food accessibility for the microbiota ${ }^{116,117}$. Moreover, TIM-2 includes a dialysis component. The disadvantage however is that usually there is only a proximal colon mimicked and experiments are performed for only a few days ${ }^{114}$. 


\section{Aims and order of research chapters}

Previous research on the relationship between diet, gut immunity and microbiota has mainly focussed on SCFAs as microbial metabolites, while other metabolites that are likely to be important for human health have received little attention. The role of microbiota variation among individuals in the production of metabolites is also poorly understood. Lastly, the modulating effect of a food matrix compared to isolated compounds is often not taken into account. Therefore, the aim of this thesis was to study the effects of different foods or food components on immune modulation, mainly focussing on activation of the Aryl hydrocarbon Receptor (AhR), and the role of human digestion and metabolism by the microbiota (Figure 6).

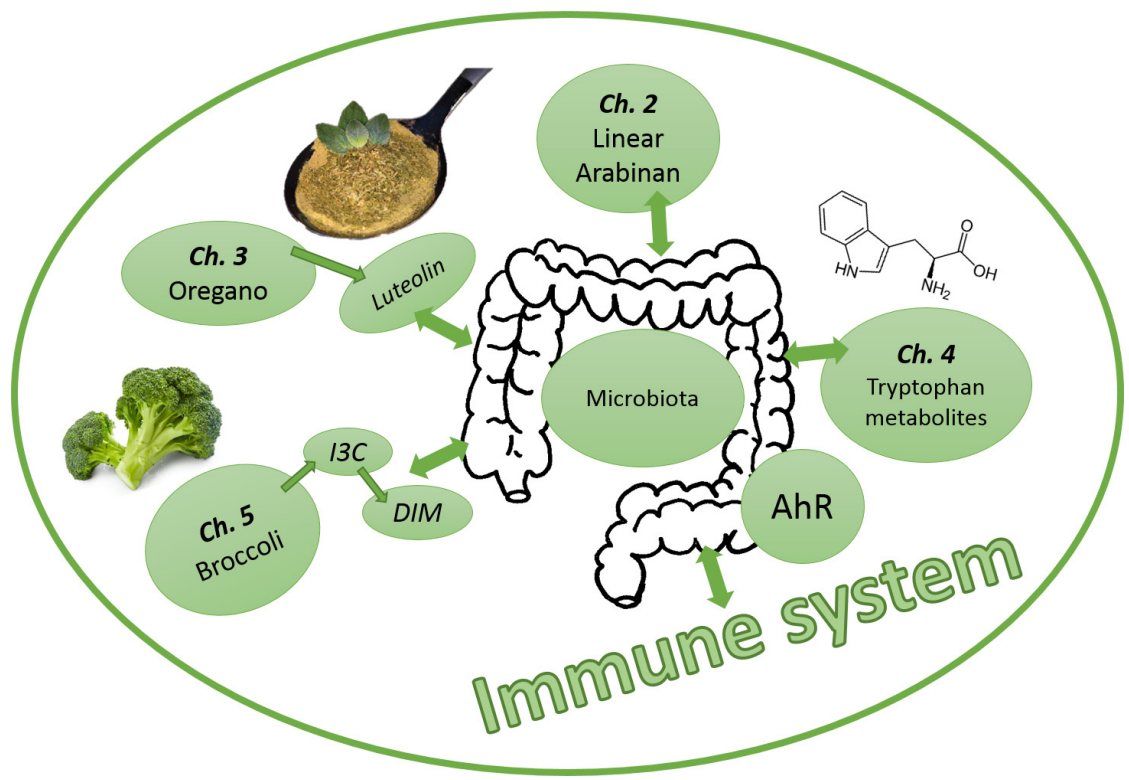

Figure 6. Visual representation of the thesis.

Chapter 2 describes the ability of the dietary fibre arabinan to bind human dendritic cells, and studies the effects of a (food) matrix sugar beet and apple during fermentation of arabinan. In Chapter 3, the effect of oregano, containing the polyphenol luteolin, on AhR activation during in vitro fermentation was studied. As a continuation, Chapter 4 describes the importance of microbial metabolites from tryptophan on AhR activation during in vitro fermentation.

In Chapter 5, we investigated the effects of cooking on the health compounds in broccoli, glucosinolates, and the differences in AhR activation during digestion, both in vitro and in vivo. Finally, Chapter $\mathbf{6}$ discusses the results from the different research chapters, as well as some limitations in the methods used and describes future research opportunities. 


\section{References}

1 Tilg, H. \& Moschen, A. R. Food, Immunity, and the Microbiome. Gastroenterology 148, 1107-1119, (2015).

2 Maslowski, K. M. \& Mackay, C. R. Diet, gut microbiota and immune responses. Nature Immunology 12 5-9, (2011).

3 Van Treuren, W. \& Dodd, D. Microbial Contribution to the Human Metabolome: Implications for Health and Disease. Annual Review of Pathology, (2019).

4 Sanchez-Tapia, M., Tovar, A. R. \& Torres, N. Diet as Regulator of Gut Microbiota and its Role in Health and Disease. Archives of Medical Research 50, 259-268, (2019).

5 Forgie, A. J., Fouhse, J. M. \& Willing, B. P. Diet-Microbe-Host Interactions That Affect Gut Mucosal Integrity and Infection Resistance. Frontiers in Immunology 10, (2019).

6 Koenig, J. E. et al. Succession of microbial consortia in the developing infant gut microbiome. Proceedings of the National Academy of Sciences 108, 4578-4585, (2011).

7 D'Souza, S. et al. Dietary patterns and risk for Crohn's disease in children. Inflammatory Bowel Diseases 14, 367-373, (2007)

8 Hou, J. K., Abraham, B. \& El-Serag, H. Dietary intake and risk of developing inflammatory bowel disease: a systematic review of the literature. The American Journal of Gastroenterology 106, 563, (2011).

9 Thorburn, A. N. et al. Evidence that asthma is a developmental origin disease influenced by maternal diet and bacterial metabolites. Nature Communications 6, 7320, (2015).

10 Mariño, E.et al. Gut microbial metabolites limit the frequency of autoimmune T cells and protect against type 1 diabetes. Nature Immunology 18, 552, (2017).

11 Smith, P. M. et al. The microbial metabolites, short-chain fatty acids, regulate colonic Treg cell homeostasis. Science $\mathbf{3 4 1}$, 569-573, (2013).

12 Zelante, T. et al. Tryptophan Catabolites from Microbiota Engage Aryl Hydrocarbon Receptor and Balance Mucosal Reactivity via Interleukin-22. Immunity 39, 372-385, (2013).

13 Ercolini, D. \& Fogliano, V. Food design to feed the human gut microbiota. Journal of Agricultural and Food Chemistry 66, 3754-3758, (2018)

14 Okumura, R. \& Takeda, K. Maintenance of gut homeostasis by the mucosal immune system. Proceedings of the Japan Academy. Series B, Physical and Biological Sciences 92, 423-435, (2016).

15 Hachimura, S., Totsuka, M. \& Hosono, A. Immunomodulation by food: impact on gut immunity and immune cell function. Bioscience, Biotechnology, and Biochemistry 82, 584-599, (2018).

$16 \mathrm{Li}$, Y. et al. Exogenous stimuli maintain intraepithelial lymphocytes via aryl hydrocarbon receptor activation. Cell 147, 629-640, (2011).

17 Olsan, E. E. et al. Colonization resistance: The deconvolution of a complex trait. Journal of Biological Chemistry 292, 8577-8581, (2017).

18 Trowell, H., Burkitt, D. \& Heaton, K. Definitions of dietary fibre and fibre-depleted foods. Dietary fibre, Fibre-depleted foods and Disease. Academic Press, New York, 21-30, (1985).

19 Dhingra, D., Michael, M., Rajput, H. \& Patil, R. Dietary fibre in foods: a review. Journal of Food Science and Technology 49, 255-266, (2012).

20 Tan, J. et al. Dietary fiber and bacterial SCFA enhance oral tolerance and protect against food allergy through diverse cellular pathways. Cell Reports 15, 2809-2824, (2016).

21 Atarashi, K. et al. Induction of colonic regulatory T cells by indigenous Clostridium species. Science 331 , 337-341, (2011) 
22 Chambers, E. S., Preston, T., Frost, G. \& Morrison, D. J. Role of gut Microbiota-Generated short-chain fatty acids in metabolic and cardiovascular health. Current Nutrition Reports 7, 198-206, (2018).

23 Tan, T. B. et al. Physicochemical, morphological and cellular uptake properties of lutein nanodispersions prepared by using surfactants with different stabilizing mechanisms. Food \& Function 7, 2043-2051, (2016).

24 Scott, K. P., Duncan, S. H. \& Flint, H. J. Dietary fibre and the gut microbiota. Nutrition Bulletin 33, 201211, (2008).

25 Vigsnaes, L. K., Holck, J., Meyer, A. S. \& Licht, T. R. In vitro fermentation of sugar beet arabinooligosaccharides by fecal microbiota obtained from patients with ulcerative colitis to selectively stimulate the growth of Bifidobacterium spp. and Lactobacillus spp. Applied and Environmental Microbiology 77, 8336-8344, (2011).

26 Al-Sheraji, S. H. et al. Prebiotics as functional foods: A review. Journal of Functional Foods 5, 1542-1553, (2013).

27 Meijerink, M. et al. Structure dependent-immunomodulation by sugar beet arabinans via a SYK tyrosine kinase-dependent signalling pathway. Frontiers in Immunology 9, 1972, (2018).

28 Brown, G. D. Innate antifungal immunity: the key role of phagocytes. Annual Review of Immunology 29, 1-21, (2011).

29 Kimberg, M. \& Brown, G. D. Dectin-1 and its role in antifungal immunity. Medical Mycology 46, 631-636, (2008).

30 Plato, A., Willment, J. A. \& Brown, G. D. C-type lectin-like receptors of the dectin-1 cluster: ligands and signaling pathways. International Reviews of Immunology 32, 134-156, (2013).

31 Rösch, C., Gruppen, H. P. D. \& Schols, H. A. P. D. In vitro fermentation and immunomodulating characteristics of dietary fibres, PhD dissertation, Wageningen University, (2016).

32 Denison, M. S., Pandini, A., Nagy, S. R., Baldwin, E. P. \& Bonati, L. Ligand binding and activation of the Ah receptor. Chemico-Biological Interactions 141, 3-24, (2002).

33 Lee, J. S. et al. AHR drives the development of gut ILC22 cells and postnatal lymphoid tissues via pathways dependent on and independent of Notch. Nature Immunology 13, 144, (2012).

34 Gutiérrez-Vázquez, C. \& Quintana, F. J. Regulation of the immune response by the aryl hydrocarbon receptor. Immunity 48, 19-33, (2018).

35 Denison, M. S. \& Nagy, S. R. Activation of the aryl hydrocarbon receptor by structurally diverse exogenous and endogenous chemicals. Annual Review of Pharmacology and Toxicology 43, 309-334, (2003).

36 Kewley, R. J., Whitelaw, M. L. \& Chapman-Smith, A. The mammalian basic helix-loop-helix/PAS family of transcriptional regulators. The International Journal of Biochemistry \& Cell Biology 36, 189-204, (2004).

37 Mimura, J. \& Fujii-Kuriyama, Y. Functional role of AhR in the expression of toxic effects by TCDD. Biochimica et Biophysica Acta (BBA)-General Subjects 1619, 263-268, (2003).

38 Ramadoss, P. \& Perdew, G. H. The transactivation domain of the Ah receptor is a key determinant of cellular localization and ligand-independent nucleocytoplasmic shuttling properties. Biochemistry 44 , 11148-11159, (2005).

39 Lamas, B., Natividad, J. M. \& Sokol, H. Aryl hydrocarbon receptor and intestinal immunity. Mucosal Immunology 11, 1024-1038, (2018).

40 Chen, H.-S. \& Perdew, G. H. Subunit composition of the heteromeric cytosolic aryl hydrocarbon receptor complex. Journal of Biological Chemistry 269, 27554-27558, (1994).

41 Meyer, B. K., Pray-Grant, M. G., Heuvel, J. P. V. \& Perdew, G. H. Hepatitis B virus X-associated protein 2 is a subunit of the unliganded aryl hydrocarbon receptor core complex and exhibits transcriptional enhancer activity. Molecular and Cellular Biology 18, 978-988, (1998). 
42 Shetty, P. V., Bhagwat, B. Y. \& Chan, W. K. p23 enhances the formation of the aryl hydrocarbon receptorDNA complex. Biochemical Pharmacology 65, 941-948, (2003).

$43 \mathrm{Ma}, \mathrm{Q}$. \& Whitlock, J. P. A novel cytoplasmic protein that interacts with the Ah receptor, contains tetratricopeptide repeat motifs, and augments the transcriptional response to 2, 3, 7 , 8-tetrachlorodibenzo-p-dioxin. Journal of Biological Chemistry 272, 8878-8884, (1997).

44 Pongratz, I., Mason, G. \& Poellinger, L. Dual roles of the 90-kDa heat shock protein hsp90 in modulating functional activities of the dioxin receptor. Evidence that the dioxin receptor functionally belongs to a subclass of nuclear receptors which require hsp90 both for ligand binding activity and repression of intrinsic DNA binding activity. Journal of Biological Chemistry 267, 13728-13734, (1992).

45 Kazlauskas, A., Poellinger, L. \& Pongratz, I. Evidence that the co-chaperone p23 regulates ligand responsiveness of the dioxin (Aryl hydrocarbon) receptor. Journal of Biological Chemistry 274, 1351913524, (1999).

46 Jin, U.-H. et al. Short Chain Fatty Acids Enhance Aryl Hydrocarbon (Ah) Responsiveness in Mouse Colonocytes and Caco-2 Human Colon Cancer Cells. Scientific Reports 7, 10163, (2017).

47 Denison, M. S., Fisher, J. M. \& Whitlock, J. P. Inducible, receptor-dependent protein-DNA interactions at a dioxin-responsive transcriptional enhancer. Proceedings of the National Academy of Sciences $\mathbf{8 5}$ 2528-2532, (1988).

48 Probst, M. R., Reisz-Porszasz, S., Agbunag, R. V., Ong, M. S. \& Hankinson, O. Role of the aryl hydrocarbon receptor nuclear translocator protein in aryl hydrocarbon (dioxin) receptor action. Molecular Pharmacology 44, 511-518, (1993).

49 Whitlock Jr, J. P. Mechanistic aspects of dioxin action. Chemical Research in Toxicology 6, 754-763, (1993).

50 Esser, C. The immune phenotype of AhR null mouse mutants: Not a simple mirror of xenobiotic receptor over-activation. Biochemical Pharmacology 77, 597-607, (2009).

51 Hankinson, O. The aryl hydrocarbon receptor complex. Annual Review of Pharmacology and Toxicology 35, 307-340, (1995).

52 Ashida, H. Suppressive effects of flavonoids on dioxin toxicity. BioFactors 12, 201-206, (2000).

53 Ashida, H., Fukuda, I., Yamashita, T. \& Kanazawa, K. Flavones and flavonols at dietary levels inhibit a transformation of aryl hydrocarbon receptor induced by dioxin. FEBS Letters 476, 213-217, (2000).

54 Canivenc-Lavier, M.-C. et al. Comparative effects of flavonoids and model inducers on drug-metabolizing enzymes in rat liver. Toxicology 114, 19-27, (1996).

55 Ciolino, H. P., Daschner, P. J., Wang, T. T. \& Yeh, G. C. Effect of curcumin on the aryl hydrocarbon receptor and cytochrome P450 1A1 in MCF-7 human breast carcinoma cells. Biochemical Pharmacology 56 197-206, (1998).

56 Ciolino, H. P., Daschner, P. J. \& Yeh, G. C. Dietary flavonols quercetin and kaempferol are ligands of the aryl hydrocarbon receptor that affect CYP1A1 transcription differentially. Biochemical Journal 340, 715-722, (1999).

57 Ciolino, H. P., Wang, T. T. \& Yeh, G. C. Diosmin and diosmetin are agonists of the aryl hydrocarbon receptor that differentially affect cytochrome P450 1A1 activity. Cancer Research 58, 2754-2760, (1998).

58 Fukuda, I., Mukai, R., Kawase, M., Yoshida, K.-i. \& Ashida, H. Interaction between the aryl hydrocarbon receptor and its antagonists, flavonoids. Biochemical and Biophysical Research Communications 359 822-827, (2007).

59 Amakura, Y., Tsutsumi, T., Sasaki, K., Yoshida, T. \& Maitani, T. Screening of the inhibitory effect of vegetable constituents on the aryl hydrocarbon receptor-mediated activity induced by 2, 3, 7, 8-tetrachlorodibenzo-p-dioxin. Biological and Pharmaceutical Bulletin 26, 1754-1760, (2003). 
60 Nishiumi, S. et al. Antagonistic and agonistic effects of indigoids on the transformation of an aryl hydrocarbon receptor. Archives of Biochemistry and Biophysics 470, 187-199, (2008).

61 Nishiumi, S., Yoshida, K.-i. \& Ashida, H. Curcumin suppresses the transformation of an aryl hydrocarbon receptor through its phosphorylation. Archives of Biochemistry and Biophysics 466, 267-273, (2007).

62 Amakura, Y. et al. Influence of food polyphenols on aryl hydrocarbon receptor-signaling pathway estimated by in vitro bioassay. Phytochemistry 69, 3117-3130, (2008).

63 Moura-Alves, P. et al. AhR sensing of bacterial pigments regulates antibacterial defence. Nature 512, 387, (2014).

64 Aarts, J. M. et al. Species-specific antagonism of Ah receptor action by 2, 2', 5, 5'-tetrachloro-and 2, 2', 3, 3', 4, 4'-hexachlorobiphenyl. European Journal of Pharmacology: Environmental Toxicology and Pharmacology 293, 463-474, (1995).

65 Vitaglione, P., Morisco, F., Caporaso, N. \& Fogliano, V. Dietary antioxidant compounds and liver health. Critical Reviews in Food Science and Nutrition 44, 575-586, (2005).

66 Khan, M. K. \& Dangles, O. A comprehensive review on flavanones, the major citrus polyphenols. Journal of Food Composition and Analysis 33, 85-104, (2014).

67 Cardona, F., Andrés-Lacueva, C., Tulipani, S., Tinahones, F. J. \& Queipo-Ortuño, M. I. Benefits of polyphenols on gut microbiota and implications in human health. The Journal of Nutritional Biochemistry 24, 1415-1422, (2013).

68 Manach, C., Scalbert, A., Morand, C., Rémésy, C. \& Jiménez, L. Polyphenols: food sources and bioavailability. The American Journal of Clinical Nutrition 79, 727-747, (2004).

69 Fukuda, I. et al. Pigments in green tea leaves (Camellia sinensis) suppress transformation of the aryl hydrocarbon receptor induced by dioxin. Journal of Agricultural and Food Chemistry 52, 2499-2506, (2004).

70 Bonnesen, C., Eggleston, I. M. \& Hayes, J. D. Dietary Indoles and Isothiocyanates That Are Generated from Cruciferous Vegetables Can Both Stimulate Apoptosis and Confer Protection against DNA Damage in Human Colon Cell Lines. Cancer Research 61, 6120-6130, (2001).

71 Busbee, P. B., Nagarkatti, M. \& Nagarkatti, P. S. Natural indoles, indole-3-carbinol (I3C) and 3,3'-diindolylmethane (DIM), attenuate staphylococcal enterotoxin B-mediated liver injury by downregulating miR-31 expression and promoting caspase-2-mediated apoptosis. PLoS One 10, e0118506, (2015).

72 Jin, U. H. et al. Structure-Dependent Modulation of Aryl Hydrocarbon Receptor-Mediated Activities by Flavones. Toxicological Sciences, (2018).

73 Haslam, E. Practical polyphenolics: from structure to molecular recognition and physiological action. (Cambridge University Press, 1998).

74 Cook, N. C. \& Samman, S. Flavonoids-chemistry, metabolism, cardioprotective effects, and dietary sources. The Journal of Nutritional Biochemistry 7, 66-76, (1996).

75 Yao, L. H. et al. Flavonoids in food and their health benefits. Plant foods for human nutrition 59, 113-122, (2004).

76 Clifford, M. N. Anthocyanins-nature, occurrence and dietary burden. Journal of the Science of Food and Agriculture 80, 1063-1072, (2000).

77 Kapusta-Duch, J., Kopec, A., Piatkowska, E., Borczak, B. \& Leszczynska, T. The beneficial effects of Brassica vegetables on human health. Roczniki Państwowego Zakładu Higieny 63, (2012).

78 Capuano, E., Dekker, M., Verkerk, R. \& Oliviero, T. Food as pharma? The case of glucosinolates. Current Pharmaceutical Design 23, 2697-2721, (2017). 
79 Hubbard, T. D. et al. Dietary broccoli impacts microbial community structure and attenuates chemically induced colitis in mice in an Ah receptor dependent manner. Journal of Functional Foods 37, 685-698, (2017).

80 Chen, I., McDougal, A., Wang, F. \& Safe, S. Aryl hydrocarbon receptor-mediated antiestrogenic and antitumorigenic activity of diindolylmethane. Carcinogenesis 19, 1631-1639, (1998).

81 Verhoeven, D. T., Verhagen, H., Goldbohm, R. A., van den Brandt, P. A. \& van Poppel, G. A review of mechanisms underlying anticarcinogenicity by brassica vegetables. Chemico-biological interactions $\mathbf{1 0 3}$ 79-129, (1997).

82 Fahey, J. W., Zalcmann, A. T. \& Talalay, P. The chemical diversity and distribution of glucosinolates and isothiocyanates among plants. Phytochemistry 56, 5-51, (2001).

83 Kissen, R., Rossiter, J. T. \& Bones, A. M. The 'mustard oil bomb': not so easy to assemble?! Localization, expression and distribution of the components of the myrosinase enzyme system. Phytochemistry Reviews 8 69-86 (2009).

84 Aggarwal, B. B. \& Ichikawa, H. Molecular targets and anticancer potential of indole-3-carbinol and its derivatives. Cell Cycle 4, 1201-1215, (2005).

85 Nguyen, L. P. \& Bradfield, C. A. The search for endogenous activators of the aryl hydrocarbon receptor. Chemical Research in Toxicology 21, 102-116, (2007).

86 Bjeldanes, L. F., Kim, J.-Y., Grose, K. R., Bartholomew, J. C. \& Bradfield, C. A. Aromatic hydrocarbon responsiveness-receptor agonists generated from indole-3-carbinol in vitro and in vivo: comparisons with 2, 3, 7, 8-tetrachlorodibenzo-p-dioxin. Proceedings of the National Academy of Sciences 88, $9543-$ 9547, (1991).

87 Jellinck, P. H. et al. Ah receptor binding properties of indole carbinols and induction of hepatic estradiol hydroxylation. Biochemical Pharmacology 45, 1129-1136, (1993).

88 Roager, H. M.\& Licht, T. R. Microbial tryptophan catabolites in health and disease. Nature Communications 9, 3294, (2018)

89 Allegri, G., Costa, C. V., Bertazzo, A., Biasiolo, M. \& Ragazzi, E. Enzyme activities of tryptophan metabolism along the kynurenine pathway in various species of animals. II Farmaco 58, 829-836, (2003).

90 Richard, D. M. et al. L-Tryptophan: Basic Metabolic Functions, Behavioral Research and Therapeutic Indications. International Journal of Tryptophan Research 2, 45-60, (2009).

91 Peters, J. Tryptophan nutrition and metabolism: an overview. Kynurenine and Serotonin pathways 345358 (1991)

92 Oxenkrug, G. F. Genetic and hormonal regulation of tryptophan-kynurenine metabolism. Annals of the New York Academy of Sciences 1122, 35-49, (2007).

93 Fujigaki, S. et al. Species Differences inl-Tryptophan-Kynurenine Pathway Metabolism: Quantification of Anthranilic Acid and Its Related Enzymes. Archives of Biochemistry and Biophysics 358, 329-335, (1998).

94 Gheorghe, C. E. et al. Focus on the essentials: tryptophan metabolism and the microbiome-gut-brain axis. Current Opinion in Pharmacololgy 48, 137-145, (2019).

95 Agus, A., Planchais, J. \& Sokol, H. Gut microbiota regulation of tryptophan metabolism in health and disease. Cell Host \& Microbe 23, 716-724, (2018).

96 Peuhkuri, K., Sihvola, N. \& Korpela, R. Diet promotes sleep duration and quality. Nutrition Research 32 309-319, (2012).

97 Smirnova, A. et al. Evidence for new light-independent pathways for generation of the endogenous aryl hydrocarbon receptor agonist FICZ. Chemical Research in Toxicology 29, 75-86, (2015). 
98 Fritsche, E. et al. Lightening up the UV response by identification of the arylhydrocarbon receptor as a cytoplasmatic target for ultraviolet B radiation. Proceedings of the National Academy of Sciences $\mathbf{1 0 4}$, 8851-8856, (2007).

99 Jönsson, M. E. et al. The tryptophan photoproduct 6-formylindolo [3, 2-b] carbazole (FICZ) binds multiple AHRs and induces multiple CYP1 genes via AHR2 in zebrafish. Chemico-Biological Interactions 181, 447-454, (2009).

100 Lamas, B. et al. CARD9 impacts colitis by altering gut microbiota metabolism of tryptophan into aryl hydrocarbon receptor ligands. Nature Medicine 22, 598-605, (2016).

101 Wikoff, W. R. et al. Metabolomics analysis reveals large effects of gut microflora on mammalian blood metabolites. Proceedings of the National Academy of Sciences 106, 3698-3703, (2009).

102 Dai, Z.-L., Wu, G. \& Zhu, W.-Y. Amino acid metabolism in intestinal bacteria: links between gut ecology and host health. Frontiers in Bioscience 16, 1768-1786, (2011).

103 Hubbard, T. D., Murray, I. A. \& Perdew, G. H. Indole and Tryptophan Metabolism: Endogenous and Dietary Routes to Ah Receptor Activation. Drug Metabolism and Disposition 43, 1522-1535, (2015).

104 Riggio, O. et al. Peripheral and splanchnic indole and oxindole levels in cirrhotic patients: a study on the pathophysiology of hepatic encephalopathy. The American Journal of Gastroenterology 105, 1374, (2010).

105 Hubbard, T. D. et al. Adaptation of the human aryl hydrocarbon receptor to sense microbiota-derived indoles. Scientific Reports 5, 12689, (2015).

106 Li, M. et al. Symbiotic gut microbes modulate human metabolic phenotypes. Proceedings of the National Academy of Sciences 105, 2117-2122, (2008).

107 Selma, M. V., Espin, J. C. \& Tomas-Barberan, F. A. Interaction between phenolics and gut microbiota: role in human health. Journal of Agricultural and Food Chemistry 57, 6485-6501, (2009).

108 Takamura, T.et al. Lactobacillus bulgaricus OLL1181 activates the aryl hydrocarbon receptor pathway and inhibits colitis. Immunology and Cell Biology 89, 817-822, (2011).

109 Murray, I. A., Nichols, R. G., Zhang, L., Patterson, A. D. \& Perdew, G. H. Expression of the aryl hydrocarbon receptor contributes to the establishment of intestinal microbial community structure in mice. Scientific Reports 6, 33969, (2016).

110 Leavy, O. The 'AHR diet' for mucosal homeostasis. Nature Reviews Immunology 11, 806-806, (2011).

111 Nguyen, T. L. A., Vieira-Silva, S., Liston, A. \& Raes, J. How informative is the mouse for human gut microbiota research? Disease Models \& Mechanisms 8, 1-16, (2015).

112 Fois, C. A. M. et al. Models of the Gut for Analyzing the Impact of Food and Drugs. Advanced Healthcare Materials, (2019).

113 Payne, A. N., Zihler, A., Chassard, C. \& Lacroix, C. Advances and perspectives in in vitro human gut fermentation modeling. Trends in Biotechnology 30, 17-25, (2012).

114 Venema, K. \& van den Abbeele, P. Experimental models of the gut microbiome. Best Practice \& Research Clinical Gastroenterology 27, 115-126, (2013).

115 Van de Wiele, T., Van den Abbeele, P., Ossieur, W., Possemiers, S. \& Marzorati, M. in The Impact of Food Bioactives on Health: in vitro and ex vivo models (eds Kitty Verhoeckx et al.) 305-317 (Springer International Publishing, 2015).

116 Dhital, S., Bhattarai, R. R., Gorham, J. \& Gidley, M. J. Intactness of cell wall structure controls the in vitro digestion of starch in legumes. Food \& Function 7, 1367-1379, (2016).

117 Rovalino-Córdova, A. M., Fogliano, V. \& Capuano, E. A closer look to cell structural barriers affecting starch digestibility in beans. Carbohydrate Polymers 181, 994-1002, (2018). 


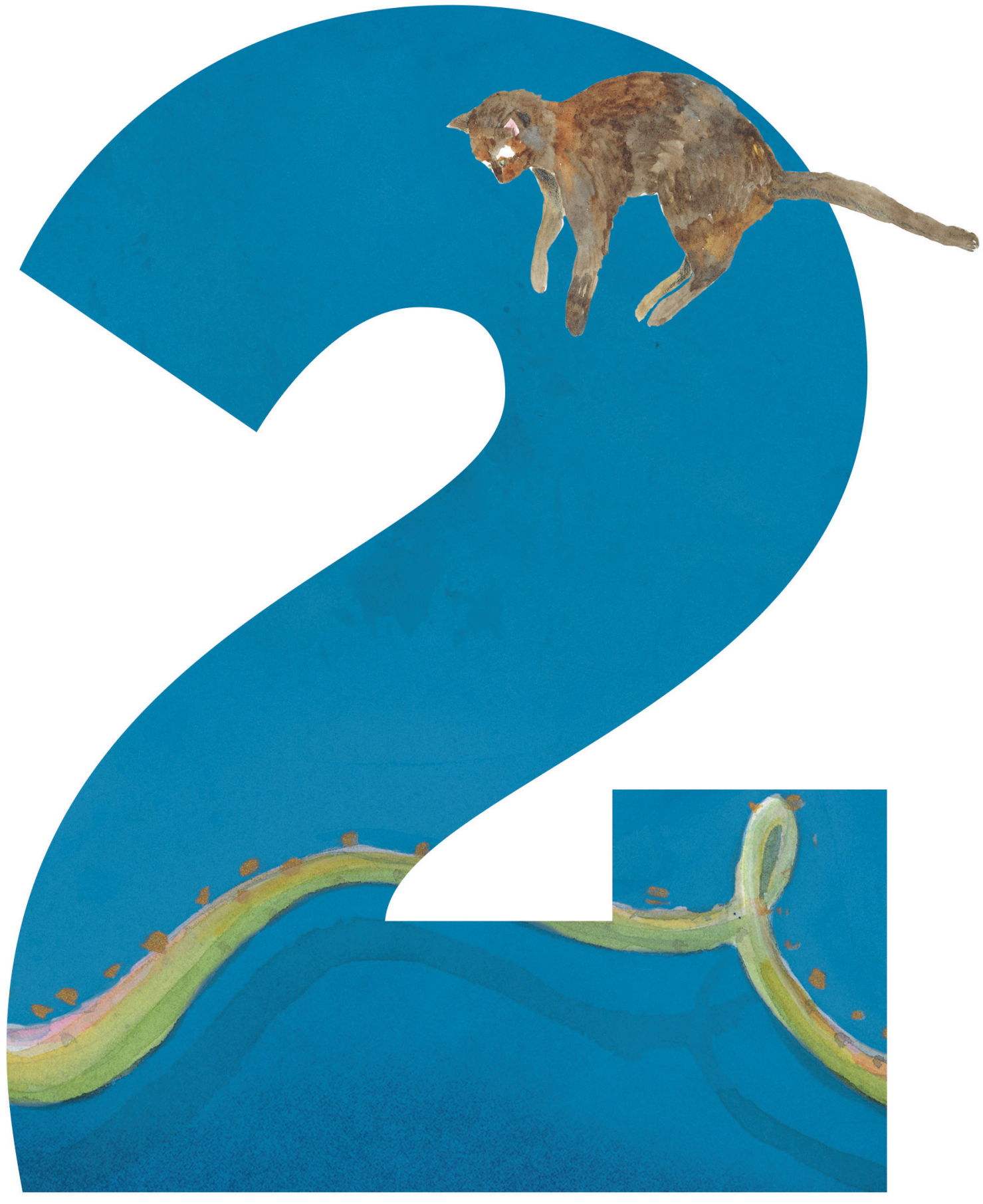




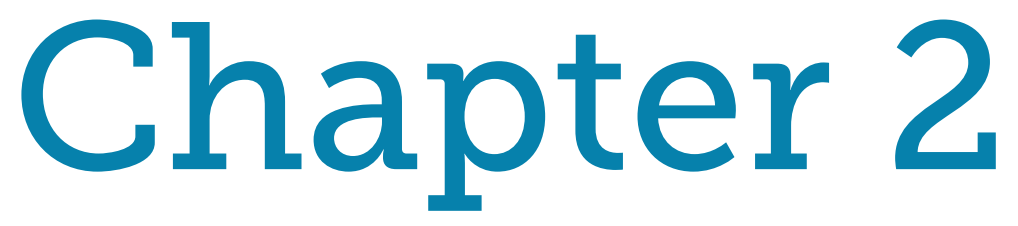

Studies on the in vitro fermentation of pectin and immune-stimulatory mechanism of pectin-derived linear arabinans on human dendritic cells

Jonna EB Koper ${ }^{1,2}$, Joris K Sprokholt ${ }^{3}$, Linda MP Loonen ${ }^{2}$, Edoardo Capuano ${ }^{1}$, Teunis HB Geijtenbeek $^{3}$, Jerry M Wells ${ }^{2}$ 


\section{Abstract}

Dietary fibres such as pectin-derived arabinans are fermented in the colon resulting in bacterial production of short-chain fatty acids (SCFAs), which play a role in gut homeostasis. Additionally, linear arabinan (LA) originating from sugar beet has been reported to strongly activate mouse dendritic cells in a Spleen Tyrosine Kinase (SYK)-dependent manner, suggesting involvement of immune receptor tyrosine-based activating motif (ITAM)-containing C-type lectin receptors (CLR). The aim of this study was to investigate whether LA was immune-stimulatory for human dendritic cells (DCS) and identify the CLR involved. Purified lipopolysaccharide (LPS)-free LA bound specifically to human dendritic cells in a calcium dependent manner with involvement of SYK. Stimulation of human DCs with LA resulted in strong induction of IL-6, IL-10 and TNFa and these responses were reduced after SYK-inhibition. Studies with competitive inhibitors, blocking antibodies and siRNA knockdown ruled out the involvement of Dectin-1, Dectin-2, DCSIGN, Mincle and the Mannose Receptor in immune activation by LA. Additionally, we fermented sugar beet (SB), apple (A) and sugar beet pectin (SBP) with human faecal microbiota in vitro to investigate the potential biological relevance of pectin fermentation and immune activity of the glycans in vivo. Filtered supernatants from SB and A fermentation increased production of IL-6 and TNF $\alpha$ in BMDCs from TLR4/2 knockout mice compared to 'microbiota-only' controls. However, fermentation of isolated SBP only had a small effect on the immune stimulatory activity compared to SB, suggesting an important role for the natural matrix. In conclusion, LA is immune-stimulatory for human DCs in a calcium and SYK-dependent manner, which suggests involvement of a CLR other than the CLRs targeted in this study.

Keywords: Dendritic cells, Immunomodulation, Dietary fibres, Sugar beet, Apple 


\section{Introduction}

Dietary fibres are components of plant cells that are resistant to human digestion ${ }^{1,2}$. They are fermented by the microbiota in the colon to produce short-chain fatty acids (SCFA). The SCFA have important effects on health through various mechanisms ${ }^{3-5}$, ranging from the induction of regulatory Tcells in the colon ${ }^{6-10}$, barrier protection ${ }^{11}$, energy metabolism ${ }^{12-16}$, and entero-endocrine function ${ }^{17,18}$. Dietary fibre fermentation also promotes the growth of specific genera and species of the intestinal microbiota ${ }^{19,20}$, which are associated with health benefits, i.e. they have a prebiotic effect. Apart from their indirect effects on microbiota and SCFA production, several fibres have been reported to have immune-stimulatory effects in immune cell ${ }^{21}$. The mechanisms involved in immunomodulatory effects of fibres are poorly understood with the exception of $\beta$-1,3-linked glucans, which are components of the cell walls of certain fungi. These signal through binding to the C-type lectin receptor (CLR) Dectin-1, which is important for antifungal immunity22-24.

Several CLRs are present on immune cells, such as dendritic cells (DCs) and macrophages, where they play a role in innate immunity and immune regulation ${ }^{25}$. CLRs belong to the superfamily of C-type lectins which contain a common protein fold known as the carbohydrate recognition domain. Several membrane CLRs signal through the immune receptor tyrosine-based activating motif (ITAM) or hemi-ITAM motif signalling domains, which signal through recruitment of spleen tyrosine kinase (SYK). Recruitment of SYK then leads to activation of the CARD9/Bcl10/Malt-1 module to promote NF-KB signalling and expression of inflammatory genes. Dectin-1 is an example of a hemi-ITAM, which requires ligand-induced dimerization of Dectin-1 and bridging the two ITAM domains to recruit SYK ${ }^{26,27}$. Some CLRs possess the immunoreceptor tyrosine-based inhibitory signalling motif (ITIM), which negatively regulates heterologous inflammatory signalling pathways through recruitment of tyrosine phosphatases such as SHP-1 or SHP-2. Several CLRs do not possess ITAM or ITIM motifs in their cytoplasmic tails. One example is DC-SIGN, where ligand binding leads to the formation of a signalling complex containing LSP1, KSR1, and CNK and the kinase Raf-1 that modulates inflammatory signalling through its acetylation of the $\mathrm{p} 65$ subunit of NF-kB.

CLRs can recognise glycosylated self-antigens and microbe-associated glycans, but the specificity and binding mode has only been characterised for a few receptors. Dectin-1 binds to b-1,3 glucans found in fungal cell walls and plays an important role in antifungal immunity22-24. Dectin-2, an ITAM-containing CLR, recognises a-mannan in fungal cell walls, mannose-capped lipoarabinomannan of Mycobacterium tuberculosis and mannosylated O-antigens of Gramnegative bacterial lipopolysaccharides. Mincle, another ITAM containing CLR, is expressed on monocytes, macrophages, dendritic cells and neutrophils. Mincle can bind to a broad range of bacterial pathogens through glycolipids ${ }^{28}$. DC-SIGN is mainly expressed by myeloid and monocyte-derived DCs and binds preferentially to high mannose structures and fucoseterminated glycan structures ${ }^{29}$. 
Several CLRs are expressed in the colon ${ }^{23}$, but, with the exception of Dectin-1, the relevance of other CLR-fibre interactions in the gut are poorly understood. Recently, Dectin-1 was shown to be expressed in primary colonic enterocytes and induce chemokine secretion upon ligand binding to $\beta 1,3$-linked glucans ${ }^{23}$. Binding of dietary fibres to the epithelial associated subsets of macrophages or dendritic cells may also impact intestinal immunity. Evidence that Dectin-1 signalling is indeed important in the gut, comes from the finding that a loss of function polymorphism in Dectin-1 are associated with severe, intractable forms of ulcerative colitis resulting from fungal microorganisms in the intestine.

Recently, bone marrow-derived dendritic cells (BMDCs) from TLR2/4 knockout mice were shown to be a reliable approach to analyse the immunomodulatory properties of a diverse range of dietary fibres, by avoiding immune cell activation due to contaminating MicrobeAssociated Molecular Patterns (MAMPs). Several of the 44 tested dietary fibre preparations induced cytokine responses in BMDCs from TLR2/4 KO mice ${ }^{21}$. Particulate fractions of linear arabinan (LA) and branched arabinan (BA) from sugar beet pectin were shown to be strongly immune-stimulatory with LA being more immune-stimulatory than BA. Moreover, enzymatic debranching of BA increased its immune-stimulatory activity, possibly due to increased particle formation by the alignment of debranched linear arabinan. Mechanistic studies showed that immune stimulation was SYK-dependent but independent of Dectin-1, suggesting recognition of the LA and BA via an unknown C-type lectin receptor ${ }^{21}$.

Here we produced lipopolysaccharide (LPS) and MAMP free LA to investigate whether it was immune-stimulatory for human dendritic cells. To investigate which CLRs might be binding to $L A$, we coupled LA to fluorescent beads and tested binding to human dendritic cells in presence or absence of blocking antibodies and known CLR ligands. Additionally, we tested whether in vitro fermentation by the microbiota of sugar beet (SB), sugar beet pectin (SBP), and apple (A) could generate immune-stimulatory LA when LA was incorporated in a natural matrix. 


\section{Materials and Methods}

\subsection{Substrates}

Linear (1,5)-a-L-Arabinan (LA, originating from sugar beet) was purchased from Megazyme (Ireland). Raw sugar beet was kindly provided by Wageningen farm and Elstar apples were purchased at the local market (Wageningen, The Netherlands).

\subsection{Receptor ligand specificity assays}

A flow cytometry-based bead-binding assay to measure receptor-ligand specificity was used as previously described by Sprokholt et al., 2016 (methods 3.2.3; ${ }^{30}$ ). Fluorescent beads were coated with lyophilised rat monoclonal protein-G affinity purified antibody specific for (1-5)- $a$-LArabinan (LM6, Plantprobes, UK). Lewis Y (LeY) antigen (Lectinity, Russia) was used as positive control for binding.

\subsection{Lipopolysaccharide removal and TLR signalling assays}

In order to assure that linear arabinan was free of any LPS contamination, alkaline treatment was used to remove LPS according to Govers et al., 2016 ${ }^{31}$. Next, HEK293-hTLR2, HEK293hTLR4 and HEK293-hTLR5 cells transfected with pNifty2 vector containing the firefly luciferase gene under control of the NF-kB promoter (Invivogen, France) were used in reporter assays to detect any contamination with LPS or other MAMPs. The HEK293 cell lines were grown in DMEM growth medium with $10 \%$ fetal bovine serum and $1 \%$ penicillin/streptomycin (All Gibco, USA). The cells were plated on opaque clear bottom plates (Corning, USA) at a concentration of $6 \times 10^{4}$ cells per well and grown overnight before 3 hour stimulation with

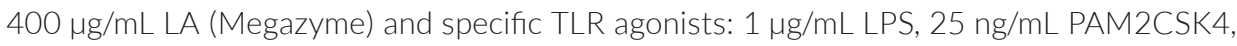
$40 \mathrm{ng} / \mathrm{mL}$ flagellin and $2.5 \mathrm{ng} / \mathrm{mL}$ TNFa (all Invivogen). $120 \mu \mathrm{L}$ cell supernatant was collected and $100 \mu \mathrm{L}$ Bright-Glo substrate (Promega, USA) was added. The plates were shaken for 5 min at 500 rpm after which the luminescence was measured (Spectramax M5, Molecular Devices, USA).

\subsection{Cytokine secretion assays with isolated BMDCs from TLR2/4 KO mice}

Frozen aliquots of BMDC from 3 to 5 months old TLR2/4 knock out female mice were thawed and stimulated with the carbohydrate fractions as previously described ${ }^{21}$. Cells were plated in a concentration of $4 \times 10^{4}$ per well and grown for a week in RPMI growth medium with $10 \%$ fetal calf serum, 1 \% penicillin/streptomycin (all Gibco), 20 ng/mL recombinant mouse granulocyte macrophage colony-stimulating factor (GM-CSF, R\&D systems, USA) and $0.5 \mathrm{LL} / \mathrm{mL} \beta$-mercaptoethanol (Invitrogen, The Netherlands). After a confluent cell layer was formed, cells were stimulated for 24 hours with particulate LA and the following TLR ligands as controls: 10 and 100 ng/mL LPS, 10 ng/mL, Pam2CSK4 (a synthetic diacylated lipopeptide ligand of TLR2 and TLR2/6) and $100 \mathrm{ng} / \mathrm{mL}$ Pam3CSK4 (a synthetic triacylated lipopeptide 
ligand of TLR2 and TLR2/1). As an additional control depleted zymosan, a ligand for Dectin-1, was added at a concentration of $20 \mu \mathrm{g} / \mathrm{mL}$. After $24 \mathrm{~h}$, the cell culture supernatant was collected and cytokines IL-12p70, TNFa, IL-10, IL-6, IL-1 3 and IL-8 were measured using the BD ${ }^{\text {TM }}$ Cytometric Bead Array System (CBA, BD Biosciences, USA), and flow cytometry (Cytoflex Beckman Coulter, USA).

\subsection{Cytokine mRNA expression in human dendritic cells}

Peripheral blood monocytes from healthy donors were isolated as previously described by Sprokholt et al., 201729. Monocytes were differentiated into immature dendritic cells (DCs) by addition of $500 \mathrm{U} / \mathrm{mL}$ IL-4 and $800 \mathrm{U} / \mathrm{mL}$ GM-SCF (Invitrogen) for 1 week in RPMI supplemented with $10 \%$ fetal calf serum, $10 \mathrm{U} / \mathrm{mL}$ penicillin, $10 \mathrm{mg} / \mathrm{mL}$ streptomycin (all Invitrogen) and $2 \mathrm{mM}$ L-glutamine (Lonza, Switzerland). Immature DCs were plated with $1 \times 10^{5}$ cells per well in a U-bottom plate (Corning). Antibodies to Dectin-1, DC-SIGN or a control antibody of the same immunoglobulin isotype were added and incubated for 2 hours, and silencing with siMincle (small inhibitory RNA for macrophage inducible C-type lectin) or siMCL (small inhibitor macrophage C-type lectin) were incubated for 48 hours, after which the stimuli were added and incubated for another 6 hours. Positive controls were used to validate blocking by antibodies as described previously by Gringhuis et al. ${ }^{32,33}$. The cells were lysed and mRNA purified using an mRNA capture kit (Roche, Switzerland). cDNA was synthesized using the reverse transcriptase kit (Promega), followed by RT-PCR in the ABI 7500 Fast PCR detection system (Applied Biosystems). Data analysis was performed with 7500 Fast Dx software.

\subsection{Pectin extraction from sugar beet}

The sugar beet was washed, peeled and sliced into pieces of approximately $4 \times 3 \times 0.3 \mathrm{~cm}\left(\mathrm{~L}^{*} \mathrm{~W}^{*} \mathrm{H}\right)$. Then it was immersed in boiling $96 \%(\mathrm{~V} / \mathrm{V})$ ethanol at $80^{\circ} \mathrm{C}$ for $20 \mathrm{~min}$ and oven-dried at $40^{\circ} \mathrm{C}$ for $24 \mathrm{~h}$. The dried sugar beet was milled using a freeze milling (model 6875D, SPEX Sample Prep, USA) and passed through a $0.25 \mathrm{~mm}$ sieve. To extract the pectin, the powder was mixed with water in a ratio of 1/50 (w/v). The solution was sonicated (Sonation, Germany) for $30 \mathrm{~min}$ after which it was added to $0.5 \mathrm{M} \mathrm{NaOH}$ in a concentration of $40 \mathrm{~mL} / \mathrm{g}$ sugar beet. The mixture was microwaved for $5 \mathrm{~min}$ at $0.9 \mathrm{~kW}$ and cooled in a water bath ${ }^{34,35}$. After cooling, the mixture was centrifuged (ThermoFisher, Germany) at $3494 \mathrm{~g}$ for $20 \mathrm{~min}$. The $\mathrm{pH}$ of the supernatant was adjusted to $\mathrm{pH} 5.5$ with $8 \mathrm{M} \mathrm{HCL}$. Then, the filtrate was coagulated with $96 \%$ ethanol in a volume ratio of 1:1.5 and incubated for 6 hours at room temperature. After incubation, the coagulated pectin was centrifuged at $3494 \mathrm{~g}$ for $10 \mathrm{~min}$ and dispersed in two volumes of $96 \%$ ethanol and dried at $50{ }^{\circ} \mathrm{C}$ in an incubator (BINDER, Germany). The dried sugar beet pectin was milled by Fritsch Mill (FRITSCH, Germany) and stored in a sealed container at room temperature until further experiments. 


\subsection{Sugar composition}

The sugar composition of digested (Section 2.8) sugar beet (SB), digested apple (A), and sugar beet pectin (SBP), was determined by high-performance anion-exchange chromatography (HPAEC), using an ICS-3000 ion chromatography high-performance liquid chromatography (HPLC) system equipped with a CarboPac PA-1 column $(2 \times 250 \mathrm{~mm})$ in combination with a CarboPac PA guard column $(2 \times 25 \mathrm{~mm})$ and a pulsed electrochemical detector in pulsed amperometric detection mode (Dionex, Sunnyvale, USA). First, duplicate samples were hydrolysed in sulphuric acid to generate monomeric sugars according to Seaman et al. ${ }^{36}$, then hydrolysed for $1 \mathrm{~h}$ in $72 \%$ $\mathrm{W} / \mathrm{W} \mathrm{H}_{2} \mathrm{SO}_{4}$ at $30^{\circ} \mathrm{C}$ and finally diluted with distilled water to a final concentration of $1 \mathrm{M} \mathrm{H}_{2} \mathrm{SO}_{4}$. The mixture was incubated for $3 \mathrm{~h}$ at $100^{\circ} \mathrm{C}$, then cooled on ice and then briefly centrifuged. The supernatants were diluted until $1 \mathrm{~mL}$ volume was reached, and $2.5 \mu \mathrm{L} \mathrm{0.1 \%} \mathrm{(w/v)} \mathrm{bromophenol}$ blue in ethanol was added. The $\mathrm{pH}$ was adjusted with barium carbonate until a clear blue colour was obtained $(\mathrm{pH}>4$ 4.6). The remaining solution was filtrated using a $0.45 \mu \mathrm{m}$ PFTE filter. The amount of monomeric sugars was measured according to the method of Gilbert-López et al., with the following modifications ${ }^{37}$. A flow rate of $0.25 \mathrm{~mL} / \mathrm{min}$ was used and the column was equilibrated with $\mathrm{H}_{2} \mathrm{O}$. Elution was performed as follows: 0-35 min $\mathrm{H}_{2} \mathrm{O}$, 35-50 min 0-40 \% 1 $M$ sodium acetate in $100 \mathrm{mM} \mathrm{NaOH}, 50-55 \mathrm{~min} 1 \mathrm{M}$ sodium acetate in $100 \mathrm{mM} \mathrm{NaOH}, 55-60$ min 150 mM NaOH, 70-85 min $\mathrm{H}_{2} \mathrm{O}$. Monomeric sugars eluted from the CarboPac PA guard column were detected after addition of $0.5 \mathrm{M}$ sodium hydroxide $(0.15 \mathrm{~mL} / \mathrm{min})$. The monomeric sugars were quantified using a calibration curve generated with standards in the range of $0-0.1$ $\mathrm{mg} / \mathrm{mL}$. Deoxy-galactose was used as internal standard and the column temperature maintained at $20^{\circ} \mathrm{C}$.

\subsection{In vitro fermentation with human faecal microbiota}

In vitro batch fermentation of fibres by human faecal microbiota was performed as previously described ${ }^{38}$. SB and A were first incubated in $0.9 \%\left(\mathrm{~W} / \mathrm{V}\right.$ ) pancreatin (Sigma) at $37^{\circ} \mathrm{C}$ overnight in order to remove any free sugars. After incubation, the samples were centrifuged and the pellets were freeze-dried and milled into powder. Sterile penicillin bottles were filled with $43 \mathrm{~mL}$ sterilised colon growth medium (5.22 g/L K ${ }_{2} \mathrm{HPO}_{4}, 16.32 \mathrm{~g} / \mathrm{L} \mathrm{KH}_{2} \mathrm{PO}_{4}, 2 \mathrm{~g} / \mathrm{L} \mathrm{NaHCO}, 2 \mathrm{~g} / \mathrm{L}$ yeast extract, $2 \mathrm{~g} / \mathrm{L}$ peptone, $1 \mathrm{~g} / \mathrm{L}$ mucin, $0.5 \mathrm{~g} / \mathrm{L} \mathrm{L}$-cysteine $\mathrm{HCL}$ and $2 \mathrm{~mL} / \mathrm{L}$ tween-80) and with $20 \mathrm{~mL}$ of each substrate in sterilised demi-water: digested sugar beet (0.8 g; SB), digested apple (1.3 g; A) or sugar beet pectin (0.2 g; SBP). The bottles were closed with rubber caps and made anaerobic by flushing with nitrogen. The human faecal inoculums were prepared as described by Van den Abbeele et al..$^{38}$, in a phosphate buffer $\left(8.8 \mathrm{~g} / \mathrm{LK}_{2} \mathrm{HPO}_{4}, 6.8 \mathrm{~g} / \mathrm{LKH}_{2} \mathrm{PO}_{4}\right.$ and $0.1 \mathrm{~g} / \mathrm{L}$ sodium thioglucolate) using separate faecal samples from 2 healthy, non-smoking, donors ageing from 25 - 30 years. To start the fermentation, $7 \mathrm{~mL}$ faecal inoculum was injected into the penicillin bottles. Microbiota-free control bottles were prepared with each substrate, growth medium and phosphate buffer without faecal inoculum. Additionally, a control containing only growth medium and faecal inoculum in phosphate buffer was included for each faecal donor. The 
bottles were incubated at $37^{\circ} \mathrm{C}$ with constant shaking (300 rpm) for $48 \mathrm{~h}$. After O h, $4 \mathrm{~h}, 8 \mathrm{~h}, 24$ h and 48 h, $3 \mathrm{~mL}$ samples were collected, centrifuged at $9000 \mathrm{~g}$ for $5 \mathrm{~min}$, filtered using a $0.2 \mathrm{\mu m}$ RC filter (Phenomenex) and stored at $-20^{\circ} \mathrm{C}$ until further analysis. The supernatants ( $10 \%$ final volume) were incubated with BMDCs from TLR2/4 KO mice for $24 \mathrm{~h}$ and secreted cytokines quantified as described in section 2.4 above. As controls, BMDCs were stimulated with (100 ng/ mL LPS, 10 ng/mL Pam2CSK4, 100 ng/mL Pam3CSK4 or 20 mg/mL depleted zymosan).

\subsection{Statistical analyses}

GraphPad Prism 5 (La Jolla, USA) was used for the statistical analyses. Results are shown as mean \pm SEM. One-way ANOVA followed by a Tukey post-hoc test was used to analyse the data for NF-kB activation in TLR 2, 4 and 5 reporter cells and cytokine measurements. Student's t-test was used to test the difference between bead binding with and without the addition of potential binding inhibitors. Two-way ANOVA was used to analyse qPCR data on the relative expression of cytokines in the presence or absence of piceatannol and blocking antibodies. A repeated measures two-way ANOVA was used for the differences in IL-6 production between the substrates during in vitro fermentation over time, followed by a Bonferroni post-hoc test. For probability values of ${ }^{*} p<0.05,{ }^{* *} p<0.01,{ }^{* * *} p<0.001$ and ${ }^{* * * *} p<0.0001$ the null hypothesis was considered true. 


\section{Results}

Previously, LA was shown to activate BMDCs from mouse TLR2/4 knockout mice which avoided potential effects of contaminants such as bacterial LPS, lipoproteins and lipotechoic acids. In order to investigate the potential immunomodulatory effect of LA (from sugar beet) on human DCs, it was necessary to remove MAMPs such as LPS and TLR2 agonists, which would activate the NF-kB pathway through TLR signalling. The alkali-treated LA gave only background levels of NF-kB activity in TLR luciferase reporter cell lines, which were not significantly different to the medium negative control ( $p<0.05$; Figure 1). The responsiveness of the different TLR-reporter cell lines was verified using their specific ligands and TNFa as a TLR-independent activator of the NF-kB pathway. Control HEK293 cells containing the pNiFTy reporter but not expressing TLRs were, as expected, not responsive to the TLR ligands (data not shown). We also verified that the alkali-treated LA still activated BMDCs from TRL2/4 KO mice and induced cytokine secretion (Figure 2).
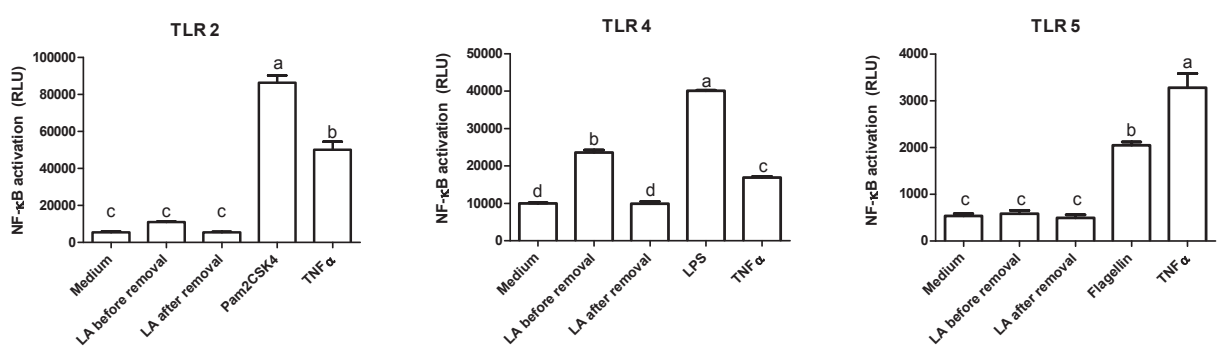

Figure 1. NF-KB activation (RLU) after stimulation of HEK293-hTLR2, HEK293-hTLR4 and HEK293hTLR5 pNifty2 cells with the controls LPS (1 $\mu \mathrm{g} / \mathrm{mL})$, PAM2CSK4 (25 ng/mL), flagellin (40 ng/mL) and TNFa $(2.5 \mathrm{ng} / \mathrm{mL})$, and linear arabinan $(\mathrm{LA}, 400 \mathrm{\mu g} / \mathrm{mL})$ before and after LPS removal. $n=3$. Different letters above bars represent statistically significant differences between responses to each compound.

\subsection{Specific binding of LA to human DCs}

To study if purified LA would bind to human monocyte-derived DCs, we immobilized LA on fluorescent beads coated with protein-G affinity purified antibody specific for (1-5)- $a$-L-Arabinan. The beads coated with LA bound specifically to DCs with approximately $40 \%$ of bead positive cells. This was similar as the binding of LeY-coated beads, which are known to strongly bind to DCs via the DC-SIGN receptor (Figure 3). In contrast, no binding of the uncoated control beads was detected indicating a specific interaction between DCs and the LA glycans. Moreover, binding of LA-coated beads to DCs was significantly decreased in presence of egtazic acid (EGTA), indicating a calciumdependent mode of binding. In agreement with previous studies on calcium-dependence of DCSIGN-ligand interactions ${ }^{39}$, EGTA also decreased binding of the beads coated with LeY to DCs. 

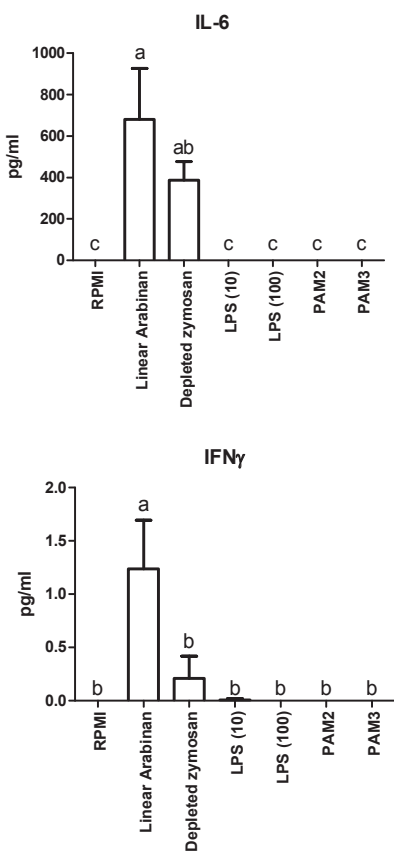

IL-10

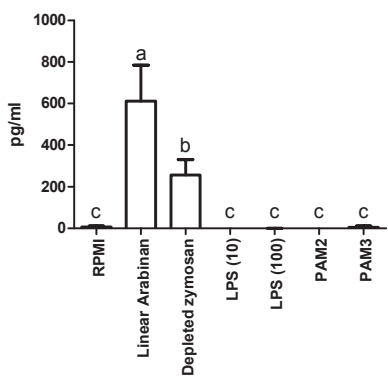

IL-12p70

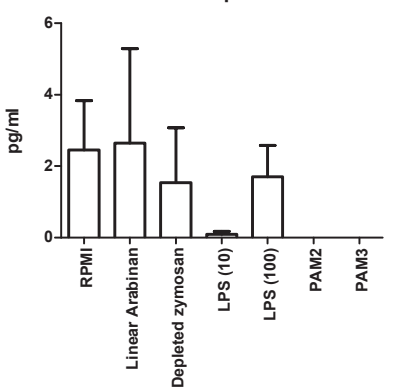

TNF

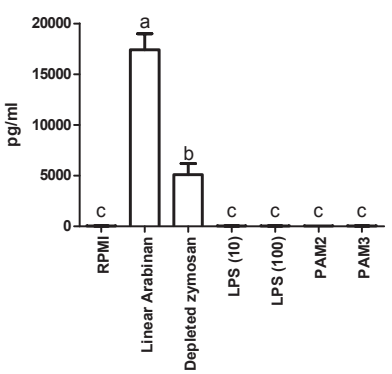

MCP-1

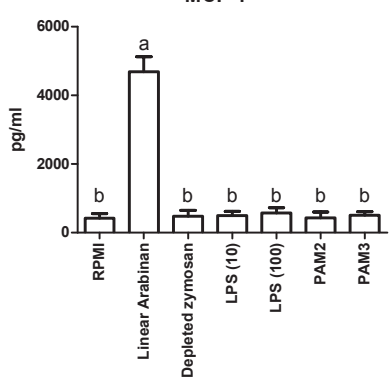

Figure 2. Cytokine production after stimulation of bone marrow derived dendritic cells from TLR2/TLR4 $\mathrm{KO}$ mice with medium control (RPMI), depleted zymosan ( $20 \mathrm{\mu g} / \mathrm{mL}$ ), LPS (10 and $100 \mathrm{ng} / \mathrm{mL}$ ), PAM2 (10 ng/mL), PAM3 (100 ng/mL) or linear arabinan (LA, $400 \mu \mathrm{g} / \mathrm{mL}) . n=3$. Different letters above bars represent statistically significant differences between responses to each compound.

Blocking antibodies against Dectin-1, Dectin-2, combination of Dectin-1 and 2, mannose receptor (MR), or the immunoglobulin isotype control antibody, did not inhibit the binding of LA-coated beads, suggesting that LA binds to a different CLR (Figure 3). Mannan, a competitive inhibitor of mannose-binding receptors, significantly reduced binding of LeY-coated but not LA-coated beads to DCs, indicating that the CLR interacting with LA does not interact with high mannoses (Figure 3).

\subsection{LA activation of human immature DCs}

Addition of purified LPS-free LA to human DCs of two individual donors induced expression of cytokines IL-6, IL-10 and TNFa as shown for BMDCs (Figure 2). Addition of piceatannol (40 $\mu \mathrm{M}$ ) significantly reduced transcription of IL-6 and IL-10 suggesting that the signalling induced by LA may be SYK-dependent (Figure 4a). The cytokine induction by curdlan was effectively blocked by anti-Dectin-1 (positive control, data not shown). The induction of cytokine expression by LA was not blocked by antibodies to Dectin-1 or DC-SIGN compared to a non-specific control antibody of the same immunoglobulin isotype (Figure 4b), providing further evidence that CLRs 
Dectin-1, DC-SIGN or the mannose receptor were not responsible for the signalling and immune activation by LA. Additionally, siRNA silencing of Macrophage (inducible) C-type lectin (MCL and Mincle) did not attenuate expression of cytokines induced by LA (Figure 4c).

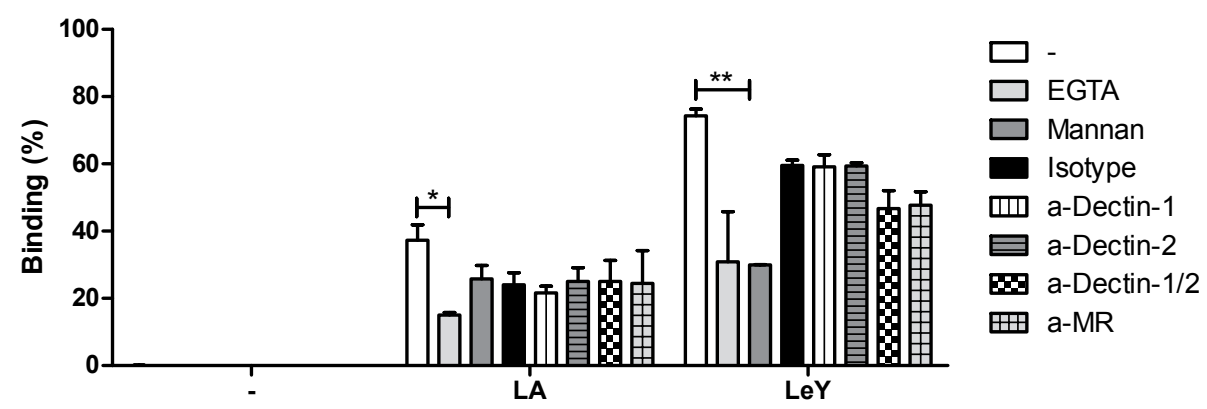

Figure 3. Bead binding assay using beads coated with control (-), linear arabinan (LA) or lewis Y (LeY, positive control) after stimulation of human dendritic cells with EGTA, mannan, isotype, several Dectin antibodies and mannose receptor antibody (a-MR). $n=2 .{ }^{*} p<0.05,{ }^{* *} p<0.01$.

\subsection{Arabinan from faecal microbiota fermentation of sugar beet and apple in vitro}

\subsubsection{Sugar composition of sugar beet, apple and sugar beet derived pectin}

The sugar composition of pancreatin treated SB, A and SBP was presented in Table 1. In all the arabinose substrates, the major sugars present were glucose and arabinose, followed by galactose, galacturonic acid, rhamnose, xylose, mannose and $\mathrm{N}$-acetyl glucosamine. The highest content in galacturonic acid, $12 \% \pm 0.81 \%$ was found in SBP. The arabinose content was $35 \%$ $\pm 0.05 \%$ in SBP which was significant higher $(p<0.05)$ than in SB $(23 \% \pm 0.47 \%)$.

\subsubsection{Immune-stimulatory activity of fermented apple, sugar beet and extracted sugar beet pectin (SBP)}

An in vitro batch model was used to simulate human colon microbiota fermentation of the pancreatic pre-digested SB and A, and extracted SBP. Fermenter supernatants were collected at intervals over $48 \mathrm{~h}$, filtered and added to BMDCs from TLR2/4 KO mice to assess their immune-stimulatory activity by measurement of secreted cytokines (Figure 5). Microbiotafree control bottles were prepared with each substrate, growth medium and phosphate buffer without faecal inoculum. Additionally, a "no-substrate control", containing only growth medium and faecal inoculum in phosphate buffer was included for each faecal donor. 
Table 1. Sugar composition (\% w/w) of the total amount of polysaccharides in sugar beet, apple and sugar beet pectin.

\begin{tabular}{lccc}
\hline & Glucose & Arabinose & Galactose \\
\hline Sugar beet & $50 \pm 0.65$ & $23 \pm 0.47$ & $10 \pm 00.3$ \\
Sugar beet pectin & $36 \pm 0.06$ & $35 \pm 0.05$ & $8 \pm 0.03$ \\
Apple & $50 \pm 0.75$ & $14 \pm 0.15$ & $11 \pm 0.23$ \\
\hline
\end{tabular}
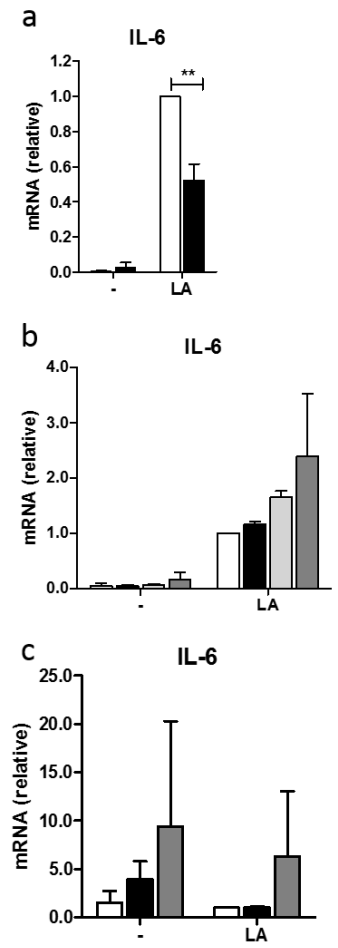
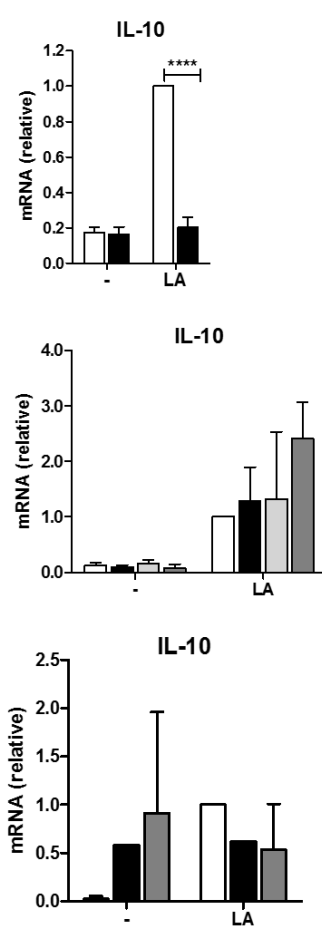
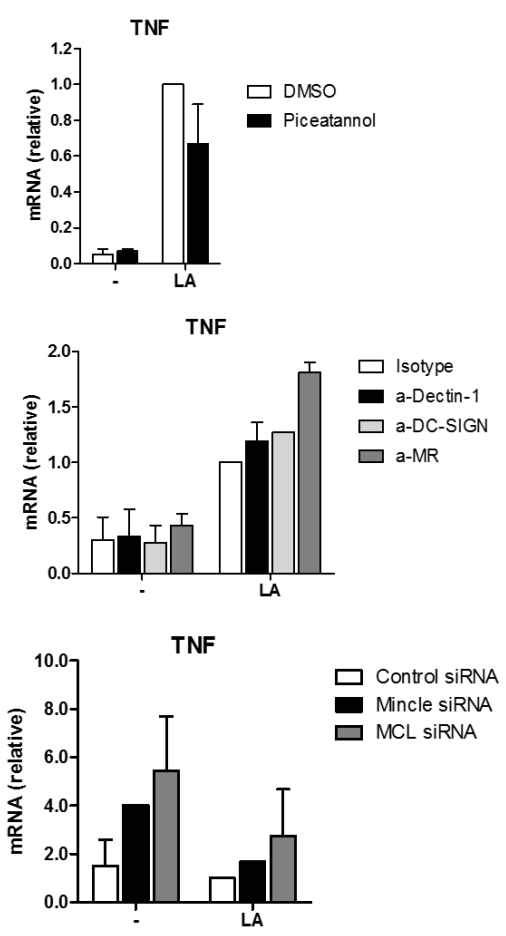

Figure 4. Relative mRNA cytokine levels of IL-6, IL-10 and TNFa after stimulation of human DCs with control (-) or linear arabinan (LA), with the addition of (a) DMSO or Piceatannol, (b) isotype control, antiDectin-1, anti-DC-SIGN or anti-Mannose receptor (a-MR) or (c) silencing of Macrophage (inducible) C-type lectin (MCL and Mincle). $n=2$ donors. ${ }^{* *} p<0.01$, and ${ }^{* * *} p<0.0001$. 


\begin{tabular}{ccccc} 
Galacturonic acid & Rhamnose & Xylose & Mannose & $\begin{array}{c}\text { N-acetyl } \\
\text { glucosamine }\end{array}$ \\
\hline $7 \pm 0.3$ & $2 \pm 0.04$ & $2 \pm 0.03$ & $2 \pm 0.03$ & $3 \pm 0.22$ \\
$12 \pm 0.81$ & $3 \pm 0.12$ & $1 \pm 0.01$ & $1 \pm 0.04$ & $4 \pm 0.81$ \\
$7 \pm 0.02$ & $2 \pm 0.1$ & $7 \pm 0.12$ & $3 \pm 0.01$ & $5 \pm 0.12$ \\
\hline
\end{tabular}
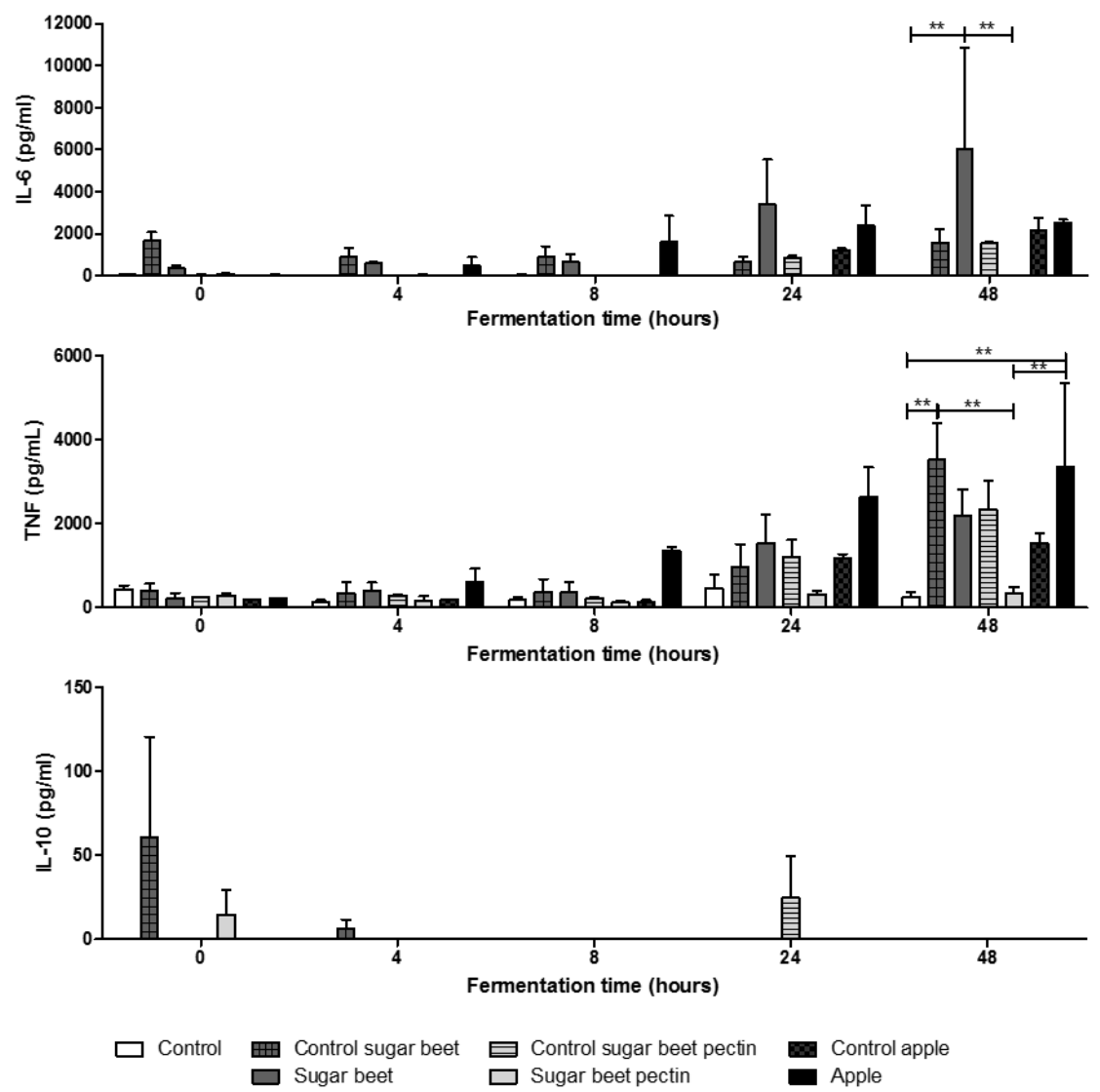

Figure 5. Cytokine production after stimulation of bone marrow derived dendritic cells from TLR2/TLR4 $\mathrm{KO}$ mice with batch fermentation supernatant of nonbacterial control (control), non-bacterial control sugar beet (control sugar beet), sugar beet, non-bacterial control sugar beet pectin (control sugar beet pectin), sugar beet pectin, non-bacterial control apple (control apple) or apple during in vitro fermentation. $n=2$ donors. ${ }^{* *} p<0.001$. 
Fermentation of sugar beet induced a higher IL-6 production by DCs after $48 \mathrm{~h}$ of fermentation than the no-substrate control (SB control, Figure 5; $p<0.001$ ). Besides, after fermentation of SB for $48 \mathrm{~h}$, there was a significant increase in IL-6 compared to SBP fermentation (Figure 5; $p<$ 0.001). TNFa secretion was significantly higher in the microbiota-free SB control compared to the no-substrate control or SBP after 48h ( $p<0.001)$. During A fermentation, there was a significant increase in TNFa production induced by A compared to the SBP and the no-substrate (faecal microbiota only) control. No statistically significant increase in IL-10 production was found for any of the substrates. Furthermore, no differences were observed between a substrate and its corresponding control without bacteria (SB, A or SBP control). 


\section{Discussion}

Immune-modulation by dietary polysaccharides has received increasing interest in recent years $31,40,41$. However, many fibres are contaminated with bacterial MAMPs such as LPS, which are difficult to remove, making it hard to screen for immune activation by human cells ${ }^{31}$. Recently, several dietary fibres were shown to activate BMDCs from TLR2/4 KO mice, that are not responsive to LPS, suggesting that, similar to 1,3- $\beta$-glucan, they interact with CLRs to induce immune signalling responses ${ }^{21}$. Here we verified that LA activates BMDCs from TLR2/4 $\mathrm{KO}$ mice and induces cytokine secretion in amounts comparable to that previously reported (Figure 2). The amounts of secreted cytokines produced after stimulation with $400 \mathrm{\mu g} / \mathrm{mL} \mathrm{LA}$ in our study are comparable to those reported by Meijerink et al. ${ }^{21}$, despite having used frozen aliquots of BMDCs in our study.

To investigate whether LA would also activate human DCs, we used a recently developed method for removing LPS and other MAMPs from dietary fibres ${ }^{31}$. LPS-free LA lacked NF-kB inducing activity in TLR4, 2, and 5 reporter cell assays but still activated BMDCs from TLR4/2 knockout mice. Incubation of LA with human DCs led to activation and cytokine secretion showing that the mechanism of immune activation is also present in human immune cells (Figure 4).

To characterize the human receptor for LA, LPS-free LA was coupled to fluorescent beads with a monoclonal antibody and incubated with human DCs in the presence of different inhibitors, competitors and CLR blocking antibodies. LA binding to human DCs was blocked by the addition of EGTA, indicating that LA binding to DCs is calcium dependent. Ligand binding to the carbohydrate recognition domain of several CLRs is known to be calcium-dependent ${ }^{42}$. Furthermore, DC activation and cytokine secretion was blocked by addition of piceatannol, a SYK inhibitor. The first known SYK coupled C-type lectin was Dectin-1, which functions during fungal infections, but recently other CLRs that signal via SYK have been identified ${ }^{43}$. The finding that LA signals through SYK in a calcium-dependent manner provides evidence for possible involvement of a CLR ${ }^{43,44}$. However, we were unable to identify the CLR recognising LA using CLR blocking antibodies or siRNAs inhibiting the expression of specific CLR. LA bead binding to human DC was not blocked by antibodies against Dectin-1, Dectin-2, a combination of Dectin-1 and Dectin-2, or the mannose receptor, indicating that none of these CLRs interacts with LA. Besides binding, the induction of cytokines was not lowered by antibodies to Dectin-1, Dectin-2, DC-SIGN, a control isotype, and mannose receptor, confirming that LA is recognized by a different C-type lectin receptor. Silencing of Mincle and $\mathrm{MCL}$ also did not decrease the cytokine production by LA, suggesting that LA binding to DCs signals to an unknown other CLR (like) receptor. 
To study the effect of the food matrix and microbiota fermentation on LA release and immune response, pectin-rich matrices such as SB, A and SBP were fermented in vitro by faecal microbiota from two different donors and the supernatants were tested for their capacity to activate cytokine secretion in TLR2/4 KO mice BMDCs. Controls were included where the pectin-rich substrates were dissolved in the growth medium without bacteria to separately check for the effect of soaking and dissolution of potentially active fractions from the substrates. Our hypothesis was that the observed effect of LA to induce cytokine production by DCs would be different when LA is provided in a purified form, when provided still attached to the pectin backbone, and when pectin itself is naturally embedded in the plant cell wall material. The pectin extraction aimed to retain the highest amount of arabinan. As shown in Table 1, the arabinose content in \% w/w was significantly increased in the SBP compared to the SB. This indicates that the pectin extraction was successful and that the SBP probably contains a relatively higher amount of (linear) arabinan.

The major cytokines that were produced by DCs upon stimulation of the supernatants were IL-6 and TNFa. Overall, both SB and A gave a significantly higher cytokine production than the non-substrate bacteria-only control, therefore indicating their potential to induce an immune response. During the fermentation of SB, only a slight increase of IL-6 and TNFa was found over time. Interestingly, the sugar beet control, where no bacterial inoculum was added to the batch fermentation, did not induce a different cytokine production compared to the supernatants from fermented sugar beet. This indicates that the production of cytokines is not, or not prominently, modulated by microbial utilization of sugar beet dietary fibre. It must be stressed here that the BMDCs were stimulated with supernatant of the batch fermentation only, which is expected to contain only the water-soluble fraction of sugar beet and apple to avoid adding intact bacteria which might be phagocytosed. To study the effect of fermentation on the full potential of LA to bind DCs, it would be interesting to study also the insoluble pellet, because the particulate fraction of $L A$ is better able to bind $D C s^{21}$. It is also noteworthy to mention that $S B$ and $A$ have been extensively digested overnight before fermentation, to remove all the possible non-dietary fibre (DF) compounds from the plant matrix. However, it is possible that the comparatively longer incubation for $48 \mathrm{~h}$ at $37^{\circ} \mathrm{C}$ may have facilitated further solubilisation of fibre from the matrix. In such a scenario, the cytokine response observed at different fermentation times would be the result of a complex interplay between release of pectin from the matrix, release of LA molecules from pectin, release of other potentially active compounds from the plant matrix and the metabolism of all by microbial fermentation. After $48 \mathrm{~h}$, SBP induced a significantly lower amount of IL-6 than SB, a trend observed at all-time points, indicating that in SBP LA was probably metabolised during fermentation resulting in a lower IL-6 production. The study of Tuncil et al., showed that fermentation of soluble fibres was faster when fermented individually as compared to when fermented in a mixture ${ }^{45}$. This indicates that SBP was probably fermented faster than the SB, therefore resulting in a rapid loss of $L A$ and less cytokine production by DCS. 
Compared to SB, fermentation of A resulted in less IL-6 production. The observed differences may be due to a difference in the amount of (linear) arabinans in apple pectin, as the amount of substrate supplied in the batch fermentation was calculated based on the arabinose content which might not relate exactly to the amount of LA. However, the difference in the supplied amounts of sugar beet and apple cannot account for the difference in IL-6 production during fermentation. Thus, immunomodulatory fibres such as linear arabinan might be present in greater amounts in A compared to SB. Overall, both SB and apple elicited significantly increased production of cytokines than the non-substrate bacteria-only control, indicating their potential to induce an immune response. 


\section{Concluding remarks}

LA induces cytokine production in human DCs which was not due to contamination with LPS or other MAMPs. Through binding assays with blocking antibodies, competitive ligands as well as siRNA knockdown experiments in human DCs we ruled out the involvement of several well characterised CLRs including Dectin-1/Dectin-2, Mincle, DC-SIGN and the mannose receptor. However, activation of DCs was calcium-dependent supporting the notion that LA interacts with one of the CLRs with a calcium-dependent carbohydrate binding domain. Additionally, activation of DCs and cytokine secretion appeared to be SYK-dependent suggesting the CLR involved contains an ITAM motif or hemi-ITAM motif.

During fermentation of SB and A, mainly IL-6 and TNFa were produced by mice DCs. SBP did not induce higher amounts of cytokine secretion than SB and A after 48 hours of fermentation. This may be due to more rapid microbial metabolism of LA or branched arabinans in isolated pectin.

\section{Acknowledgements}

Research presented in this publication was financially supported by the Graduate School VLAG.

\section{Conflicts of interest}

The authors declare no competing interests. 


\section{References}

1 Trowell, H., Burkitt, D. \& Heaton, K. Definitions of dietary fibre and fibre-depleted foods. Dietary fibre, Fibre-depleted Foods and Disease. Academic Press, New York, 21-30, (1985).

2 Dhingra, D., Michael, M., Rajput, H. \& Patil, R. Dietary fibre in foods: a review. Journal of Food Science and Technology 49, 255-266, (2012).

3 Tan, J. et al. Dietary fiber and bacterial SCFA enhance oral tolerance and protect against food allergy through diverse cellular pathways. Cell Reports 15, 2809-2824, (2016).

4 Atarashi, K. et al. Induction of colonic regulatory T cells by indigenous Clostridium species. Science $\mathbf{3 3 1}$, 337-341, (2011).

5 Chambers, E. S., Preston, T., Frost, G. \& Morrison, D. J. Role of gut Microbiota-Generated short-chain fatty acids in metabolic and cardiovascular health. Current Nutrition Reports 7, 198-206, (2018).

6 Liu, T.et al. Short-chain fatty acids suppress lipopolysaccharide-induced production of nitric oxide and proinflammatory cytokines through inhibition of NF-kappaB pathway in RAW264.7 cells. Inflammation 35, 1676-1684, (2012).

7 Smith, P. M. et al. The microbial metabolites, short-chain fatty acids, regulate colonic Treg cell homeostasis. Science 341, 569-573, (2013).

8 Maslowski, K. M. et al. Regulation of inflammatory responses by gut microbiota and chemoattractant receptor GPR43. Nature 461, 1282-1286, (2009).

9 Atarashi, K. et al. Induction of colonic regulatory T cells by indigenous Clostridium species. Science $\mathbf{3 3 1}$, 337-341, (2011).

10 Atarashi, K. et al. Treg induction by a rationally selected mixture of Clostridia strains from the human microbiota. Nature 500, 232-236, (2013).

11 Kelly, C. J. et al. Crosstalk between microbiota-derived short-chain fatty acids and intestinal epithelial HIF augments tissue barrier function. Cell Host Microbe 17, 662-671, (2015).

12 Canfora, E. E., Jocken, J. W. \& Blaak, E. E. Short-chain fatty acids in control of body weight and insulin sensitivity. Nature Reviews Endocrinology 11, 577-591, (2015).

13 Blouin, J.-M. et al. Butyrate elicits a metabolic switch in human colon cancer cells by targeting the pyruvate dehydrogenase complex. International Journal of Cancer 128, 2591-2601, (2011).

14 Donohoe, Dallas R. et al. The microbiome and butyrate regulate energy metabolism and autophagy in the mammalian colon. Cell Metabolism 13, 517-526, (2011).

15 Chi, L.et al. Manganese-induced sex-specific gut microbiome perturbations in C57BL/6 mice. Toxicology and Applied Pharmacology 331, 142-153, (2017).

16 Collins, H. M. et al. Review: Variability in Fine Structures of Noncellulosic Cell Wall Polysaccharides from Cereal Grains: Potential Importance in Human Health and Nutrition. Cereal Chemistry Journal 87, 272-282, (2010).

17 Tolhurst, G. et al. Short-chain fatty acids stimulate glucagon-like peptide-1 secretion via the G-proteincoupled receptor FFAR2. Diabetes 61, 364-371, (2012).

18 Larraufie, P. et al. SCFAs strongly stimulate PYY production in human enteroendocrine cells. Scientific Reports 8, 74, (2018).

19 Vigsnaes, L. K., Holck, J., Meyer, A. S. \& Licht, T. R. In vitro fermentation of sugar beet arabinooligosaccharides by fecal microbiota obtained from patients with ulcerative colitis to selectively stimulate the growth of Bifidobacterium spp. and Lactobacillus spp. Applied and Environmental Microbiology 77, 8336-8344, (2011). 
20 Al-Sheraji, S. H. et al. Prebiotics as functional foods: A review. Journal of Functional Foods 5, 1542-1553, (2013).

21 Meijerink, M. et al. Structure dependent-immunomodulation by sugar beet arabinans via a SYK tyrosine kinase-dependent signalling pathway. Frontiers in immunology 9, 1972, (2018).

22 Brown, G. D. Innate antifungal immunity: the key role of phagocytes. Annual Review of Immunology 29 , 1-21, (2011).

23 Kimberg, M. \& Brown, G. D. Dectin-1 and its role in antifungal immunity. Medical Mycology 46, 631-636, (2008).

24 Plato, A., Willment, J. A. \& Brown, G. D. C-type lectin-like receptors of the dectin-1 cluster: ligands and signaling pathways. International Reviews of Immunology 32, 134-156, (2013).

25 Johannssen, T. \& Lepenies, B. Glycan-based cell targeting to modulate immune responses. Trends in Biotechnology 35, 334-346, (2017).

26 Kerrigan, A. M. \& Brown, G. D. Syk-coupled C-type lectins in immunity. Trends in Immunology 32, 151 156, (2011).

27 Castro-Bravo, N., Wells, J. M., Margolles, A. \& Ruas-Madiedo, P. Interactions of surface exopolysaccharides from Bifidobacterium and Lactobacillus within the intestinal environment. Frontiers in Microbiology 9, (2018).

28 Wevers, B. A. et al. Fungal engagement of the C-type lectin mincle suppresses dectin-1-induced antifungal immunity. Cell Host \& Microbe 15, 494-505, (2014).

29 Sprokholt, J. K., Heineke, M. H., Kaptein, T. M., van Hamme, J. L. \& Geijtenbeek, T. B. DCs facilitate B cell responses against microbial DNA via DC-SIGN. PloS one 12, e0185580, (2017).

30 Sprokholt, J. K., Hertoghs, N. \& Geijtenbeek, T. B. Flow Cytometry-Based Bead-Binding Assay for Measuring Receptor Ligand Specificity. Methods in Molecular Biology 1390, 121-129, (2016).

31 Govers, C. et al. Lipopolysaccharide quantification and alkali-based inactivation in polysaccharide preparations to enable in vitro immune modulatory studies. Bioactive Carbohydrates and Dietary Fibre 8, 15-25, (2016).

32 Gringhuis, S. I. et al. Dectin-1 directs T helper cell differentiation by controlling noncanonical NF-kB activation through Raf-1 and Syk. Nature Immunology 10, 203-213, (2009).

33 Gringhuis, S. I. et al. Selective C-Rel activation via Malt1 controls anti-fungal TH-17 immunity by dectin-1 and dectin-2. PLoS Pathogens 7, e1001259, (2011).

34 Bagherian, H., Ashtiani, F. Z., Fouladitajar, A. \& Mohtashamy, M. Comparisons between conventional, microwave-and ultrasound-assisted methods for extraction of pectin from grapefruit. Chemical Engineering and Processing: Process Intensification 50, 1237-1243, (2011).

35 Sun, R. \& Hughes, S. Fractional isolation and physico-chemical characterization of alkali-soluble polysaccharides from sugar beet pulp. Carbohydrate Polymers 38, 273-281, (1999).

36 Saeman, J. F., Moore, W. E., Mitchell, R. L. \& Millett, M. A. Techniques for the determination of pulp constituents by quantitiative paper chromatography. Tappi Journal 37, 336-343, (1954).

37 Gilbert-López, B. et al. Downstream processing of Isochrysis galbana: a step towards microalgal biorefinery. Green Chemistry 17, 4599-4609, (2015).

38 Van den Abbeele, P.et al. Arabinoxylo-Oligosaccharides and Inulin Impact Inter-Individual Variation on Microbial Metabolism and Composition, Which Immunomodulates Human Cells. Journal of Agricultural and Food Chemistry 66, 1121-1130, (2018).

39 Geijtenbeek, T. B. et al. DC-SIGN, a dendritic cell-specific HIV-1-binding protein that enhances transinfection of T cells. Cell 100, 587-597, (2000).

40 Schepetkin, I. A. \& Quinn, M. T. Botanical polysaccharides: Macrophage immunomodulation and therapeutic potential. International Immunopharmacology 6, 317-333, (2006). 
41 Wismar, R., Brix, S., Frøkiær, H. \& Lærke, H. N. Dietary fibers as immunoregulatory compounds in health and disease. Annals of the New York Academy of Sciences 1190, 70-85, (2010).

42 Hoving, J. C., Wilson, G. J. \& Brown, G. D. Signalling C-type lectin receptors, microbial recognition and immunity. Cellular Microbiology 16, 185-194, (2014).

43 Kerrigan, A. M. \& Brown, G. D. Syk-coupled C-type lectins in immunity. Trends in Immunology 32, 151156, (2011).

44 Geijtenbeek, T. B. H. \& Gringhuis, S. I. Signalling through C-type lectin receptors: shaping immune

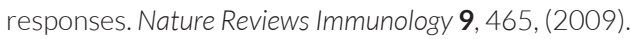

45 Tuncil, Y. E. et al. Delayed utilization of some fast-fermenting soluble dietary fibers by human gut microbiota when presented in a mixture. Journal of Functional Foods 32, 347-357, (2017). 


\section{Supporting information}
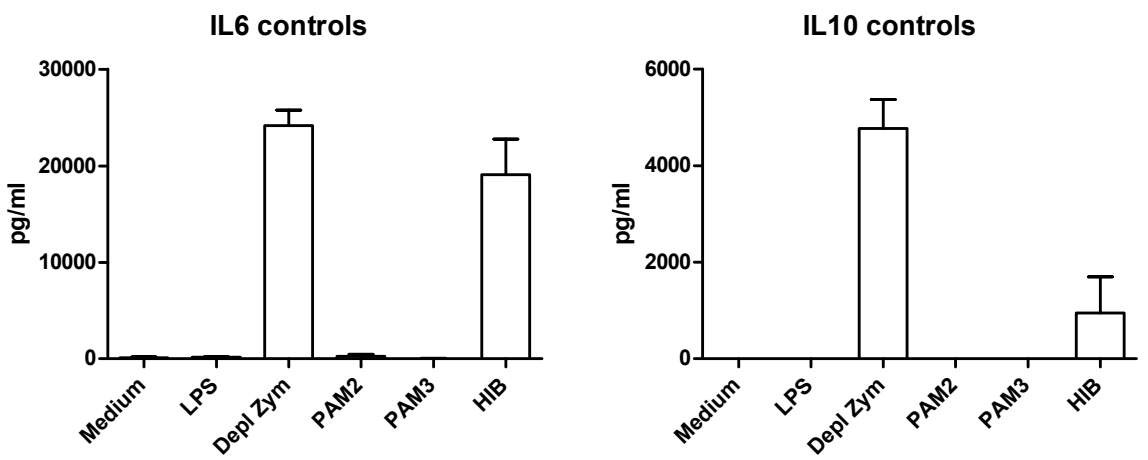

MCP-1 controls

\section{TNF controls}
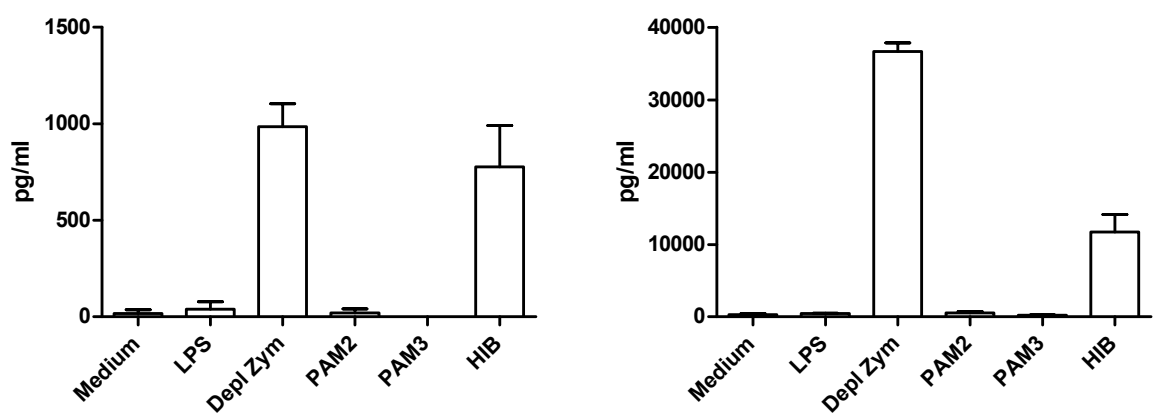

Figure S1. Cytokine production after stimulation of bone marrow derived dendritic cells from TLR2/TLR4 $\mathrm{KO}$ mice with controls: medium, LPS, depleted zymosan, PAM2 or PAM 3. $n=5$. 



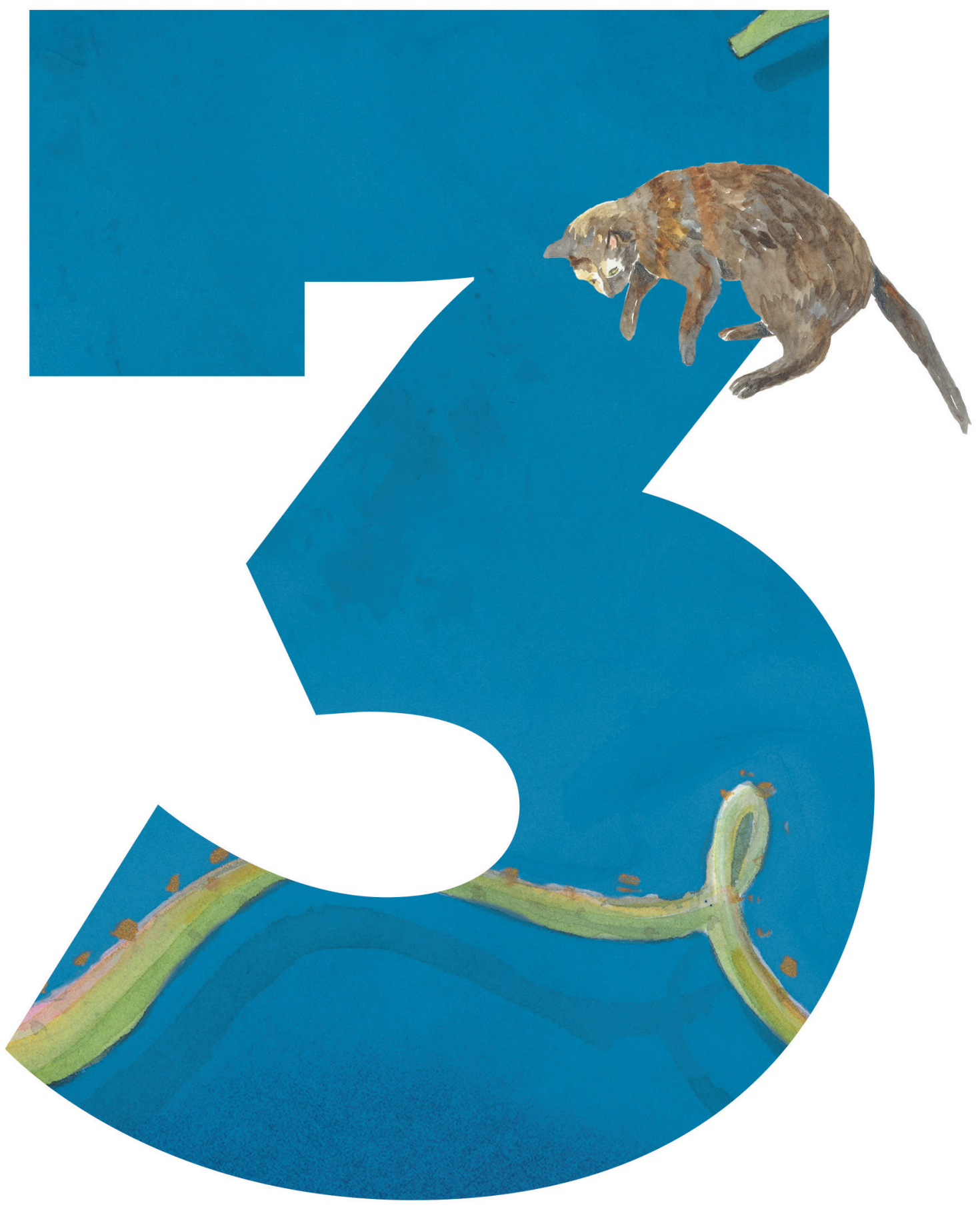




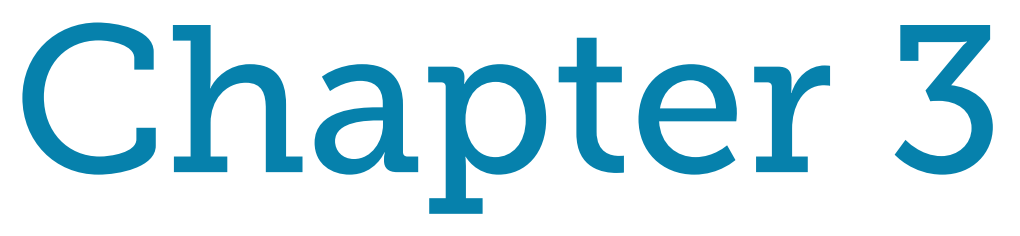

\section{Polyphenols and tryptophan metab- olites activate the Aryl hydrocarbon Receptor in an in vitro model of colonic fermentation}

Jonna EB Koper ${ }^{1,2}$, Linda MP Loonen², Jerry M Wells ${ }^{2}$, Antonio Dario Troise ${ }^{3}$, Edoardo Capuano ${ }^{1}$, Vincenzo Fogliano ${ }^{1}$

\footnotetext{
${ }^{1}$ Wageningen University, Department of Agrotechnology \& Food Sciences, The Netherlands

${ }^{2}$ Wageningen University, Department of Animal Sciences, The Netherlands

${ }^{3}$ Department of Agricultural Sciences, University of Naples "Federico II", Italy
}

Molecular Nutrition \& Food Research 2019: 63 (3) 


\begin{abstract}
Many dietary phytochemicals have been reported to promote gut health. Specific dietary phytochemicals, such as luteolin, as well as specific microbial metabolites of tryptophan are ligands of the Aryl hydrocarbon Receptor (AhR), which plays a role in immunity and homeostasis of the gut barrier. Here, the fate of luteolin during colonic fermentation and the contribution of tryptophan metabolites to AhR activity in different parts of the colon are investigated. Several polyphenols are screened for AhR activation and oregano, containing the ligand luteolin, is added to batch cultures of human microbiota from the distal colon. Luteolin is rapidly metabolized, with no measurable increase in AhR activity. In the second experiment using the Simulator of the Human Intestinal Microbial Ecosystem (SHIME), not all luteolin is metabolized in the ascending colon, but disappears rapidly in the transverse colon. The greatest AhR activity is due to microbiota-derived metabolites of tryptophan, particularly in the descending colon. Luteolin in food is rapidly metabolized in the transverse colon. Tryptophan metabolism by the microbiota in the colon contributes substantially to the pool of lumen metabolites that can activate the AhR.
\end{abstract}

Keywords: Aryl hydrocarbon Receptor, SHIME, Microbiota, Luteolin, Tryptophan 


\section{Introduction}

The Aryl hydrocarbon Receptor (AhR) is best known for its role in detoxification of halogenated aromatic hydrocarbons such as polycyclic aromatic hydrocarbons (PAHs) and dioxins. Of these exogenous compounds, 2,3,7,8-tetrachlorodibenzo-p-dioxin (TCDD) is the most potent activator and it induces diverse toxicological and biological effects ${ }^{1,2}$. Perspectives on the biological role of AhR have changed due to the establishment of a link between dietary or microbial agonists of the AhR and maintenance of host-microbe homeostasis in the intestine ${ }^{3-5}$. Activation of AhR in the gut is essential for maintenance of intraepithelial lymphocytes (IELs) and IL-22 producing innate lymphoid cells (ILC3s), which enhance the gut barrier functions and control the microbial load and composition ${ }^{5,6}$. Therefore, AhR is considered as a sensor that connects the outside environment with cellular processes with consequences for immune functioning ${ }^{5,7,8}$.

The AhR consists of a basic helix-loop-helix (bHLH) protein and belongs to the Per-Arnt-Sim (PAS) superfamily?. The receptor is an intracellular, ligand-activated transcription factor. In the cytosol, AhR forms a complex with two heat shock protein 90 molecules, anX-associated protein 2, prostaglandin E synthase 3 (p23), AhR interacting protein (AIP) and AhR-activated 9 (ARA9) ${ }^{10}$. Upon ligand binding to AhR, AIP is released leading to a conformational change, exposure of the nuclear location signal and translocation to the nucleus. In the nucleus, HSP9O is assumed to dissociate from the complex allowing interaction with ARNT and binding to the dioxin/xenobiotic response elements (DRE/XRE), leading to expression of AhR-regulated genes ${ }^{11}$.

Certain dietary compounds like polyphenols, mainly flavonoids, and tryptophan derivatives have been reported as AhR ligands ${ }^{8,9,12}$. Recent studies showed the major significance of dietary compounds like tryptophan and phytochemicals like indole-derivatives in both intestinal and microbial homeostasis in relation to AhR in mouse colitis models ${ }^{13,14}$. As little is known about how metabolism of these compounds by microbes alters the AhR-mediated signaling activity, we sought to study the kinetics of their release and the ability to activate the AhR using the Simulator of the Human Intestinal Microbial Ecosystem (SHIME), simulating the ascending (AC), transverse (TC), and descending (DC) colon ${ }^{15}$. The aim of our research was to study the effects of dietary ligands on AhR and the effect of microbial fermentation of food matrix on the evolution of the formed ligands. Additionally, we investigated the relative contribution of microbiotaderived tryptophan metabolites to the overall AhR activity in different parts of the colon. 


\section{Materials and Methods}

\subsection{Chemicals}

All chemicals used were purchased from Sigma-Aldrich (St. Louis, MO), unless stated otherwise. The tested polyphenols were: apigenin, baicalein, catechin, caffeic acid, 4-O-caffeoylquinic acid, chrysin, chlorogenic acid, curcumin (Indofine Chemicals (Hillsborough, NJ)), daidzein, epicatechin, epigallocatechin gallate, formononetin, hesperetin, kaempferol, luteolin (Indofine Chemicals), myricetin, naringenin, quercetin, resveratrol (Indofine Chemicals) and rutin (Indofine Chemicals). Two different batches of dried oregano (Origanum vulgare) were purchased on the local marked and used for the batch and SHIME experiments.

\subsection{Luteolin extraction from oregano}

To determine the luteolin concentration in oregano, duplicate samples of $50 \mathrm{mg}$ of milled dried oregano were mixed with $5 \mathrm{~mL}$ of $70 \%(\mathrm{~V} / \mathrm{V})$ methanol and sonicated for $60 \mathrm{~min}$ at $40 \mathrm{kHz}, 100 \mathrm{~W}$ (HBM Machines B.V., Moordrecht, The Netherlands). The temperature during sonication ranged from 22 to $<48^{\circ} \mathrm{C}$. After sonication, the extract was centrifuged at $1363 \mathrm{~g}$ for $15 \mathrm{~min}$ and the supernatant was passed through a $0.20 \mu \mathrm{m}$ cellulose filter (Phenomenex, Torrance, CA) and stored in the dark at room temperature.

\subsection{Batch SHIME}

SHIME (ProDigest, Belgium) was used to mimic the distal colon of three different donors. Microbial inoculum was first stabilized over a period of 2 weeks to adapt to the proximal and distal colon respectively as previously described ${ }^{16}$. Fresh fecal samples were used to inoculate the TRIPLESHIME setup, consisting of a proximal and distal colon for three different donors. The two male and one female fecal stool donors were nonsmoking adults, between 25 and 35 years of age, with no prior history of antibiotic and probiotic use for at least 6 months and 3 weeks respectively. For each donor, three double-jacketed vessels were used, simulating one combined stomach/small intestine, a proximal ( $\mathrm{pH} 5.6$ - 5.9) and a distal colon ( $\mathrm{pH} 6.6$ - 6.9). Every $8 \mathrm{~h}, 70 \mathrm{~mL}$ fresh liquid feed $(\mathrm{pH} 2)$ entered the stomach vessel for each donor with a stable feed composition (1.2 g/L arabinogalactan, $2.0 \mathrm{~g} / \mathrm{L}$ pectin, $0.5 \mathrm{~g} / \mathrm{L}$ xylan, $0.4 \mathrm{~g} / \mathrm{L}$ glucose, $3.0 \mathrm{~g} / \mathrm{L}$ yeast extract, $1.0 \mathrm{~g} / \mathrm{L}$ special peptone, $3.0 \mathrm{~g} / \mathrm{L}$ mucin, $0.5 \mathrm{~g} / \mathrm{L} \mathrm{L-cysteine-HCl}$ and $\left.4.0 \mathrm{~g} / \mathrm{L} \mathrm{starch}{ }^{16}\right)$. After 90 min, $30 \mathrm{~mL}$ of pancreatic juice (12.5 g/L NaHCO 3 ; $6 \mathrm{~g} / \mathrm{L}$ Oxgall, BD Biosciences, The Netherlands; $0.9 \mathrm{~g} / \mathrm{L}$ pancreatin from porcine $\geq 3$ * USP) was added. After 90 minutes of the small intestinal phase, the total volume was transferred to the proximal colon connected in series to the distal colon. The vessel volumes, $\mathrm{pH}$ and retention times were kept constant at all times ${ }^{15,17}$. 
Freshly donated fecal sample was stored in a collection box with an anaerobic AnaeroGen ${ }^{\text {TM }}$ bag (Oxoid, UK), at $4{ }^{\circ} \mathrm{C}$ for less than 8 hours. A $20 \%(\mathrm{~W} / \mathrm{V})$ solution of the fecal sample was homogenized with phosphate buffer for 10 min using a stomacher 400 circulator (Seward, UK). The sterilized phosphate buffer consisted of $8.8 \mathrm{~g} / \mathrm{L} \mathrm{K}_{2} \mathrm{HPO}_{4}$ (Merck KGaA, Germany), $6.8 \mathrm{~g} / \mathrm{L}$ $\mathrm{KH}_{2} \mathrm{PO}_{4}$ (Merck KGaA) and $0.1 \mathrm{~g}$ sodium thioglycolate in demi-water. The $\mathrm{pH}$ was adjusted to 7 and 15 mg sodium thionite (VWR, The Netherlands) was added before use. After mixing, the inoculum was centrifuged for $2 \mathrm{~min}$ at $500 \times \mathrm{g}$ and added in a concentration of $5 \mathrm{~mL}$ per $100 \mathrm{~mL}$ vessel volume. After the 2 weeks stabilization period, the microbiota was collected and stored with $50 \%$ sterilized cryoprotectant (a final concentration of $42 \%$ glycerol, 0.5 g/L cysteine $\mathrm{HCl}$, $10 \mathrm{~g} / \mathrm{L}$ trehalose and $3 \mathrm{~g} / \mathrm{L}$ tryptic soy broth (Oxoid)) at $-80^{\circ} \mathrm{C}$ for further experiments.

The SHIME set-up was modified to study oregano and luteolin fermentation in the distal colon. Three distal colon vessels were used, originating from the three different donors, inoculated with $4 \mathrm{~mL}$ of the frozen stabilized microbiota added to $400 \mathrm{~mL}$ feed per vessel. The microbiota was grown anaerobically overnight, with $\mathrm{pH}$ controlled in the range 6.6 - 6.9, followed by a 3-day program with simulated feedings every $8 \mathrm{~h}$. The experiment included three treatment days, with daily addition of 0.75 grams milled and sieved $(<0.250 \mathrm{~mm}$ ) dried oregano (Greek) per donor to the stomach phase. The concentration of luteolin in oregano was $6.4 \pm 0.3 \mathrm{mg}$ per $100 \mathrm{~g}$. Samples were taken during fermentation and immediately centrifuged for $5 \mathrm{~min}$ at 9000 $x g$ at a temperature of $4{ }^{\circ} \mathrm{C}$. After centrifugation, the supernatants were filtered using a 0.20 $\mu \mathrm{m}$ cellulose filter and stored at $-20^{\circ} \mathrm{C}$ until further analyses. The three donors were used as biological replicates.

\subsection{TWINSHIME}

To simulate a complete microbial fermentation, including an ascending ( $\mathrm{pH} 5.6$ - 5.9, $250 \mathrm{~mL}$ ), transverse ( $\mathrm{pH} 6.15-6.4,400 \mathrm{~mL}$ ) and descending colon ( $\mathrm{pH} 6.6-6.9,300 \mathrm{~mL}$ ), the TWINSHIME was used. Fecal inoculation was performed similarly to the batch SHIME experiment, including a 2-week stabilization period. After stabilization, the microbiota suspension was frozen as described above. The experimental procedure consisted of addition of $1.5 \mathrm{~g}$ dried oregano per donor to the stomach phase, followed by a time series of sampling during fermentation at 10 , 20, 30, 60 and 300 min in all colon parts. This batch of oregano contained $9.7 \pm 0.3 \mathrm{mg}$ luteolin per 100 gram oregano. Individual donors were used as biological duplicates.

\subsection{AhR activation}

Luciferin transfected Dr Chemical Activated LUciferase gene eXpression (CALUX) reporter cells (BioDetection Systems, The Netherlands, mycoplasma free), HepG2 cells and Caco-2 cells were used to measure the AhR activation. 


\subsubsection{Chemical Activated LUciferase gene eXpression}

The reporter cells were grown in a-MEM growth medium (Gibco, USA) with $1 \%$ penicillin/ streptomycin (Gibco) and $10 \%$ heat inactivated fetal calf serum (Gibco), harvested by trypsin/ EDTA and added to white clear bottom 96-wells plate (Corning, USA) at a final concentration of $7.5 \times 10^{4}$ cells per well. After $24 \mathrm{~h}$ incubation, the cells were stimulated with triplicate samples of the polyphenols listed in the chemicals section or controls and incubated for 24 hours before performing the assay. All polyphenols and the positive control were dissolved in DMSO (Merck KGaA, Germany) and 1\% final volume was added to the CALUX reporter cells to measure AhR activation. The microbial culture supernatant was added as $20 \%$ of final volume. After stimulation, the cells were washed twice with $200 \mu \mathrm{L}$ per well PBS, lysed using $20 \mu \mathrm{L}$ per well reporter lysis buffer (Promega, USA), followed by addition of $100 \mu \mathrm{L}$ per well luciferase assay buffer (Promega). The luminescence was measured immediately after adding the assay buffer using a Spectramax M5 (Molecular devices, USA). The results of the AhR activation were expressed as a percentage of the activity obtained with the positive control consisting of $5 \mu \mathrm{M} \beta$-naphthoflavone in $\mathrm{DMSO}^{18}$. All results were corrected for the corresponding negative controls incubated with medium or DMSO $(n=3)$.

\subsubsection{CYP1A1 gene expression}

The HepG2 cells (DSMZ, Germany) were grown in RPMI medium (Gibco) and Caco-2 cells (ATCC, USA) in DMEM medium (Gibco), both with 1\% penicillin/streptomycin and 10\% heat inactivated fetal calf serum. The HepG2 cells were seeded in 12 well plates (Corning) at a density of $\approx 7 \times 10^{5}$ and used 24 hours after seeding. The Caco 2 cells were seeded at a density of $1.5 \times 10^{5}$ in 12 well plates and grown for 2 weeks with regular medium refreshments to allow for differentiation. The cells were stimulated with microbial culture supernatant in a $20 \%$ final volume concentration. After 6 hours incubation, mRNA was extracted using a RNA isolation kit (Qiagen RNeasy mini kit, Germany) including an on-column DNase treatment (Qiagen) and cDNA was synthesized of 1ug mRNA (qScript, QuantaBio, USA). Quantitative PCR (qPCR) was performed using a Rotorgene machine (Qiagen) and SYBRgreen master mix (Promega, USA), to which $5 \mu$ l diluted (1:20) cDNA was added. The primers used were Cyp1A1 fw 5'GACCACAACCACCAAGAAC3'; rv 5'AGCGAAGAATAGGGATGAAG3', GAPDH fw 5'TGCACCACCAACTGCTTAGC3'; rv 5'GGCATGGACTGTGGTCATGAG3', and $\beta$-actin fw 5'GGACTTCGAGCAA GAGATGG3'; rv 5'AGCACTGTGTTGGCGTACAG3'. The program used was $95^{\circ} \mathrm{C}$ for 2 minutes, followed by 40 cycles of $95^{\circ} \mathrm{C}$ for 3 seconds and $60^{\circ} \mathrm{C}$ for 30 seconds. This was followed by a melt curve. The expression of Cyp1A1 was compared to both housekeeping genes and the results were similar following standardization to either gene. "-RT" and non-template controls were performed in every experiment and no amplification above background was detected. The results are shown

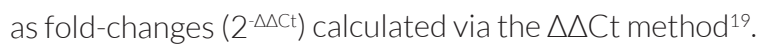




\subsection{Luteolin analysis}

Luteolin concentration was monitored according to Ferracane et al. with some modifications ${ }^{20}$. Flavone was separated using an Ultimate 3000 U-HPLC (Thermo Scientific, Bremen, Germany) equipped with a C18 column (XBridge, $100 \times 2.1$ mm, $2.6 \mu \mathrm{m}$ Waters, UK) and a guard column of the same phase, both thermostated at $30^{\circ} \mathrm{C}$. The binary solvent system consisted of $0.1 \%$ $\mathrm{v} / \mathrm{v}$ formic acid (solvent $\mathrm{A}$ ) and $0.1 \% \mathrm{v} / \mathrm{v}$ formic acid acetonitrile (solvent $\mathrm{B}$ ). The gradient elution was (min/\%B): (0/20), (3/20), (7/80), and (9/80). The flow rate was $300 \mu \mathrm{l} / \mathrm{min}$ and the injection volume $10 \mu \mathrm{l}$. The U-HPLC was interfaced with a TSQ Quantum tandem mass spectrometer equipped with a heated electrospray source (HESI-I, Thermo). Positive selected reaction monitoring (SRM) mode with the following conditions was used: spray voltage $3000 \mathrm{~V}$, sheath gas pressure $10 \mathrm{psi}$, auxiliary gas pressure $5 \mathrm{psi}$ and capillary temperature $260^{\circ} \mathrm{C}$. Luteolin was analyzed by using the mass transitions and collision energy (CE) given in parentheses, in bold the quantitative transition: $\left(\mathrm{m} / \mathrm{z}[\mathrm{M}+\mathrm{H}]^{+}\right.$287/89, CE: $42 \mathrm{~V}$; $\mathbf{2 8 7 / 1 5 3}$, CE: $\left.31 \mathrm{~V}\right)$. Luteolin was quantified using a linear calibration curve with the external standard technique and the results were reported in $\mathrm{ng} / \mathrm{mL}$.

\subsection{Tryptophan metabolites}

Samples were centrifuged $\left(21700 \mathrm{~g}, 10 \mathrm{~min}, 4^{\circ} \mathrm{C}\right.$ ) and diluted five times in $0.1 \%$ formic acid and passed through a $0.22 \mu \mathrm{m}$ cellulose filter (Phenomenex) before high resolution mass spectrometry (HRMS) analysis. Chromatographic separation of tryptophan and tryptophan metabolites was achieved by using an Accela 1250 U-HPLC (Thermo) equipped with a Luna Polar C18 column ( $50 \times 2.1$ mm, $1.6 \mu \mathrm{m}$, Phenomenex) and a guard column of the same phase, both at $40{ }^{\circ} \mathrm{C}$. Mobile phases consisted of $0.1 \% \mathrm{v} / \mathrm{v}$ formic acid (A) and $0.1 \% \mathrm{v} / \mathrm{v}$ formic acid in acetonitrile (B) with the following gradient (min/\%B): (0/2), (0.50/2), (9.5/70), and (12/70). The flow rate was $200 \mu \mathrm{l} / \mathrm{min}$, the column temperature was $40^{\circ} \mathrm{C}$ and $5 \mu \mathrm{l}$ was injected. The U-HPLC system was interfaced to an Exactive Orbitrap HRMS (Thermo) and the analytes were detected through a heated electrospray interface (HESI-II) in positive mode. The current ion of each analyte listed in Table S1 was scanned in the $\mathrm{m} / \mathrm{z}$ range of 50 - 400. The resolving power was set to 75000 full width at half maximum (FWHM, m/z 200) resulting in a scan time of $1 \mathrm{~s}$. The interface parameters were: spray voltage 4.8 kV, capillary voltage $20.0 \mathrm{~V}$, capillary temperature $295^{\circ} \mathrm{C}$, heater temperature $250^{\circ} \mathrm{C}$, and sheath gas flow and auxiliary gas flow were 30 and 9 arbitrary units, respectively. HRMS conditions were optimized by infusing a mixture of indole, indole-3-acetic acid, tryptophan and L-kynurenine $(20 \mu \mathrm{g} / \mathrm{mL})$ at a flow rate of $3 \mu \mathrm{l} / \mathrm{min}$. Analyte concentrations were monitored by using the external standard technique while mass tolerance was set to $5 \mathrm{ppm}$. Three sets of calibration curves for tryptophan, indole, indole-3-propionic acid, indole-3-carboxyaldehyde, indole-3-acetic acid, L-kynurenine, kynurenic acid, tryptamine, 3-hydroxyanthranilic acid, anthranilic acid and 6-formylindolo(3,2-b)carbazole (FICZ) were built in the range $0.455-911 \mathrm{ng} / \mathrm{mL}$. Intraday and interday assays were performed by monitoring three sets of calibration curves within the same day and in three different days. The slope among 
the calibration curves was compared to each replicate and the results were expressed as relative standard deviation RSD (\%). Each sample was analyzed in triplicate and the concentrations given in nM. A summary of the analytical performances of the method is reported in the supporting information (Table S1), while the repeatibility and reproducibility tests were always below 10 $\%$ in the linearity range.

\subsection{Microbial analysis}

$16 \mathrm{~S}$ sequencing of the $\mathrm{V} 3-\mathrm{V} 4$ region was performed by BaseClear (Leiden, The Netherlands) and the results were analyzed using the CLC bio genomics workbench (Qiagen, The Netherlands), Microbial Genomics Toolbox. The SILVA 16S v128 $99 \%$ database was used as reference database. Results show the relative abundances at phylum level for the three different donors.

\subsection{Statistical analyses}

The statistical analyses were performed using GraphPad Prism 5 (GraphPad Software, La Jolla, CA). Results are shown as mean \pm standard error of the mean (SEM). ${ }^{*} p<0.05,{ }^{* *} p<0.01$ and ${ }^{* * *} p<0.001$ are considered statistical differences. Letters above bars represent classes of statistically significant different responses compared to each concentration. Each graph bar with the same letter is not statistically different. The AhR activation data and luteolin concentrations between the different fermentation time points were tested using a one-way ANOVA followed by a Tukey post-hoc analysis. A first order kinetics model was fitted to the luteolin degradation data in order to determine the rate constants for luteolin degradation on each of the three days using the Solver tool in Excel. Differences between days in fermentation rates were analyzed using a one-way repeated measures ANOVA followed by a Tukey post-hoc analysis. Differences between AC, TC and DC in AhR activation were tested using repeated measures two-way ANOVA followed by a Bonferroni multiple comparisons test. The qPCR data was analyzed with ANOVA followed by a Tukey post-hoc analysis using the ddCt values. The heat map was calculated using OMICs (XLSTAT, Addinsoft, NY). Dendrograms were built by the agglomerative hierarchical clustering algorithm considering the concentrations (nM) of each tryptophan metabolite ${ }^{21}$. 


\section{Results and Discussion}

\subsection{Screening dietary phenolic compounds for AhR activation}

Among all polyphenols listed in the chemical section tested for AhR activation, only luteolin, baicalein and 4-O-caffeoylquinic acid showed a dose dependent AhR activation with an optimal concentration of 80,320 and $40 \mu \mathrm{M}$, respectively (Figure 1). Interestingly, quercetin did not activate AhR, and actually reduced AhR activation by luteolin, suggesting an antagonistic effect of quercetin on AhR activation. When luteolin was combined with the ligand $\beta$-naphthoflavone (Figure 1f), no differences in AhR activation were found, suggesting that luteolin does not show antagonistic effects on AhR when combined with another agonist. Chlorogenic acid also did not activate AhR, unlike its isomer 4-O-caffeoylquinic acid. Several studies have reported conflicting results on the capacity of polyphenols to activate $A h R^{12,22-25}$, most likely due to use of different cell types in the reporter assay ${ }^{26}$. Baicalein has been reported to activate AhR using different cell lines, but quercetin is often reported as either a (weak) ligand or antagonist ${ }^{27}$, consistent with our results (Figure 1e).
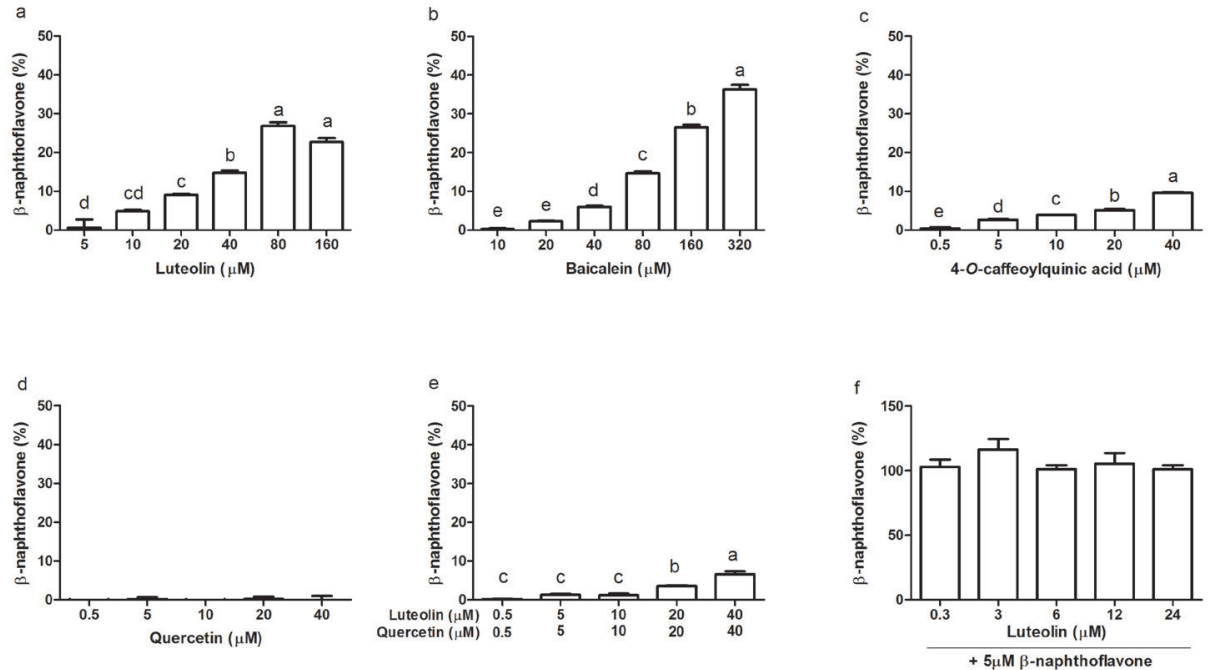

Figure 1. AhR activation measured with the CALUX reporter assay with luciferase production as readout, expressed as percent of the positive control ( $\beta$-naphthoflavone, $5 \mu \mathrm{M})$. (a) Luteolin; (b) Baicalein; (c) 4-O-caffeoylquinic acid; (d) Quercetin (e) Luteolin combined with an equivalent concentration of quercetin; (f) Luteolin combined with $5 \mu \mathrm{M} \beta$-naphthoflavone. Letters above the bar represent classes of statistically significant different responses compared to each concentration. Bars with the same letter are not statistically different. 
Luteolin and baicalein belong to a sub-class of flavonoids named flavones. In their most stable conformation, flavone conformers can take on a planar structure, which is thermodynamically unfavorable to flavonols, because of the presence of a hydroxyl group at C3 position of the $\mathrm{C}$ carbon ring. Based on these observations and the fact that several known AhR ligands are planar (e.g. $\beta$-naphthoflavone dioxin, FICZ), we speculate that a planar structure is an important structural feature of AhR ligands. Despite the fact that quercetin differs from luteolin by a single hydroxyl group, on C3 of the $\mathrm{C}$ ring, quercetin did not activate the AhR receptor in our assay. This is consistent with the fact that the most energetically stable conformer of quercetin is not a planar structure. Clearly, structural features other than the planar conformation are important for the activation of AhR because other flavones such as apigenin, which possesses a planar structure, did not activate AhR. The structure-dependent AhR activation by flavonoids is also described by Jin et al., where they show that the number of hydroxyl groups plays an important role in AhR activity 26 .

Among the compounds which activate AhR, luteolin has dietary relevance as it is being found in several food sources such as oregano and parsley ${ }^{28}$. Baicalein has been found in the roots of the Scutellariae baicalensis. This root is not consumed as a food, but frequently used in Chinese herbal medicine ${ }^{29}$, while 4-O-caffeoylquinic acid is present in foods but its relative capacity to activate AhR is low. For these reasons, we decided to use oregano in our batch fermentation study because it is a rich source of luteolin.

\subsection{Batch fermentation of oregano}

To study the metabolic fate of luteolin in oregano during distal colon fermentation, a batch fermentation was performed where 0.75 grams of oregano per donor was added to the stomach phase of the digestion. The batch fermentation used in this experiment is a more physiological representation of human fermentation in vivo than conventional batch fermentation studies. After $10 \mathrm{~min}$, the entire content of the small intestinal vessel was transferred directly to a vessel containing the microbiota of the distal colon. Results in Figure 2a indicated that, immediately after transfer, luteolin was rapidly metabolized by the microbiota of all donors. The luteolin bioaccessibility on day 1 was $179 \%$, followed by $122 \%$ at day 2 and $149 \%$ at day 3 . This showed that luteolin was readily and fully bio-accessible from the finely grounded plant matrix that was used. After 60 min of fermentation, almost all luteolin was metabolized. Significant differences were observed in the rate of degradation of luteolin from oregano on different days (Figure 2b).

On day 3 , luteolin was more rapidly degraded than on day 1 ( $p<0.05$, Table 1 ), which may be due to a metabolic adaptation or a shift in the microbiota composition at the lower taxonomic levels, in response to daily addition of polyphenols. Labib et al. performed a batch fermentation of luteolin with pig microbiota, where luteolin was broken down and the single metabolite 3-(3-hydroxyphenyl)-propionic acid was formed ${ }^{30}$. Given its structural similarity to caffeic acid, there is no indication that the metabolite can activate AhR. In their study, the rate of luteolin 
degradation was lower than that measured in our human colon fermentation model. This might be due to differences in microbial density or composition, fermentation conditions or different concentrations of luteolin ${ }^{31}$.

a

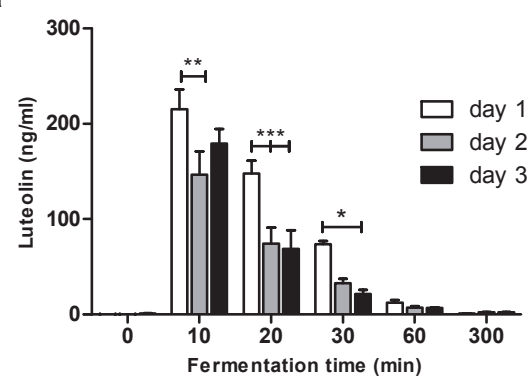

b

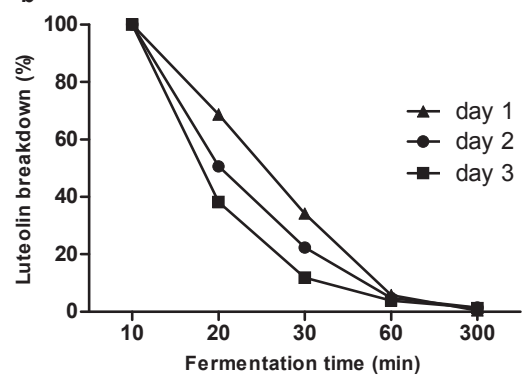

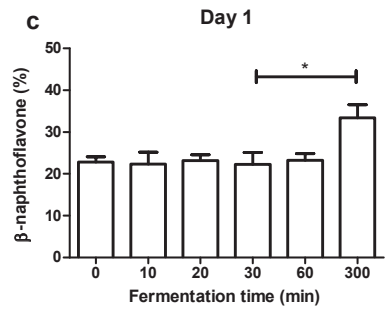
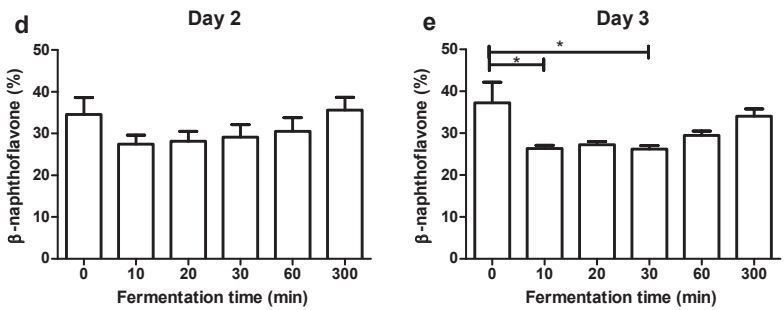

Figure 2. (a) Luteolin concentration in distal colon supernatants over 300 min on three consecutive days of oregano feeding, $\mathrm{n}=3$ donors. (b) Rate of luteolin breakdown rate as percentage of starting concentration on three consecutive days of oregano feeding, $\mathrm{n}=3$ donors. (c-e) AhR activation in the distal colon on each day of consecutive oregano feeding, measured with the CALUX reporter assay using luciferase production as readout. Results are expressed as percent of the positive control ( $\beta$-naphthoflavone, $5 \mu \mathrm{M}), n=3$ donors. Data are expressed as mean of three donors \pm SEM, with ${ }^{*} p<0.05,{ }^{* *} p<0.01$, and ${ }^{* * *} p<0.001$.

Interestingly, a similar breakdown kinetics of luteolin and AhR activation capacity was found in all three donors so their microbial composition was compared. The microbial composition in the distal colons after the stabilization period of the three different donors is depicted in Figure 3. Donor 2 shows a different Firmicutes/Bacteroidetes ratio (1.33 compared to 0.32 and 0.39 for resp. donor 1 and 3). Donor 2 also shows a higher relative abundance in Actinobacteria compared to donor 1 and 3. The relative abundance of Proteobacteria was similar for all donors. Differences of this magnitude are typical of the normal variation observed between individuals, including differences due to gender ${ }^{32}$. In our study, the use of a standardized diet in the system throughout the stabilization period and experiments could have reduced the variability. However, even after a 2 weeks stabilization period with this diet, the microbial communities between donors preserve the original differences (data not shown). 
Table 1. Rate constants ( $\mathrm{min}^{-1}$ ) of luteolin degradation over three consecutive days of oregano feeding.

\begin{tabular}{lccc}
\hline & Day 1 & Day 2 & Day 3 \\
\hline Donor 1 & 0.0516 & 0.0785 & 0.1138 \\
Donor 2 & 0.0413 & 0.0689 & 0.1236 \\
Donor 3 & 0.0559 & 0.0695 & 0.0789 \\
\hline
\end{tabular}

Data of Figure 2c-e showed that supernatants from the colonfermentation have a significant capacity to activate AhR (between 20 and $30 \%$ of the reference). However, AhR activity does not change for up to 60 minutes after oregano addition, at which point the luteolin has been completely degraded (see Figure 2a), suggesting that at this concentration luteolin does not significantly contribute to the AhR activity present in the supernatants. Indeed, similar results were obtained with the microbial supernatant from the fermenter to which no oregano was added (Time 0, Figure 2c-e), demonstrating that substantial amounts of AhR ligands were being produced by the fecal microbiota.

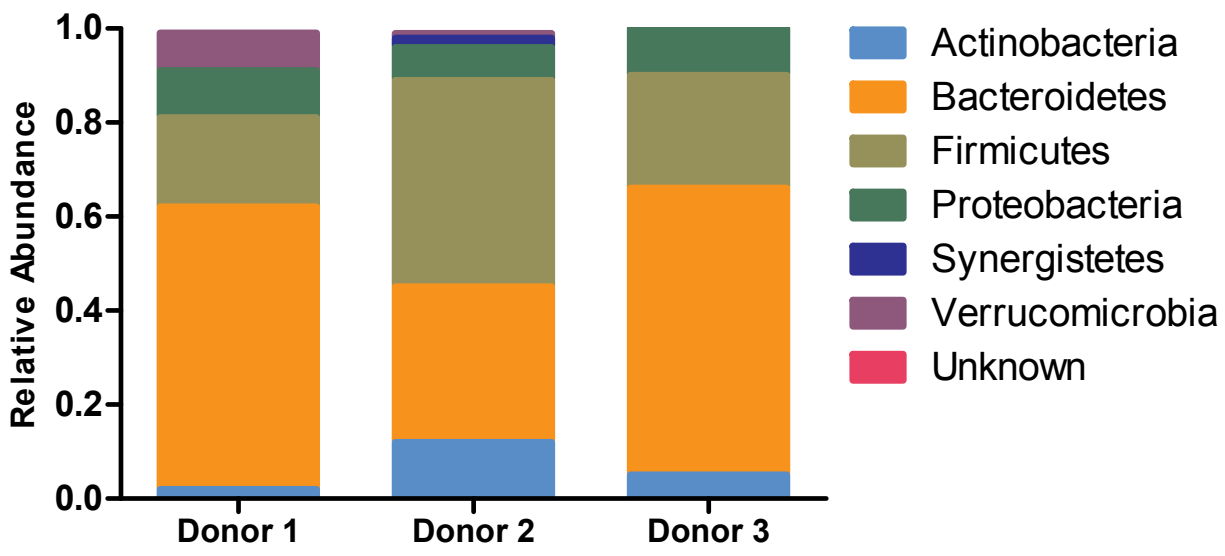

Figure 3. Microbiota composition at phylum level (relative abundance) for the three different donors used in the distal colon batch fermentation. Microbiota composition was determined after the 2-week stabilization period in the SHIME and before addition of oregano.

\subsection{TWINSHIME fermentation oregano}

To assess the metabolic fate of dietary luteolin in connection to AhR activation in the different parts of the lower gut, a SHIME fermentation study on oregano was performed. $1.5 \mathrm{~g}$ of oregano per donor was added to the stomach/small intestinal phase, after which the content of the stomach/small intestinal vessel was automatically transferred in series to the vessels simulating the AC, TC and DC. The two different donors used in the SHIME study showed a different microbial composition (Figure S1) with comparable functionality, similar to the previous experiment. Therefore, results are shown as biological duplicates. 
Figure 4 shows the luteolin concentration over time in the AC (Figure 4a), TC (Figure 4b) and DC (Figure 4c). The most interesting finding was the limited metabolism of luteolin in the AC after 60 minutes (20.5\%, p > 0.05), which was reduced to $53.3 \%$ after $300 \mathrm{~min}(\mathrm{p}<0.01)$. In contrast, a rapid decrease of luteolin was observed in the TC. The concentration of luteolin observed in the TC was initially lower than in the AC due to dilution upon transfer to the TC vessel, reaching a peak value of $\approx 100 \mathrm{ng} / \mathrm{mL}$ at $30 \mathrm{~min}$, when the transfer was complete, after which it rapidly decreased. In the DC, low amounts of luteolin were present at 30 min due to its degradation in the TC and dilution upon transfer. Overall, these results suggest that breakdown of luteolin occurs primarily in the TC. The differential luteolin breakdown ability of the microbiota in the different part of the gut is a new finding that could have various biological implications. This evidence could be likely extended also to other polyphenols. In fact, the recent study of Wu et al. showed that absorption of polyphenols is influenced by location in the colon, with generally more absorption in the DC. Their data showed high recovery of polyphenols in the AC, whereas in the TC this is limited ${ }^{33}$.

Supernatants from each colon condition were tested for their capacity to activate AhR during the 300 min fermentation of oregano (Figure 4d). All supernatants strongly activated AhR with highest activity in the DC where no luteolin was present. These results are comparable to the qPCR data where the Cyp1A1 gene expression is higher in the DC compared to the TC and AC in both human liver (HepG2) and intestinal (Caco-2) cells (Figure 5). It should be noted that the concentration of luteolin found in all the SHIME compartments is too low to account for the AhR signaling activity. The maximum recovered luteolin concentration was about $2 \mu \mathrm{M}$ in the AC, which is about 20 times lower than what is needed to measure a significant AhR activation. The fermentation medium alone had no AhR activity and the luteolin released from oregano makes only a minor contribution to the total activity, indicating that the microbiota produces AhR activating ligands from other sources.

Figure 6 shows the AhR activation in the AC. The basal activation is much lower than in the DC and a significant increase was observed after oregano feeding compared to a non-oregano feeding control. Oregano induces AhR activation in the AC, which is comparable to the base level AhR activation in the DC (Figure 4d). These results suggested that food components contribute to the total amount of AhR agonists present in the proximal part of the colon but the vast majority of AhR ligands present in the TC and DC are produced by the microbiota. We can hypothesize that there is an optimal production of microbial-derived AhR ligands along the colon and other AhR activators are needed when the microbial metabolites are not sufficiently present. This gap can be filled by dietary compounds ${ }^{34}$. Which exact compounds trigger the AhR receptor in the AC in this case remains unknown, but luteolin alone cannot be responsible for this effect. 

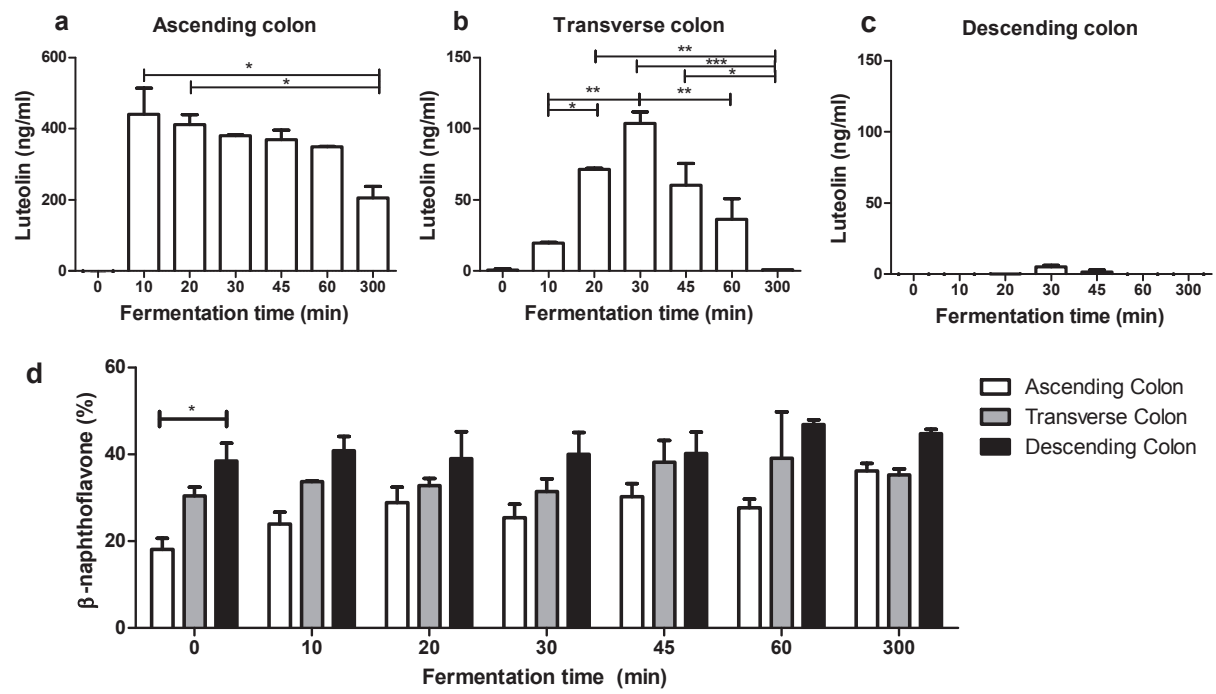

Figure 4. (a-c) The luteolin concentration after feeding oregano to the SHIME system, presenting a partially-dynamic system of ascending (a), transverse (b) and descending (c) colon connected in series. $n=2$ donors. (d) AhR activation measured with the CALUX reporter assay in the ascending, transverse and descending colon during fermentation after addition of $1.5 \mathrm{~g}$ oregano per donor ( $n=2$ donors), using luciferase production as readout, expressed as percent of the positive control ( $\beta$-naphthoflavone, $5 \mu \mathrm{M}$ ). Data are expressed as mean of two donors \pm SEM, with ${ }^{*} p<0.05,{ }^{* *} p<0.01$, and ${ }^{* * *} p<0.001$.
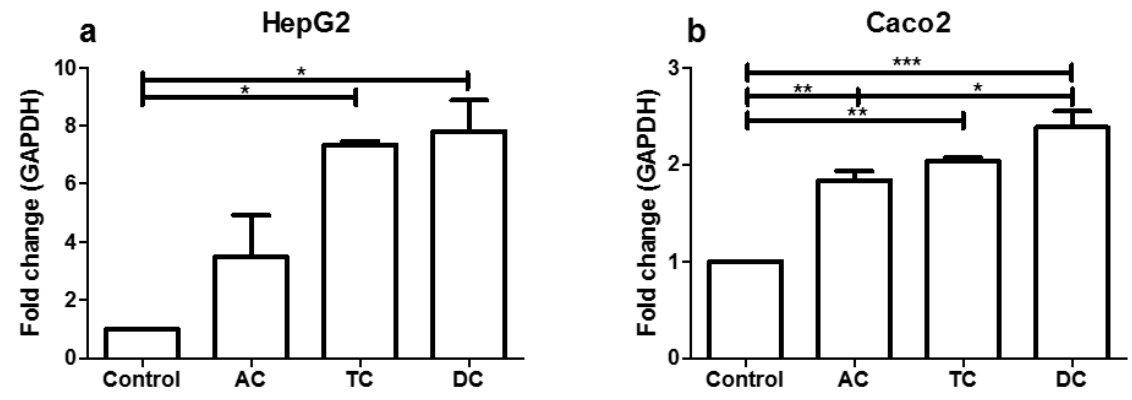

Figure 5. QPCR of Cyp1A1 gene expression as fold change compared to housekeeping gene GAPDH after stimulation of (a) HepG2 and (b) Caco-2 cells with SHIME samples from ascending colon (AC), transverse colon (TC) and descending colon (DC) of donor 1 and 2. The experiment was repeated twice for both donors, with ${ }^{*} p<0.05,{ }^{* *} p<0.01$, and ${ }^{* * *} p<0.001$. 


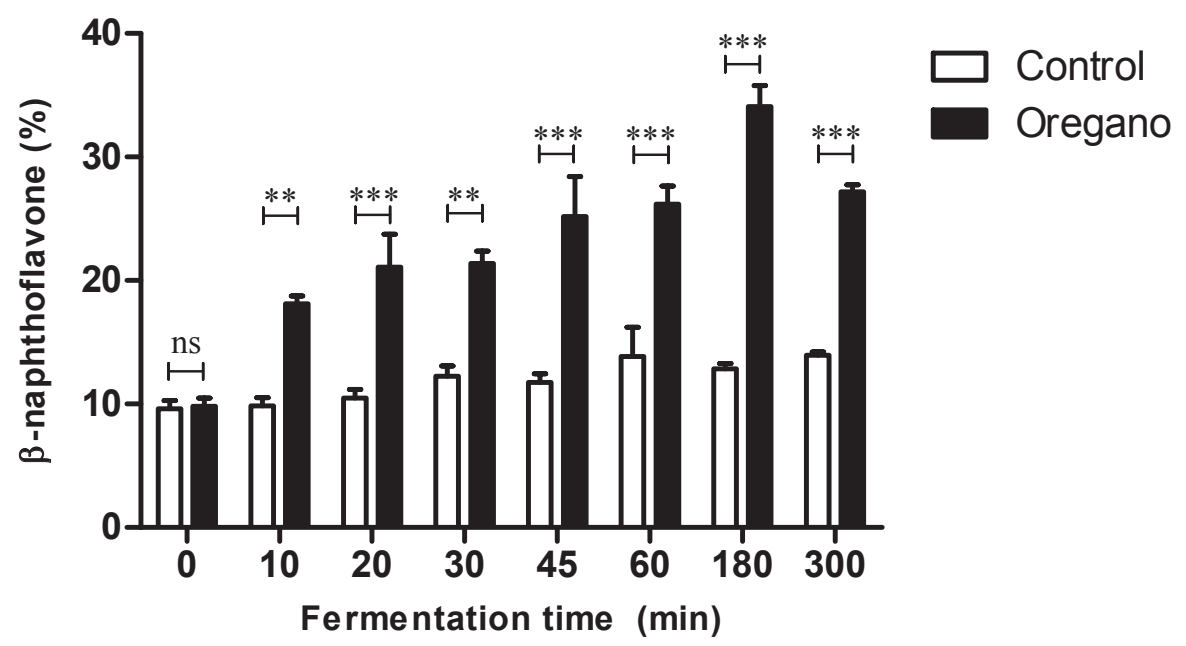

Figure 6. AhR activation in the ascending colon for a control fermentation compared to oregano fermentation, measured with the CALUX reporter assay using luciferase production as readout, expressed as percent of the positive control ( $\beta$-naphthoflavone, $5 \mu \mathrm{M}), n=2$ donors. Data are expressed as mean of two donors \pm SEM, with ${ }^{*} p<0.05,{ }^{* *} p<0.01$, and ${ }^{* * *} p<0.001$.

\subsection{Role of tryptophan derivatives in AhR activation}

We hypothesized that a microbial source of AhR agonists in the SHIME supernatants were derived from the metabolism of tryptophan in the SHIME basal feed. The metabolism of tryptophan by certain gut bacteria has been shown to generate indole metabolites that exhibit both AhR agonistic and partial antagonistic activities ${ }^{8,35-38}$. Furthermore, tryptophan supplementation in the diet of mice resulted in the production of tryptophan metabolites which attenuated colitis in an AhR-dependent fashion ${ }^{14}$.

Results of measuring tryptophan metabolites (Table S1, Supporting Information) in fermentation supernatants are summarized in Figure 7 and their concentrations are given in Figure S2, Supporting Information. Ward's method was used to scale, center and cluster data in order to depict hierarchical relationship between microbiota and the compounds formed ${ }^{21}$. Colon sections of the two donors were grouped together as revealed by the horizontal dendrogram, confirming the similar metabolic capacity of microbiota of different donors. Metabolites were clustered into three main groups according to the concentration in the three different sections. Anthranilic acid, tryptamine and 3-(2-hydroxyethyl)indole exhibited a constant concentration through the sections, while the other metabolites showed increased or decreased concentrations through the sections. In the bottom left corner of the dendrogram (AC1 AC2; colored red), the metabolites with highest concentration in the AC were tryptophan, L-kynurenine, indole-3- 
aldehyde, 3-hydroxyanthranilic acid, 3-methylindole and indole-3-acetic acid. However, these were measured in substantially lower amounts in the DC supernatants suggesting ongoing metabolism or degradation as they move through the colon. Oxindole, indole, kynurenic acid, indole-3-acetaldehyde and indole-3-propionic acid were present at a higher concentration in the DC than in the AC supernatants. The tryptophan concentration changed from $3417 \pm 100$ $\mathrm{nM}$ in the AC, to a concentration of $372 \pm 26$ and $291 \pm 0.9 \mathrm{nM}$ in the TC and DC, respectively, while for instance indol-3-acetaldehyde increases from $3 \pm 0.4 \mathrm{nM}$ in the AC to $14 \pm 0.0$ and 16 $\pm 0.0 \mathrm{nM}$ in the TC and DC, respectively. The tryptophan derivatives, kynurenine, tryptamine, indole-3-acetaldehyde, indole-3-acetic acid and indole-3-aldehyde are known AhR ligands ${ }^{8}$, explaining why the greatest AhR activation is found in the DC supernatants (Figure 4b).

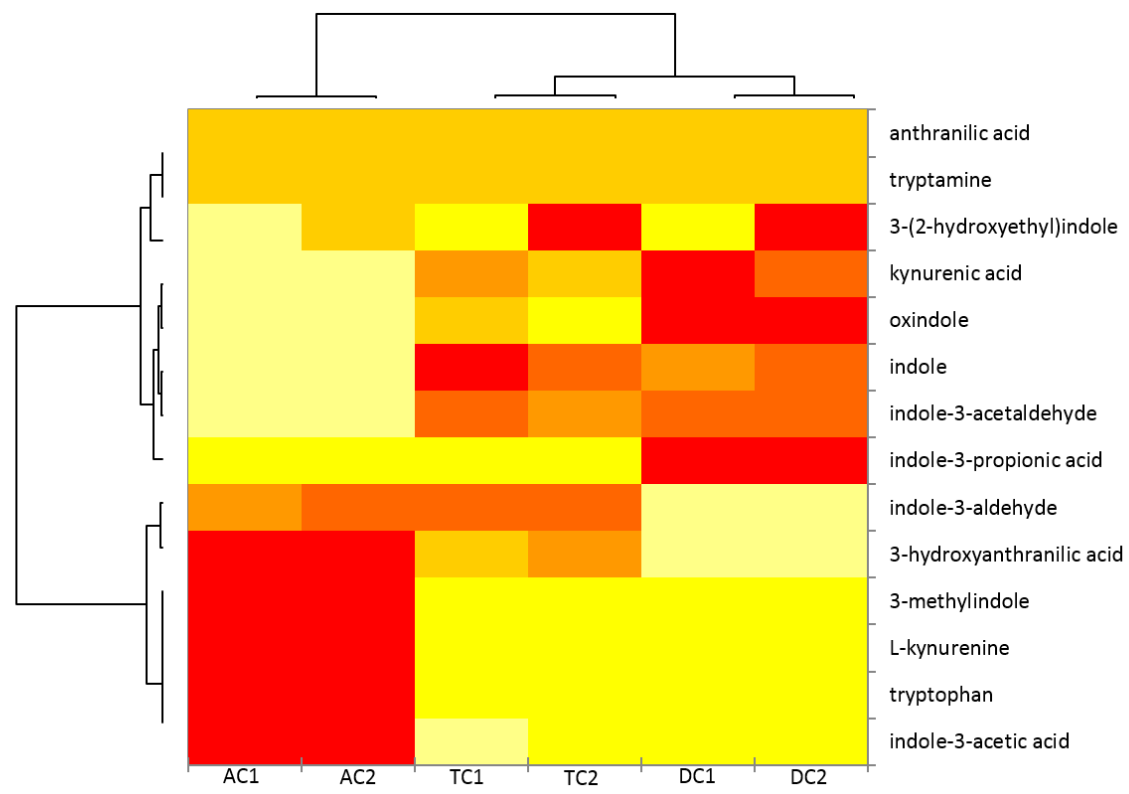

Figure 7. Heat map of tryptophan and tryptophan derivatives, from yellow (lower concentration) to red (higher concentration). Ascending colon (AC), transverse colon (TC) and descending colon (DC) of donor 1 and 2. The concentrations (nM) are reported in Figure S2, Supporting Information.

An intrinsic limitation of the SHIME model system is that it does not model the intestinal absorption ${ }^{16}$. As the intermediate tryptophan derivatives are not constantly removed as happens in vivo, an overestimation of AhR activity in the lumen of the human colon is expected because high levels of tryptophan are usually not reached in the distal colon. Besides lack of absorption, there is a discontinuous flow of liquids between colon sections in the system, resulting in a stricter cut-off between colon sections compared to in vivo where there is a continuous flow of material along the $\mathrm{AC}, \mathrm{TC}$ and $\mathrm{DC}^{39}$. 


\section{Concluding remarks}

Our results with the food component oregano demonstrated that AhR activity in the large intestine can be modulated by dietary compounds. Polyphenols with a planar structure were generally found to be good AhR ligands. Luteolin, an AhR agonist present in oregano, is unlikely to achieve significant AhR activation along the entire colon due to its rapid degradation. However, other dietary AhR ligands in oregano were shown to activate AhR in the ascending colon. Tryptophan metabolites generated by the microbiota are found in relatively high concentration in all colon parts in our fermentation model, some of which are potent AhR ligands. Overall, it can be concluded that there is a dynamic formation of AhR ligands originating from dietary tryptophan by the gut microbiota and that other dietary AhR ligands can act as complementary AhR activators in the ascending colon.

\section{Acknowledgements}

Research presented in this publication was financially supported by the Graduate School VLAG. Geert Meijer is gratefully acknowledged for setting up the LC-MS/MS method for detecting luteolin, Erik Meulenbroeks for his assistance operating the SHIME, Carien Voogt for her microbial analysis work, and Jorn Assmann for some of the polyphenol CALUX assays.

\section{Conflicts of interest}

The authors declare no conflicts of interest. 


\section{References}

1 White, S. S. \& Birnbaum, L. S. An overview of the effects of dioxins and dioxin-like compounds on vertebrates, as documented in human and ecological epidemiology. Journal of Environmental Science and Health, Part C 27, 197-211, (2009).

2 Denison, M. S., Pandini, A., Nagy, S. R., Baldwin, E. P. \& Bonati, L. Ligand binding and activation of the Ah receptor. Chemico-Biological Interactions 141, 3-24, (2002).

3 Kiss, E. A. et al. Natural aryl hydrocarbon receptor ligands control organogenesis of intestinal lymphoid follicles. Science 334, 1561-1565, (2011).

4 Murray, I. A., Nichols, R. G., Zhang, L., Patterson, A. D. \& Perdew, G. H. Expression of the aryl hydrocarbon receptor contributes to the establishment of intestinal microbial community structure in mice. Scientific Reports 6, 33969, (2016).

$5 \mathrm{Li}$,Y.etal. Exogenous stimuli maintain intraepithelial lymphocytes via aryl hydrocarbon receptor activation. Cell 147, 629-640, (2011).

6 Qiu, J.et al. The aryl hydrocarbon receptor regulates gut immunity through modulation of innate lymphoid cells. Immunity 36, 92-104, (2012).

7 Lee, J. S. et al. AHR drives the development of gut ILC22 cells and postnatal lymphoid tissues via pathways dependent on and independent of Notch. Nature Immunology 13, 144, (2012).

8 Zelante, T.et al. Tryptophan Catabolites from Microbiota Engage Aryl Hydrocarbon Receptor and Balance Mucosal Reactivity via Interleukin-22. Immunity 39, 372-385, (2013).

9 Denison, M.S. \& Nagy, S. R. Activation of the aryl hydrocarbon receptor by structurally diverse exogenous and endogenous chemicals. Annual Review of Pharmacology and Toxicology 43, 309-334, (2003).

10 Carlson, D. B. \& Perdew, G. H. A dynamic role for the Ah receptor in cell signaling? Insights from a diverse group of Ah receptor interacting proteins. Journal of Biochemical and Molecular Toxicology 16, 317-325, (2002).

11 Safe, S. \& Wormke, M. Inhibitory Aryl Hydrocarbon Receptor- Estrogen Receptor a Cross-Talk and Mechanisms of Action. Chemical Research in Toxicology 16, 807-816, (2003).

12 Amakura, Y. et al. Influence of food polyphenols on aryl hydrocarbon receptor-signaling pathway estimated by in vitro bioassay. Phytochemistry 69, 3117-3130, (2008).

13 Hubbard, T. D. et al. Dietary broccoli impacts microbial community structure and attenuates chemically induced colitis in mice in an Ah receptor dependent manner. Journal of Functional Foods 37, 685-698, (2017).

14 Islam, J. et al. Dietary tryptophan alleviates dextran sodium sulfate-induced colitis through aryl hydrocarbon receptor in mice. The Journal of Nutritional Biochemistry 42, 43-50, (2017).

15 Molly, K., Woestyne, M. V.\& Verstraete, W. Development of a 5-step multi-chamber reactor as a simulation of the human intestinal microbial ecosystem. Applied Microbiology and Biotechnology 39, 254-258, (1993).

16 Possemiers, S., Verthé, K., Uyttendaele, S. \& Verstraete, W. PCR-DGGE-based quantification of stability of the microbial community in a simulator of the human intestinal microbial ecosystem. FEMS Microbiology Ecology 49, 495-507, (2004).

17 Molly, K., Woestyne, M. V., Smet, I. D. \& Verstraete, W. Validation of the simulator of the human intestinal microbial ecosystem (SHIME) reactor using microorganism-associated activities. Microbial Ecology in Health and Disease 7, 191-200, (1994).

18 Bradshaw, T. D. \& Bell, D. R. Relevance of the aryl hydrocarbon receptor (AhR) for clinical toxicology. Clinical Toxicology 47, 632-642, (2009). 
19 Pfaffl, M. W. A new mathematical model for relative quantification in real-time RT-PCR. Nucleic Acids Research 29, e45-e45, (2001).

20 Ferracane, R., Graziani, G., Gallo, M., Fogliano, V. \& Ritieni, A. Metabolic profile of the bioactive compounds of burdock (Arctium lappa) seeds, roots and leaves. Journal of Pharmaceutical and Biomedical Analysis 51, 399-404, (2010).

21 Ward Jr, J. H. Hierarchical grouping to optimize an objective function. Journal of the American Statistical Association 58, 236-244, (1963).

22 Ashida, H., Nishiumi, S. \& Fukuda, I. An update on the dietary ligands of the AhR. Expert Opinion on Drug Metabolism \& Toxicology 4, 1429-1447, (2008).

23 Fukuda, I., Mukai, R., Kawase, M., Yoshida, K.-i. \& Ashida, H. Interaction between the aryl hydrocarbon receptor and its antagonists, flavonoids. Biochemical and Biophysical Research Communications 359 822-827, (2007).

24 Medjakovic, S., Mueller, M. \& Jungbauer, A. Potential Health-modulating Effects of Isoflavones and Metabolites via Activation of PPAR and AhR. Nutrients 2, 241-279, (2010).

25 Busbee, P. B., Rouse, M., Nagarkatti, M. \& Nagarkatti, P. S. Use of natural AhR ligands as potential therapeutic modalities against inflammatory disorders. Nutrition Reviews 71, 353-369, (2013).

$26 \mathrm{Jin}$, U. H. et al. Structure-Dependent Modulation of Aryl Hydrocarbon Receptor-Mediated Activities by Flavones. Toxicological Sciences, (2018).

27 Zhang, S., Qin, C. \& Safe, S. H. Flavonoids as aryl hydrocarbon receptor agonists/antagonists: effects of structure and cell context. Environmental Health Perspectives 111, 1877, (2003).

28 Kaefer, C. M. \& Milner, J. A. The role of herbs and spices in cancer prevention. The Journal of Nutritional Biochemistry 19, 347-361, (2008).

29 Bae, M.-J. et al. Baicalein induces CD4+ Foxp3+ T cells and enhances intestinal barrier function in a mouse model of food allergy. Scientific Reports 6, 32225, (2016).

30 Labib, S., Hummel, S., Richling, E., Humpf, H. U. \& Schreier, P. Use of the pig caecum model to mimic the human intestinal metabolism of hispidulin and related compounds. Molecular Nutrition \& Food Research 50, 78-86, (2006).

31 Rechner, A. R. et al. Colonic metabolism of dietary polyphenols: influence of structure on microbial fermentation products. Free Radical Biology and Medicine 36, 212-225, (2004).

32 The Human Microbiome Project, C. Structure, function and diversity of the healthy human microbiome. Nature 486, 207-214, (2012).

$33 \mathrm{Wu}$, T. et al. Aronia (Aronia melanocarpa) phenolics bioavailability in a combined in vitro digestion/

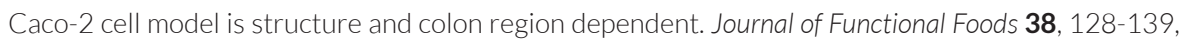
(2017).

34 Schiering, C. et al. Feedback control of AHR signalling regulates intestinal immunity. Nature 542, 242245, (2017).

35 Cervantes-Barragan, L. et al. Lactobacillus reuteri induces gut intraepithelial CD4+ CD8aa+ T cells. Science 357, 806-810, (2017).

36 Keszthelyi, D., Troost, F. \& Masclee, A. Understanding the role of tryptophan and serotonin metabolism in gastrointestinal function. Neurogastroenterology \& Motility 21, 1239-1249, (2009).

37 Hubbard, T. D., Murray, I. A. \& Perdew, G. H. Indole and Tryptophan Metabolism: Endogenous and Dietary Routes to Ah Receptor Activation. Drug Metabolism and Disposition 43, 1522-1535, (2015).

38 Cheng, Y. et al. Aryl hydrocarbon receptor activity of tryptophan metabolites in young adult mouse colonocytes. Drug Metabolism and Disposition 43, 1536-1543, (2015).

39 Cremer, J.et al. Effect of flow and peristaltic mixing on bacterial growth in a gut-like channel. Proceedings of the National Academy of Sciences 113, 11414-11419, (2016). 


\section{Supporting information}

Table S1. HRMS performances. RT (retention time, min); TM (theoretical mass); EM (experimental mass, $\left.[\mathrm{M}+\mathrm{H}]^{+}\right)$; error $(\triangle \mathrm{ppm})$. The following compounds were quantified using chemical similarities toward the analytes in parenthesis (SciFinder, ACS 2018): 3-(2-hydroxyethyl)indole (indole-acetic acid), oxindole (indole), 3-methyl indole (indole), indole-3-acetaldehyde (indole-3-aldehyde).

\begin{tabular}{lccccc}
\hline Compound Name & RT & $\begin{array}{c}\text { Elemental } \\
\text { Composition }\end{array}$ & TM & EM & $\Delta$ ppm \\
\hline L-kynurenine & 2.3 & C10H12N2O3 & 209.09207 & 209.09275 & 3.3 \\
indole-3-acetaldehyde & 2.6 & C10H9NO & 160.07569 & 160.07549 & -1.2 \\
3-hydroxyanthranilic acid & 3.2 & C7H7NO3 & 154.04987 & 154.04991 & 0.3 \\
tryptophan & 4.1 & C11H12N2O2 & 205.09715 & 205.09754 & 1.9 \\
3-methyl indole & 4.3 & C9H9N & 132.08078 & 132.08028 & -3.8 \\
tryptamine & 4.4 & C10H12N2 & 161.10732 & 161.10719 & -0.8 \\
kynurenic acid & 4.6 & C10H7NO3 & 190.04987 & 190.04963 & -1.3 \\
anthranilic acid & 4.9 & C7H7NO2 & 138.05496 & 138.05490 & -0.4 \\
indole-3-aldehyde & 5.5 & C9H7NO & 146.06004 & 146.05990 & -1.0 \\
indole-3-acetic acid & 5.6 & C10H9NO2 & 176.07061 & 176.07021 & -2.3 \\
3-(2-hydroxyethyl)indole & 5.8 & C10H11NO & 162.09134 & 162.09155 & 1.3 \\
indole-3-propionic acid & 6.2 & C11H11NO2 & 190.08626 & 190.08611 & -0.8 \\
indole & 6.7 & C8H7N & 118.06513 & 118.06509 & -0.3 \\
oxindole & 6.8 & C8H7NO & 134.06004 & 134.06022 & 1.3 \\
6-formylindolo(3,2-b)carbazole & 8.6 & C19H12N2O & 285.10224 & 285.10199 & -0.9 \\
\hline
\end{tabular}




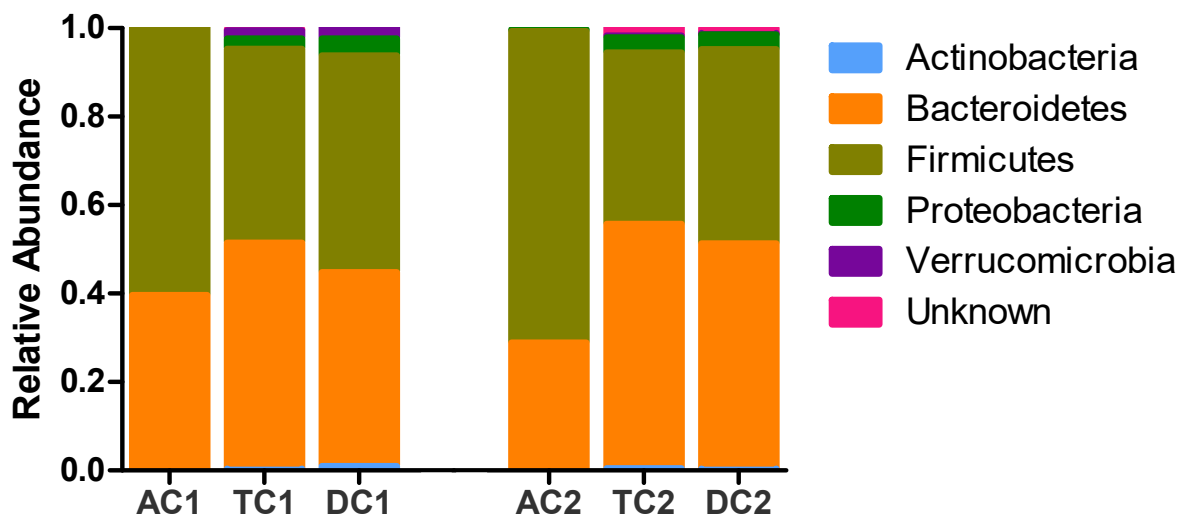

Figure S1. Relative abundance of microbiota used in the TWINSHIME fermentation of 2 different donors before oregano treatment given in phylum level, analyzed by $16 \mathrm{~S}$ sequencing. AC1/AC2 represents ascending colon donor 1/2; TC1/TC2 represents transverse colon donor 1/2; DC1/DC2 represents descending colon donor $1 / 2$. 

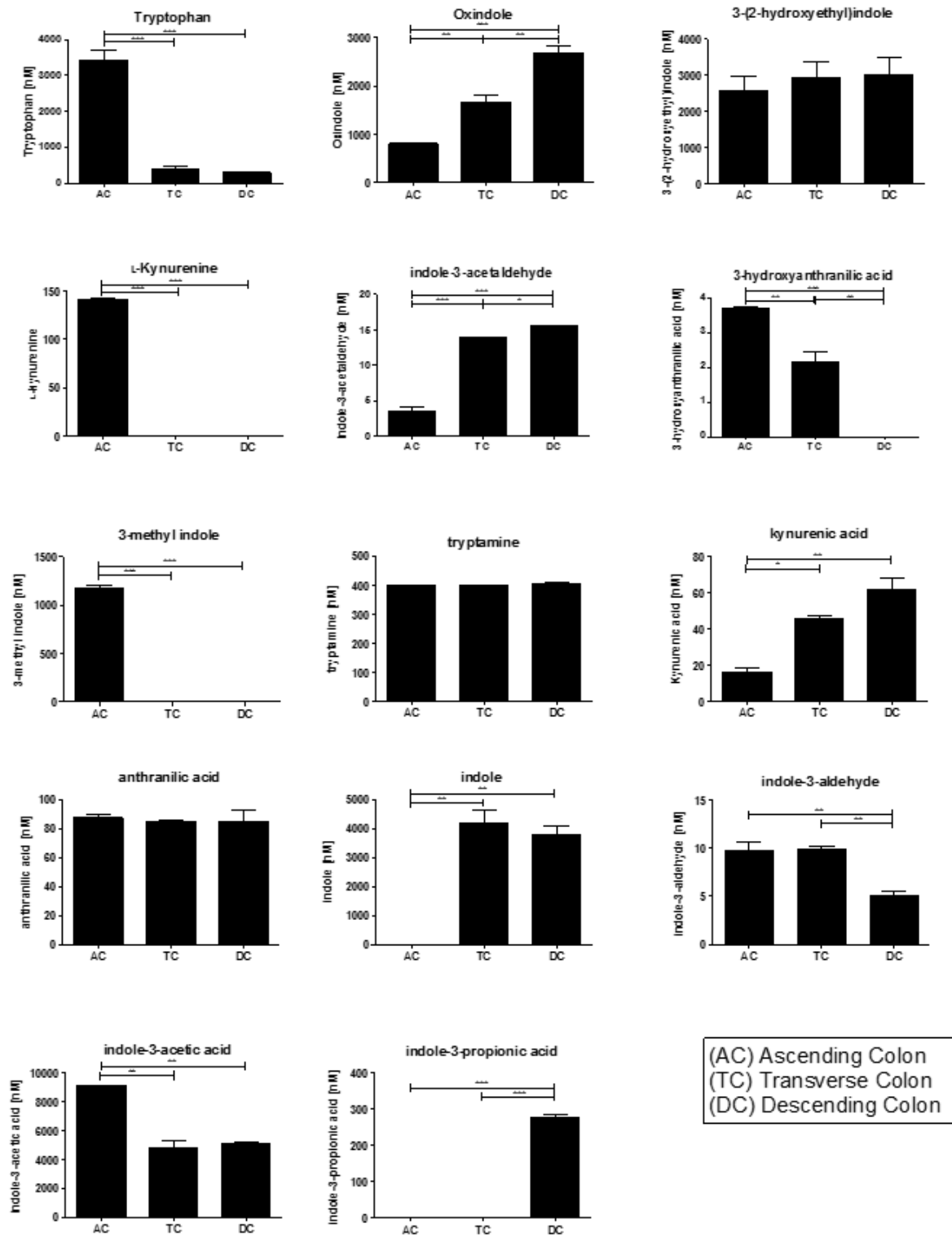

Figure S2. Concentration of tryptophan and tryptophan derivatives in ascending (AC), transverse (TC) and descending (DC) colon, $n=2$ donors. 



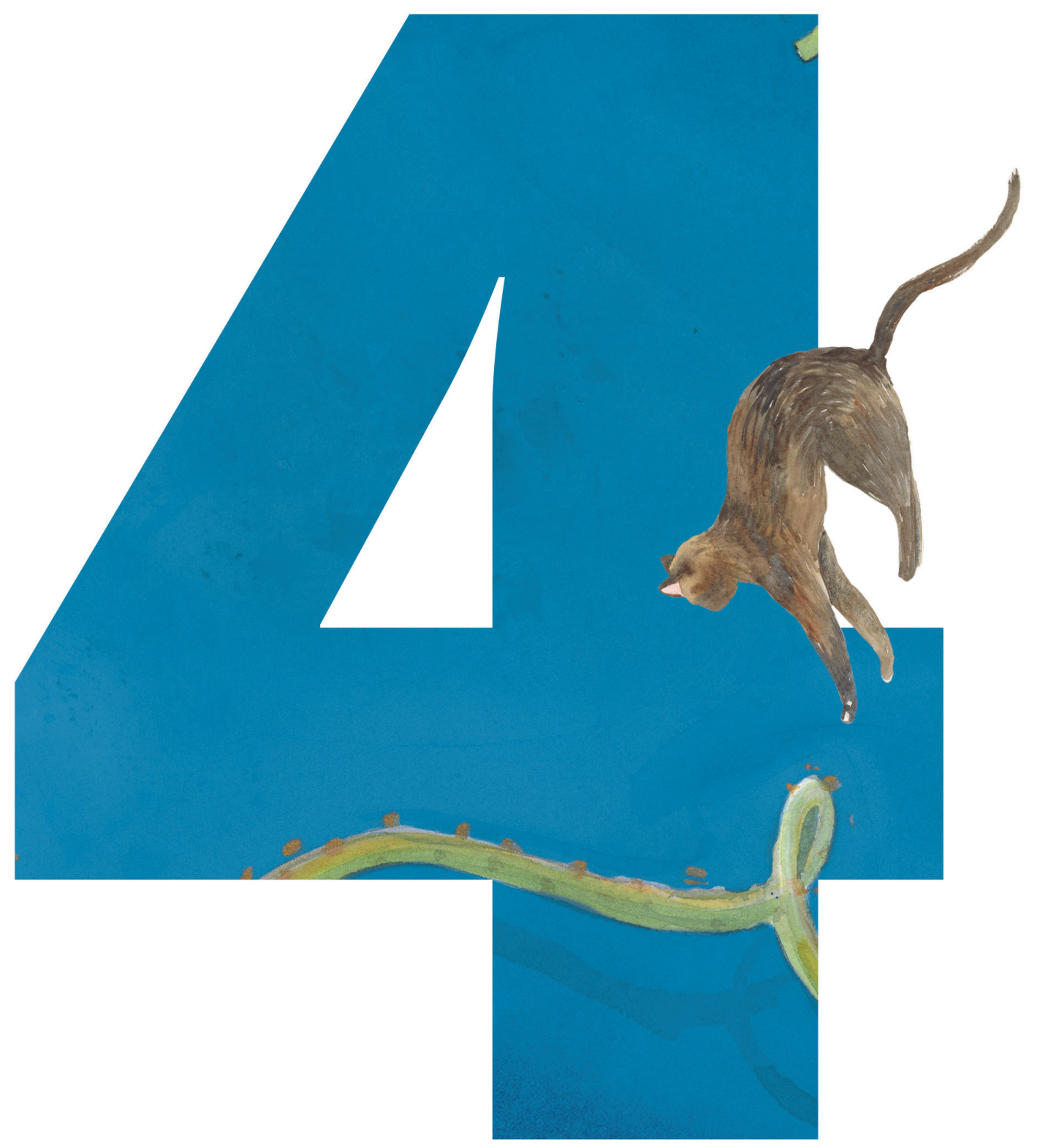




\section{Chapter 4}

Tryptophan supplementation increases the production of microbial-derived AhR agonists in an in vitro simulator of intestinal microbial ecosystem

Jonna EB Koper ${ }^{1,2}$, Antonio Dario Troise ${ }^{3}$, Linda MP Loonen², Edoardo Capuano ${ }^{1}$, Paola Vitaglione $^{3}$, Vincenzo Fogliano ${ }^{1}$ and Jerry M Wells ${ }^{2}$

\footnotetext{
1'Wageningen University, Department of Agrotechnology \& Food Sciences, The Netherlands

${ }^{2}$ Wageningen University, Department of Animal Sciences, The Netherlands

${ }^{3}$ Department of Agricultural Sciences, University of Naples "Federico II", Italy
} 


\section{Abstract}

The gut microbiome has been shown to influence metabolic pathways in mice and humans. The Aryl hydrocarbon Receptor (AhR) plays an important role in intestinal homeostasis and some microbial metabolites of tryptophan are known AhR agonists. The aim of this study was to assess the impact of tryptophan supplementation on the formation of tryptophan metabolites, AhR activation and microbiota composition in the Simulator of the Human Intestinal Microbial Ecosystem (SHIME). AhR activation, microbial composition, and tryptophan metabolites were compared during high tryptophan supplementation (4 g/L tryptophan), control and washout periods. During tryptophan supplementation, the concentration of several tryptophan metabolites was increased compared to the control and wash-out period, but AhR activation by fermenter supernatant was significantly decreased. This was due to the higher levels of tryptophan, which was found to be an antagonist of AhR signalling. Tryptophan supplementation induced most microbial changes in the transverse colon including increased relative abundance of Lactobacillus, which are known to produce AhR agonists from the metabolism of tryptophan. We conclude that tryptophan supplementation leads to increased formation of AhR agonists in the colon. Although tryptophan itself was found to be an antagonist of AhR activation, its impact on AhR activation in vivo is unknown due to uptake and transport by intestinal epithelial cells.

Keywords: Aryl hydrocarbon Receptor, SHIME, Microbiota, Tryptophan, Tryptophan metabolites 


\section{Introduction}

Species of the human gut microbiota produce numerous metabolites, including those generated by the metabolism of dietary compounds ${ }^{1,2}$. Metabolite signalling through the Aryl hydrocarbon Receptor (AhR) is an example of an interaction that can have strong effects on the immune system and intestinal homeostasis ${ }^{3-5}$. AhR is expressed in many different cell types in the body and is involved in many complex physiological processes, in particular the regulation of xenobiotic metabolising enzymes ${ }^{4,6-8}$.

AhR agonists can be found as such in the diet (e.g. certain polyphenols), they can be generated during digestion in the small intestine from dietary precursors (e.g. 3,3'-diindolylmethane from glucobrassicin) or they can be generated by microbial metabolism of tryptophan by specific species ${ }^{9-11}$. Tryptophan (Trp) is abundant in protein-rich foods like beans and nuts, cheese, meat, fish, and eggs ${ }^{12}$. The daily intake of Trp in the average 'Western' diet is approximately 600-900 $\mathrm{mg}^{13}$, of which approximately $30 \%$ is used for protein synthesis ${ }^{13,14}$. Trp supplementation is used to treat sleep disorders and other neurological disorders linked to reduced secretion of serotonin because it is a precursor in the serotonin and melatonin pathways ${ }^{15}$. Trp is also metabolised by endogenous enzymes indoleamine 2,3-dioxygenase (IDO) and L-tryptophan 2,3-dioxygenase (TDO) in the kynurenine pathway of the host, or metabolised by the gut microbiota ${ }^{9,16-20}$ to produce agonists of AhR. The metabolism of Trp to kynurenine and other AhR agonists or antagonists by microbiota is therefore of considerable interest in relation to host immune regulation. Kynurenine metabolites promote immunological tolerance through their activity on dendritic cells, T-cell anergy and apoptosis, and proliferation of Treg and Th17 cells ${ }^{21}$. Microenvironmental factors regulate the kynurenine pathway to maintain immune homeostasis.

AhR agonists produced by the microbiota have been shown to be crucial for antifungal immunity in mouse infection models?. IBD patients with polymorphisms in CARD9, leading to loss of function, have an altered microbiota that is deficient in producing AhR agonists, contributing to dysfunctional intestinal homeostasis ${ }^{10}$. In turn, microbiota composition and metabolism are affected by Trp availability in the diet. It leads to an increase of Lactobacilli, mainly L. reuteri, which subsequently affects the local immune response through the production of indole-3-aldehyde from Trp?. Given the mounting evidence connecting gut Trp metabolism to health, there is interest in supporting human health through dietary supplementation with Trp. However, the effects of such supplementation on Trp metabolism and the production of different AhR agonists in the human intestine are currently yet unknown. 
The aims of this study were to assess the impact of Trp supplementation on the formation of Trp metabolites by the human microbiota using the Simulator of the Human Intestinal Microbial Ecosystem (SHIME), and to assess the activity of the individual and pooled metabolites on the AhR signalling pathway. 


\section{Material and Methods}

\subsection{Chemicals}

All chemicals were purchased from Sigma-Aldrich (USA) unless stated otherwise. Trp metabolites screened for AhR activation were tryptamine, indole-3-aldehyde, anthranilic acid, kynurenic acid, indole-3-propionic acid, 3-hydroxy-anthranilic acid, oxindole, indole, L-kynurenine and L-Trp in concentrations ranging from $0.1-100 \mu \mathrm{M}$.

\subsection{AhR activation}

To measure the AhR activation, Luciferin transfected Dr. Chemical Activated Luciferase gene eXpression (CALUX) reporter cells (BioDetection Systems, The Netherlands, mycoplasma free) were used as previously described ${ }^{23}$. In brief, cells were grown in a-MEM growth medium (Gibco, USA) with 10\% heat-inactivated fetal calf serum (Gibco) and 1\% penicillin/streptomycin (Gibco). The cells were grown in 96-wells white clear-bottom plates (Corning, USA) at a concentration of $7.5 \times 10^{4}$ cells per well for 24 hours before stimulation. The cells were stimulated for 24 hours in triplicate with Trp and its derivatives dissolved in DMSO (Merck KGaA, Germany) or with 20 \% final volume of microbial supernatant. After stimulation, the cells were lysed with $20 \mu \mathrm{L}$ lysis buffer (Promega, USA) per well and $100 \mu \mathrm{L}$ luciferase assay buffer was added (Promega). The luminescence was measured using a Spectramax M5 (Molecular Devices, USA) immediately after the addition of the assay buffer. The results were expressed as a percentage of the positive control, $5 \mu \mathrm{M} \beta$-naphthoflavone.

\subsection{In vitro human fermentation}

The SHIME was used to simulate the human intestinal tract, as previously described ${ }^{23}$. The TWINSHIME set-up was used where two faecal samples, each from an independent donor, were used to inoculate two sets of 4 vessels in parallel. Each set of vessels simulated a combined stomach and small intestine, followed by an ascending (AC, pH 5.6 - 5.9), transverse (TC, pH 6.15 - 6.4) and descending (DC, pH 6.6 - 6.9) colon part for each donor. The donors of the faecal sample were healthy and did not take antibiotics or prebiotics for 6 and 3 months before donation, respectively. Every 8 hours, a new feeding cycle was started with a stomach phase, where $140 \mathrm{~mL}$ feed with pH 2 (1.2 g/L arabinogalactan, $2 \mathrm{~g} / \mathrm{L}$ pectin, $0.5 \mathrm{~g} / \mathrm{L}$ xylan, $0.4 \mathrm{~g} / \mathrm{L}$ glucose, $3 \mathrm{~g} / \mathrm{L}$ yeast extract, $1 \mathrm{~g} / \mathrm{L}$ special peptone, $3 \mathrm{~g} / \mathrm{L}$ mucin, $0.5 \mathrm{~g} / \mathrm{L} \mathrm{L}$-cysteine- $\mathrm{HCl}$ and $4 \mathrm{~g} / \mathrm{L}$ starch; $\mathrm{pH} 1.8$ - 2.2) was incubated for 1.5 hours, after which $60 \mathrm{~mL}$ of pancreatic juice was added (12.5 g/L NaHCO${ }_{3}, 6 \mathrm{~g} / \mathrm{L} \mathrm{Oxgall,}$ $0.9 \mathrm{~g} / \mathrm{L}$ pancreatin; $\mathrm{pH}$ 7). After a further 1.5 hours, the feed was transferred to the AC, TC and DC connected in series. The experimental design consisted of 3 weeks, including a 1-week control period with the standard feed, a 1-week Trp supplementation period where $4 \mathrm{~g} / \mathrm{L}$ L-tryptophan was added to the feed and a 1-week wash-out period where the standard feed was provided again. Fermented samples were taken every day and immediately centrifuged at $9000 \mathrm{~g}$ at $4{ }^{\circ} \mathrm{C}$, after which the supernatant was filtered using a $0.2 \mu \mathrm{m}$ RC filter (Phenomenex, Torrance, CA). The samples were stored at $-20^{\circ} \mathrm{C}$ until further analysis. 


\section{$2.4 \quad$ SCFA analysis}

Gas chromatography coupled with a flame-ionization detector (GC-FID, Shimadzu, Kyoto, Japan) was used to determine short-chain fatty acid (SCFA) composition in each sample. The samples and calibration standards were mixed in a ratio of 2:1 with an internal standard containing 0.45 $\mathrm{mg} / \mathrm{mL}$ 2-ethylbutyric acid in $0.3 \mathrm{M} \mathrm{HCl}$ and $0.9 \mathrm{M}$ oxalic acid. Subsequently, the solutions were centrifuged for $4 \mathrm{~min}$ at $20000 \mathrm{~g} .1 \mu \mathrm{L}$ of the supernatant was injected spitless in a Restek Stabilwax-DA column (30 m x $0.32 \mathrm{~mm} \times 1 \mu \mathrm{m}, \mathrm{T}_{\max } 240^{\circ} \mathrm{C}$, Restek, USA). Nitrogen was used as carrier gas, with a flow rate of $2.51 \mathrm{~mL} / \mathrm{min}$. The makeup gasses were nitrogen, hydrogen, and air with respective flow rates of $40 \mathrm{~mL} / \mathrm{min}, 30 \mathrm{~mL} / \mathrm{min}$ and $400 \mathrm{~mL} / \mathrm{min}$. The temperature was initially held at $100^{\circ} \mathrm{C}$. After injection, the temperature was increased first to $180^{\circ} \mathrm{C}$ and then to $240^{\circ} \mathrm{C}$ and both temperatures were held for $2 \mathrm{~min}$. The samples were compared to 6 calibration standard solutions containing acetic acid, propionic acid, butyric acid, valeric acid, isovaleric acid, and isobutyric acid. The results were processed using Chromeleon Edition 7 (Thermo Scientific, San Jose, CA).

\subsection{Tryptophan metabolites}

Tryptophan metabolites were analysed according to Koper et al., ${ }^{23}$. Samples were centrifuged ( $21700 \mathrm{~g}, 10 \mathrm{~min}, 4^{\circ} \mathrm{C}$ ) and diluted in $0.1 \%$ formic acid, followed by filtration using a $0.22 \mu \mathrm{m}$ cellulose filter (Phenomenex) and high-resolution mass spectrometry (HRMS) analysis. A silica modified Luna Polar C18 column (50 x $2.1 \mathrm{~mm}, 1.6 \mu \mathrm{m}$, Phenomenex) was used for the chromatographic separation of Trp and tryptophan metabolites. The mobile phases consisted of water $(\mathrm{A})$ and acetonitrile (B) both with $0.1 \% \mathrm{v} / \mathrm{v}$ of formic acid and the following gradient (min/\%B) was used: (0/2), (0.50/2), (9.5/70), (12/70). The flow rate was $200 \mu \mathrm{L} / \mathrm{min}$, the column temperature was $40^{\circ} \mathrm{C}$ and $5 \mu \mathrm{L}$ was injected. The U-HPLC system (Accela 1250, Thermo Fisher, Bremen, Germany) was interfaced to an Exactive Orbitrap HRMS (Thermo) and the analytes were detected through a heated electrospray interface (HESI-II) in positive mode by scanning the ions listed in Table S1 in the $\mathrm{m} / \mathrm{z}$ range of 50-400. Analytical performances, mass spectrometry optimization and linearity range were monitored according to Koper et al., ${ }^{23}$. Each sample was analysed in duplicate and the concentrations are given in $\mu \mathrm{M}$.

\subsection{Microbial analysis}

DNA extraction of the microbial pellets was performed using the QIAmp PowerFecal DNA Kit (Qiagen, USA). 16S ribosomal RNA sequencing of the V3-V4 region was performed by Novogene (Hong Kong). The results were analysed using the CLC bio genomics workbench (Qiagen, The Netherlands), Microbial Genomics Toolbox with the SILVA 16S v132 99\% as reference database. Further analysis and statistics were performed using the online MicrobiomeAnalyst tool (www.microbiomeanalys.ca), with filtering steps: minimal count 4, prevalence $10 \%$ and removal of $2 \%$ standard deviation. The Ward clustering analysis was shown as a heat map using Euclidean distance measurement at family level. 


\subsection{Statistical analysis}

The statistical analyses were performed using GraphPad Prism 5 (La Jolla, USA). Results are shown as mean \pm SEM. ${ }^{*} p<0.05,{ }^{* *} p<0.01$, and ${ }^{* * *} p<0.001$ were considered statistical differences. AhR activation was tested using one-way ANOVA followed by a Tukey post hoc analysis for the tryptophan derivatives and a repeated-measures ANOVA followed by a Tukey post hoc analysis for the fermentation samples. Different letters above bars represent statistically significant differences between responses to each concentration. 


\section{Results and Discussion}

\subsection{AhR activation during colonic fermentation}

To study the effects of a high Trp diet in SHIME, 4 g/L L-tryptophan, corresponding to 560 mg per feeding, was added to the standard SHIME feed for 1 week in all feeding cycles. The amount of Trp was based on dietary supplementation in human intervention studies, taking into account absorption in the small intestine ${ }^{22}$. Each day, one sample was collected from each SHIME vessel just prior to the new feeding cycle. In the control period (i.e. 1-week before the Trp supplementation period), AhR activity of the samples remained constant in each part of the colon (Figure 1). The highest AhR activation was measured in the DC, followed by the TC and AC, which is in line with previous findings ${ }^{23}$. Surprisingly, AhR activation decreased in all parts of the colon during the 1-week of Trp supplementation, with the largest decrease in the DC (Figure 1c). Finally, after the wash-out period, the AhR activation returned to the level measured in the control period in the AC and DC samples. However, on the last day of the wash-out period AhR activity in the TC was significantly higher ( $p<0.001$ ) than in the control period (Figure 1b).
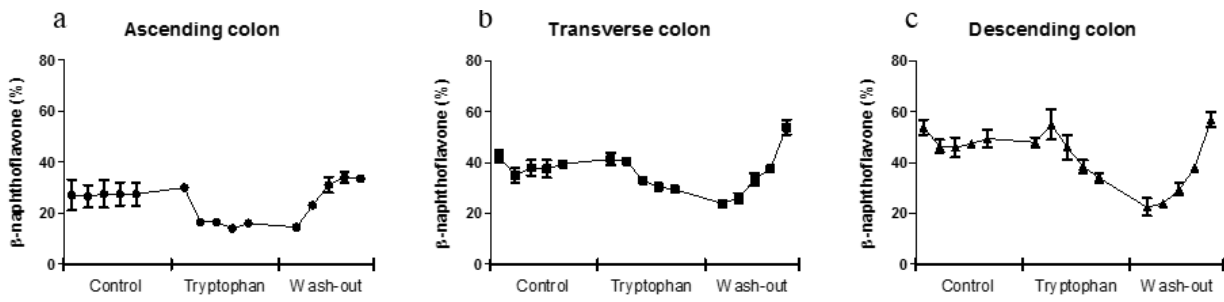

Figure 1. AhR activation of a) ascending, b) transverse and c) descending colon, where each data point represents the AhR activity at each day of the control, tryptophan supplementation and wash-out period. Measured with the CALUX reporter assay, expressed as percent of the positive control ( $\beta$-naphthoflavone, $5 \mu \mathrm{M})$ using luciferase production as readout. Data are expressed as mean of 2 donors \pm SEM.

\subsection{Tryptophan metabolites in control and supplemented diet}

Trp and its metabolites were quantified in all sample supernatants and as expected, Trp and all its metabolites increased during the Trp supplementation period (Figure 2). Trp reached a concentration of $995 \mu \mathrm{M}, 765 \mu \mathrm{M}$ and $363 \mu \mathrm{M}$ in the AC, TC, and DC, respectively. Theoretically, the concentration of Trp in the AC after the supplementation should have been $3917 \mu \mathrm{M}$, meaning that approximately $75 \%$ of the Trp was metabolised by the microbiota. Trp supplementation increased the combined amount of Trp metabolites in AC, TC, and DC compared to the control (Figure 3). From Figure 3 it is also evident that Trp supplementation increased the conversion of Trp into its metabolites especially in AC and TC but to a lesser extent in the DC. The largest increase of Trp metabolites was measured in the TC ( $<0.0001)$, indicating the importance of the TC for Trp metabolism. After the wash out period the total amount of Trp metabolites 
measured in the AC decreased to $0.7 \mu \mathrm{M}$, which is similar to the concentration present in the control period before Trp supplementation $(0.5 \mu \mathrm{M})$. The same holds true for the concentration of Trp metabolites formed in the TC after the wash-out period indicating that the production of Trp metabolites by the microbiota is directly related to the amount of available Trp.

The main metabolite formed during the Trp supplementation period was the AhR ligand oxindole, which was at highest concentration ( $34 \mu \mathrm{M}$ ) in the TC, had fluctuating concentrations (3.5 to $25 \mu \mathrm{M}$ ) in the DC and was absent in the AC (Figure 2). The second most abundant metabolite formed was 3-methylindole, with highest concentrations of $17 \mu \mathrm{M}$ in the AC, $14 \mu \mathrm{M}$ in the TC and $2 \mu \mathrm{M}$ in the DC. At the end of the wash-out period, Trp and most of its metabolites decreased to similar concentrations measured before Trp supplementation (control period in Figure 2).

The concentrations of some metabolites, namely tryptophol, indole-3-acetic acid, indole-3acetaldehyde, and tryptamine were higher on the last day of the wash-out period than in the control period. Indole and anthranilic acid had higher concentrations during and after the washout in both the TC and DC compared to the beginning of the wash-out period.

As can be seen in Figure 2, we measured a larger increase of 3-methylindole, oxindole and indole than the other Trp metabolites during Trp supplementation. This may be due to a higher rate of conversion for the reactions catalysed by tryptophanase and pyruvate amino transferase compared to those catalysed by arylformidase and transglutaminase, which are required for formation of kynurenine and xanthurenic acid ${ }^{24,25}$. Alternatively, this may be due to the presence of bacterial species with different pathways for metabolism of Trp.

Of the metabolites formed (Figure 2), tryptamine, kynurenine, indole-3-acetaldehyde, indole3-acetic acid and indole-3-aldehyde, indole, 3-methylindole, and oxindole have been reported as AhR ligands 9,26 . Several Trp metabolites produced during colonic fermentation in the SHIME system (tryptamine, indole-3-aldehyde, anthranilic acid, kynurenic acid, indole-3-propionic acid, 3-hydroxy-anthranilic acid, oxindole, indole, L-kynurenine, and L-tryptophan) were screened for their capacity to activate AhR. Of these, only tryptamine induced a relatively high dosedependent AhR activation (approx. $28 \%$ of the positive control) after stimulation with 80 and $100 \mu \mathrm{M}$ (Figure 4a). L-kynurenine, oxindole and indole induced significant but low levels of AhR activation (Figure 4b-d). Trp itself did not activate AhR in the range between 1 and $1000 \mu \mathrm{M}$. This is in line with other reports of AhR activation by Trp and its metabolites ${ }^{27,28}$. 

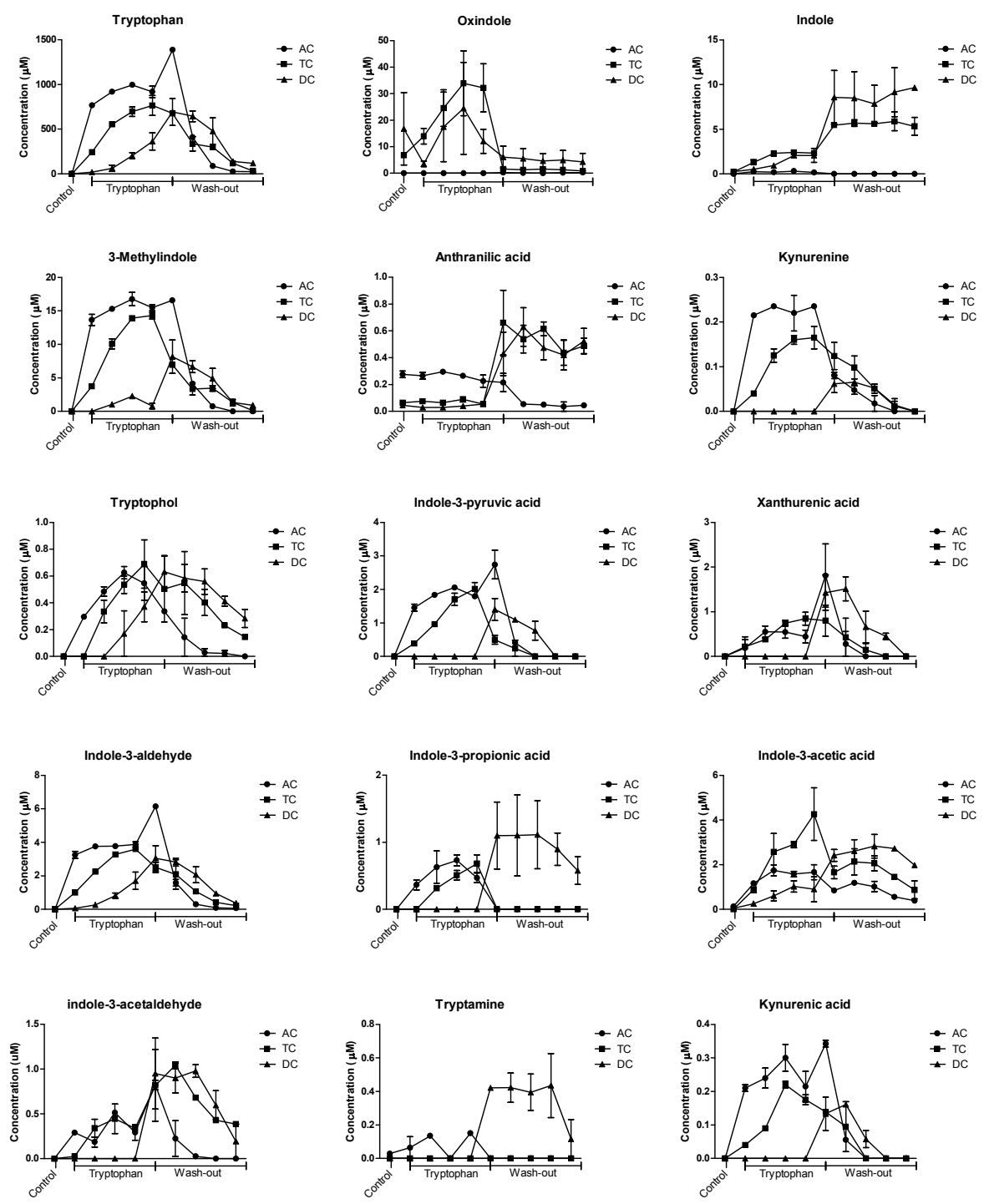

Figure 2. Evolution of Tryptophan and tryptophan metabolites concentration during control, tryptophan supplementation and wash-out period, in the ascending (AC), transverse (TC) and descending colon (DC) during in vitro SHIME fermentation. $n=2$ donors. Each data point represents the concentration at each day of the Trp supplementation and wash-out period. Only the last day of the control period is reported (first data point in the graph). 


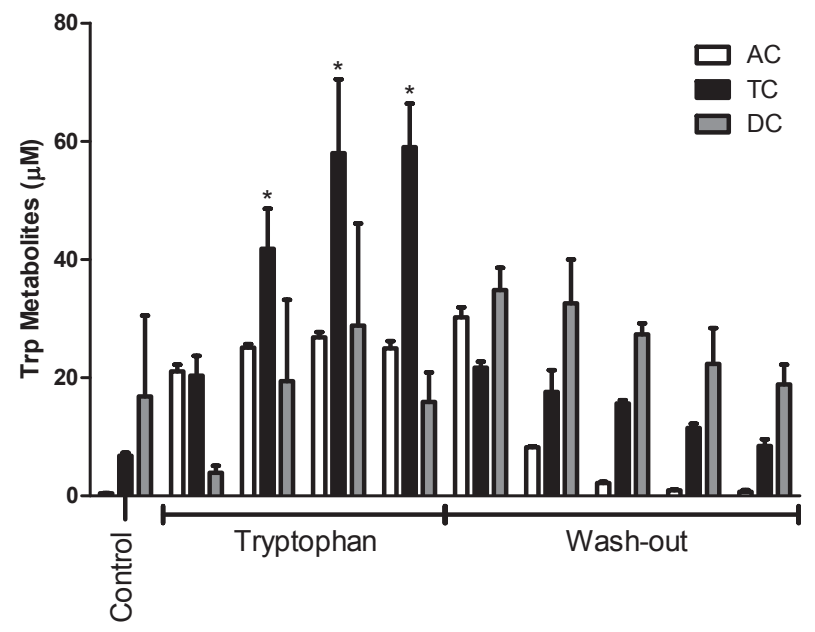

Figure 3. Cumulative concentration of tryptophan metabolites quantified using Orbitrap during control, tryptophan supplementation and wash-out period, in the ascending (AC), transverse (TC) and descending colon (DC) during in vitroSHIME fermentation. $n=2$ donors. Each data point represents the concentration at each day of the Trp supplementation and wash-out period. Only the last day of the control period is reported (first data point in the graph). ${ }^{*} p<0.05$.
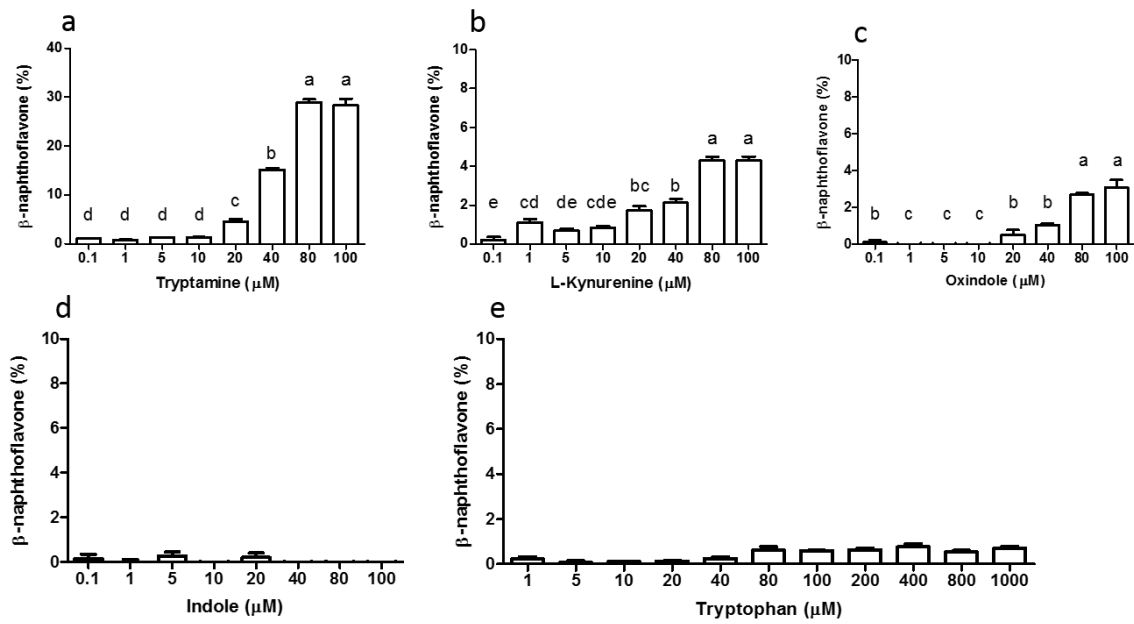

Figure 4. AhR activation of a) tryptamine, b) L-kynurenine, c) oxindole, d) indole and e) tryptophan, measured with the CALUX reporter assay, expressed as percent of the positive control ( $\beta$-naphthoflavone, $5 \mu \mathrm{M})$ using luciferase production as readout. Data are expressed as mean of 3 replicates \pm SEM, and different letters above the bar represent statistically significant different responses compared to each concentration. 
The AhR ligand indole-3-acetaldehyde ${ }^{29}$ had a higher concentration in the TC after the washout period than in the control and Trp supplementation period (Figure 2). This change may partly explain the higher AhR activity in the TC after the wash-out period (Figure 1b). This may involve the interconversion between tryptophol (which is not an AhR agonist) and indole-3acetaldehyde $^{27}$. Again, this implies a relevant role of the TC regarding the production of AhR ligands that are formed further down the Trp metabolism pathway.

Liang et al. studied supplementation of Trp in pigs and although they did not discriminate between different colon parts, they found different levels of Trp metabolites in colonic

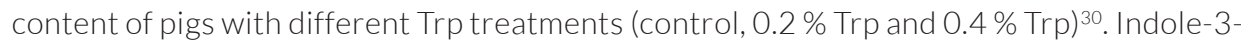
acetic acid was increased the most in the cecal content after Trp supplementation but no increase was measured in the colonic content. In our study, Trp supplementation elevated the amount of Trp available to microbiota in the AC, TC and DC by 46, 31 and 21 times respectively, even though the total amount of Trp metabolites was not proportionally higher compared to control (Figure 3).
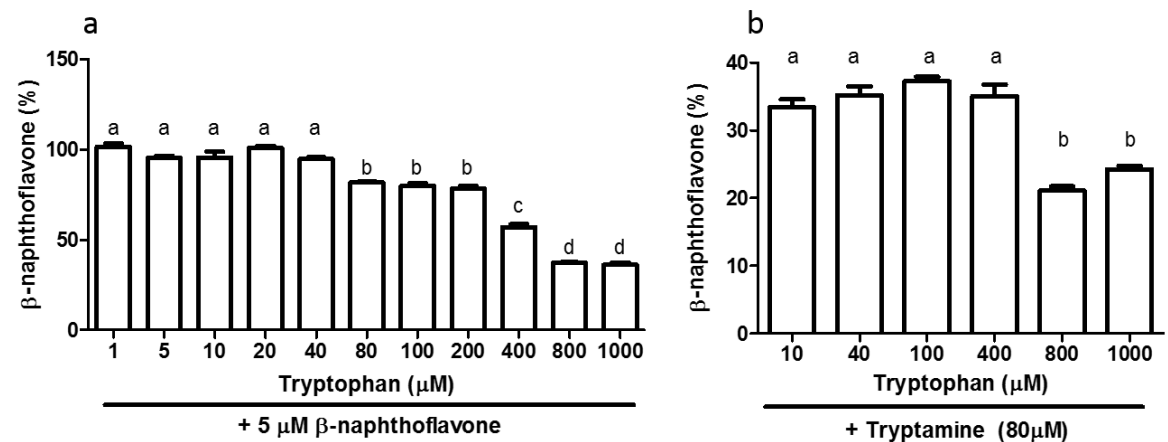

Figure 5. AhR activation measured with the CALUX reporter assay, expressed as percent of the positive control ( $\beta$-naphthoflavone, $5 \mu \mathrm{M}$ ) using luciferase production as readout. With a) different concentrations of tryptophan combined with $5 \mu \mathrm{M} \beta$-naphthoflavone and b) different concentrations of tryptophan combined with $80 \mu \mathrm{M}$ tryptamine. $n=3$. Data are expressed as mean \pm SEM, and different letters above the bar represent statistically significant different responses compared to each concentration.

To explain the fact that AhR activity was lower than the control during Trp supplementation (Figure 4), we hypothesized that Trp itself might have an antagonistic effect on AhR signalling. Indeed, we found that above concentrations of $200 \mu \mathrm{M}$, Trp inhibits AhR activation by $\beta$-naphthoflavone (Figure 5a) and tryptamine (Figure 5b), one of the most potent microbialderived AhR agonists. In the AC, Trp supplementation increased concentrations to above $200 \mu \mathrm{M}$ which would account for the decreased AhR activity in AC compared to control. This is, as far as we know, the first time Trp has been reported to antagonise AhR signalling by other 
ligands. In vivo, the antagonist effect of Trp on AhR activation in mucosal tissues may also be relevant but it is difficult to predict because Trp is transported across the epithelium by amino acid transporters ${ }^{31}$ and the concentrations found in intestinal mucosa have to our knowledge not been assessed.

\subsection{Effects of Trp supplementation on microbial composition and SCFA production}

SCFAs and the microbial composition were analysed in order to determine whether there was a change in microbial composition and fermentation leading to an altered production of SCFA. Figure 6 shows that the total production of SCFA was highest in the DC and lowest in the AC, which is in accordance with Van den Abbeele et al. ${ }^{32}$. Overall, the SCFAs concentrations in all parts of the colon remained at similar levels independently of Trp supplementation. This is in accordance with the findings of Liang et al., who did not find any differences in SCFA production with different amounts of Trp supplementation in vivo ${ }^{30}$. Also, Van den Abbeele et al., showed that the SHIME microbiota is stable over time without changed fermentation conditions like SCFAs ${ }^{32}$. Recently, it was shown that butyrate can activate the AhR pathway in vitro, in a dosedependent manner at concentrations above $1 \mathrm{mM}^{33}$. Although the butyrate concentrations in our AhR activation assay reached $<1 \mathrm{mM}$, butyrate may be important for AhR activation in vivo. However, butyrate is found in scarce amounts in the blood, suggesting its metabolism by epithelial cells, which makes its effect largely restricted to cells in the intestinal epithelium ${ }^{34,35}$.
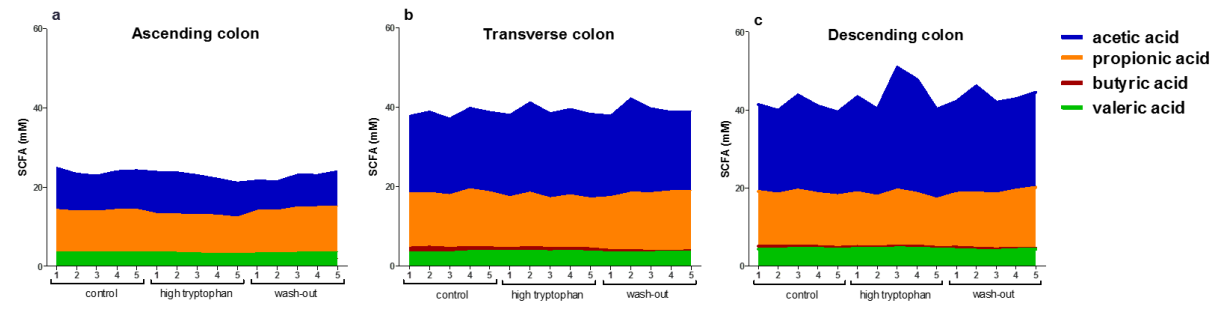

Figure 6. SCFA profile (mM) for the (a) ascending colon, (b) transverse colon and (c) descending colon, after a control, tryptophan supplementation and wash-out period of 5 days. Each data point represents the SCFA profile at each day of the control, Trp supplementation and wash-out period. $n=2$ donors.

The microbiota was compared between the donors and in the different colon compartments over the period of supplementation and wash-out. The microbiota of the two human donors was stable but differed in composition during the control period (Figure 7a, $p<0.019$ ). As anticipated, the physiological conditions in each simulated colon compartment altered the composition, independently of the donor (Figure 7b, $p<0.001$ ). No significant differences were measured in the alpha diversity during the Trp supplementation compared to the control and wash-out period (Figure 7c). 

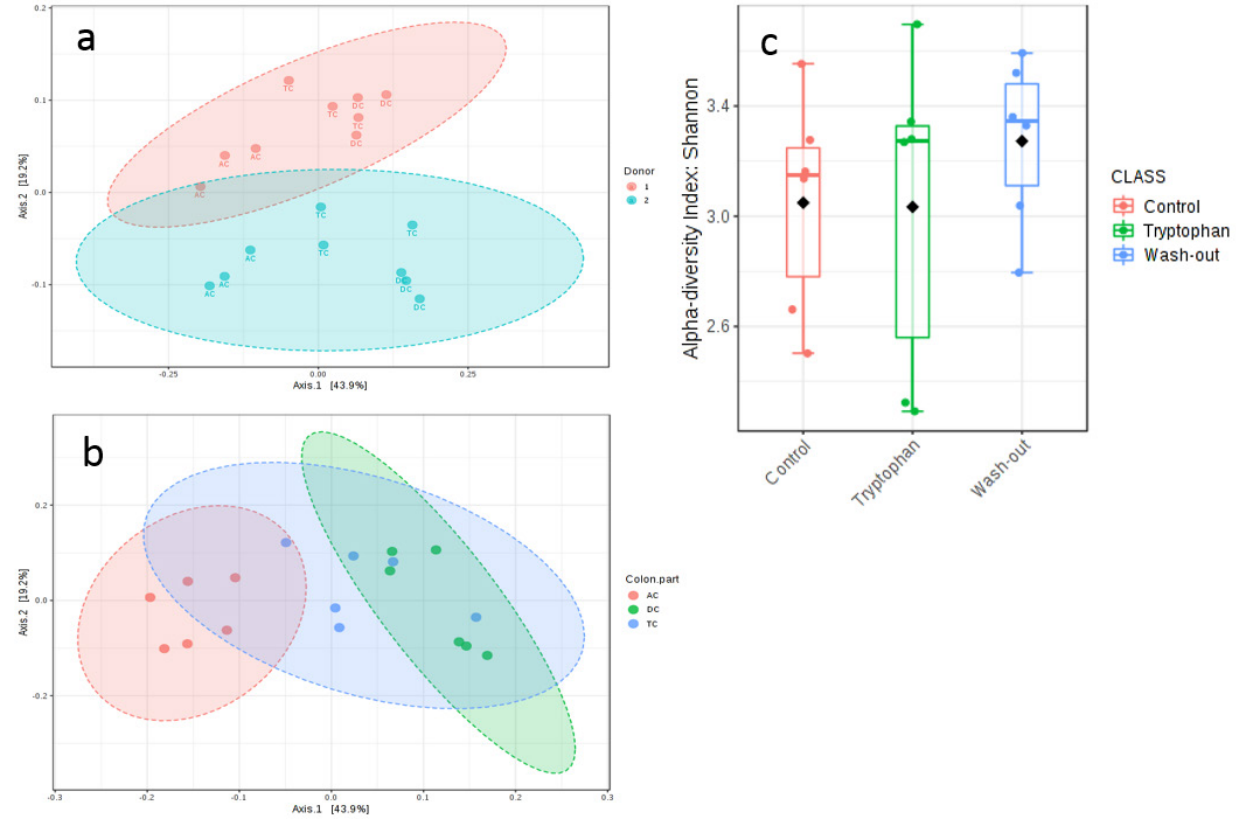

Figure 7. (a-b) Beta diversity at OTU level, using the Bray-Curtis PCOA index, showing the effects of donor (a) and colon part (b) on the overall microbial composition. (c) Alpha-diversity of the control vs tryptophan supplementation vs wash-out period at OTU level using the Shannon index.

The effect of Trp supplementation on phylum level composition was analysed for both donors in each simulated colon compartment. In the AC of donor 1 (AC1), the relative abundance of Firmicutes phylum increased from 53\% in the control period to $65 \%$ after the high Trp and $70 \%$ after the wash-out period (Figure 8). This coincided with a decreased relative abundance of the Bacteroidetes phylum, from $31 \%$ to $20 \%$ and $17 \%$ during the control, Trp supplementation and wash-out periods respectively. However, an increase in Firmicutes was not observed in AC of donor 2 (AC2) during the Trp supplementation and wash-out period. Instead the AC2 showed a small but significant decrease in abundance of Actinobacteria phylum during the Trp (16\%) and wash-out period (14\%) compared to the control (21\%).

Trp supplementation induced similar changes in the microbiota composition of the TC and DC compartments of both donors. For donor 1, the Verrucomicrobia (mainly Akkermansia) decreased during and after the Trp period (from $16 \%$ to 2 and $3 \%$ in TC 1 and from $11 \%$ to 5 and $3 \%$ in DC1). The decrease in Verrucomicrobia coincided with an increase in Bacteroidetes (from $18 \%$ to $33 \%$ ) in TC1 and in Actinobacteria in DC1 (from 7 to $13 \%$ ). In donor 2, Verrucomicrobia also decreased in the TC and DC compartments although to a lesser extent. Akkermansia muciniphyla, the only species of the phylum Verrucomicrobia is indicated to be beneficial in maintaining 
intestinal integrity ${ }^{36}$. For this reason the big decrease observed in the TC and DC of both donors (Table S2) might indicate intestinal imbalance, although our in vitro system does not represent the full intestinal complexity. Particularly it lacks the mucous layer and A. muciniphyla is a mucindegrading species. The Bacteroidetes in DC1 were highest in abundance after the Trp period (40 \%) compared to the control (32\%) and wash-out period (29\%). In donor 2, a small increase in Firmicutes was observed during the Trp supplementation in both the TC and DC (TC2 from 55 to $62 \%$ and DC2 from 41 to $51 \%)$.

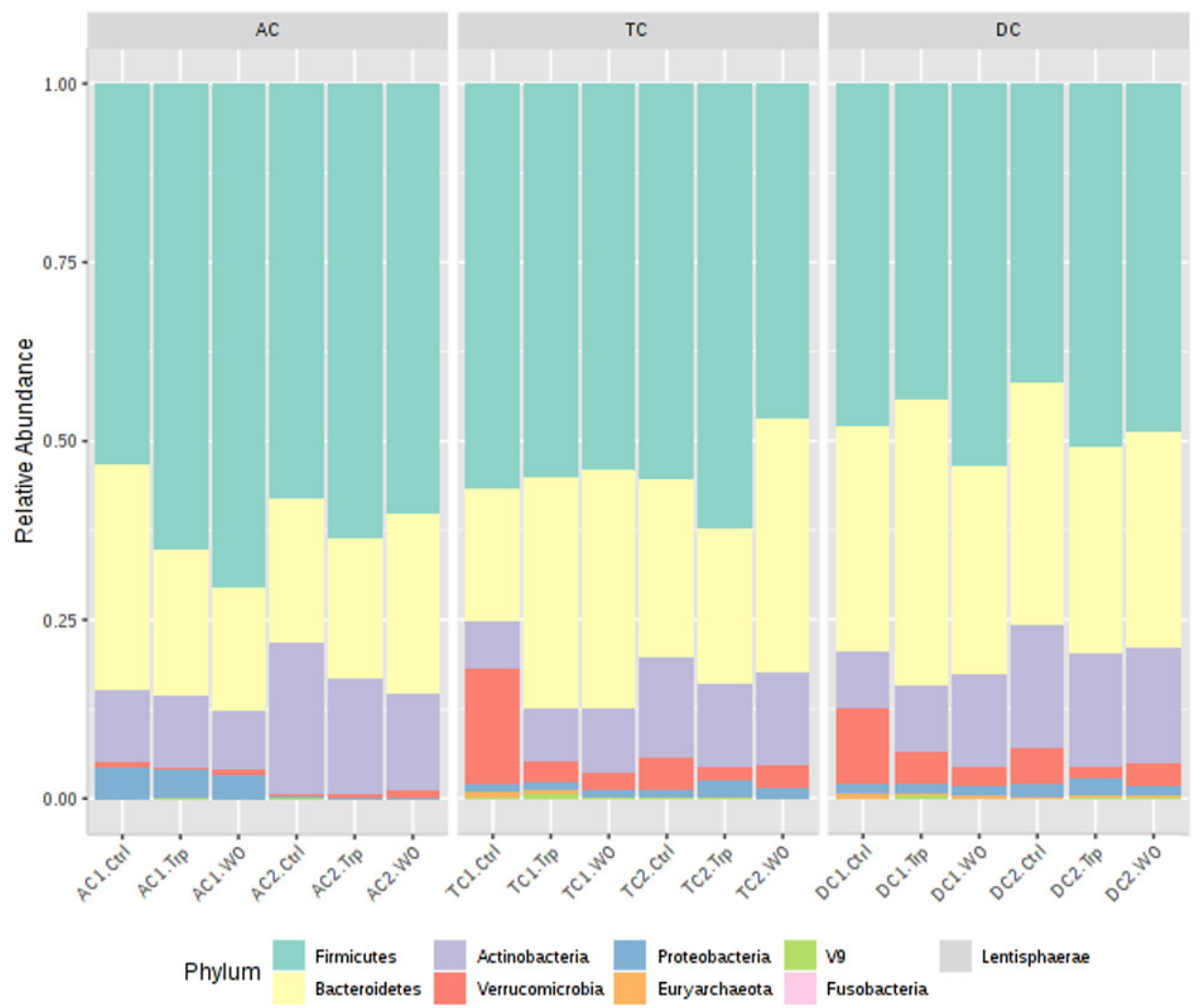

Figure 8. Relative abundance of $16 \mathrm{~S}$ rRNA sequencing at phylum level for donor 1 and donor 2 , in the ascending (AC1/2), transverse (TC1/2) and descending (DC1/2) colon at control period (Ctrl), tryptophan supplementation period (Trp) and wash-out period (WO).

The microbiota differences between donors and colon parts are shown in more in detail in a heat map cluster analysis at the family level of taxonomy for the two donors in all colon compartments after the control, Trp supplementation and wash-out period (Figure 9, Table S2). 


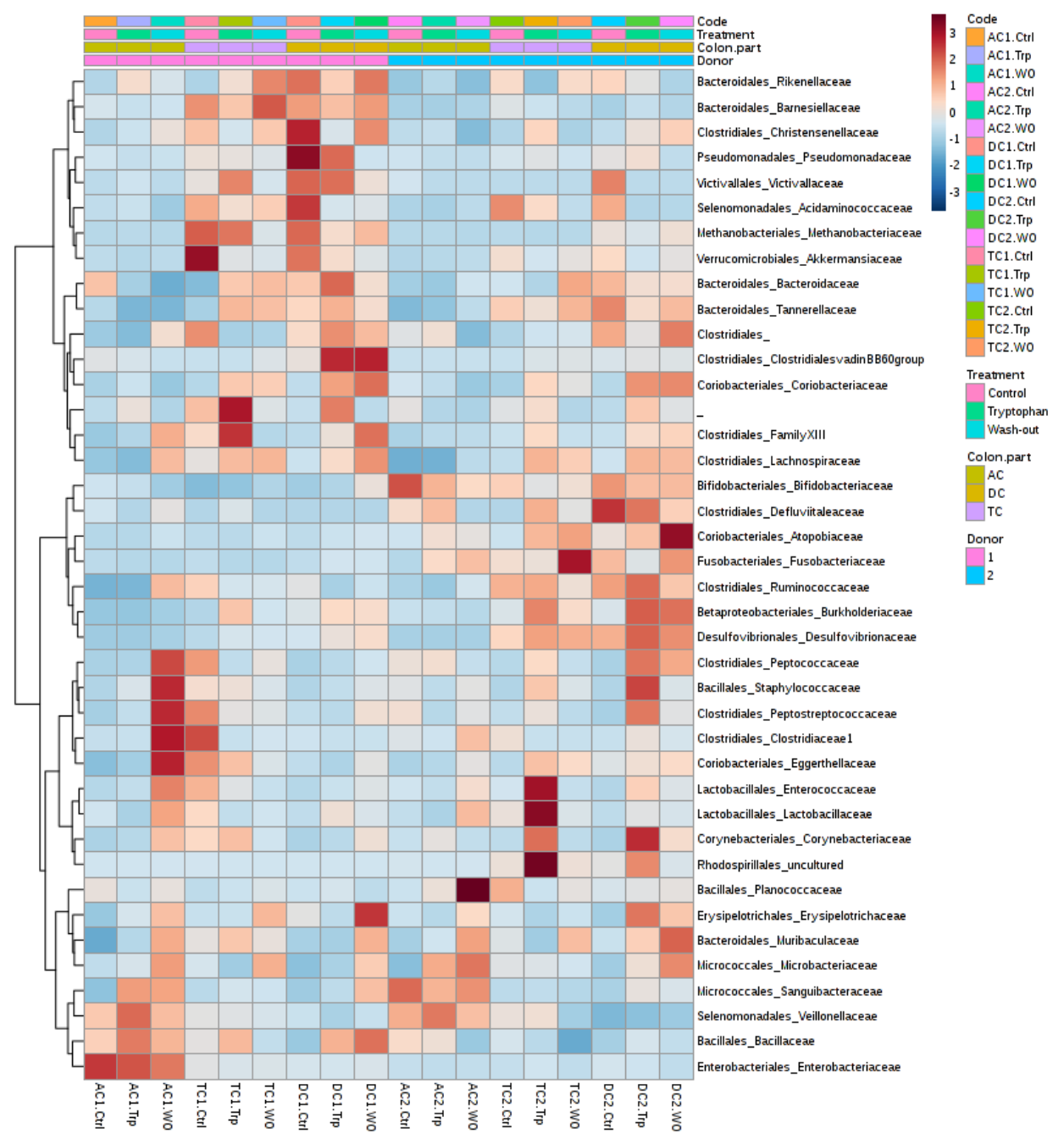

Figure 9. Heat map of the $16 \mathrm{~S}$ rRNA microbial analysis at family level of donor 1 and donor 2, ascending (AC1/2), transverse (TC1/2) and descending (DC1/2) colon at control period (Ctrl), tryptophan supplementation period (Trp) and wash-out period (WO). Red indicates a higher abundance and blue a lower abundance.

There was a higher abundance of Peptococcacea, Peptostreptococcaceae, Staphylococcaceae, Clostridiacaea, Eggerthellaceae, Enterococcaceae and Lactobacillacae in the AC of donor 1 in the wash out period. This was also observed in the AC of donor 2, but the increase in abundance of Lactobacillacae was less. Some donor-specific changes in the composition of the microbiota were also evident, for example, the large increase of Planococcaceae in the AC of donor 1 in the wash out period. Some of these families are symbiotic and produce 
bioactives with anti-inflammatory effects, for example Eggerthellacacae, which are well known to metabolize ellagitannins into urolithins ${ }^{37}$.

A major increase in Lactobacillacae and Enterococcaceae was found in TC2 after Trp supplementation. Besides, Trp supplementation increased relative abundance of Corynebacteriaceae (uncultured species) and Rhodospirillales (uncultured) in DC2. In donor 1, the abundance of these species was not significantly altered, but Trp supplementation led to an increase in several families of the Clostridiales order in both the TC and DC. There was also a decrease in Akkermansiaceae in TC1 after the Trp supplementation period compared to the control period (from 16 to $2 \%$ ), which is consistent with changes at the phylum level (Figure 8). In the TC and DC of both donors, there was a major increase in Lachnospiraceae after the Trp supplementation, which includes butyrate-producing species ${ }^{38}$ that have beneficial roles in the intestine ${ }^{39,40}$. At the end of the wash-out period, the Lachnospiraceae were still increased in abundance in the TC and DC and also increased in the AC of both donors compared to the control period. The increase in Lachnospiraceae in both donors indicates that the Trp supplementation had an effect on butyrate producers and can play a role in anti-inflammatory properties after supplementation.

Lactobacillacae and Peptococcacea are reported to be able to produce AhR ligands. Besides, an increase in Lactobacillacae in a high Trp diet is confirmed by several studies ${ }^{9,41,42}$. Further analysis of Lactobacillacae and Enterococcaceae on species level, as found in TC2 after Trp supplementation, appear to be mainly an increased abundance of Enterococcus faecalis (from 0.2 to 0.4 to $0.1 \%$ ), Lactobacillus reuteri and Lactobacillus murinus (0.02 to 0.09 to $0.02 \%$ ), which are all known to be AhR ligand producers ${ }^{42}$. The differences in microbiota after the Trp supplementation found between the two donors, combined with having a similar AhR activity, suggest that probably not only the Lactobacillacae and Peptococcacea are important when converting Trp in AhR ligands. Besides, as only relative abundance was measured, there might be differences in absolute amount of e.g. Lactobacillacae and a different amount of AhR ligands produced. Although the microbiota composition differs between donors, the AhR activation and the SCFA production were not significantly different. It can be hypothesized that different microbial species can exert similar metabolism, thereby maintaining a balanced microbial ecosystem. 


\section{Concluding remarks}

This is the first time that an intestinal model of the human microbiota ecosystem has been used to investigate the detailed profile of Trp metabolites generated in different parts of the colon and the effect of Trp supplementation on the microbial composition. Despite the fact that Trp supplementation increased the amount of Trp metabolites in the fermenter supernatants, the overall AhR activity was lower than in the control period. This was due to an antagonistic effect of Trp on AhR activation. However, Trp and its metabolites are unlikely to accumulate in the lumen of the large intestine in vivo as they do in the fermenter, and little is known on their relative transport rate across the colon epithelium. This means that the relative concentration of tryptophan compared to AhR ligands obtained thereof that we measured in our in vitro fermenter, may not fully represent the concentration ratios occurring within the colon cells.

Most Trp metabolites were produced in the TC where the largest effect of Trp on the microbiota composition was observed. In the TC, the AhR ligand producers Enterococcus faecalis, Lactobacillus reuteri and Lactobacillus murinus were increased as a result of Trp supplementation. Overall, these findings indicate that Trp supplementation can increase Trp metabolism and the production of AhR agonists.

\section{Acknowledgements}

Research presented in this publication was financially supported by the Graduate School VLAG. Erik Meulenbroeks and Wolf Tettelaar are gratefully acknowledged for their help operating the SHIME.

\section{Conflicts of interest}

The authors declare no competing interests. 


\section{References}

1 Selma, M. V., Espin, J. C. \& Tomas-Barberan, F. A. Interaction between phenolics and gut microbiota: role in human health. Journal of Agricultural and Food Chemistry 57, 6485-6501, (2009).

2 Li, M. et al. Symbiotic gut microbes modulate human metabolic phenotypes. Proceedings of the National Academy of Sciences 105, 2117-2122, (2008).

3 Kiss, E. A. et al. Natural aryl hydrocarbon receptor ligands control organogenesis of intestinal lymphoid follicles. Science 334, 1561-1565, (2011).

$4 \mathrm{Li}$, Y. et al. Exogenous stimuli maintain intraepithelial lymphocytes via aryl hydrocarbon receptor activation. Cell 147, 629-640, (2011).

5 Murray, I.A., Nichols, R. G., Zhang, L., Patterson, A. D. \& Perdew, G. H. Expression of the aryl hydrocarbon receptor contributes to the establishment of intestinal microbial community structure in mice. Scientific Reports 6, 33969, (2016).

6 Thatcher, T. H. et al. Aryl hydrocarbon receptor-deficient mice develop heightened inflammatory responses to cigarette smoke and endotoxin associated with rapid loss of the nuclear factor- $\mathrm{kB}$ component RelB. The American journal of pathology 170, 855-864, (2007).

7 Negishi, T. et al. Effects of aryl hydrocarbon receptor signaling on the modulation of TH1/TH2 balance. The Journal of Immunology 175, 7348-7356, (2005).

8 Xu, C., Li, C. Y.-T. \& Kong, A.-N. T. Induction of phase I, II and III drug metabolism/transport by xenobiotics. Archives of pharmacal research 28, 249, (2005).

9 Zelante, T. et al. Tryptophan Catabolites from Microbiota Engage Aryl Hydrocarbon Receptor and Balance Mucosal Reactivity via Interleukin-22. Immunity 39, 372-385, (2013).

10 Lamas, B. et al. CARD9 impacts colitis by altering gut microbiota metabolism of tryptophan into aryl hydrocarbon receptor ligands. Nature Medicine 22, 598-605, (2016).

11 Sun, M., Ma, N., He, T., Johnston, L. J. \& Ma, X. Tryptophan (Trp) modulates gut homeostasis via aryl hydrocarbon receptor (AhR). Critical Reviews in Food Science and Nutrition, 1-9, (2019).

12 Roager, H. M.\&Licht, T.R. Microbial tryptophan catabolites in health and disease. Nature Communications 9, 3294, (2018).

13 Allegri, G., Costa, C. V., Bertazzo, A., Biasiolo, M. \& Ragazzi, E. Enzyme activities of tryptophan metabolism along the kynurenine pathway in various species of animals. II Farmaco 58, 829-836, (2003).

14 Richard, D. M. et al. L-Tryptophan: Basic Metabolic Functions, Behavioral Research and Therapeutic Indications. International Journal of Tryptophan Research 2, 45-60, (2009).

15 Peuhkuri, K., Sihvola, N. \& Korpela, R. Diet promotes sleep duration and quality. Nutrition research 32, 309-319, (2012).

16 Opitz, C. A. et al. An endogenous tumour-promoting ligand of the human aryl hydrocarbon receptor. Nature 478, 197, (2011).

17 Wikoff, W. R. et al. Metabolomics analysis reveals large effects of gut microflora on mammalian blood metabolites. Proceedings of the National Academy of Sciences 106, 3698-3703, (2009).

18 Peters, J. Tryptophan nutrition and metabolism: an overview. Kynurenine and Serotonin pathways 345358 (1991).

19 Oxenkrug, G. F. Genetic and hormonal regulation of tryptophan-kynurenine metabolism. Annals of the New York Academy of Sciences 1122, 35-49, (2007).

20 Fujigaki, S. et al. Species Differences inl-Tryptophan-Kynurenine Pathway Metabolism: Quantification of Anthranilic Acid and Its Related Enzymes. Archives of Biochemistry and Biophysics 358, 329-335, (1998). 
21 Van der Leek, A. P., Yanishevsky, Y. \& Kozyrskyj, A. L. The kynurenine pathway as a novel link between allergy and the gut microbiome. Frontiers in immunology 8, 1374, (2017).

22 Lindseth, G., Helland, B. \& Caspers, J. The effects of dietary tryptophan on affective disorders. Archives of Psychiatric Nursing 29, 102-107, (2015).

23 Koper, J. E. B. et al. Polyphenols and Tryptophan Metabolites Activate the Aryl Hydrocarbon Receptor in an in vitro Model of Colonic Fermentation. Molecular Nutrition \& Food Research 63, 1800722, (2019).

24 Kanehisa Laboratories. (ed Kyoto Enceclopedia of Genes and Genomes (KEGG)) (2019).

25 Kanehisa, M., Furumichi, M., Tanabe, M., Sato, Y. \& Morishima, K. KEGG: new perspectives on genomes, pathways, diseases and drugs. Nucleic Acids Research 45, D353-D361, (2017).

26 Hubbard, T. D. et al. Adaptation of the human aryl hydrocarbon receptor to sense microbiota-derived indoles. Scientific Reports 5, 12689, (2015).

27 Agus, A., Planchais, J. \& Sokol, H. Gut microbiota regulation of tryptophan metabolism in health and disease. Cell Host \& Microbe 23, 716-724, (2018).

$28 \mathrm{Jin}$, U.-H. et al. Microbiome-derived tryptophan metabolites and their aryl hydrocarbon receptordependent agonist and antagonist activities. Molecular Pharmacology 85, 777-788, (2014).

29 Alexeev, E. E. et al. Microbiota-derived indole metabolites promote human and murine intestinal homeostasis through regulation of interleukin-10 receptor. The American journal of pathology $\mathbf{1 8 8}$ 1183-1194, (2018).

30 Liang, H. et al. Dietary L-Tryptophan Modulates the Structural and Functional Composition of the Intestinal Microbiome in Weaned Piglets. Frontiers in Microbiology 9, (2018).

31 Cohen, L. L. \& Huang, K. Intestinal transport of tryptophan and its derivatives. American Journal of Physiology-Legacy Content 206, 647-652, (1964).

32 Van den Abbeele, P. et al. Microbial community development in a dynamic gut model is reproducible, colon region specific, and selective for Bacteroidetes and Clostridium cluster IX. Applied and Environmental Microbiology 76, 5237-5246, (2010).

33 Marinelli, L.et al. Identification of the novel role of butyrate as AhR ligand in human intestinal epithelial cells. Scientific Reports 9 , 643, (2019).

34 Kim, C. H., Park, J. \& Kim, M. Gut microbiota-derived short-chain fatty acids, T cells, and inflammation. Immune network 14, 277-288, (2014).

35 Kim, C. H. Immune regulation by microbiome metabolites. Immunology 154, 220-229, (2018).

36 Geerlings, S., Kostopoulos, I., de Vos, W. \& Belzer, C. Akkermansia muciniphila in the human gastrointestinal tract: when, where, and how? Microorganisms 6, 75, (2018).

37 Selma, M. V. et al. Isolation of human intestinal bacteria capable of producing the bioactive metabolite isourolithin a from ellagic acid. Frontiers in Microbiology 8, 1521, (2017).

38 Qian, L., Gao, R., Huang, J. \& Qin, H. Supplementation of triple viable probiotics combined with dietary intervention is associated with gut microbial improvement in humans on a high-fat diet. Experimental and Therapeutic Medicine 18, 2262-2270, (2019).

39 Shaoul, R. \& Day, A. S. Nutritional regulators of intestinal inflammation. Current Opinion in Gastroenterology, (2019).

40 Zhang, J. et al. Beneficial effect of butyrate-producing Lachnospiraceae on stress-induced visceral hypersensitivity in rats. Journal of Gastroenterology and Hepatology, (2018).

41 Wlodarska, M. et al. Indoleacrylic acid produced by commensal peptostreptococcus species suppresses inflammation. Cell Host \& Microbe 22, 25-37. e26, (2017).

42 Lamas, B., Natividad, J. M. \& Sokol, H. Aryl hydrocarbon receptor and intestinal immunity. Mucosal Immunology 11, 1024-1038, (2018). 



\section{Supporting information}

Table S1. High resolution mass spectrometry performances, RT (retention time, min), EC (elemental composition), TM (theoretical mass, $[\mathrm{M}+\mathrm{H}]^{+}$), EM (experimental mass, $\left.[\mathrm{M}+\mathrm{H}]^{+}\right), \Delta \mathrm{ppm}$ (mass accuracy).

\begin{tabular}{lccccc}
\hline Compound Name & EC & RT & TM & TE & $\Delta$ ppm \\
\hline L-kynurenine & $\mathrm{C}_{10} \mathrm{H}_{12} \mathrm{~N}_{2} \mathrm{O}_{3}$ & 2.3 & 209.09207 & 209.09241 & 1.6 \\
Indole-3-acetaldehyde & $\mathrm{C}_{10} \mathrm{H}_{9} \mathrm{NO}$ & 3.2 & 160.07569 & 160.07533 & -2.2 \\
Kynurenic acid & $\mathrm{C}_{10} \mathrm{H}_{7} \mathrm{NO}_{3}$ & 3.8 & 190.04987 & 190.04899 & -4.6 \\
Indole-3-pyruvate & $\mathrm{C}_{11} \mathrm{H}_{9} \mathrm{NO}_{3}$ & 4.0 & 204.06552 & 204.06533 & -0.9 \\
Xanthurenic acid & $\mathrm{C}_{10} \mathrm{H}_{7} \mathrm{NO}_{4}$ & 4.1 & 206.04478 & 206.04428 & -2.4 \\
Tryptophan & $\mathrm{C}_{11} \mathrm{H}_{12} \mathrm{~N}_{2} \mathrm{O}_{2}$ & 4.2 & 205.09715 & 205.09722 & 0.3 \\
Indole-3-aldehyde & $\mathrm{C}_{9} \mathrm{H}_{7} \mathrm{NO}$ & 4.2 & 146.06004 & 146.06047 & 2.9 \\
3-methylindole & $\mathrm{C}_{9} \mathrm{H}_{9} \mathrm{~N}$ & 4.3 & 132.08078 & 132.08066 & -0.9 \\
Tryptamine & $\mathrm{C}_{10} \mathrm{H}_{12} \mathrm{~N}_{2}$ & 4.4 & 161.10732 & 161.10711 & -1.3 \\
Anthranilic acid & $\mathrm{C}_{7} \mathrm{H}_{7} \mathrm{NO}_{2}$ & 4.8 & 138.05496 & 138.05512 & 1.2 \\
Oxindole & $\mathrm{C}_{8} \mathrm{H}_{7} \mathrm{NO}$ & 5.2 & 134.06004 & 134.06044 & 3.0 \\
Tryptophol & $\mathrm{C}_{10} \mathrm{H}_{11} \mathrm{NO}$ & 5.8 & 162.09134 & 162.09151 & 1.0 \\
Indole-3-acetic acid & $\mathrm{C}_{10} \mathrm{H}_{9} \mathrm{NO}_{2}$ & 5.8 & 176.07061 & 176.07033 & -1.6 \\
Indole-3-propionic acid & $\mathrm{C}_{11} \mathrm{H}_{11} \mathrm{NO}_{2}$ & 6.3 & 190.08626 & 190.08629 & 0.2 \\
Indole & $\mathrm{C}_{8} \mathrm{H}_{7} \mathrm{~N}$ & 6.8 & 118.06513 & 118.06488 & -2.1 \\
\hline
\end{tabular}

Table S2. Relative abundance as percentage of the 16S rRNA microbial analysis at family level.

\begin{tabular}{lrrrrrrrr}
\hline Treatment & Ctrl & Trp & WO & Ctrl & Trp & WO & Ctrl & Trp \\
Colon part & AC & AC & AC & AC & AC & AC & TC & TC \\
Donor & $\mathbf{1}$ & $\mathbf{1}$ & $\mathbf{1}$ & $\mathbf{2}$ & $\mathbf{2}$ & $\mathbf{2}$ & $\mathbf{1}$ & $\mathbf{1}$ \\
\hline Acidaminococcaceae & 0.12 & 0.13 & 0.10 & 0.11 & 0.11 & 0.12 & 0.22 & 0.17 \\
Akkermansiaceae & 0.77 & 0.32 & 0.66 & 0.26 & 0.47 & 0.84 & 16.23 & 2.83 \\
Atopobiaceae & 0.00 & 0.00 & 0.00 & 0.01 & 0.01 & 0.01 & 0.00 & 0.00 \\
Bacillaceae & 0.01 & 0.01 & 0.01 & 0.01 & 0.01 & 0.00 & 0.01 & 0.01 \\
Bacteroidaceae & 30.85 & 19.67 & 15.64 & 19.49 & 18.80 & 23.27 & 17.16 & 30.32 \\
Barnesiellaceae & 0.01 & 0.01 & 0.01 & 0.00 & 0.00 & 0.00 & 0.02 & 0.02 \\
Bifidobacteriaceae & 9.59 & 9.11 & 7.33 & 20.05 & 15.22 & 12.89 & 5.99 & 6.52 \\
Burkholderiaceae & 0.02 & 0.02 & 0.02 & 0.03 & 0.03 & 0.03 & 0.03 & 0.06 \\
Christensenellaceae & 0.00 & 0.01 & 0.01 & 0.00 & 0.00 & 0.00 & 0.01 & 0.01 \\
Clostridiaceae1 & 0.01 & 0.02 & 1.59 & 0.16 & 0.06 & 0.65 & 1.31 & 0.02 \\
ClostridialesvadinBB60 & 0.00 & 0.00 & 0.00 & 0.00 & 0.00 & 0.00 & 0.00 & 0.00
\end{tabular}




\begin{tabular}{rrrrrrrrrr} 
WO & Ctrl & Trp & WO & Ctrl & Trp & WO & Ctrl & Trp & WO \\
TC & TC & TC & TC & DC & DC & DC & DC & DC & DC \\
$\mathbf{1}$ & $\mathbf{2}$ & $\mathbf{2}$ & $\mathbf{2}$ & $\mathbf{1}$ & $\mathbf{1}$ & $\mathbf{1}$ & $\mathbf{2}$ & $\mathbf{2}$ & $\mathbf{2}$ \\
\hline 0.19 & 0.24 & 0.18 & 0.12 & 0.29 & 0.14 & 0.14 & 0.22 & 0.12 & 0.12 \\
2.40 & 4.39 & 1.83 & 3.30 & 10.71 & 4.62 & 2.65 & 5.04 & 1.68 & 3.21 \\
0.00 & 0.01 & 0.03 & 0.03 & 0.00 & 0.00 & 0.01 & 0.01 & 0.02 & 0.06 \\
0.00 & 0.00 & 0.00 & 0.00 & 0.00 & 0.01 & 0.01 & 0.00 & 0.00 & 0.00 \\
31.44 & 23.30 & 20.63 & 33.57 & 30.34 & 38.65 & 27.39 & 32.08 & 27.11 & 27.51 \\
0.03 & 0.01 & 0.01 & 0.00 & 0.02 & 0.02 & 0.02 & 0.00 & 0.01 & 0.00 \\
8.07 & 13.54 & 10.80 & 12.02 & 7.31 & 8.12 & 11.48 & 16.81 & 14.55 & 14.87 \\
0.03 & 0.04 & 0.08 & 0.05 & 0.04 & 0.05 & 0.05 & 0.04 & 0.09 & 0.08 \\
0.01 & 0.00 & 0.01 & 0.00 & 0.02 & 0.01 & 0.02 & 0.00 & 0.01 & 0.01 \\
0.08 & 0.32 & 0.02 & 0.02 & 0.05 & 0.02 & 0.04 & 0.02 & 0.29 & 0.11 \\
0.00 & 0.00 & 0.00 & 0.00 & 0.00 & 0.01 & 0.01 & 0.00 & 0.00 & 0.00
\end{tabular}


Table S2. Continued

\begin{tabular}{|c|c|c|c|c|c|c|c|c|}
\hline Treatment & Ctrl & Trp & Wo & Ctrl & Trp & wo & Ctrl & Trp \\
\hline Colon part & $\mathrm{AC}$ & $\mathrm{AC}$ & $\mathrm{AC}$ & $\mathrm{AC}$ & $\mathrm{AC}$ & $\mathrm{AC}$ & $\mathrm{TC}$ & $\mathrm{TC}$ \\
\hline Donor & 1 & 1 & 1 & 2 & 2 & 2 & 1 & 1 \\
\hline Coriobacteriaceae & 0.41 & 0.51 & 0.35 & 0.53 & 0.48 & 0.36 & 0.44 & 0.77 \\
\hline Corynebacteriaceae & 0.00 & 0.00 & 0.00 & 0.00 & 0.00 & 0.00 & 0.00 & 0.01 \\
\hline Defluviitaleaceae & 0.00 & 0.00 & 0.00 & 0.00 & 0.00 & 0.00 & 0.00 & 0.00 \\
\hline Desulfovibrionaceae & 0.07 & 0.07 & 0.13 & 0.14 & 0.12 & 0.14 & 0.24 & 0.43 \\
\hline Eggerthellaceae & 0.01 & 0.02 & 0.12 & 0.02 & 0.02 & 0.04 & 0.09 & 0.07 \\
\hline Enterobacteriaceae & 4.34 & 3.92 & 3.32 & 0.17 & 0.12 & 0.14 & 0.77 & 0.56 \\
\hline Enterococcaceae & 0.00 & 0.00 & 0.06 & 0.01 & 0.00 & 0.02 & 0.04 & 0.02 \\
\hline Erysipelotrichaceae & 0.01 & 0.09 & 0.20 & 0.08 & 0.05 & 0.16 & 0.07 & 0.07 \\
\hline FamilyXIII, Clostridiales & 0.01 & 0.02 & 0.08 & 0.02 & 0.03 & 0.03 & 0.06 & 0.13 \\
\hline Fusobacteriaceae & 0.00 & 0.00 & 0.00 & 0.00 & 0.00 & 0.01 & 0.00 & 0.00 \\
\hline Lachnospiraceae & 5.59 & 4.88 & 12.15 & 4.26 & 4.31 & 7.10 & 9.13 & 12.12 \\
\hline Lactobacillaceae & 0.01 & 0.00 & 0.04 & 0.01 & 0.00 & 0.04 & 0.03 & 0.01 \\
\hline Methanobacteriaceae & 0.01 & 0.01 & 0.00 & 0.00 & 0.01 & 0.00 & 0.64 & 0.57 \\
\hline Microbacteriaceae & 0.00 & 0.01 & 0.01 & 0.00 & 0.01 & 0.01 & 0.01 & 0.00 \\
\hline Muribaculaceae & 0.08 & 0.42 & 1.15 & 0.34 & 0.56 & 1.21 & 0.70 & 0.99 \\
\hline Peptococcaceae & 0.00 & 0.00 & 0.02 & 0.01 & 0.01 & 0.00 & 0.02 & 0.00 \\
\hline Peptostreptococcaceae & 0.05 & 0.21 & 1.66 & 0.55 & 0.14 & 0.42 & 1.15 & 0.44 \\
\hline Planococcaceae & 0.00 & 0.00 & 0.00 & 0.00 & 0.00 & 0.01 & 0.00 & 0.00 \\
\hline Pseudomonadaceae & 0.02 & 0.01 & 0.02 & 0.02 & 0.01 & 0.01 & 0.07 & 0.06 \\
\hline Rikenellaceae & 0.02 & 0.03 & 0.03 & 0.02 & 0.02 & 0.02 & 0.02 & 0.03 \\
\hline Ruminococcaceae & 0.61 & 0.78 & 6.24 & 2.09 & 1.58 & 3.30 & 5.39 & 3.32 \\
\hline Sanguibacteraceae & 0.10 & 0.38 & 0.37 & 0.44 & 0.34 & 0.39 & 0.16 & 0.20 \\
\hline Staphylococcaceae & 0.00 & 0.00 & 0.01 & 0.00 & 0.00 & 0.00 & 0.00 & 0.00 \\
\hline Tannerellaceae & 0.52 & 0.32 & 0.32 & 0.33 & 0.38 & 0.55 & 0.46 & 1.00 \\
\hline uncultured & 0.00 & 0.00 & 0.01 & 0.02 & 0.00 & 0.01 & 0.01 & 0.00 \\
\hline unknown & 0.05 & 0.18 & 0.02 & 0.16 & 0.03 & 0.01 & 0.32 & 0.70 \\
\hline Veillonellaceae & 46.69 & 58.82 & 48.31 & 50.64 & 57.05 & 48.22 & 39.20 & 38.55 \\
\hline Victivallaceae & 0.00 & 0.00 & 0.00 & 0.00 & 0.00 & 0.00 & 0.00 & 0.00 \\
\hline
\end{tabular}




\begin{tabular}{|c|c|c|c|c|c|c|c|c|c|}
\hline Wo & Ctrl & Trp & wo & Ctrl & Trp & wo & Ctrl & Trp & wo \\
\hline TC & TC & TC & TC & DC & $\mathrm{DC}$ & $\mathrm{DC}$ & $\mathrm{DC}$ & $\mathrm{DC}$ & DC \\
\hline 1 & 2 & 2 & 2 & 1 & 1 & 1 & 2 & 2 & 2 \\
\hline 0.76 & 0.40 & 0.73 & 0.60 & 0.48 & 0.91 & 1.05 & 0.45 & 0.95 & 0.98 \\
\hline 0.00 & 0.00 & 0.01 & 0.00 & 0.00 & 0.00 & 0.00 & 0.00 & 0.01 & 0.00 \\
\hline 0.00 & 0.00 & 0.00 & 0.00 & 0.00 & 0.00 & 0.00 & 0.00 & 0.00 & 0.00 \\
\hline 0.40 & 0.79 & 1.18 & 1.10 & 0.40 & 0.61 & 0.73 & 1.09 & 1.54 & 1.29 \\
\hline 0.04 & 0.03 & 0.07 & 0.06 & 0.03 & 0.02 & 0.04 & 0.04 & 0.05 & 0.06 \\
\hline 0.59 & 0.19 & 0.36 & 0.12 & 0.49 & 0.44 & 0.58 & 0.46 & 0.34 & 0.10 \\
\hline 0.01 & 0.01 & 0.09 & 0.01 & 0.00 & 0.01 & 0.01 & 0.00 & 0.03 & 0.01 \\
\hline 0.21 & 0.09 & 0.04 & 0.08 & 0.11 & 0.06 & 0.37 & 0.02 & 0.30 & 0.19 \\
\hline 0.02 & 0.03 & 0.06 & 0.03 & 0.03 & 0.05 & 0.11 & 0.03 & 0.06 & 0.07 \\
\hline 0.00 & 0.00 & 0.00 & 0.02 & 0.00 & 0.00 & 0.00 & 0.01 & 0.00 & 0.01 \\
\hline 12.41 & 7.35 & 12.49 & 11.23 & 7.76 & 10.35 & 13.93 & 7.88 & 12.37 & 12.12 \\
\hline 0.01 & 0.02 & 0.09 & 0.02 & 0.01 & 0.02 & 0.01 & 0.01 & 0.02 & 0.01 \\
\hline 0.12 & 0.01 & 0.00 & 0.01 & 0.61 & 0.23 & 0.37 & 0.18 & 0.11 & 0.20 \\
\hline 0.01 & 0.01 & 0.01 & 0.01 & 0.00 & 0.00 & 0.01 & 0.00 & 0.01 & 0.01 \\
\hline 0.73 & 0.64 & 0.32 & 1.05 & 0.36 & 0.34 & 1.12 & 0.52 & 0.93 & 1.49 \\
\hline 0.01 & 0.00 & 0.01 & 0.00 & 0.00 & 0.00 & 0.00 & 0.00 & 0.02 & 0.02 \\
\hline 0.39 & 0.21 & 0.50 & 0.17 & 0.21 & 0.16 & 0.55 & 0.06 & 1.24 & 0.42 \\
\hline 0.00 & 0.01 & 0.00 & 0.00 & 0.00 & 0.00 & 0.00 & 0.00 & 0.00 & 0.00 \\
\hline 0.04 & 0.03 & 0.05 & 0.02 & 0.37 & 0.25 & 0.02 & 0.06 & 0.08 & 0.00 \\
\hline 0.05 & 0.03 & 0.02 & 0.03 & 0.05 & 0.04 & 0.05 & 0.03 & 0.03 & 0.02 \\
\hline 3.38 & 6.54 & 6.82 & 4.46 & 3.87 & 1.97 & 3.07 & 7.22 & 8.60 & 5.86 \\
\hline 0.19 & 0.16 & 0.17 & 0.15 & 0.12 & 0.17 & 0.33 & 0.14 & 0.23 & 0.21 \\
\hline 0.00 & 0.00 & 0.00 & 0.00 & 0.00 & 0.00 & 0.00 & 0.00 & 0.01 & 0.00 \\
\hline 0.97 & 0.90 & 0.77 & 1.01 & 0.86 & 1.02 & 0.80 & 1.17 & 0.81 & 0.98 \\
\hline 0.00 & 0.09 & 0.72 & 0.10 & 0.00 & 0.00 & 0.00 & 0.06 & 0.36 & 0.03 \\
\hline 0.14 & 0.14 & 0.22 & 0.03 & 0.07 & 0.48 & 0.05 & 0.05 & 0.29 & 0.15 \\
\hline 37.26 & 40.44 & 41.71 & 30.59 & 35.37 & 31.22 & 34.98 & 26.28 & 27.73 & 29.78 \\
\hline 0.00 & 0.00 & 0.00 & 0.00 & 0.01 & 0.00 & 0.00 & 0.00 & 0.00 & 0.00 \\
\hline
\end{tabular}




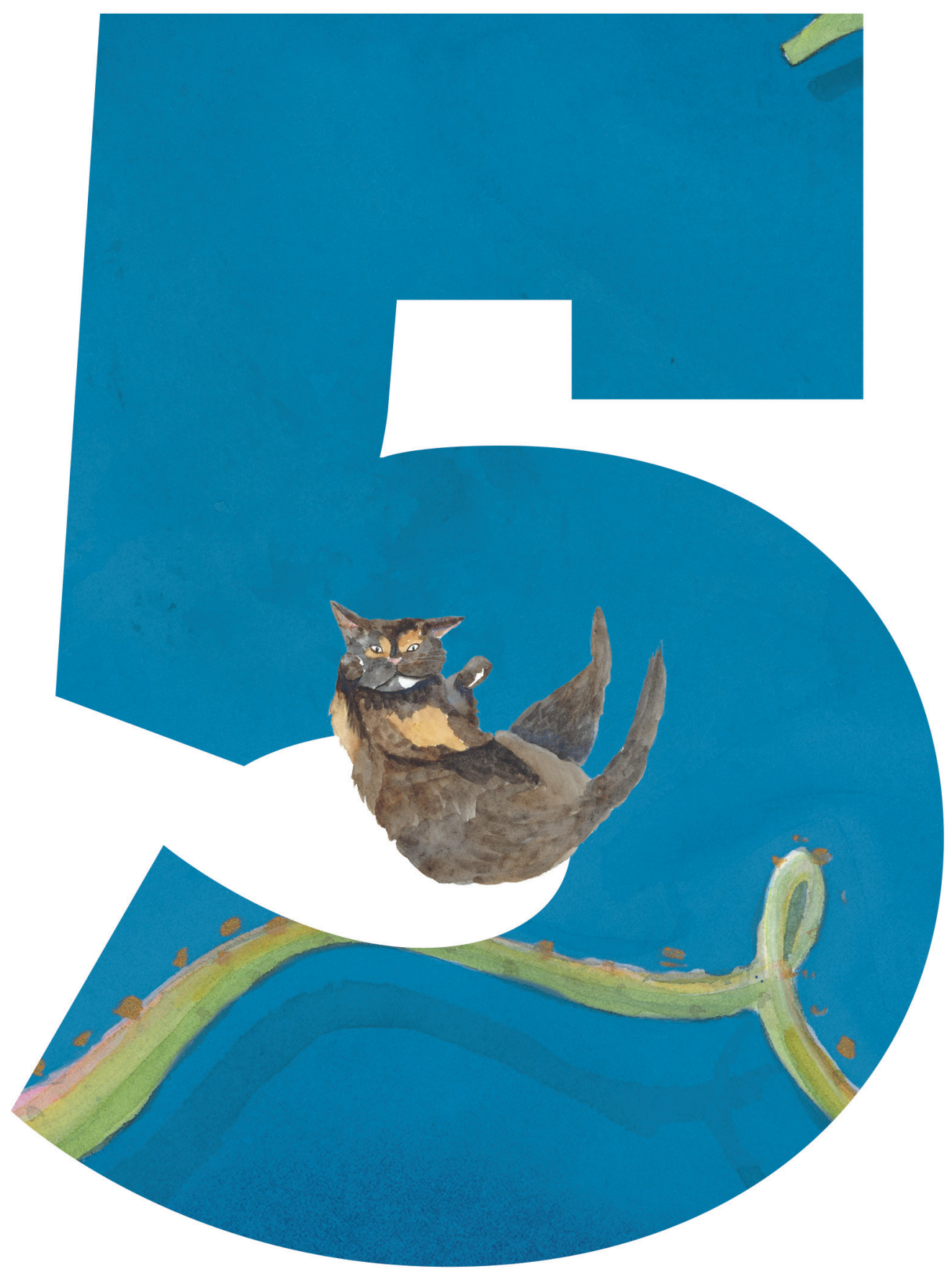




\section{Chapter 5}

\section{Aryl hydrocarbon Receptor activation during in vitro and in vivo digestion of broccoli (brassica oleracea) after different cooking methods}

Jonna EB Koper ${ }^{1,2}$, Maaike Kortekaas ${ }^{1}$, Linda MP Loonen², Zhan Huang ${ }^{1,2}$, Jerry M Wells², Chris IR Gill ${ }^{3}$, Laura Kirsty Pourshahidi ${ }^{3}$, Gordon McDougall ${ }^{4}$, Ian Rowland ${ }^{5}$, Gema PereiraCaro $^{6}$, Vincenzo Fogliano ${ }^{1}$, Edoardo Capuano ${ }^{1}$

\footnotetext{
${ }^{1}$ Wageningen University, Department of Agrotechnology \& Food Sciences, The Netherlands

2Wageningen University, Department of Animal Sciences, The Netherlands

${ }^{3}$ Ulster University, Nutrition Innovation Centre for Food and Health (NICHE), School of Biomedical Sciences, United Kingdom

${ }^{4}$ The James Hutton Institute, United Kingdom

5 University of Reading, The Hugh Sinclair Human Nutrition Unit, United Kingdom

${ }^{6}$ IFAPA-Alameda del Obispo, Department of Food Science and Health, Spain
}

Submitted for publication 


\section{Abstract}

Broccoli is rich in glucosinolates, which can be converted upon chewing and processing into various metabolites, including Aryl hydrocarbon Receptor (AhR) ligands. Activation of AhR plays an important role in overall gut homeostasis but the role of broccoli processing on the generation of AhR ligands is still largely unknown. In this study, the effects of temperature, cooking method (steaming versus boiling), gastric $\mathrm{pH}$ and further digestion of broccoli on AhR activation were investigated in vitro and in ileostomy subjects. For the in vitro study, broccoli florets were divided into 5 treatment groups: raw, steamed ( $t=3 \mathrm{~min}$ and $t=6 \mathrm{~min}$ ) and boiled ( $t=3 \mathrm{~min}$ and $t=6 \mathrm{~min}$ ) and then digested in vitro with different gastric $\mathrm{pH}$. In the in vivo ileostomy study, 8 subjects received a broccoli soup or a broccoli soup plus myrosinase source. AhR activation was measured in both in vitro and in vivo samples by using HepG2-Lucia ${ }^{\text {TM }}$ AhR reporter cells. Cooking broccoli reduced the AhR activation measured after gastric digestion in vitro, but no effect of gastric $\mathrm{pH}$ was found. Indole AhR ligands were not detected or detected at very low levels both after intestinal in vitro digestion and in the ileostomy patient samples, which resulted in no AhR activation. AhR activation by glucosinolate derivatives present in broccoli is modulated by several factors including cooking method, cooking time and digestion. Broccoli consumption resulted in AhR activation after the oral and gastric phase in vitro. In contrast, after the small intestinal phase of both in vitro and in vivo samples, no AhR activation was found. This suggests that the evaluation of the relevance of dietary glucosinolates for AhR modulation in the gut cannot prescind from the way broccoli is processed, and that broccoli consumption does not necessarily produce substantial amounts of AhR ligands in the large intestine.

Keywords: Aryl hydrocarbon Receptor, Broccoli, in vitro digestion, ileostomy, thermal treatment 


\section{Introduction}

Health benefits of consuming broccoli and cabbage, which belong to the genus Brassica, have been partly attributed to phytochemicals such as glucosinolates ${ }^{1}$. Recently, broccoli (Brassica oleracea) consumption was shown to alter the microbiota ${ }^{2}$, and attenuate chemically induced colitis in mice through an Aryl hydrocarbon Receptor (AhR)-dependent pathway ${ }^{3}$. Intestinal sources of AhR play an important role in immune function in the gut by recruiting intraepithelial lymphocytes to the epithelium, enhancing the gut defences, triggering anti-inflammatory pathways and controlling microbial load and composition through innate lymphoid cell production of IL-22 $2-6$.

Brassica vegetables are rich in glucosinolates, which can be converted into AhR ligands by myrosinase in the vegetal tissues ${ }^{4,7}$. Myrosinase is a plant enzyme that can enzymatically hydrolyse the chemically stable glucosinolates ${ }^{8}$. Myrosinase and glucosinolates are physically separated from each other in intact vegetable tissues, but mastication in the oral cavity allows them to come into contact with each other, promoting enzymatic hydrolysis of glucosinolates into a variety of breakdown products (BP)9. Glucosinolate hydrolysis is influenced by many endogenous factors like $\mathrm{pH}$, ascorbic acid concentration, and exogenous factors like storage and processing conditions, which can affect myrosinase activity and breakdown product formation? At low pH, nitriles are the main BP from glucosinolates. Additionally, nitriles can be produced during autolysis of fresh plant material. When ascorbic acid is present at a pH between 4 and 7 , ascorbigen and thiocyanates will be the main BP of indole glucosinolates. At pH 6-7, the main BP are isothiocyanates (ITC), however, although some are stable, ITC products of glucosinolates containing an indole moiety as side chain or a $\beta$-hydroxylated side chain, like glucobrassicin, are unstable. $\beta$-hydroxy-ITCs spontaneously cyclise to oxazolidine-2-thiones, while indole ITCs undergo lysis, resulting in the formation of a corresponding alcohol, like indole-3-carbinol (I3C) ${ }^{8}$. The latter subsequently condenses in di-, tri- and tetramers, such as indolo-[3,2b]carbazole (ICZ), cyclic triindole (CT) and 3,3'-diindolylmethane (DIM) under relatively mild acidic conditions ${ }^{10,11}$. Besides, indole-3-acetonitrile (I3N) can be formed as another BP of glucobrassicin, depending on the amount of epithiospecifier protein (ESP) ${ }^{12}$. The biological functions of $\mathrm{I} 3 \mathrm{C}$ include reducing DNA-adduct formation, inducing apoptosis and inhibiting tumour growth ${ }^{13,14}$. It is also a weak AhR ligand, while the products of the acid-catalyzed oligomerization DIM and ICZ have a high AhR binding capacity ${ }^{15}$.

Recent studies have shown that broccoli consumption improves intestinal immune functioning and can alter the host's microbiota, which might affect the production of BP and related condensation products from glucobrassicin and modulate AhR activation ${ }^{3,5,13}$. Broccoli is commonly cooked before consumption, which influences glucosinolate degradation and conversion into AhR ligands. The formation of AhR ligands is influenced by gastric $\mathrm{pH}^{16}$. The 
gastric $\mathrm{pH}$ can be influenced by fasted or fed state, but also by different food products when combined in a meal, which can act as buffer in the gastric environment ${ }^{16-18}$.

The aim of this research was to investigate the effect of broccoli processing and consumption on in vitro and in vivo AhR activation. We investigated the effect of temperature, cooking method (boiling versus steaming) and gastric $\mathrm{pH}$ on AhR activation by glucosinolate derivatives from broccoli in vitro. To investigate AhR activation in humans and the effect of absorption, which is lacking in in vitro systems, we also measured AhR activity in the ileal fluid samples collected from ileostomy subjects after consumption of a broccoli soup with or without supplementation of an exogenous source of active myrosinase. 


\section{Materials and Methods}

\subsection{Sample preparation and thermal treatments}

\subsubsection{Broccoli preparation and thermal treatments for in vitro study}

One batch of broccoli was purchased at the local supermarket (The Netherlands). The stems of the broccoli were removed and the florets were cut into pieces of approximately $3 \times 3 \times 3 \mathrm{~cm}$. The florets were equally divided into 5 groups and treated as follows: raw (group 1), boiled for 3 min (group 2), boiled for 6 min (group 3), steamed for 3 min (group 4) and steamed for 6 min (group 5). Broccoli (265 g) was boiled in $1.4 \mathrm{~L}$ water (groups 2 and 3), or $240 \mathrm{~g}$ broccoli was steamed in a steam oven (groups 4 and 5), for 3 or 6 min. Thereafter, the broccoli was drained of water for 5 min, immediately frozen by liquid nitrogen and freeze-dried. The trays used for freeze-drying were weighed before and after freeze-drying to determine the loss in water content of the samples. After freeze-drying, the samples were milled ( 2 min cooling time, 7 min milling time) into a fine powder, using a freeze miller (SpexEuropa, SPEX SamplePrep) and stored at $-20^{\circ} \mathrm{C}$ until further analysis.

\subsubsection{Broccoli preparation and thermal treatments for in vivo study}

Beneforte super broccoli was supplied by Staples Vegetables Ltd (Boston, Lincolnshire, UK) and mustard seeds were sourced from a local supermarket (UK). Broccoli florets were cut (around 4 to $5 \mathrm{~cm}$ from top) and combined thoroughly. Fifty gram portions were placed and vacuum-packed in flexible polyethylene bags (LDPE) with dimensions of $24 \times 24 \mathrm{~cm}$ to stop glucoraphanin leaching into the processing water. Using a thermostatic water bath, broccoli was cooked at $100^{\circ} \mathrm{C}$ for $12 \mathrm{~min}$. Mustard seeds were ground and added as a powder as source of myrosinase (at $2 \% \mathrm{w} / \mathrm{w}$ ) at the end of the soup preparation process $\left(\sim 60^{\circ} \mathrm{C}\right.$ ). All samples of raw and cooked super broccoli were frozen in dry ice before storage at $-80^{\circ} \mathrm{C}$. Following this, samples were lyophilized (VirTis SP Scientific, UK), ground using a coffee grinder and sieved (30 mesh). Samples were stored at $-20^{\circ} \mathrm{C}$ until soup preparation.

The preparation of the soups included the following steps (Table 1): broccoli powder was added to boiling water $(400 \mathrm{~mL}$ ) and cooked for $2 \mathrm{~min}$. The rest of the ingredients, except mustard seed powder, were dissolved in $300 \mathrm{~mL}$ cold water and then added to the boiling soup. The soup was boiled for 3 additional min to inactivate ESP ${ }^{12}$. Afterwards, the soup was left to cool down to $60^{\circ} \mathrm{C}$, followed by addition of the mustard seed powder. The soup was left in the water bath at $60^{\circ} \mathrm{C}$ for no more than two hours until serving of $200 \mathrm{~mL}$ per subject. 
Table 1. Ingredients of $200 \mathrm{~mL}$ broccoli soup.

\begin{tabular}{lc}
\hline Broccoli soup (200 $\mathbf{~ L L})$ & Gram \\
\hline Broccoli powder & 11 \\
Cheese powder (parmesan cheese) & 5 \\
Sauce mix ${ }^{1}$ & 11 \\
Salt & 0.3 \\
Mustard seed powder & 0.16 \\
\hline WWheatflour, cheddar cheese powder (16\%), cheese powder (16\%), maize \\
starch, skimmed milk powder, salt, yeast extract, flavouring, maltodextrin, \\
whey powder, mustard powder, colours (curcumin, paprika extract).
\end{tabular}

\subsection{Determination of glucosinolate content and myrosinase activity}

Glucosinolates of the thermally treated broccoli samples were extracted according to Oliviero et al. ${ }^{19}$ with and without the pre-addition of water to estimate the myrosinase activity. To determine the glucosinolates concentrations, $0.1 \mathrm{~g}$ freeze dried broccoli was mixed with $2.4 \mathrm{~mL}$ $100 \%$ methanol $\left(75^{\circ} \mathrm{C}\right)$ and $200 \mu \mathrm{L} 3 \mathrm{mM}$ glucotropaeolin (Sigma-Aldrich), after which the samples were incubated in a water bath at $75^{\circ} \mathrm{C}$ for $5 \mathrm{~min}$. The samples were centrifuged at $1363 \mathrm{~g}$ for $10 \mathrm{~min}$ and the supernatants were collected, while the pellets were re-extracted twice by adding $2 \mathrm{~mL} 70 \%$ methanol $\left(75^{\circ} \mathrm{C}\right)$. All supernatants were combined and stored at $-20{ }^{\circ} \mathrm{C}$ until desulphation. Glucosinolates were converted into desulphoglucosinolates according to Oerlemans et al. ${ }^{20}$. The extracted desulphated glucosinolates were separated using an Ultimate 3000 U-HPLC (Thermo Scientific, Bremen, Germany) with Licospher 100 RP 18 column (125x $4 \mathrm{~mm}$ ) with an injection volume of $20 \mu \mathrm{L}$ and a flow rate of $1 \mathrm{~mL} / \mathrm{min}$. Two eluents were used, eluent A (100 \% milliQ) and eluent B (100\% Acetonitrile). The HPLC ran for 25 min with a gradient elution of $100 \%$ A for 2 min, $92 \%$ A for 5.5 min, $75 \%$ A for 10.5 min and back to 100 $\%$ A for 2 min. The peaks were measured with a UV detector at a wavelength of $229 \mathrm{~nm}$. The glucosinolates were identified by means of their absorption spectra and quantified by comparing their area to the area of a standard glucosinolate; glucotropaeolin (benzylglucosinolate).

In order to estimate myrosinase activity, the thermally treated broccoli samples were pre-incubated with water for 5 min, after which the glucobrassicin concentration was measured as described above. The myrosinase activity was expressed as percent of glucobrassicin reduction where the glucobrassicin concentration with and without water addition was compared.

\subsection{Digestion: in vitro and in vivo}

\subsubsection{INFOGEST in vitro digestion}

To simulate complete human digestion, the protocol of Minekus et al., (2014) was used with some modifications ${ }^{21}$. In short, $0.5 \mathrm{~g}$ freeze dried broccoli (samples from group 1 to 5) was added to $4.5 \mathrm{~g}$ milliQ and incubated for $5 \mathrm{~min}$ at $37^{\circ} \mathrm{C}$ while rotating to simulate the oral phase. This was 
mixed with $3.5 \mathrm{~mL}$ simulated salivary fluid (1.475 mL milliQ, $25 \mu \mathrm{L} 0.3 \mathrm{M} \mathrm{CaCl}_{2}$ ) and incubated for 2 min at $37^{\circ} \mathrm{C}$ while rotating at speed 10 (Salm en Kipp B.V.). Subsequently, the oral bolus went to the gastric phase where the $\mathrm{pH}$ was decreased to $\mathrm{pH} 3$ with $\mathrm{HCl}$ and mixed with $7.5 \mathrm{~mL}$ simulated gastric fluid ( $5 \mu \mathrm{L} 0.3 \mathrm{M} \mathrm{CaCl}_{2}$, milliQ and $1.6 \mathrm{~mL} 2000 \mathrm{U} / \mathrm{mL}$ pepsin, Sigma-Aldrich), this mixture was incubated for 2 hours at $37^{\circ} \mathrm{C}$ while rotating. Subsequently, the food bolus went to the intestinal phase where the $\mathrm{pH}$ was increased to $\mathrm{pH} 7$ using $\mathrm{NaOH}$ and mixed with $11 \mathrm{~mL}$ simulated intestinal fluid $\left(40 \mu \mathrm{L} 0.3 \mathrm{M} \mathrm{CaCl}_{2}\right.$, milliQ and pancreatin; based on trypsin activity of $100 \mathrm{U} / \mathrm{mL}$ in final mixture (Sigma-Aldrich)). Again, this was incubated for 2 hours at $37^{\circ} \mathrm{C}$ while rotating. Samples were taken after the gastric and intestinal phases and centrifuged at $9000 \mathrm{~g}$ for $12 \mathrm{~min}$. The supernatants were stored at $-20^{\circ} \mathrm{C}$ until further analysis.

\subsubsection{In vitro gastric digestion of broccoli samples}

To simulate human gastric digestion and vary the gastric $\mathrm{pH}$, a simplified in vitro digestion method was used. Freeze-dried broccoli powder (0.5 g samples from groups 1-5) was added to $4.5 \mathrm{~mL}$ milliQ and incubated for $5 \mathrm{~min}$ at $37{ }^{\circ} \mathrm{C}$ while rotating. Subsequently, $5 \mathrm{~mL}$ dichloromethane (Actu-All Chemicals) was added and incubated for 2 min at room temperature while shaking, followed by centrifugation at $2670 \mathrm{~g}$ for $5 \mathrm{~min}$. This procedure was repeated twice. Supernatant was collected and dichloromethane was evaporated using $\mathrm{N}_{2}$, after which $1 \mathrm{~mL}$ acetonitrile (Actu-All Chemicals) was added to re-dissolve the active compounds. This was mixed with 6.5 $\mathrm{mL}$ milliQ, and the $\mathrm{pH}$ was decreased to either $\mathrm{pH}$ 2, 3 or 5 using $\mathrm{HCl}$. Subsequently, milliQ was added to reach a final volume of $10 \mathrm{~mL}$ with the correct $\mathrm{pH}$ and the samples were incubated for 2 hours at $37^{\circ} \mathrm{C}$ while rotating. After incubation, the $\mathrm{pH}$ was increased to $\mathrm{pH} 7$ using $\mathrm{NaOH}$ and the samples were centrifuged for $12 \mathrm{~min}$ at $4816 \mathrm{~g}$. The supernatants were stored at $-20^{\circ} \mathrm{C}$ until further analysis.

\subsubsection{In vivo dietary intervention study with ileostomy patients}

To compare the in vitro broccoli digestion with in vivo digestion, ileostomy patients were used. lleostomy studies provide insight into the processes of biotransformation and absorption in the upper gastrointestinal tract that impact the phytochemicals in the ingested plant material, in the case of broccoli potentially influencing AhR bioactivity and health benefits ${ }^{22}$. Ethical approval (14/SC/1326) for the ileostomy feeding study was received from South Central - Hampshire Research Ethics Committee and Ulster University. All participants gave written informed consent and the study was conducted in accordance with the Helsinki Declaration. Participants were recruited between January 2015 and February 2015 from clinics at Altnagelvin Hospital. The intervention study ran between March 2015 and April 2015. The study was registered at clinicaltrials.gov (Identifier: NCT04113928). The study was conducted in 11 ileostomy subjects (7 males, 4 females) age range 32-63 years, who had undergone terminal ileostomies, were at least 1.5 years post-operative prior to the study and were non-smokers. 
The study was an acute randomised, single-blind, placebo-controlled crossover feeding trial with a 1-week washout period. After obtaining informed consent, participants were randomly assigned, in blocks of four using a random-number generator (www.randomization.com), to either the intervention or the control. In total, 12 participants were randomised to 2 groups. One participant subsequently withdrew from the study prior to sampling. Participants were asked to follow a restriction diet, avoiding dark green leafy vegetable and mustard-like vegetables especially broccoli, cabbage, Brussel sprouts, watercress, rocket, spinach, onions, spring onions, radish, horseradish for $48 \mathrm{~h}$ before each clinic visit. Following an overnight fast, the participants provided an ileal fluid sample ( $\mathrm{T}$ h) then consumed $200 \mathrm{~mL}$ of freshly prepared broccoli soup (described above) with or without addition of mustard seed (containing myrosinase). A second ileal fluid sample was collected 4 hours post consumption ( $T 4 \mathrm{~h}$ ). The ileal fluid samples were collected and processed as described in McDougall et al. ${ }^{23}$. Weights and $\mathrm{pH}$ of the ileal fluid were recorded, before dilution with ice cold distilled water as required dependent on viscosity, before being homogenised in a chilled Waring blender for 30 seconds, and storage of aliquots at $-80^{\circ} \mathrm{C}$ in preparation for subsequent analysis. A random subset of 8 participants were used to assess AhR activation, where 4 subjects started with the broccoli soup and the other 4 with the broccoli soup + mustard seeds.

Freeze-dried ileostomy sample $(0.5 \mathrm{~g}$ ) was extracted using dichloromethane as described in section 2.3.2. After evaporation, the compounds were redissolved in $2 \mathrm{~mL} M E M$ growth medium (Gibco, USA) and centrifuged at $3000 \mathrm{~g}$ for $5 \mathrm{~min}$. The supernatant was added in a $1 \%$ final concentration to HepG2-Lucia ${ }^{\text {TM }}$ AhR reporter cells (InvivoGen, France) to measure AhR activation (section 2.5). In addition, cytotoxicity was measured using the CytoTox 96 NonRadioactive Cytotoxicity Assay according to the manufacturers instructions (Promega, USA).

Samples were further processed using solid phase extraction (SPE), using Strata-E C18 reversed phase columns (Phenomenex), in order to clean up for quantification. The columns were conditioned with $1 \mathrm{~mL}$ methanol and equilibrated with $1 \mathrm{~mL}$ milliQ. Pre-extracted ileostomy sample $(100 \mu \mathrm{L})$ was loaded, after which $1 \mathrm{~mL} 5 \%$ methanol in milliQ was loaded. The column was dried under vacuum for $5 \mathrm{~min}$ and the sample was eluted with $1 \mathrm{~mL}$ methanol. Afterwards, the methanol was evaporated and samples were redissolved in $150 \mu \mathrm{L}$ milliQ until quantification using LC-MS/MS (2.6).

\subsection{AhR activation}

HepG2-Lucia'M AhR reporter cells (InvivoGen) were grown according to the manufactures instructions in Minimum Essential Medium Eagle - Alpha Modification (a-MEM; Gibco USA), 10\% (v/v) heat inactivated fetal calf serum (Gibco), 1x non-essential amino acids (Gibco),

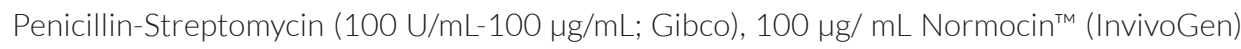
and $100 \mu \mathrm{g} / \mathrm{mL}$ Zeocin $^{\text {TM }}$ (InvivoGen). To measure AhR activation, $20 \mu \mathrm{l} \mathrm{sample} \mathrm{was} \mathrm{added}$ 
in triplicate to a 96-wells plate (Corning, USA). Thereafter, $180 \mu \mathrm{l}$ cell suspension ( 20,000 cells/well) was added to each well and the plate was incubated for 48 hours at $37^{\circ} \mathrm{C}$ in a $\mathrm{CO}_{2}$ incubator. After incubation, $20 \mu \mathrm{L}$ stimulated cell supernatant was transferred into a white walled clear bottom 96-wells plate (Corning), followed by addition of $50 \mu \mathrm{L}$ QUANTI-Luc ${ }^{\text {TM }}$ (InvivoGen). Luminescence was measured immediately using a Spectramax M5 (Molecular devices, USA). AhR activation was expressed as percentage of the activity of the positive control ( $5 \mu \mathrm{M} \beta$-naphthoflavone in DMSO). Results of AhR activation were corrected for their corresponding negative controls, either DMSO or medium.

\subsection{Detection of I3C and DIM}

To detect I3C and DIM in the ileostomy samples, the samples were extracted by the procedure described previously by Domingues-Perles et al., with some modifications ${ }^{24}$. Briefly, the frozen ileal fluid samples were thawed and vortexed, and duplicate $5.0 \pm 0.1 \mathrm{~g}$ samples were weighed into $15 \mathrm{~mL}$ centrifuge tubes. These samples were extracted using $2 \mathrm{~mL}$ methanol: water (80:20, $\mathrm{v} / \mathrm{v}$ ) with $1 \%$ formic acid. The tubes were vortexed for $3 \times 30 \mathrm{~s}$ and then sonicated in a water bath for $1 \mathrm{~min}$. All procedures were carried out at $5^{\circ} \mathrm{C}$. After centrifugation ( $4000 \mathrm{~g}, 15 \mathrm{~min}$, $4^{\circ} \mathrm{C}$ ), the supernatants were transferred into new tubes. The pellets were extracted twice using $3 \mathrm{~mL}$ of the same solution and the supernatants combined and vortexed. The extracts were further extracted using SPE Strata-X cartridges (33u Polymeric Strong Cation), following the manufacturer's instructions. Cartridges were conditioned with $2 \mathrm{~mL}$ of $\mathrm{MeOH}$ and equilibrated with $2 \mathrm{~mL}$ ultrapure water/formic acid (98:2, v/v). After this step, the extracted ileal simples were applied to the column. Then, the SPE cartridges were washed with water/formic acid (98:2, v/v) and aspirated until dryness. The target analytes including I3C and DIM, were eluted with $2 \mathrm{~mL}$ of $\mathrm{MeOH} /$ formic acid (98:2, v/v) and dried using a Speed Vac concentrator. The extract was reconstituted with $200 \mu \mathrm{L}$ of solvent A/B (90:10, v/v) until analysis.

The targeted analysis of I3C and DIM was performed on an Ultra High Performance Liquid Chromatography Photo Diode Array mass spectrometer system (UHPLC-PDA-MS; Thermo Scientific, USA) comprising of a UHPLC pump, a PDA detector scanning from 200 to $600 \mathrm{~nm}$, and an autosampler operating at $4{ }^{\circ} \mathrm{C}$ (Dionex Ultimate 3000 RS, Thermo Corporation). Separations of I3C and DIM were based on a $100 \times 2.1$ mm i.d. 1.8 um Zorbax SB-C18 RRHD column (Agilent, Santa Clara, CA) preceded with a precolumn with the same stationary phase (Waters, Spain) which was maintained at $40^{\circ} \mathrm{C}$ and eluted using two mobile phases: A: deionized water with 13 $\mathrm{mM}$ of ammonium acetate $(\mathrm{pH} 4.0$ with acetic acid) and B: acetonitrile with $1 \%$ acetic acid, over the course of 20 min at $0.2 \mathrm{~mL} / \mathrm{min}$. The gradient started with $3 \%$ of B rising $20 \%$ B in 5 min, then rising $52 \%$ B in 3 min following $95 \%$ of B after 4.5 min, maintained for 2.5 min and finally return to $3 \% \mathrm{~B}$ in $1 \mathrm{~min}$ and maintained during 4 min to equilibrate the column to the initial conditions. After passing through the flow cell of the PDA detector, the eluate went directly to an Exactive Orbitrap mass spectrometer (Thermo Scientific) fitted with a Heated Electrospray 
Ionization Probe (HESI) operating in positive ionization mode. Full scans were recorder in $\mathrm{m} / \mathrm{z}$ range from 100 to 800 with a resolution of $50.000 \mathrm{~Hz}$ and with a full AGC target of 100000 charges, using 2 microscans. Analyses were also based on scans with in-source collision-induced dissociation (CID) at $25.0 \mathrm{eV}$. MS experiment condition with HESI in positive ionization mode was: capillary temperature $325^{\circ} \mathrm{C}$, heater temperature $300^{\circ} \mathrm{C}$, sheath gas 18 units, auxiliary gas 2 units, and the spray voltage $4.0 \mathrm{kv}$. Targeted identifications of I3C and DIM were achieved by comparing the exact mass and the retention time with available standards. Quantification of the compounds was carried out by selecting the theoretical exact mass of the molecular ion by reference to standard curves prepared in diluted ileal fluid extract.

\subsection{Detection of indole derivatives}

To detect indole derivatives in the ileostomy samples, a Thermo UltiMate 3000 U-HPLC system coupled to a TSQ Quantum triple quadrupole mass spectrometer (Thermo Fisher Scientific, Germany) was used. Chromatography was carried out with a Phenomenex Kinetex $1.7 \mu \mathrm{m}$ EVO C18 100 A LC column $(100 \times 2.1 \mathrm{~mm})$ maintained at $45^{\circ} \mathrm{C}$. Mobile phases consisted of milliQ (A) and methanol (B) at a flow rate of $0.3 \mathrm{~mL} / \mathrm{min}$ with the following gradient: $0-2 \mathrm{~min}, 0.1 \% \mathrm{~B}$; 2-6 min, 0.1-25 \% B; 6-10 min, 25-80 \% B; 10-12 min, 80-90 \% B; 12-15 min, 90 \% B; 15-16 min, $90-0.1 \%$ B; then re-equilibration for 10 min. The samples were kept at $5{ }^{\circ} \mathrm{C}$ in an auto sampler (WPS-3000 TRS, Thermo Fisher Scientific) throughout the run and $10 \mu \mathrm{L}$ per sample was injected for analysis under positive mode. The electrospray sources parameters were set as follows: spray voltage, $4.5 \mathrm{kV}$; capillary temperature, $275^{\circ} \mathrm{C}$; sheath gas, 40 arbitrary units; auxiliary gas, $\mathrm{O}$ arbitrary units. Table $\mathrm{S} 1$ (supplementary material) listed the exact mass precursor ions, exact mass product ions, retention time (RT), collision energy (CE), and tube lens ( $T L$ ) for each metabolite. Data analysis was performed using Thermo Xcalibur 4.0 software (Thermo Fisher Scientific).

\subsection{Statistical analysis}

All statistical analyses were performed using Graphpad Prism 5 (La Jolla, USA). Results were expressed as mean \pm standard error of the mean (SEM). Differences were considered significant when $p<0.05$. Differences in glucosinolates were tested using a one-way ANOVA followed by a Tukey post-hoc test. AhR activation among the differently treated broccoli, were tested using a one-way ANOVA followed by a Tukey post-hoc analysis. AhR activation among different $\mathrm{pH}$ was tested using a repeated measures ANOVA followed by a Bonferroni post-hoc test. The samples before and after in vitro digestion were tested using a one-way repeated measures ANOVA followed by a Bonferroni post-hoc test. Letters above bars represent classes of statistically significant different responses compared to each sample. Each graph bar with the same letter is not statistically significant different. 


\section{Results and Discussion}

\subsection{Glucobrassicin concentration and myrosinase activity}

We quantified the level of glucobrassicin in the raw and thermally treated broccoli samples (Figure 1a). Glucobrassicin is the only glucosinolate responsible for AhR activation as it can be converted into AhR ligands DIM and ICZ via I3C 3,15,25,26. The glucobrassicin concentration in the 3 min steamed broccoli was the highest, with $466 \mu \mathrm{mol} / 100 \mathrm{~g}$, which was significantly higher than the raw brocolli (280 $\mu \mathrm{mol} / 100 \mathrm{~g}, \mathrm{p}<0.05)$ and boiled broccoli samples (293 and 199 $\mu \mathrm{mol} / 100 \mathrm{~g}$ after 3 and 6 min boiling, $p<0.05$ and $p<0.01$ respectively). The concentration of glucobrassicin is highly dependent on the type and batch of broccoli, and our values lie within the previously reported ranges such as those reported by Oliviero et al., and Vallejo et al.19,27. The loss of total glucosinolates during heating has been previously reported and attributed to leaching of glucosinolates into the cooking water (up to $90 \%$ ) $^{27-32}$, and a time-dependent glucosinolate loss by thermal treatment ${ }^{19,33,34}$. A lower amount of glucobrassicin was measured in raw broccoli compared to steamed broccoli (3 min, $p<0.05$ ), due to an increased extractability in the thermally treated broccoli matrix, as reported previously ${ }^{19,35,36}$.

a

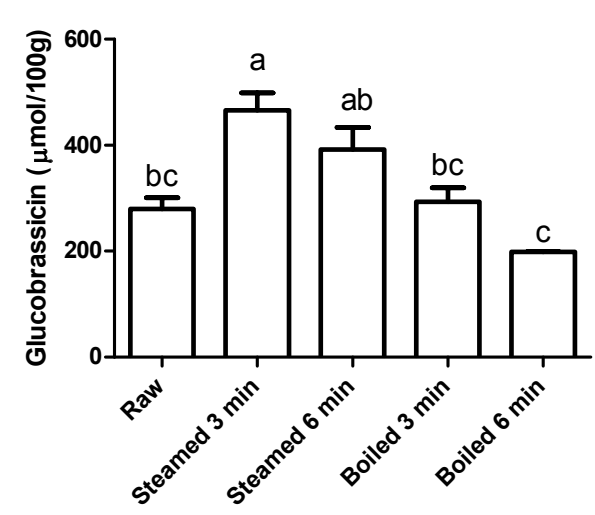

b

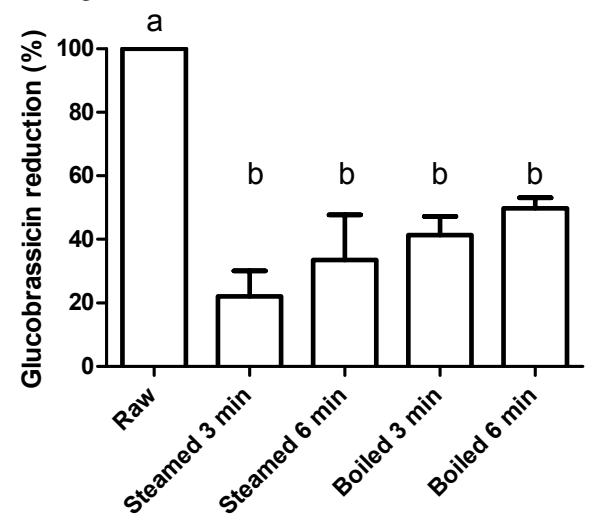

Figure 1. Effect of cooking method. (a) Glucobrassicin concentration ( $\mu \mathrm{mol} / \mathrm{l} 100 \mathrm{~g}$ freeze dried broccoli) for raw, steamed (3 or 6 min) and boiled (3 or 6 min) broccoli $(n=2)$. (b) Glucobrassicin reduction (\%) with addition of water before extraction compared to no addition of water before extraction $(n=3)$. A one-way ANOVA followed by a Tukey post-hoc test was used. Letters above bars represent classes of statistically significant different responses compared to each sample. Each graph bar with the same letter is not statistically significant different. 
To estimate the myrosinase activity in the thermally treated samples, extraction was performed with pre-incubation of water, in order to facilitate hydrolysis of glucosinolates by myrosinase. Figure $1 \mathrm{~b}$ shows the glucobrassicin reduction in the thermally treated samples after the addition of water. The raw broccoli had the highest myrosinase activity, as there was no glucobrassicin found after incubation with water $(p<0.05)$. All thermal treatments reduced the myrosinase activity, with no significant differences between the treatments. Myrosinase was not completely inactivated, but a reduction of $\geq 50 \%$ was observed. From this we can conclude that the thermal treatments retained a significant myrosinase activity and that there were no differences observed among the thermal treatments.

\subsection{AhR activation by undigested broccoli}

Before testing the AhR activation of DIM and I3C in the differently treated broccoli samples, we checked for AhR activation of I3C and DIM in a range of concentrations using a newly developed AhR reporter cell line (Figure 2a and 2b). DIM gave higher AhR activation at a lower concentration compared to I3C, namely $75 \%$ of the $\beta$-naphthoflavone positive control, in the concentration range 25 to $500 \mu \mathrm{M}$, while I3C only reached this level of activation when cells were stimulated with concentrations ranging from 100 to $250 \mu \mathrm{M}$. This implies that DIM is a more potent AhR ligand, which is confirmed by literature ${ }^{3,11}$, but also I3C can activate AhR in a dose-dependent manner. Beside DIM and I3C, also indole-3-acetonitrile (I3N) was previously shown to be a weak AhR ligand, together with ICZ, the latter being the most potent AhR ligand among those obtained from glucobrassicin breakdown ${ }^{37,38}$.
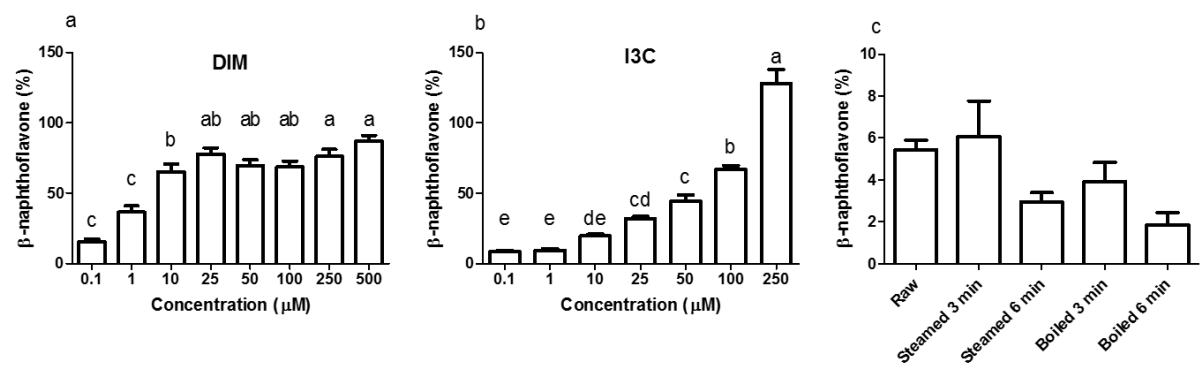

Figure 2. AhR activation measured using HepG2-LuciaTM AhR cells, with luciferase production as read out, expressed as percentage of positive control ( $\beta$-naphthoflavone, $5 \mu \mathrm{M}$ ). a) 3,3'-Di-indolylmethane (DIM), b) Indole-3-carbinol (I3C). c) undigested raw, 3 and 6 min steamed, and 3 and 6 min boiled broccoli (1 $\mathrm{mg} / \mathrm{mL}$ ). Data is expressed as mean $\pm \mathrm{SEM}, n=3$. A one-way ANOVA followed by a Tukey post-hoc test was used. Letters above bars represent classes of statistically significant different responses compared to each sample. Each graph bar with the same letter is not statistically different. 
When undigested broccoli samples were tested, activation of AhR was relatively low but the highest AhR activation was observed in the raw broccoli and broccoli steamed for 3 min. The thermal treatments reduced the AhR activation considerably $(p<0.1)$, with a general trend of AhR activation decreasing as the intensity of the thermal treatment increases (Figure 2c). However, in line with the results above, steaming for 3 min and raw broccoli showed the same level of AhR activation. Boiling is known to result in significant losses of glucosinolates in the cooking water, as can be seen in Figure 1 by comparing the glucobrassicin content of broccoli steamed and boiled for a comparable time. The loss of glucosinolates by cooking meant that less glucosinolates were available to be hydrolysed into AhR ligands. Loss of myrosinase activity can decrease glucosinolate hydrolysis during mastication and digestion, which decreases AhR activation 28,39 . Besides the amount of glucosinolates present and the residual myrosinase activity, AhR activation in broccoli can be modulated by the residual epithiospecifier protein (ESP) activity ${ }^{12}$.

ESP is a myrosinase co-factor, that can favour the formation of nitriles over isothiocyanates in an iron-dependent manner ${ }^{12}$. In addition, presence of the epithiospecifier modifier protein (ESM1), can inhibit the ESP mediated nitrile formation leading to an increase in ITC ${ }^{40}$. Although I3C and I3N both are AhR ligands ${ }^{38}$, only I3C can be further metabolised into the more potent AhR ligands DIM and ICZ ${ }^{41}$. Compared with raw broccoli, a mild steaming can inactivate ESP leaving enough myrosinase activity to produce I3C, whereas in raw broccoli the largely intact ESP activity may direct glucobrassicin mostly towards I3N ${ }^{12}$. The impact of ESP on the AhR activation from broccoli products is difficult to predict, mostly because the relative potency of I3C and I3N as AhR ligands has never been compared. Our results show that light steaming did not decrease AhR activation and that a partial inactivation of myrosinase does not necessarily compromise formation of AhR ligands from glucobrassicin in broccoli. Therefore, we hypothesise that the lower AhR activation observed after more intense thermal treatments is likely the result of loss of glucosinolates rather than of myrosinase activity (Figure 2c).

\subsection{The effect of gastric $\mathrm{pH}$ on AhR activation}

In a separate set of experiments, we sought to investigate the effect of gastric $\mathrm{pH}$ on the formation of AhR ligands and therefore potential AhR ligand activation by broccoli. Therefore, all samples were digested by a simplified in vitro gastric digestion with various $\mathrm{pHs}(\mathrm{pH} 2,3$ and 5). As reported in Figure 3, raw and 3 min steamed broccoli show significantly higher AhR activation at pH 2 and 3 compared to 3 and 6 min boiled and 6 min steamed broccoli $(p<0.01)$. At $\mathrm{pH}$ 5, the only significant difference was between raw and boiled broccoli, while steamed broccoli did not show a higher activation compared to boiled broccoli. These results are in line with the AhR activation of the undigested samples (Figure 2), where it was observed that raw and 3 min steaming results in the highest AhR activation. 
The different $\mathrm{pH}$ tested did not significantly influence AhR activation suggesting that in vivo the gastric $\mathrm{pH}$ will not affect the formation of AhR ligands in the stomach. Previous research showed that ITC formation is favoured at neutral $\mathrm{pH}$ ( $\mathrm{pH} 4-7)$, while nitrile formation is favoured at acidic $\mathrm{pH}^{8,10,16,42,43}$. DIM and ICZ are more potent AhR ligands than I3C and I3N $\mathrm{N}^{38}$. As DIM and ICZ are formed from I3C, one could conclude that AhR activation should be highest at neutral $\mathrm{pH}^{8,15,41}$. However, De Kruif et al., (1991) found that the highest amount of DIM formed was found at pH 4-517, whereas Grose \& Bjeldanes, (1992) found that the highest amount of ICZ formed was found at $\mathrm{pH}$ close to neutrality ${ }^{44}$. In our study, we might have favoured the conversion of isothiocyanates by including an oral phase at neutral pH. Overall, it is difficult to correlate the expected effect of $\mathrm{pH}$ on glucobrassicin breakdown products to AhR activation because the observed level of activation is a complex balance of relative levels of I3C, I3N, DIM and ICZ.

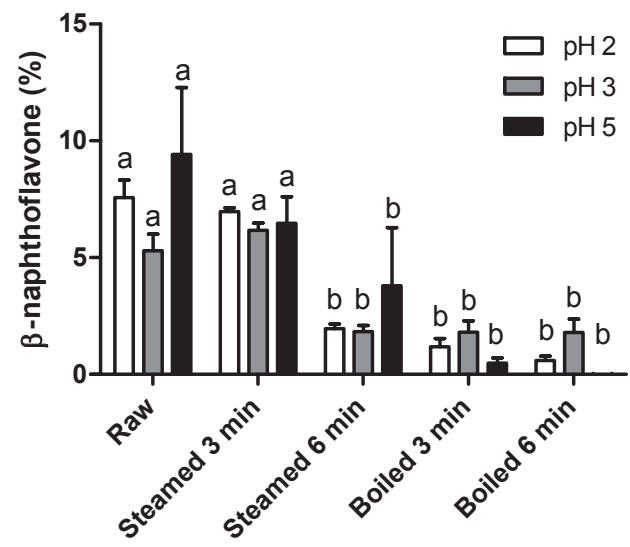

Figure 3. Effect of gastric pH. AhR activation measured using HepG2-Lucia ${ }^{\text {TM }}$ AhR cells, with luciferase production as read out, expressed as percentage of positive control ( $\beta$-naphthoflavone, $5 \mu \mathrm{M}$ ) in raw broccoli, steamed broccoli ( $t=3 \mathrm{~min}, t=6 \mathrm{~min}$ ) and boiled broccoli $(t=3 \mathrm{~min}, t=6 \mathrm{~min})$, digested at different $\mathrm{pH}$. Data is expressed as mean \pm SEM, $n=4$ biological replicates and $n=3$ biological replicates for 6 min steamed broccoli at pH 5. A one-way repeated measures ANOVA followed by a Bonferroni post-hoc test was used. Letters above bars represent classes of statistically significant different responses compared to each sample. Each graph bar with the same letter is not statistically different.

Brassica vegetables are normally consumed as part of a meal. The effect of digestion on glucosinolates and AhR activation may therefore also be influenced by the composition of the meal. Although in our study $\mathrm{pH}$ showed no significant effect, the presence of ferrous ions at acidic $\mathrm{pH}$ increases nitrile formation and could therefore indirectly decrease the formation of DIM and ICZ. Additionally, ascorbic acid is a co-factor of myrosinase and directs hydrolysis towards thiocyanates and ascorbigen formation, which decreases I3C formation and possibly 
decreases AhR activation 8,16,42,45. As mentioned previously, formation of AhR ligands is theoretically higher at neutral $\mathrm{pH}$, although the literature is not completely in agreement. The $\mathrm{pH}$ in an empty stomach can be less than 2, but when food enters the stomach, the buffering effect exerted by food components, primarily by proteins, can increase the $\mathrm{pH}$ to 5 or even higher ${ }^{17,46}$. This would imply that it could be beneficial to consume raw broccoli in combination with a protein rich product, like meat, although in our study no clear effects of gastric $\mathrm{pH}$ were observed, contradicting previous studies.

\subsection{In vitro digestion: gastric and small intestinal effects on AhR}

To study the effect of the intestinal digestion on AhR activation, all broccoli samples were digested using the INFOGEST standard in vitro digestion protocol with a $\mathrm{pH}$ of 3 during the gastric phase. In Figure 4, we report the AhR activation of the digesta supernatant, i.e. the activation resulting from AhR ligands formed in the broccoli matrix and released from the matrix during the gastric phase. This would represent the AhR ligands available for absorption or activation in the small intestine. AhR activation of raw broccoli during standard digestion was significantly higher after the gastric phase $(p<0.001)$ than the cooked broccoli. Also, 3 min steamed broccoli had a higher AhR activation than both 3 and 6 min boiled $(p<0.05)$. After the intestinal phase, no AhR activation was observed in any of the samples (data not shown). Results in Figure 4 confirm the effect of thermal treatment on formation of AhR ligands from broccoli. However, when results in Figure 4 and Figure 2c are compared (AhR activation by broccoli before digestion), it appears that the gastric step has activated raw broccoli more than the thermally treated broccoli and this can only be related to different initial levels of I3C after the treatment. The results in Figure 2c only show the AhR activation by broccoli in a concentration of $1 \mathrm{mg} / \mathrm{mL}$, whereas the digested samples were diluted with digestive fluids. Therefore, the AhR activation after the gastric phase might be underestimated. Besides, part of the ligands may have remained entrapped in the broccoli matrix and are possibly released upon fermentation of the polysaccharide matrix in the gut.

To explain the absence of AhR activation after the intestinal phase, despite the absence of an absorption step in our static model of digestion, we tested the degradation or further conversion of I3C and DIM at the neutral pH of the small intestine (Figure 5). Results show that there is a degradation of both $13 \mathrm{C}$ and DIM during 2 hours incubation at $\mathrm{pH} 7$ at $37^{\circ} \mathrm{C}$, ranging from $20 \%$ at a low concentration to $5 \%$ at a higher concentration.

We also tested the possible interference of digesta fluids on the cells by testing the AhR activation of I3C spiked in pancreatin. Results showed that AhR activation was completely absent when $40 \mu \mathrm{M}$ I3C was mixed with pancreatin, probably due to cell growth inhibition, which could partially explain the lack of AhR activation found in samples from the in vitro intestinal phase. 
Of course, the dilution of gastric digesta with simulated intestinal fluids during in vitro digestion also needs to be considered but does not account for the complete loss of AhR activation that was observed. On the other hand, AhR ligands may be slowly released from the broccoli matrix during the 2 hours of the intestinal phase, resulting in a lack of AhR activation.

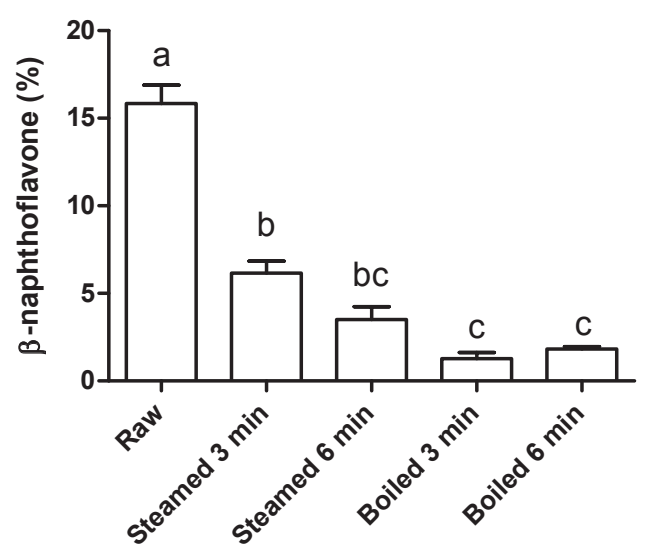

Figure 4. Effect of gastric digestion. AhR activation measured with HepG2-Lucia ${ }^{\text {TM }}$ AhR cells, using luciferase production as read out, expressed as percentage of positive control ( $\beta$-naphthoflavone, 5 $\mu \mathrm{M}$ ). The bioaccessible fraction (supernatant) of glucobrassicin-derived AhR ligands from raw broccoli, steamed broccoli ( $t=3 \mathrm{~min}, t=6 \mathrm{~min}$ ) and boiled broccoli $(t=3 \mathrm{~min}, t=6 \mathrm{~min})$ after the gastric phase using in vitro digestion were tested. Data is expressed as mean \pm SEM with $n=3$ for the raw and steamed and $n=2$ for the boiled broccoli. A one-way ANOVA followed by a Tukey post-hoc test was used. Letters above bars represent classes of statistically significant different responses compared to each sample. Each graph bar with the same letter is not statistically different.

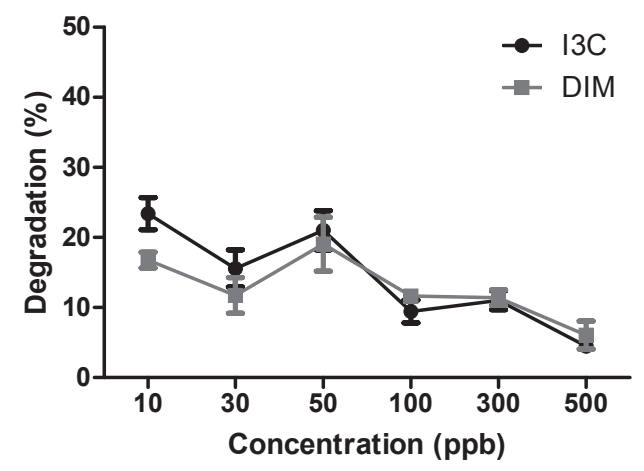

Figure 5. Degradation (\%) of different concentrations of $I 3 \mathrm{C}$ and $\mathrm{DIM}$ at $\mathrm{pH} 7$ at $37^{\circ} \mathrm{C}$ for 2 hours to simulate the in vitro small intestine. Results expressed as mean $\pm \mathrm{SEM}, n=3$. 


\subsection{In vivo digestion: ileal fluids and its effects on AhR activation}

In order to confirm our in vitro data, ileal fluid samples from ileostomy patients were tested for AhR activation. This was the first time that ileal fluid has been tested for their potential to activate AhR. Therefore, these samples may provide useful information on the presence and transformation of AhR ligands during small intestinal digestion, and thus on the potential activation of AhR in the colon. Results show that none of the ileal samples from the 8 ileostomy subjects, who consumed either a broccoli soup (with inactivated myrosinase) or a broccoli soup plus mustard seeds (containing an exogenous source of myrosinase to convert glucobrassicin into I3C), contained AhR ligands in amounts that were high enough to activate AhR in our reporter cell line (data not shown). Probably, the AhR ligands are either broken down, diluted too much or absorbed in the very first part of the duodenum. In order to rule out interference from the pancreatic juice on the AhR assay, we spiked one ileostomy sample with I3C which was then tested for AhR activation. Results showed that spiking an ileostomy sample with an amount of I3C, which produced a final concentration of $77 \mu \mathrm{M}$ in the AhR assay, led to an AhR activation that reached $57 \%$ of the positive control, while in the non-spiked sample only $1 \%$ AhR activation was found. In relation to Figure $2 \mathrm{~b}$, this implies that the extraction method is effective for $13 \mathrm{C}$ and besides, if there had been any AhR ligand present in the ileostomy samples, there would have been an AhR response. Indole AhR ligands were detected in the ileal fluid samples (Table 2), but I3C was only found in very limited amount (ranging from 0 - $71 \mathrm{ng} / \mathrm{g}$ wet weight ileal fluid, data not shown) and DIM was not detected at all. Although DIM was not detected, we did detect other indole derivatives. Kynurenine is a product of endogenous tryptophan metabolism, while the others (tryptamine, indole-3-aldehyde, indole-3-acetic acid and indole-3-lactic acid) are reported as products of bacterial tryptophan metabolism and possibly produced by the ileal microbiota from dietary tryptophan ${ }^{47}$. However, their concentrations were too low to trigger a significant AhR activation in our reporter assay ${ }^{48}$.

Overall, the data from the ileostomy study indicate that the concentration of indole derivatives arriving in the ileum level after broccoli soup consumption was too low to produce a significant increase in the AhR activation with the in vitro assay used herein. This may be due to a limited formation of AhR ligands from glucobrassicin or their absorption through the small intestinal epithelium 8.49 . It is possible that after preparation of the broccoli soup there is not enough glucobrassicin present to produce a measurable AhR activation, or that the exogenous myrosinase activity provided with mustard seeds was insufficient to hydrolyse glucobrassicin efficiently. It must be considered that glucosinolates can also reach the colon intact as reported by Maskell (1990) ${ }^{50}$. Elfoul et al., (2001) reported that $55 \%$ of the initial dose of glucosinolates was still intact in the colon of rats ${ }^{51}$. The role played by intact glucobrassicin reaching the colon is not known. Microbiota present in the colon can hydrolyse glucosinolates with a myrosinase activity which is about $10 \%$ of the activity present in broccoli, possibly resulting in AhR ligand formation ${ }^{8,41,52,53}$. The formation of sulforaphane from glucoraphanin by colonic microbiota 
has been demonstrated, but not the formation of I3C or its further cyclization into AhR ligands ${ }^{53}$. Therefore, it might be that the broccoli particles entering the colon, still containing glucosinolates, can be converted into AhR ligands by colonic microbiota and generate AhR activation in the slightly acidic conditions of the ascending colon. Nonetheless, AhR is highly expressed in, for example, intraepithelial lymphocytes and T-helper 17 cells, which are more abundant in the small intestine ${ }^{6,54}$. Therefore, it is important that most AhR ligands at least reach the small intestine, as no AhR are present in the stomach. In this in vitro study, we show that there was AhR activation after the gastric phase, indicating the presence of AhR ligands available for the small intestine. After the small intestine however, in both the ileostomy and in vitro samples, no AhR activation was measured anymore and the fate of AhR ligands and reason for lack of activity in the small intestine unfortunately remain unknown.

Table 2. Indole derivatives detected by LC-MS/MS in freeze-dried ileal fluid samples after subjects consumed either a broccoli soup or a broccoli soup + mustard seeds (200 mL). T1 is before ingestion and $\mathrm{T} 2$ is 4 hours after ingestion. $n=8$ donors. Data is expressed as $\mu \mathrm{g}$ or $n g$ per $\mathrm{g}$ dry weight ileostomy sample \pm SEM.

\begin{tabular}{|c|c|c|c|c|c|c|c|c|}
\hline \multirow[b]{3}{*}{ Tryptophan ( $\mu \mathrm{g})$} & \multicolumn{4}{|c|}{ Broccoli soup } & \multicolumn{4}{|c|}{ Broccoli soup + Mustard } \\
\hline & \multicolumn{2}{|c|}{$\mathrm{T} 1$} & \multicolumn{2}{|c|}{$\mathrm{T} 2$} & \multicolumn{2}{|c|}{ T1 } & \multicolumn{2}{|c|}{ T2 } \\
\hline & $60.8 \pm$ & 3.3 & $117.5 \pm$ & 23.2 & $79.2 \pm$ & 9.9 & $133.7 \pm$ & 18.0 \\
\hline Kynurenine (ng) & $87.6 \pm$ & 29.5 & $99.5 \pm$ & 17.7 & $67.8 \pm$ & 24.2 & $42.8 \pm$ & 11.5 \\
\hline Tryptamine (ng) & $155.8 \pm$ & 96.5 & $11.7 \pm$ & 5.2 & $111.6 \pm$ & 87.0 & $13.2 \pm$ & 4.6 \\
\hline Indole-3-lactic acid (ng) & $412.5 \pm$ & 150.5 & $88.6 \pm$ & 9.9 & $1098.6 \pm$ & 848.1 & $308.8 \pm$ & 99.6 \\
\hline Indole-3-aldehyde (ng) & $13.0 \pm$ & 8.4 & $34.4 \pm$ & 12.1 & $21.5 \pm$ & 15.1 & $103.9 \pm$ & 12.8 \\
\hline Indole-3-acetic acid (ng) & $50.9 \pm$ & 30.7 & $18.2 \pm$ & 12.6 & $87.8 \pm$ & 58.5 & $28.0 \pm$ & 17.5 \\
\hline
\end{tabular}




\section{Concluding remarks}

This research showed that activation of AhR from consumption of broccoli can be modulated by the thermal treatments during domestic cooking or industrial processing. In general, cooking broccoli decreased AhR activation and raw broccoli showed the highest AhR activation. However, a thermal treatment is not necessarily deleterious for the generation of AhR ligands, because mild thermal treatments, like steaming, preserve glucobrassicin and myrosinase activity. Furthermore, AhR activation after the gastric phase was higher for raw broccoli but not for the steamed or boiled broccoli, and pH in the gastric phase had no significant effect on AhR activation. However, no AhR activation was found after in vitro digestion or in ileal fluid samples from ileostomy subjects after consumption of broccoli soup, which could be due to processing of the broccoli, uptake of all AhR ligands in the small intestine or interference with the digestive enzymes used in the in vitro assay. Overall, it can be concluded that broccoli consumption resulted in AhR activation after the oral and gastric phase, with the highest AhR activation measured using raw broccoli. In the ileal fluid samples, the concentration of AhR ligands at ileum level was too low to induce significant AhR activation on our cell-assay, but production of AhR ligands in the stomach and their absorption by the small intestine epithelium could have beneficial effects on health.

\section{Acknowledgements}

Research presented in this publication was financially supported by the Graduate School VLAG.

\section{Conflicts of interest}

The authors declare no competing interests. 


\section{References}

1 Kapusta-Duch, J., Kopec, A., Piatkowska, E., Borczak, B. \& Leszczynska, T. The beneficial effects of Brassica vegetables on human health. Roczniki Państwowego Zakładu Higieny 63, (2012).

2 Kaczmarek, J. L. et al. Broccoli consumption affects the human gastrointestinal microbiota. The Journal of Nutritional Biochemistry 63, 27-34, (2019).

3 Hubbard, T. D. et al. Dietary broccoli impacts microbial community structure and attenuates chemically induced colitis in mice in an Ah receptor dependent manner. Journal of Functional Foods 37, 685-698, (2017).

$4 \mathrm{Li}$, Y. et al. Exogenous stimuli maintain intraepithelial lymphocytes via aryl hydrocarbon receptor activation. Cell 147, 629-640, (2011).

5 Hooper, L. V. You AhR what you eat: linking diet and immunity. Cell 147, 489-491, (2011).

6 Qiu, J. et al. The aryl hydrocarbon receptor regulates gut immunity through modulation of innate lymphoid cells. Immunity 36, 92-104, (2012).

7 Fahey, J. W., Zalcmann, A. T. \& Talalay, P. The chemical diversity and distribution of glucosinolates and isothiocyanates among plants. Phytochemistry 56, 5-51, (2001).

8 Holst, B. \& Williamson, G. A critical review of the bioavailability of glucosinolates and related compounds. Natural Product Reports 21, 425-447, (2004).

9 Kissen, R., Rossiter, J. T. \& Bones, A. M. in Phytochemistry Reviews Vol. 8 69-86 (2009).

10 Higdon, J., Drake, V. J. \& Williams, D. E. Indole-3-carbinol. Linus Pauling Institute, Oregon State University. [Online]. Available: http://lpi. oregonstate. edu/infocenter/phytochemicals/i3c/[December 2008], (2008).

11 Bjeldanes, L. F., Kim, J.Y., Grose, K. R., Bartholomew, J. C. \& Bradfield, C. A. Aromatic hydrocarbon responsiveness-receptor agonists generated from indole-3-carbinol in vitro and in vivo: comparisons with 2, 3, 7, 8-tetrachlorodibenzo-p-dioxin. Proceedings of the National Academy of Sciences 88, 95439547, (1991).

12 Matusheski, N. V. et al. Epithiospecifier protein from broccoli (Brassica oleracea L. ssp. italica) inhibits formation of the anticancer agent sulforaphane. Journal of Agricultural and Food Chemistry 54, $2069-$ 2076, (2006).

13 Aggarwal, B. B. \& Ichikawa, H. Molecular targets and anticancer potential of indole-3-carbinol and its derivatives. Cell Cycle 4, 1201-1215, (2005).

14 Nguyen, L. P. \& Bradfield, C. A. The search for endogenous activators of the aryl hydrocarbon receptor. Chemical Research in Toxicology 21, 102-116, (2007).

15 Jellinck, P. H. et al. Ah receptor binding properties of indole carbinols and induction of hepatic estradiol hydroxylation. Biochemical Pharmacology 45, 1129-1136, (1993).

16 Gil, V. \& MacLeod, A. The effects of $\mathrm{pH}$ on glucosinolate degradation by a thioglucoside glucohydrolase preparation. Phytochemistry 19, 2547-2551, (1980).

17 De Kruif, C. et al. Structure elucidation of acid reaction products of indole-3-carbinol: detection in vivo and enzyme induction in vitro. Chemico-Biological Interactions 80, 303-315, (1991).

18 Kong, F. \& Singh, R. Disintegration of solid foods in human stomach. Journal of Food Science 73, R67-R80, (2008).

19 Oliviero, T., Verkerk, R. \& Dekker, M. Effect of water content and temperature on glucosinolate degradation kinetics in broccoli (Brassica oleracea var. italica). Food Chemistry 132, 2037-2045, (2012).

20 Oerlemans, K., Barrett, D. M., Suades, C. B., Verkerk, R. \& Dekker, M. Thermal degradation of glucosinolates in red cabbage. Food Chemistry 95, 19-29, (2006). 
21 Minekus, M. et al. A standardised static in vitro digestion method suitable for food-an international consensus. Food \& function 5, 1113-1124, (2014).

22 McDougall, G. J. et al. Novel colon-available triterpenoids identified in raspberry fruits exhibit antigenotoxic activities in vitro. Molecular Nutrition \& Food Research 61, 1600327, (2017).

23 McDougall, G. J. et al. Tracking (Poly) phenol components from raspberries in ileal fluid. Journal of Agricultural and Food Chemistry 62, 7631-7641, (2014).

24 Dominguez-Perles, R. et al. A new ultra-rapid UHPLC/MS/MS method for assessing glucoraphanin and sulforaphane bioavailability in human urine. Food Chemistry 143, 132-138, (2014).

25 Fujioka, N. et al. Urinary 3, 3'-diindolylmethane: a biomarker of glucobrassicin exposure and indole-3carbinol uptake in humans. Cancer Epidemiology and Prevention Biomarkers 23, 282-287, (2014).

26 Wong, C. P. et al. Effects of sulforaphane and 3, 3'-diindolylmethane on genome-wide promoter methylation in normal prostate epithelial cells and prostate cancer cells. PloS one $\mathbf{9}$, e86787, (2014).

27 Vallejo, F., Tomás-Barberán, F. \& García-Viguera, C. Glucosinolates and vitamin C content in edible parts of broccoli florets after domestic cooking. European Food Research and Technology 215, 310-316, (2002).

28 Dekker, M., Verkerk, R. \& Jongen, W. M. Predictive modelling of health aspects in the food production chain: a case study on glucosinolates in cabbage. Trends in Food Science \& Technology 11, 174-181, (2000).

29 Song, L. \& Thornalley, P. J. Effect of storage, processing and cooking on glucosinolate content of Brassica vegetables. Food and Chemical Toxicology 45, 216-224, (2007).

30 Verkerk, R., Van der Gaag, M., Dekker, M. \& Jongen, W. Effects of processing conditions on glucosinolates in cruciferous vegetables. Cancer Letters 114, 193-194, (1997).

31 Rosa, E. A. \& Heaney, R. K. The effect of cooking and processing on the glucosinolate content: studies on four varieties of Portuguese cabbage and hybrid white cabbage. Journal of the Science of Food and Agriculture 62, 259-265, (1993).

32 Yuan, G.f., Sun, B., Yuan, J. \& Wang, Q.-m. Effects of different cooking methods on health-promoting compounds of broccoli. Journal of Zhejiang University Science B 10, 580, (2009).

33 Jones, R. B., Faragher, J. D. \& Winkler, S. A review of the influence of postharvest treatments on quality and glucosinolate content in broccoli (Brassica oleracea var. italica) heads. Postharvest Biology and Technology 41, 1-8, (2006).

34 Ciska, E. \& Kozłowska, H. The effect of cooking on the glucosinolates content in white cabbage. European Food Research and Technology 212, 582-587, (2001).

35 Verkerk, R. \& Dekker, M. Glucosinolates and myrosinase activity in red cabbage (Brassica oleracea L. var. Capitata f. rubra DC.) after various microwave treatments. Journal of Agricultural and Food Chemistry 52, 7318-7323, (2004).

36 Oliviero, T., Verkerk, R. \& Dekker, M. Isothiocyanates from Brassica Vegetables-Effects of Processing, Cooking, Mastication, and Digestion. Molecular Nutrition \& Food Research 62, 1701069, (2018).

37 Murray, I. A. \& Perdew, G. H. Ligand activation of the Ah receptor contributes to gastrointestinal homeostasis. Current opinion in toxicology 2, 15-23, (2017).

38 Hubbard, T. D., Murray, I. A. \& Perdew, G. H. Indole and Tryptophan Metabolism: Endogenous and Dietary Routes to Ah Receptor Activation. Drug Metabolism and Disposition 43, 1522-1535, (2015).

39 Rungapamestry, V., Duncan, A. J., Fuller, Z. \& Ratcliffe, B. Effect of cooking brassica vegetables on the subsequent hydrolysis and metabolic fate of glucosinolates. Proceedings of the Nutrition Society 66, 6981, (2007).

40 Burow, M. et al. ESP and ESM1 mediate indol-3-acetonitrile production from indol-3-ylmethyl glucosinolate in Arabidopsis. Phytochemistry 69, 663-671, (2008). 
41 Katz, E., Nisani, S. \& Chamovitz, D. A. Indole-3-carbinol: a plant hormone combatting cancer. F1000Research 7, (2018).

42 Howard, L. A., Jeffery, E. H., Wallig, M. A. \& Klein, B. P. Retention of phytochemicals in fresh and processed broccoli. Journal of Food Science 62, 1098-1104, (1997).

43 Mahn, A. \& Reyes, A. An overview of health-promoting compounds of broccoli (Brassica oleracea var. italica) and the effect of processing. Food Science and Technology International 18, 503-514, (2012).

44 Grose, K. R. \& Bjeldanes, L. F. Oligomerization of indole-3-carbinol in aqueous acid. Chemical Research in Toxicology 5, 188-193, (1992).

45 Uda, Y., Kurata, T. \& Arakawa, N. Effects of $\mathrm{pH}$ and ferrous ion on the degradation of glucosinolates by myrosinase. Agricultural and Biological Chemistry 50, 2735-2740, (1986).

46 Minekus, M. et al. A standardised static in vitro digestion method suitable for food-an international consensus. Food \& Function 5, 1113-1124, (2014).

47 Roager, H. M. \& Licht, T. R. Microbial tryptophan catabolites in health and disease. Nature Communications 9, 3294, (2018).

48 Koper, J. E. B. et al. Polyphenols and Tryptophan Metabolites Activate the Aryl Hydrocarbon Receptor in an in vitro Model of Colonic Fermentation. Molecular Nutrition \& Food Research 63, 1800722, (2019).

49 Patel, A. R., Spencer, S. D., Chougule, M. B., Safe, S. \& Singh, M. Pharmacokinetic evaluation and In VitroIn Vivo Correlation (IVIVC) of novel methylene-substituted 3, 3' diindolylmethane (DIM). European Journal of Pharmaceutical Sciences 46, 8-16, (2012).

50 Maskell, I. E. Nutritional aspects of feeding rapeseed products to rats and pigs. PhD dissertation, University of Newcastle upon Tyne, (1990).

51 Elfoul, L.etal. Formation of allyl isothiocyanate from sinigrin in the digestive tract of rats monoassociated with a human colonic strain of Bacteroides thetaiotaomicron. FEMS Microbiology Letters 197, 99-103, (2001).

52 Tian, S., Liu, X., Lei, P., Zhang, X. \& Shan, Y. Microbiota: a mediator to transform glucosinolate precursors in cruciferous vegetables to the active isothiocyanates. Journal of the Science of Food and Agriculture $\mathbf{9 8}$ 1255-1260, (2018).

53 Capuano, E., Dekker, M., Verkerk, R. \& Oliviero, T. Food as pharma? The case of glucosinolates. Current Pharmaceutical Design 23, 2697-2721, (2017).

54 Stockinger, B., Meglio, P. D., Gialitakis, M. \& Duarte, J. H. The aryl hydrocarbon receptor: multitasking in the immune system. Annual Review of Immunology 32, 403-432, (2014). 



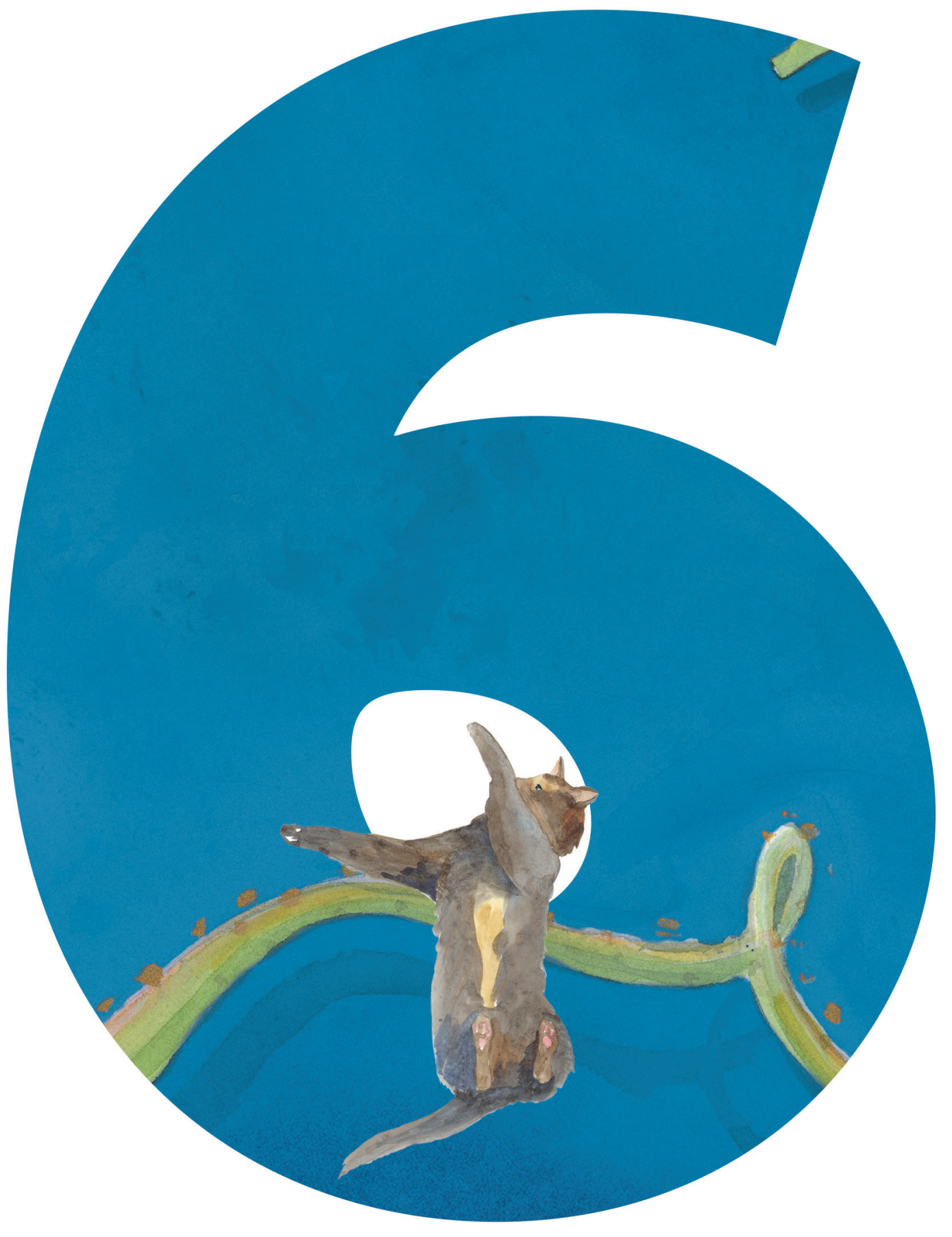




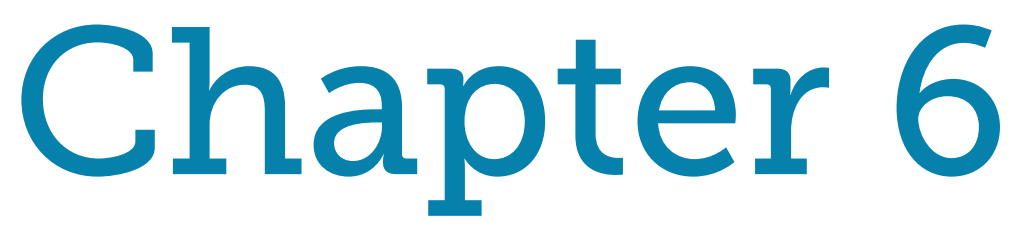

General discussion 



\section{The gut way to health}

This thesis entitled 'The gut way to health: in vitro studies on immunomodulatory food compounds', describes the relationship between diet, metabolites produced by the gut microbiota and the immune system, during in vitro digestion and fermentation. We aimed at highlighting the importance of the food matrix and the interaction with the microbiota in modulating the health effects of single food compounds, which is often neglegted ${ }^{1-7}$. By applying a unique approach using whole foods, rather than single components, we created a more physiologically relevant, but also a more complex model system. In this way, the role of the food matrix and the influence of food processing and fermentation or microbial metabolism is taken into account. This approach leads to more knowledge regarding the fate and immune-activating capacity of food compounds when consumed as part of a food product. Furthermore, starting from the obvious observation that dietary compounds can be transformed during their passage through the small or large intestine, we studied the fate of immuno-modulating compounds during digestion and how this modulates their biological activities on host cells in vitro. Our in vitro results can serve as a basis to design in vivo experiments by showing insights in the relationship between food, microbiota and the immune system.

We focused on both direct (by parent compounds) and indirect (by metabolites) immune-modulation of food. We investigated binding of dietary fibres on binding to dendritic cells and induced cytokine secretion, as well as ligand activation of the Aryl hydrocarbon Receptor (AhR), which is involved in the establishment and maintenance of intestinal homeostasis ${ }^{8-10}$. We hypothesized that food preparation, digestion and fermentation all have an effect on the capacity of immunomodulatory food compounds to modulate the immune system. Therefore, we ought to obtain more insight into the importance of food compounds during digestion and fermentation.

In Chapter 2, the fibre linear arabinan (LA), an oligosaccharide that is part of the complex structure of pectin, was shown to bind to dendritic cells and induce cytokine secretion through a calcium and spleen tyrosine kinase (SYK)-dependent mechanism. We ruled out interaction of LA with several C-type Lectin Receptors (CLR). Additionally, we showed that human colonic fermentation of natural plant matrices containing LA (apple and sugar beet) led to immunestimulatory activity.

In Chapter 3, oregano was selected as a source of polyphenols, and its potential in activating AhR during in vitro fermentation was studied. Fermentation of oregano released modest amounts of dietary polyphenols. The microbiota made a major contribution to AhR activation in the colon through the metabolism of tryptophan into indoles. Chapter $\mathbf{4}$ further explores the relationships between tryptophan supplementation, AhR activation and the evolution of tryptophan metabolites produced by gut microbiota. 
Finally, in Chapter $\mathbf{5}$ the contribution of another category of known AhR dietary ligands, i.e. glucosinolates, was studied. We specifically investigated the effects of different cooking methods of broccoli on AhR activation during in vitro and in vivo digestion. 


\section{Immune stimulation by food compounds}

The immune system is highly influenced by the intestinal microbiota ${ }^{11-13}$. Immune cells are known to bind parts of bacteria and viruses through pattern recognition receptors (PRRs), but they are also known to detect food compounds ${ }^{14}$. Dietary fibres, phytochemicals and microbial metabolites of tryptophan are gaining more attention due to their interaction with host receptors such as PRRs and AhR ${ }^{15-19}$. The relevance of these compounds is better understood in the small intestine compared to the colon, where microbial fermentation or metabolism can greatly influence their biological activities.

\subsection{Dietary fibres}

Non-digestible dietary fibres (DF) are fermented by the colonic microbiota resulting in the production of short-chain fatty acids (SCFA), mainly consisting of acetate, propionate and butyrate ${ }^{19}$. Butyrate has been shown to be beneficial for intestinal functioning, improvement of gut barrier function by stimulation of mucin formation, and it has anti-inflammatory effects ${ }^{20,21}$. Besides the indirect immunomodulatory effect exerted by fibres through the products of their fermentation (SCFAs), specific glycan structures can directly bind to immune cells. A typical example is fungal $\beta$-glucan, which was found to bind the CLR Dectin- ${ }^{22,23}$. Furthermore, it was recently shown that also other polysaccharides can induce cytokine production by binding to dendritic cells in mice ${ }^{19}$. It was recently reported that LA could activate mouse bone marrowderived dendritic cells from TLR2/4 knockout mice ${ }^{19}$, but LPS contamination prevented studies on human immune cells ${ }^{14}$. Therefore, in Chapter 2 we investigated the potential of linear arabinan (LA) from sugar beet to bind and activate human dendritic cells leading to cytokine secretion. By purifying LA free of Microbe-associated molecular patterns (MAMPs), e.g. lipopolysaccharide (LPS), we could demonstrate specific binding and activation of LA to human dendritic cells (DC). Immune activation of human DC was calcium-dependent and SYK-dependent, suggesting interaction of LA with a CLR containing an ITAM or hemi-ITAM motif. Experiments with siRNAs and blocking antibodies failed to identify a role for the most well-characterised ITAM-containing CLRs i.e. Mincle, DC-SIGN, Dectin-1 and Dectin-2. Identification of this receptor through reverse genetics or siRNA inhibition of other CLRs in the human genome will enable investigation of its possible expression in intestinal epithelial cells and different immune cells. Ultimately this will lead to more insights into the role of this receptor in human biology.

Furthermore, as LA does not occur in food as isolated compound but as part of the pectin backbone within a complex plant cell wall, we investigated the potential of LA-containing pectin and plant matrices (apple and sugar beet) to induce cytokine production during human in vitro fermentation. Fermentation of apple and sugar beet by human microbiota indeed led to immune activation of BMDCs from TLR4/TLR2 knockout mice. However, extracted sugar beet pectin did not induce immune activation of DCs, even when the arabinan was present in 
relatively higher amounts than in apple or sugar beet. Particulate LA induced a higher immune response by DCs than soluble LA ${ }^{19}$, which resulted in a lower cytokine production in our study by the use of supernatant. Investigating the bacterial pellet, including particulate arabinans, would therefore be an interesting addition to our study. In any case, these results indicate that the supramolecular organisation of the pectin molecule and the solubilisation of ligands from the matrix must be taken into account when judging the immunomodulatory effect of linear arabinans in real foods.

\subsection{Polyphenols}

In Chapter 3, we performed an intensive screening to test the capacity of isolated polyphenols to activate AhR in the Chemical Activated Luciferase gene eXpression (CALUX) assay. Several studies have reported conflicting results on the capacity of polyphenols to activate $A h R^{1,24-27}$, most likely due to the use of different cell types in the reporter assay ${ }^{28}$. Our results showed that luteolin, baicalein, and 4-O-caffeoylquinic acid dose-dependently activated $A h R$, while quercetin reduced AhR activation when presented to the cells in combination with luteolin, suggesting an antagonistic effect of quercetin on AhR activation. This indicates that the in vivo biological effect can be difficult to predict when using foods or whole diets, because polyphenols typically occur as complex mixtures of chemical structures at varying concentrations. Based on our screening we selected oregano, a rich source of luteolin, to study the effect of the food matrix on the fate of luteolin during in vitro fermentation and its capacity to activate AhR.

In the colon polyphenols might be directly absorbed or metabolised by bacteria into smaller compounds, typically phenyl acetic acid and phenyl propionic acid derivatives. Therefore, it is important to know the kinetics of polyphenol degradation in the colon, because this has an impact on the pharmacokinetics of their distribution and ultimately on their bioactivity. By using the Simulator of the Human Intestinal Microbial Ecosystem (SHIME) fermentation model, we were able to study the fate of polyphenolic AhR ligands in each part of the colon, which is not possible using a simpler in vitro batch fermentation system or through in vivo studies. Our results showed limited breakdown of luteolin in the ascending colon, whereas luteolin was completely converted to other metabolites by the microbiota in the transverse colon. This indicates that the microbiota in the ascending colon is unable to metabolise polyphenols, allowing them to remain in that part of the colon for a longer time. This longer time may be sufficient for transport in the ascending colon and AhR activation in the mucosa and other tissues.

We furthermore showed that oregano could increase AhR activation in the ascending colon, but the microbial derived tryptophan metabolites were more abundant than the polyphenols from oregano, clearly contributing majorly to AhR activation in the gut. There was a high AhR activating 
capacity by tryptophan metabolites, indicating that the relevance of polyphenols as AhR ligands may be rather limited. Furthermore, the in vivo biological effects will depend on transport across the colonic epithelium and this has yet to be studied for metabolites like polyphenols.

\subsection{Tryptophan metabolites}

To gain more insight into the potential of tryptophan metabolites to activate AhR, we studied tryptophan (Trp) and its derivatives during in vitro fermentation in Chapter 4. For the first time, an intestinal model of the human microbiota ecosystem has been used to investigate the effect of Trp supplementation on the profile of Trp metabolites generated in different parts of the colon. The duration of the fermentation experiment was 3 weeks, of which the first week was control feeding, followed by one week of Trp supplementation, and finally another one-week wash-out period with control feeding. Trp supplementation increased the amount of Trp metabolites in the fermenter supernatants. Most Trp metabolites were produced in the transcending colon (TC), where also the largest effect of Trp on the microbiota composition was observed. In the TC the relative abundance of AhR ligand producers Enterococcus faecalis, Lactobacillus reuteri and Lactobacillus murinus were increased as a result of Trp supplementation. Overall, these findings indicate that Trp supplementation can increase Trp metabolism and consequently the production of AhR agonists. However, when the AhR activation of the supernatants collected at different days of treatment was measured, the overall AhR activation of supernatants was lower during the Trp supplementation period than during the control period. We hypothesized that this was due to an antagonistic effect exerted by Trp, which does not activate AhR in our in vitro cell assay, and which concentration in the supernatants was highly negatively correlated to the AhR activation. This hypothesis was proven by measuring AhR activation of mixtures of Trp and tryptamine, where results showed that the presence of Trp reduced AhR activation induced by tryptamine. Again, this highlights the difficulty in predicting overall in vivo biological effects using isolated compounds rather than mixtures.

As only a small fraction of Trp is converted into AhR ligands, the presence of large amounts of Trp in the cellular environment may limit the efficacy of Trp metabolites in activating AhR. This depends on the relative transport of Trp through the colon epithelium compared to that of Trp metabolites. To investigate the transport of Trp and its derivative tryptamine (Trm) across the intestinal epithelium, we performed a pilot study using colonic pig organoids (Figure 1). The organoids were grown as polarised monolayers on Transwell filters according to the method of van der Hee et al., $2018^{29}$ and were stimulated with a mixture of Trp and Trm (both 20 HM) for 30 and 120 minutes at either the apical or basolateral side, and both apical and basolateral sides were sampled after incubation. The trans-epithelial resistance of the monolayers was monitored during the incubation to check for integrity of the monolayer as previously described ${ }^{29}$. Afterwards, the concentration of tryptophan and tryptamine were determined using LC/MSMS as described in Chapter 5. 
Apical stimulation $\mathbf{3 0} \mathrm{min}$

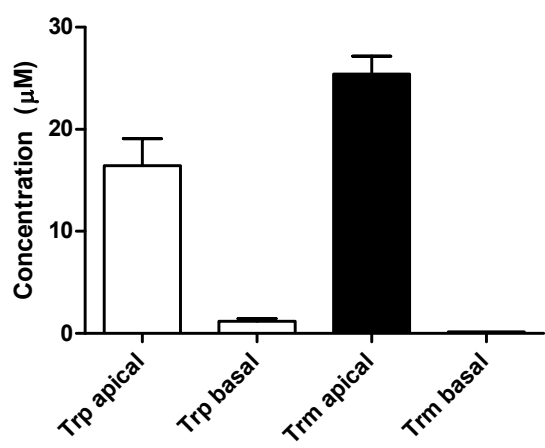

Basal stimulation $30 \mathrm{~min}$

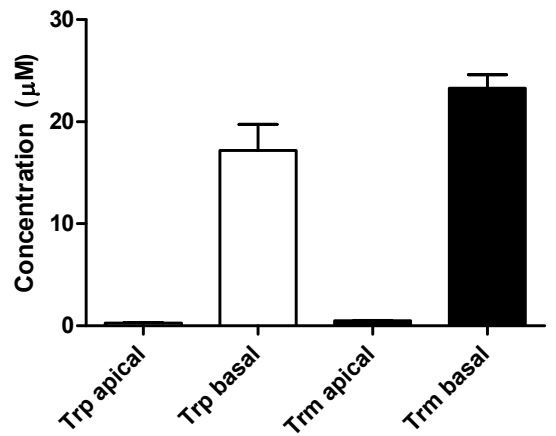

Apical stimulation $120 \mathrm{~min}$

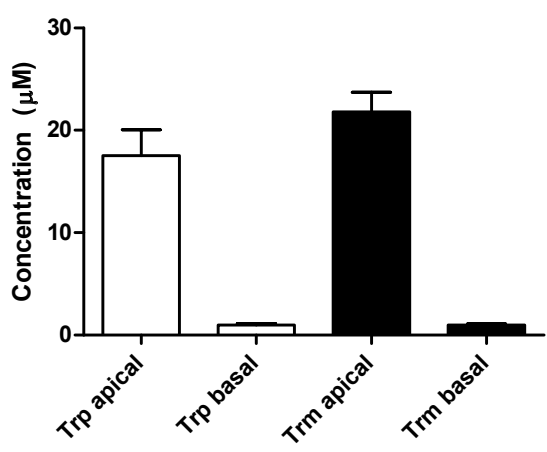

Basal stimulation 120 min

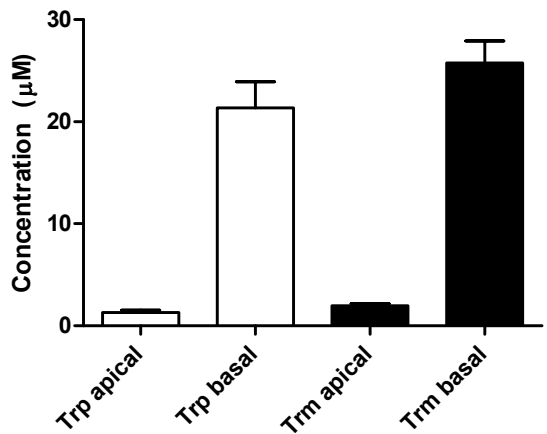

Figure 1. Preliminary results on trans-epithelial transport of tryptophan (Trp) and tryptamine (Trm) across an intact monolayer of colon organoid cells. Trp and Trm were added to either apical or basolateral compartments and samples from both compartments were taken after 30 and 120 minutes incubation. The concentrations of Trp and Trp were determined using LC-MS/MS.

The results show that Trp was transported from apical to basolateral side after already 30 minutes (around $7 \%$ ) while Trm transport occurred at a slower rate (4 \% after 120 min). Furtheremore, there was some transport from the basolateral side towards the apical side, so back into the lumen, of both Trp and Trm with respectively 1.5 and $2 \%$ transported after 30 min, and 6 and $7 \%$ transported after 120 min. Furthermore, the concentration of Trp transported from apical to basolateral side did not increase over time. This might be due to organoid metabolism, or uptake by the organoids without excretion to the basolateral side. Although the amounts transported in vitro seem relatively small, the total amounts transported in vivo would be substantial considering the surface area of the intestine. Combined with the 
previously found antagonistic effects of Trp on AhR, these data indicate that Trp and Trm can be absorbed by intestinal immune cells simultaneously, possibly at a similar ratio as observed in our in vitro fermentation system. Therefore, when aiming for in vivo AhR activation in the colon induced by AhR ligands like Trm, the balance between agonists and antagonists should be carefully determined.

Colonic protein fermentation is usually seen as detrimental for the host health ${ }^{30}$. However, as shown in our results, depending on the type of protein and metabolites formed, protein fermentation might also have beneficial effects on intestinal functioning. This also relates to the microbiota, since we showed that several beneficial species in the colon increased after Trp supplementation (Chapter 4). The recent findings that Trp metabolites can improve gut barrier function are in conflict with studies on protein fermentation and increased inflammatory responses (Figure 2 ) $^{31}$. There must be a paradigm shift when it comes to the effects of protein fermentation in the colon towards a re-evaluation of the physiological relevance of protein metabolites on host health. Furthermore, the role of protein fermentation in colonic health may be confounded by the harmful effects exerted by other compounds found in e.g. grilled or processed meat.

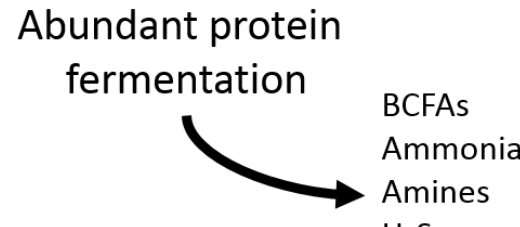

$\mathrm{H}_{2} \mathrm{~S}$

P-cresol
Fibre fermentation Trp fermentation?

SCFAs

Trp metabolites

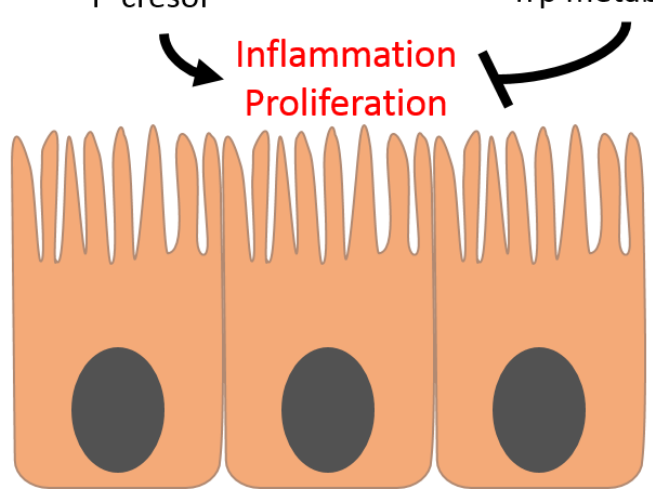

Figure 2. Abundant proteolytic fermentation generates compounds that may promote inflammatory responses and proliferation of colonocytes, whereas fibre fermentation and short-chain fatty acid production together with tryptophan metabolism have a protective role. Adjusted from Diether et al. ${ }^{31}$. 


\subsection{Glucosinolates and AhR activation}

In Chapter 5, we tested another class of dietary phytochemicals that are reported to be precursors of AhR ligands, namely glucosinolates and in particular, glucobrassicin, of which broccoli is a very rich source. We first tested the glucobrassicin derivatives 3,3'-Diindolylmethane (DIM) and Indole-3-Carbinol (I3C), using HepG2-Lucia ${ }^{\text {TM }}$ AhR reporter cells, and we found that DIM induced a higher AhR activation than I3C. This confirms that DIM is a more potent AhR ligand, which was confirmed by other studies ${ }^{18,32}$. However, the magnitude of AhR activation measured with the HepG2-Lucia ${ }^{\text {TM }}$ AhR cell line was different compared to what has been reported with other assays.

We tested the effect of different cooking methods on the capacity of broccoli to activate AhR. Several factors affect AhR activation by glucosinolate derivatives present in broccoli, including cooking method, cooking time and digestion. In particular the way broccoli is processed at industrial or, more frequently, domestic scale, can affect the residual myrosinase activity, which is crucial for the breakdown of glucobrassicin into the breakdown products I3C and DIM. This obvious notion has not yet been explored in the scientific literature despite the interest in the health effects of broccoli and its potential to activate AhR. Broccoli consumption resulted in AhR activation after the oral and gastric phase in vitro (Chapter 5). In general, raw broccoli showed the highest AhR activation and cooking broccoli decreased AhR activation. However, a thermal treatment is not necessarily deleterious for the generation of AhR ligands, because mild thermal treatments, such as steaming, preserves glucobrassicin and myrosinase activity (Chapter 5). Furthermore, we showed that the breakdown products of glucobrassicin, i.e. I3C, was only present in very small amounts and DIM was not present in ileostomy samples of patients that received a broccoli soup, even after addition of an exogenous source of myrosinase. This suggests that the way broccoli is processed is highly important for the magnitude of its effect on AhR activation. The fact that glucosinolate breakdown products do not reach the colon may depend on their absorption during the passage through the small intestine. A fraction of intact glucosinolates do reach the colon and is metabolised by the microbiota ${ }^{33}$. Whereas it has been shown that the gut microbiota possesses myrosinase-like activity and that it can produce sulforaphane from glucoraphanin, the hydrolysis of glucobrassicin and potential production of AhR ligands in the large intestine has not yet been proven. Therefore, the relevance of dietary glucosinolates to AhR activation in the gut may strongly depend on the way food is processed. 


\section{Methodological aspects}

\subsection{AhR activation measurements in cell cultures}

To measure AhR activation, three methods were used in this thesis: the CALUX assay (Chapter 3 and 4), qPCR measuring gene expression (Chapter 3), and the recently developed human HepG2-Lucia' ${ }^{\text {TM }}$ AhR reporter cells (Chapter 5). In literature, many different methods to measure AhR activation are used, such as in vitro cell assays and in vivo experiments using mice ${ }^{34-36}$. We can argue what the best method is, considering the various outcomes of similar compounds when different methods are used. The CALUX assay has the advantage of being a very stable cell line, and can be used as a high throughput method. The recently developed HepG2-Lucia AhR reporter cells could also be used as high throughput. However, this cell line has the practical disadvantage that the quality is only guaranteed for 20 passages by the company (Invivogen), whereas the CALUX cell line cells can be passaged up to 200 times. The main difference between the CALUX and the HepG2-Lucia AhR reporter cells is the origin of the cells. The CALUX cells used in this thesis originate from rat liver cells, whereas the Lucia cells originate from human liver cells. Both cell lines originate from the liver, and even though we study colonic samples, we did not choose to use the human HT29-Lucia-AhR (colonic cells) cells because these might also express drug transporter proteins originating from the small intestinal epithelia. In order to avoid interference with drug transporters, we used the liver cells to study AhR activating capacity by ligands.

It is known that rodent AhR respond differently to the same ligands compared to the human AhR, although in general AhR is evolutionary conserved across species ${ }^{37-41}$. For example, indole derivatives bind with a higher affinity to human AhR than mice $A h R^{40}$. In Chapters 3 and 4 we used the CALUX assay, thus rat liver cells. Therefore, AhR activation induced by tryptophan derivatives in humans might be higher than we reported. In Chapter 5, in which we studied the indole derivatives from glucobrassicin, we used human HepG2-Lucia cells, and indeed we found a higher AhR activation induced by DIM than in the CALUX assay ( $p<0.0001$; Figure 3a). In contrast, dioxins like 2,3,7,8-tetrachloordibenzo-p-dioxin (TCDD) have a 10-fold higher affinity for mice AhR compared to human $\mathrm{AhR}^{41}$.

Also $\beta$-naphthoflavone (positive control used for the AhR activation measurements) gives a different relative light unit (RLU) value for both reporter cell lines, even 1.5 times higher in the CALUX assay ( $p<0.0001$; Figure 3b). The AhR activation in this thesis was expressed as a percentage of the positive control and therefore, it becomes difficult to compare the results from the different studies. It is important to put results into perspective of its cell type origin and to use the activation results only as an indication of which compounds activate AhR and which are more or less potent in a cell type of specific origin. In order to compare the outcomes from different labs, there is need for a standardisation of the 
methods. Furthermore, studies that use rodents as a model to measure AhR activation in human should be carefully interpreted and human studies (in vivo or at least ex vivo) are necessary to confirm results.
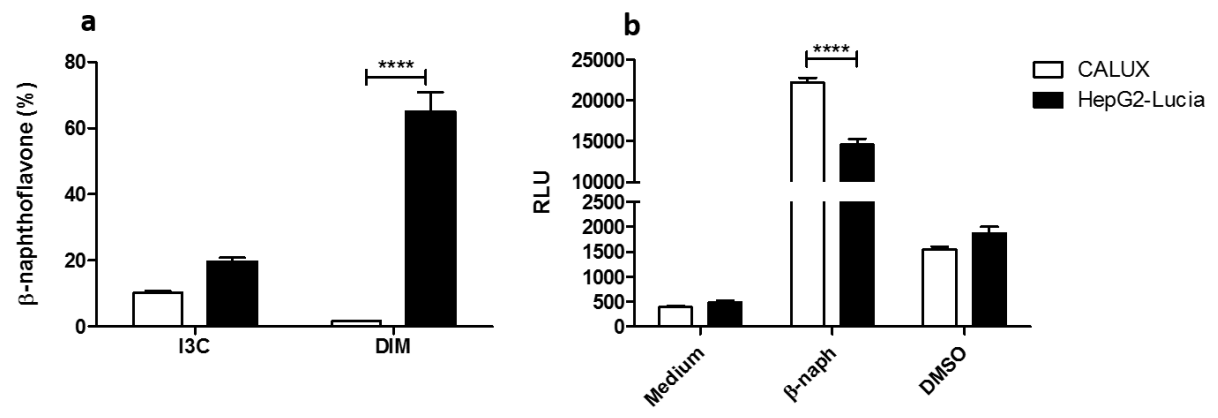

Figure 3. AhR activation using two different cell assays: the Chemical Activated Luciferase gene eXpression (CALUX, white) and the HepG2-Lucia AhR reporter cells (black). a) expressed as percent of the positive control $\beta$-naphthoflavone ( $5 \mu \mathrm{M})$ for indole-3-carbinol (I3C) and 3,3'-Diindolylmethane (DIM), $n=3$ and b) expressed as relative light units (RLU), medium, $1 \%$ DMSO in medium, and $5 \mu \mathrm{M}$ $\beta$-naphthoflavone ( $\beta$-naph) using $n=6 .{ }^{* * *} p<0.0001$, ANOVA with Bonferroni post-hoc test.

\subsection{Digestion studies}

In this thesis, we used an in vitro approach to get a mechanistic understanding of processes in the body. This would not be possible using in vivo studies, because of limitations in sampling location and ethical concerns. Naturally, it is challenging to simulate the highly complex physiological and chemical processes of the human body ${ }^{42}$, but in vitro models are developed to reflect the in vivo conditions as much as possible.

In order to compare results obtained in different labs across the globe, an in vitro small intestinal digestion method was developed, called INFOGEST ${ }^{43}$. This method is claimed to reflect the in vivo digestion conditions better than other methods, and it includes all steps starting from the oral cavity to the small intestinal phase. When using an in vitro digestion model, inter-individual differences are eliminated. This has the advantage that there is less variability between replicates and differences due to treatments are easier to interpret and normally less replicates are needed than in vivo. Inter-individual differences exist in, for example, isothiocyanate absorption, chewing time and therefore the amount of saliva, and enzyme activity ${ }^{44-46}$. These individual differences were taken into account in the ileostomy study (Chapter 5), as we used multiple subjects. All in all, there is need to validate the in vitro models with in vivo data, which has been done only to a limited extent with the INFOGEST protocol. 
Although the INFOGEST in vitro digestion method has the advantages described above (used in Chapter 5), there are also some limitations. Firstly, the method is not yet validated for specific food matrices and some compounds, and some parameters might be different in vivo, depending on the amount and mixture with other foods. Furthermore, due to the static nature of the method, it does not reproduce the dynamic changes in some physiological conditions in the small intestine, like the gradual change of $\mathrm{pH}$ in the gastric compartment, the gradual emptying of the stomach, not to mention the actual mixing and flowing regime as in vivo. There is also a lack of chewing during the oral phase of the INFOGEST method. Instead of chewing, milling was performed in our study (Chapter 5), which might result in an increased surface area, thereby possibly changing the glucosinolate hydrolysis ${ }^{47}$. Additionally, the oral phase takes longer during in vitro digestion than during in vivo digestion, which could also result in increased glucosinolate hydrolysis ${ }^{43}$. Studies that make use of ileostomy subjects as we described in Chapter 5, could provide us with some answers regarding inter-individual differences. However, as mentioned before, human intervention studies are costly, labour intensive and have ethical constraints. Human interventions should be carefully designed, taking into account intestinal passage time and inter-individual variation therein, as well as the initial dosage and the limit of detection of the techniques used for the quantification of the relevant biomarkers (e.g. HPLC/MS-MS).

\subsection{Fermentation studies}

In the other chapters in this thesis, we used simulated in vitro fermentation methods. The advantage of using such in vitro models is that the system is automated so that the changes in microbial population and metabolism can be studied upon long-term feeding. Furthermore, the colon is divided into 3 parts, each with a distinctive microbiota so that the metabolism dynamics in these 3 macro-areas can be studied. The use of the SHIME has allowed us to understand the complex dynamics of formation of immunomodulating food compounds during fermentation in the three separate colon parts, such as LA in sugar beet and apple, polyphenols from oregano and tryptophan with its metabolites. These results would have been impossible to obtain with in vivo studies or only partially possible with other (semi)-dynamic systems (e.g. TIM-2). However, it is questionable whether the same dynamics of the metabolites evolution is observed in vivo.

The in vitro fermentation model used in this thesis also has some limitations. First of all, the SHIME is semi-dynamic fermentation, i.e. batches in series rather than plug-flow. Furthermore, under its conventional set-up, the system cannot reproduce absorption in the colon. When metabolites are not removed by the system, the dynamics of metabolite interconversion can be biased and the accumulation of e.g. SCFA may change the microbial metabolism. This limitation can be partly overcome with a system where dialysis is implemented ${ }^{48}$. In addition to the lack of absorption in the colon, the lack of an absorption step in the small intestinal phase of the in vitro system must be considered to realistically reproduce what enters the colon after digestion of real diets. This is of course strongly dependent on the type of compounds that are studied and 
is, for example, of little importance for polyphenols (limited absorption in the small intestine) but very important for Trp (almost completely absorbed in the small intestine). In our studies, we fed the microbiota pre-digested material, which must also be considered in other studies.

Another point of discussion is whether or not to pool the faecal inoculum used for in vitro fermentation studies ${ }^{49}$. The microbial community is a complex mixture of many species with high inter-individual variation and possibly distinct enterotypes ${ }^{50,51}$. Microbes will compete with each other until a microbial balance is settled. This microbial balance can be disturbed by various factors, such as pathogens and dietary changes. It is debatable how many individual faecal samples should be mixed when pooling. Furthermore, even when an average microbiota is reached ${ }^{49}$, this might still represent only one (other) individual. Therefore, we decided to use individual faecal samples rather than to pool them, as also recommended by the SHIME manufacturer ProDigest ${ }^{48}$. However, pooling has the advantage that experiments can be repeated using the same microbial subset, but also the individual inoculum can be frozen ${ }^{52}$, thereby facilitating repetition possibilities.

In our fermentation studies, we observed large inter-individual microbiota variation and different changes in species after tryptophan supplementation (Chapter 4). However, AhR activation induced by fermenter supernatants did not vary much between donors (Chapters 3 and 4). This implies that, even though individuals in our study had different microbiota, the functionality linked to AhR activation was similar. A similar function of different microbiota is also proven in other studies where a functional overlap of the microbiota of different individuals was found, probably due to metabolic cross-feeding ${ }^{53-56}$. As a result, different individuals might have a comparable benefit from certain bioactives, even though they might have a substantially different microbiota composition. It must be noted though, that our fermentation protocol comprises a stabilization period of 2 weeks before the start of the experiments and during this period microbiota from different donors are fed with a standardized diet. This may have reduced the inter-individual variation in the microbiota (Chapters 3 and 4). 


\section{Future perspectives}

Possible avenues for continuing this research are shown in Figure 4. We studied the capacity of LA to bind human DCs, thereby aiming to obtain more information regarding the human relevance over mice. LA showed to induce cytokine production in human DCs, however, it still remains unknown whether the optimal effect can only be produced in vivo by purified LA preparations or whether the same effect can be obtained from plant sources of LA and pectin. Furthermore, the relative contribution on immune activation by direct binding to immune cells by DFs next to the production of SCFAs should be evaluated. It is important to identify the receptor involved in the arabinan binding to DCs as it can provide new insights into the function of this receptor in humans. Possible approaches are immune-precipitation of the receptor with LA antibody and its detection by proteomics, or studies of receptor-LA binding in the Biacore apparatus. In a preliminary study, we bound C-type lectin fusion proteins made by Mayer et al. ${ }^{57}$ on a G-protein chip, after which we used the Biacore to measure potential binding of LA to any of the C-type lectin fusion proteins. Follow-up studies are needed because so far we only observed weak non-specific binding.

The relevance of AhR activation in humans has not been studied to date and should be carried out in the future. Studying the binding of potential AhR ligands to human cells in vitro, unfortunately does not gives information of its relevance in vivo. Using in vitro studies, both AhR binding assays and digestion/fermentation studies, a selection of compounds of interest should be made and the results can serve as a base for in vivo trials. Based on our results, phytochemicals like polyphenols and glucobrassicin are potent AhR ligands, but these should be provided in relatively high amounts to see an effect on AhR activation. It would be interesting to study a mixture of several AhR ligands from phytochemicals, which could be present in a normal diet. Such studies might explain the fact that oregano supplementation resulted in increased AhR activation in the ascending colon, while the AhR ligand luteolin was present in amounts that were too low to activate AhR.

Furthermore, researching the capacity of gut microbiota to produce AhR ligands from metabolism of I3C, DIM and ICZ from intact glucosinolates can give insight in their relevance for colonic functioning and AhR activation. A first start would be the supplementation of glucobrassicin to the gut microbiota in vitro, followed by measurements of possible production of I3C and DIM. To make the study more realistic, in vitro digested broccoli could be added to an in vitro fermenter or, possibly more relevant, samples from ileal subjects who received a relatively high dose of cooked broccoli in a meal could be added to the colonic fermenter. Furthermore, an in vivo intervention with differently cooked broccoli, also including raw broccoli, may validate our findings and clarify the role of thermal treatments on AhR activation in the human intestine. The major difficulty is how to measure AhR activation in vivo ${ }^{58}$. Either ex vivo AhR measurements, or obtaining biopic 
material from colonic cells after which gene expression is measured, are probably reliable, but invasive options ${ }^{58}$.

A more potent way to increase AhR activation could be supplementation of Trp, which showed to increase the amount of AhR ligands produced in the colon. As we showed that excessive Trp might play an antagonistic role regarding AhR activation (Chapter 4), an in vivo study using AhR active Trp derivatives is worth investigating in the future. It is not clear whether we can modulate the amount of AhR ligands from Trp through the diet or food design, or by e.g. microbial transplantation. Future research could focus on the role of food design on the formation of immunomodulatory food compounds and their place of action in the gut. If Trp is proven to have beneficial effect in humans in vivo, the role of protein fermentation on human health should be re-evaluated. A schematic overview of the possible future research directions is presented in Figure 4. The results in this thesis gave us more insight towards the gut way to health.

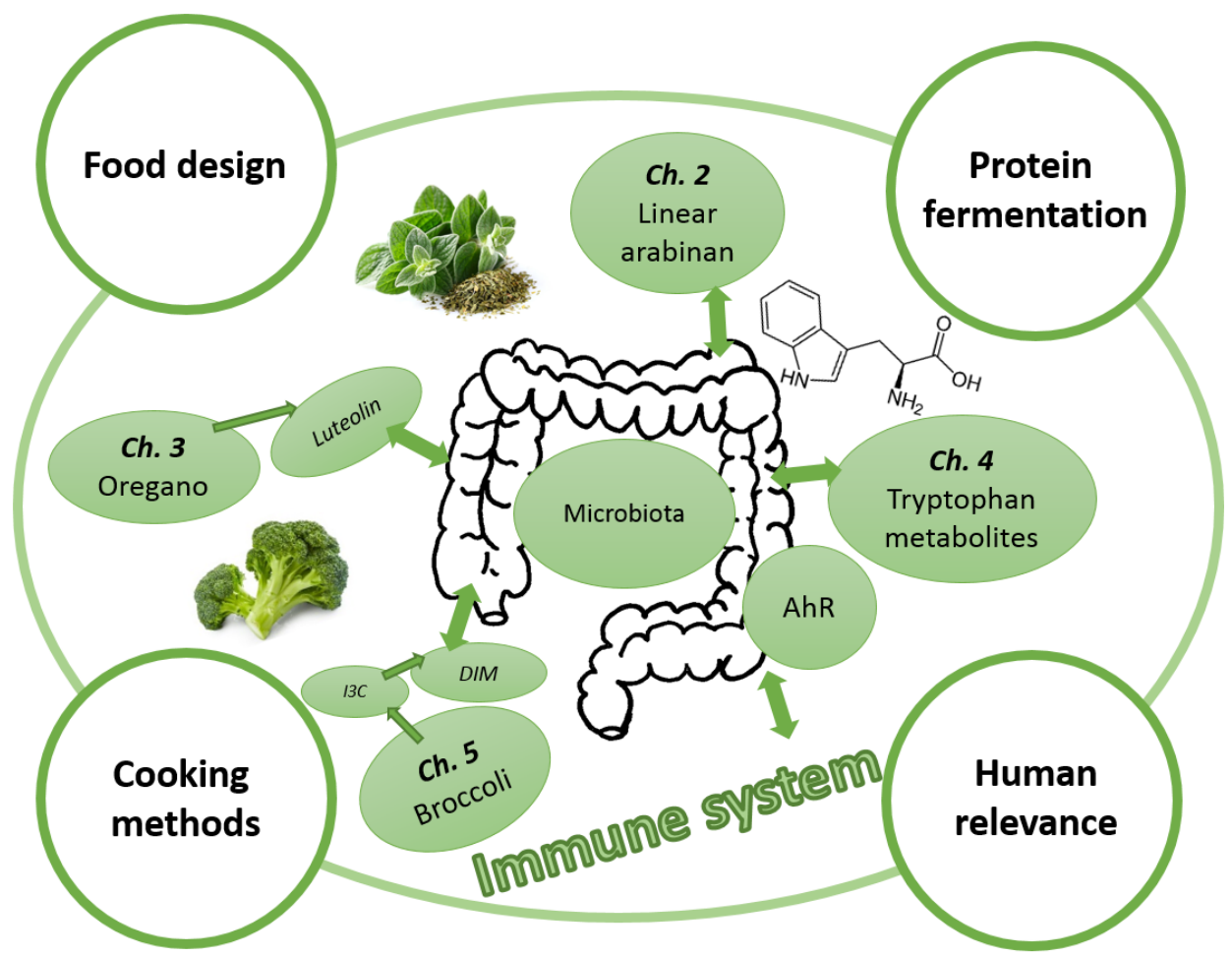

Figure 4. Schematic overview of the thesis with future research directions: food design, cooking methods, protein fermentation and human relevance. 


\section{References}

1 Amakura, Y. et al. Influence of food polyphenols on aryl hydrocarbon receptor-signaling pathway estimated by in vitro bioassay. Phytochemistry 69, 3117-3130, (2008).

2 Denison, M. S., Pandini, A., Nagy, S. R., Baldwin, E. P. \& Bonati, L. Ligand binding and activation of the Ah receptor. Chemico-biological interactions 141, 3-24, (2002).

3 Fukuda, I. et al. Pigments in green tea leaves (Camellia sinensis) suppress transformation of the aryl hydrocarbon receptor induced by dioxin. Journal of Agricultural and Food Chemistry 52, 2499-2506, (2004).

4 Bonnesen, C., Eggleston, I. M. \& Hayes, J. D. Dietary Indoles and Isothiocyanates That Are Generated from Cruciferous Vegetables Can Both Stimulate Apoptosis and Confer Protection against DNA Damage in Human Colon Cell Lines. Cancer Research 61, 6120-6130, (2001).

5 Busbee, P. B., Nagarkatti, M. \& Nagarkatti, P. S. Natural indoles, indole-3-carbinol (I3C) and 3,3'-diindolylmethane (DIM), attenuate staphylococcal enterotoxin B-mediated liver injury by downregulating miR-31 expression and promoting caspase-2-mediated apoptosis. PLoS One 10, e0118506, (2015).

6 Parada, J. \& Aguilera, J. Food microstructure affects the bioavailability of several nutrients. Journal of Food Science 72, R21-R32, (2007).

7 Fardet, A. Food health potential is primarily due to its matrix structure, then nutrient composition: A new paradigm for food classification according to technological processes applied. Journal of Nutritional Health \& Food Engineering 1, 31, (2014).

8 Lee, J. S. et al. AHR drives the development of gut ILC22 cells and postnatal lymphoid tissues via pathways dependent on and independent of Notch. Nature Immunology 13, 144, (2012).

9 Li, Y. et al. Exogenous stimuli maintain intraepithelial lymphocytes via aryl hydrocarbon receptor activation. Cell 147, 629-640, (2011).

10 Zelante, T.et al. Tryptophan catabolites from microbiota engage aryl hydrocarbon receptor and balance mucosal reactivity via interleukin-22. Immunity 39, 372-385, (2013).

11 Agus, A., Planchais, J. \& Sokol, H. Gut microbiota regulation of tryptophan metabolism in health and disease. Cell host \& microbe 23, 716-724, (2018).

12 Lazar, $V$. et al. Aspects of gut microbiota and immune system interactions in infectious diseases, immunopathology and cancer. Frontiers in Immunology 9, 1830, (2018).

13 Wells, J. M., Rossi, O., Meijerink, M. \& van Baarlen, P. Epithelial crosstalk at the microbiota-mucosal interface. Proceedings of the National Academy of Sciences of the United States of America 108 Suppl 1 , 4607-4614, (2011).

14 Govers, C. et al. Lipopolysaccharide quantification and alkali-based inactivation in polysaccharide preparations to enable in vitro immune modulatory studies. Bioactive Carbohydrates and Dietary Fibre 8, 15-25, (2016).

15 Wells, J. M.etal. Homeostasis of the gut barrier and potential biomarkers. American Journal of PhysiologyGastrointestinal and Liver Physiology 312, G171-G193, (2016).

16 Geijtenbeek, T. B. H. \& Gringhuis, S. I. Signalling through C-type lectin receptors: shaping immune responses. Nature Reviews Immunology $\mathbf{9}, 465$, (2009).

17 Sun, M., Ma, N., He, T., Johnston, L. J. \& Ma, X. Tryptophan (Trp) modulates gut homeostasis via aryl hydrocarbon receptor (AhR). Critical Reviews in Food Science and Nutrition, 1-9, (2019). 
18 Hubbard, T. D. et al. Dietary broccoli impacts microbial community structure and attenuates chemically induced colitis in mice in an Ah receptor dependent manner. Journal of Functional Foods 37, 685-698, (2017).

19 Meijerink, M.et al. Structure dependent-immunomodulation by sugar beet arabinans via a SYK tyrosine kinase-dependent signalling pathway. Frontiers in Immunology 9, 1972, (2018).

20 Kaur, A., Rose, D. J., Rumpagaporn, P., Patterson, J. A. \& Hamaker, B. R. In vitro batch fecal fermentation comparison of gas and short-chain fatty acid production using "slowly fermentable" dietary fibers. Journal of Food Science 76, H137-H142, (2011).

21 Rivière, A., Selak, M., Lantin, D., Leroy, F. \& De Vuyst, L. Bifidobacteria and Butyrate-Producing Colon Bacteria: Importance and Strategies for Their Stimulation in the Human Gut. Frontiers in Microbiology 7, 979, (2016).

22 Brown, J. et al. Structure of the fungal $\beta$-glucan-binding immune receptor dectin-1: Implications for function. Protein Science 16, 1042-1052, (2007).

23 Brown, G. D. Innate antifungal immunity: the key role of phagocytes. Annual Review of Immunology 29 1-21, (2011).

24 Ashida, H., Nishiumi, S. \& Fukuda, I. An update on the dietary ligands of the AhR. Expert Opinion on Drug Metabolism \& Toxicology 4, 1429-1447, (2008).

25 Fukuda, I., Mukai, R., Kawase, M., Yoshida, K.-i. \& Ashida, H. Interaction between the aryl hydrocarbon receptor and its antagonists, flavonoids. Biochemical and Biophysical Research Communications 359 822-827, (2007).

26 Medjakovic, S., Mueller, M. \& Jungbauer, A. Potential Health-modulating Effects of Isoflavones and Metabolites via Activation of PPAR and AhR. Nutrients 2, 241-279, (2010).

27 Busbee, P. B., Rouse, M., Nagarkatti, M. \& Nagarkatti, P. S. Use of natural AhR ligands as potential therapeutic modalities against inflammatory disorders. Nutrition Reviews 71, 353-369, (2013).

28 Jin, U. H. et al. Structure-Dependent Modulation of Aryl Hydrocarbon Receptor-Mediated Activities by Flavones. Toxicological Sciences, (2018).

29 van der Hee, B. et al. Optimized procedures for generating an enhanced, near physiological 2D culture system from porcine intestinal organoids. Stem Cell Research 28, 165-171, (2018).

30 Windey, K., De Preter, V. \& Verbeke, K. Relevance of protein fermentation to gut health. Molecular Nutrition \& Food Research 56, 184-196, (2012).

31 Diether, N. E. \& Willing, B. P. Microbial fermentation of dietary protein: An important factor in dietmicrobe-host interaction. Microorganisms 7, 19, (2019).

32 Bjeldanes, L. F., Kim, J.-Y., Grose, K. R., Bartholomew, J. C. \& Bradfield, C. A. Aromatic hydrocarbon responsiveness-receptor agonists generated from indole-3-carbinol in vitro and in vivo: comparisons with 2, 3, 7, 8-tetrachlorodibenzo-p-dioxin. Proceedings of the National Academy of Sciences 88, 9543 9547, (1991).

33 Maskell, I. E. Nutritional aspects of feeding rapeseed products to rats and pigs. PhD dissertation, University of Newcastle upon Tyne, (1990).

34 Hahn, M. E. The aryl hydrocarbon receptor: a comparative perspective. Comparative Biochemistry and Physiology Part C: Pharmacology, Toxicology and Endocrinology 121, 23-53, (1998).

35 Hahn, M. E. Aryl hydrocarbon receptors: diversity and evolution. Chemico-biological Interactions 141 131-160, (2002)

36 Hahn, M. E., Karchner, S. I., Shapiro, M. A. \& Perera, S. A. Molecular evolution of two vertebrate aryl hydrocarbon (dioxin) receptors (AHR1 and AHR2) and the PAS family. Proceedings of the National Academy of Sciences 94, 13743-13748, (1997). 
37 Murray, I. A. \& Perdew, G. H. Ligand activation of the Ah receptor contributes to gastrointestinal homeostasis. Current opinion in toxicology 2, 15-23, (2017).

38 Murray, I. A., Patterson, A. D. \& Perdew, G. H. Aryl hydrocarbon receptor ligands in cancer: friend and foe. Nature Reviews Cancer 14, 801, (2014).

39 Flaveny, C. A., Murray, I. A. \& Perdew, G. H. Differential gene regulation by the human and mouse aryl hydrocarbon receptor. Toxicological Sciences 114, 217-225, (2009).

40 Ramadoss, P. \& Perdew, G. H. Use of 2-azido-3-[125I] iodo-7, 8-dibromodibenzo-p-dioxin as a probe to determine the relative ligand affinity of human versus mouse aryl hydrocarbon receptor in cultured cells. Molecular Pharmacology 66, 129-136, (2004).

41 Lamas, B., Natividad, J. M. \& Sokol, H. Aryl hydrocarbon receptor and intestinal immunity. Mucosal Immunology 11, 1024-1038, (2018).

42 Hur, S. J., Lim, B. O., Decker, E. A. \& McClements, D. J. In vitro human digestion models for food applications. Food Chemistry 125, 1-12, (2011).

43 Minekus, M. et al. A standardised static in vitro digestion method suitable for food-an international consensus. Food \& function 5, 1113-1124, (2014).

44 Rungapamestry, V., Duncan, A. J., Fuller, Z. \& Ratcliffe, B. Effect of cooking brassica vegetables on the subsequent hydrolysis and metabolic fate of glucosinolates. Proceedings of the Nutrition Society 66, 6981, (2007).

45 de Lavergne, M. D., Derks, J. A., Ketel, E. C., de Wijk, R. A. \& Stieger, M. Eating behaviour explains differences between individuals in dynamic texture perception of sausages. Food Quality and Preference 41, 189-200, (2015).

46 Ulleberg, E. K. et al. Human gastrointestinal juices intended for use in in vitro digestion models. Food Digestion 2, 52-61, (2011).

47 Shapiro, T. A., Fahey, J. W., Wade, K. L., Stephenson, K. K. \& Talalay, P. Chemoprotective glucosinolates and isothiocyanates of broccoli sprouts: metabolism and excretion in humans. Cancer Epidemiology and Prevention Biomarkers 10, 501-508, (2001).

48 Van de Wiele, T., Van den Abbeele, P., Ossieur, W., Possemiers, S. \& Marzorati, M. in The Impact of Food Bioactives on Health: in vitro and ex vivo models (eds Kitty Verhoeckx et al.) 305-317 (Springer International Publishing, 2015).

49 Aguirre, M., Ramiro-Garcia, J., Koenen, M. E. \& Venema, K. To pool or not to pool? Impact of the use of individual and pooled fecal samples for in vitro fermentation studies. Journal of Microbiological Methods 107, 1-7, (2014).

50 Arumugam, M. et al. Enterotypes of the human gut microbiome. Nature 473, 174, (2011).

51 Knights, D. et al. Rethinking "enterotypes". Cell Host \& Microbe 16, 433-437, (2014).

52 Aguirre, M. et al. Evaluation of an optimal preparation of human standardized fecal inocula for in vitro fermentation studies. Journal of Microbiological Methods 117, 78-84, (2015).

53 Aguirre, M. \& Venema, K. Challenges in simulating the human gut for understanding the role of the microbiota in obesity. Beneficial Microbes 8, 31-53, (2017).

54 Kovatcheva-Datchary, P. et al. Linking phylogenetic identities of bacteria to starch fermentation in an in vitro model of the large intestine by RNA-based stable isotope probing. Environmental Microbiology 11, 914-926, (2009).

55 Van den Abbeele, P., Venema, K., Van de Wiele, T., Verstraete, W. \& Possemiers, S. Different Human Gut Models Reveal the Distinct Fermentation Patterns of Arabinoxylan versus Inulin. Journal of Agricultural and Food Chemistry 61, 9819-9827, (2013). 
56 Venema, K., van Nuenen, M. H., van den Heuvel, E. G., Pool, W. \& van der Vossen, J. M. The effect of lactulose on the composition of the intestinal microbiota and short-chain fatty acid production in human volunteers and a computer-controlled model of the proximal large intestine. Microbial Ecology in Health and Disease 15, 94-105, (2003).

57 Mayer, S. et al. C-Type Lectin Receptor (CLR)-Fc Fusion Proteins As Tools to Screen for Novel CLR/ Bacteria Interactions: An Exemplary Study on Preselected Campylobacter jejuni Isolates. Frontiers in Immunology 9, 213, (2018).

58 de Waard, P. W. et al. A human intervention study with foods containing natural Ah-receptor agonists does not significantly show AhR-mediated effects as measured in blood cells and urine. Chemicobiological interactions 176, 19-29, (2008). 



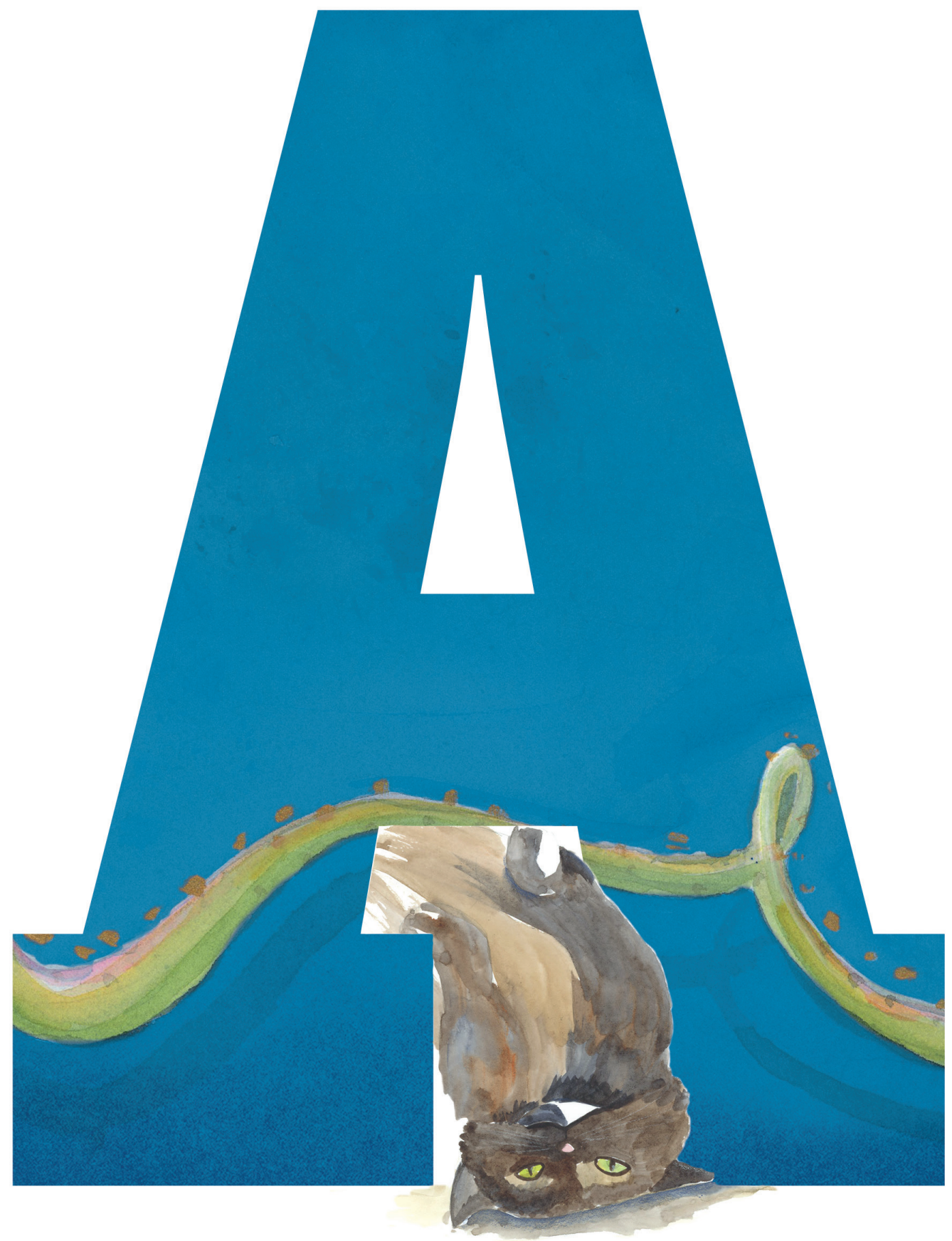


Appendix

Summary 



\section{Summary}

Recent insights have shown that there is a triangular relationship between the diet, immune system and intestinal microbiome, in which the gut microbiota is a substantial contributor to human metabolism and health. A better understanding of the relationship between dietary components, including food processing, and the activity of metabolites produced by the gut microbiota on the host, is an essential step in designing food that benefits human health. The nutrient composition of a diet is obviously of importance to health, but the way food is designed also plays an important role in how much reaches the colon, where it is metabolised by the microbiota. Food design, both industrial food processing and domestic food preparation, can therefore be a tool to influence the nutrients that reach the lower gut and impact on intestinal health. However, more insight is needed into the modulatory effect of digestion and microbial fermentation on the interactions between dietary components and the gut immune system. Therefore, this thesis describes the effects of different dietary components (fibre, tryptophan, glucosinolates and polyphenols) on immune modulation, mainly focussing on activation of the Aryl hydrocarbon Receptor (AhR), during human digestion and fermentation. The results presented in this thesis give insight into the effects of food preparation and microbial fermentation on the potential health properties of food compounds.

Chapter 2 describes the ability of the dietary fibre arabinan to bind to human dendritic cells, and studies the effects of a (food) matrix during fermentation of arabinan containing sugar beet and apple. Dietary fibres such as pectin-derived arabinans are fermented in the colon resulting in bacterial production of short-chain fatty acids (SCFAs), which play a role in gut homeostasis. Additionally, linear arabinan (LA) originating from sugar beet has been reported to strongly activate mouse dendritic cells in a Spleen Tyrosine Kinase (SYK)-dependent manner, suggesting involvement of ITAM-containing C-type lectin receptors (CLR). The aim of this study was to investigate whether LA was immune-stimulatory for human dendritic cells (DCs) and to identify the CLR involved. Purified LPS-free LA bound specifically to human DCs, in a calcium dependent manner, with involvement of SYK. Stimulation of human DCs with LA resulted in strong induction of IL-6, IL-10 and TNF $\alpha$ and these responses were reduced after SYK-inhibition. Using different approached, we ruled out the involvement of Dectin-1, Dectin-2, DC-SIGN, Mincle and the Mannose Receptor in immune activation by LA. Additionally, we fermented sugar beet, apple and sugar beet pectin with human faecal microbiota in vitro to investigate the potential biological relevance of pectin fermentation and immune activity of the glycans in vivo. Filtered supernatants from sugar beet and apple fermentation increased production of IL-6 and TNFa in BMDCs from TLR4/2 knockout mice. However, fermentation of isolated sugar beet pectin only had a small effect on the immune stimulatory activity compared to sugar beet, suggesting an important role for the natural matrix. In conclusion, LA is immune-stimulatory for human DC s in a calcium and SYK-dependent manner, which suggests involvement of a CLR other than the CLRs targeted in this study. 
Besides the immune activation by dietary fibres showed in Chapter 2, many dietary phytochemicals have been reported to promote gut health. Specific dietary phytochemicals, such as luteolin, as well as specific microbial metabolites of tryptophan are ligands of the Aryl hydrocarbon Receptor (AhR), which plays a role in immunity and homeostasis of the gut barrier. In Chapter 3, the effects of oregano, containing the polyphenol luteolin, on AhR activation during in vitro fermentation were studied. We studied the fate of luteolin during colonic fermentation and the contribution of tryptophan metabolites to AhR activity in different parts of the colon. Several polyphenols were screened for AhR activation and oregano, containing the ligand luteolin, was added to batch cultures of human microbiota from the distal colon. Luteolin was rapidly metabolised, with no measurable increase in AhR activity. In the second experiment using the Simulator of the Human Intestinal Microbial Ecosystem (SHIME), not all luteolin was metabolized in the ascending colon, but disappeared rapidly in the transverse colon. The greatest AhR activity was due to microbiota-derived metabolites of tryptophan, particularly in the descending colon. Luteolin in food was rapidly metabolized in the transverse colon. We concluded that tryptophan metabolism by the microbiota in the colon contributes substantially to the pool of lumen metabolites that can activate the AhR.

As a continuation, Chapter 4 describes the importance of microbial metabolites from tryptophan on AhR activation during in vitro fermentation. The gut microbiome has been shown to influence metabolic pathways in mice and humans. Some microbial metabolites of tryptophan are known AhR agonists. The aim of this study was to assess the impact of tryptophan supplementation on the formation of tryptophan metabolites, AhR activation and microbiota composition in the SHIME. AhR activation, microbial composition, and tryptophan metabolites were compared during high tryptophan supplementation and control and wash-out periods. During tryptophan supplementation, the concentration of several tryptophan metabolites was increased compared to the control and wash-out period, but AhR activation by fermenter supernatant was significantly decreased. This was due to the higher levels of tryptophan, which was found to be an antagonist of AhR signalling. Tryptophan supplementation induced most microbial changes in the transverse colon including increased relative abundance of Lactobacillus, which are known to produce AhR agonists by metabolising tryptophan. We conclude that tryptophan supplementation leads to increased formation of AhR agonists in the colon. Although tryptophan itself was found to be an antagonist of AhR activation its impact on AhR activation in vivo is unknown due to uptake and transport by intestinal epithelial cells.

In Chapter 5, broccoli derived AhR ligands were investigated. Broccoli is rich in glucosinolates, which can be converted upon chewing and processing into various metabolites, including AhR ligands. In this study, the effects of temperature, cooking method (steaming versus boiling), gastric $\mathrm{pH}$ and further digestion of broccoli on AhR activation were investigated in vitro and in ileostomy subjects. For the in vitro study, broccoli florets were divided into different treatment 
groups: raw, steamed and boiled followed by an in vitro digestion with different gastric $\mathrm{pH}$. In the in vivo ileostomy study, subjects received a broccoli soup or a broccoli soup plus myrosinase source. AhR activation was measured in both in vitro and in vivo samples by using HepG2-Lucia ${ }^{\text {TM }}$ AhR reporter cells. Cooking broccoli reduced the AhR activation measured after gastric digestion in vitro, but gastric $\mathrm{pH}$ did not have an effect. Indole AhR ligands were not detected or detected at very low levels both after intestinal in vitro digestion and in the ileostomy samples, which resulted in no AhR activation. AhR activation by glucosinolate derivatives present in broccoli is modulated by several factors including cooking method, cooking time and digestion. Broccoli consumption resulted in AhR activation after the oral and gastric phase in vitro. In contrast, after the small intestinal phase of both in vitro and in vivo samples, no AhR activation was found. This suggests that the evaluation of the relevance of dietary glucosinolates for AhR modulation in the gut cannot prescind from the way broccoli is processed and that broccoli consumption does not necessarily produce substantial amounts of AhR ligands in the large intestine.

Finally, Chapter $\mathbf{6}$ discusses the results of the different research chapters, as well as some limitations in the methods used and future research opportunities. 


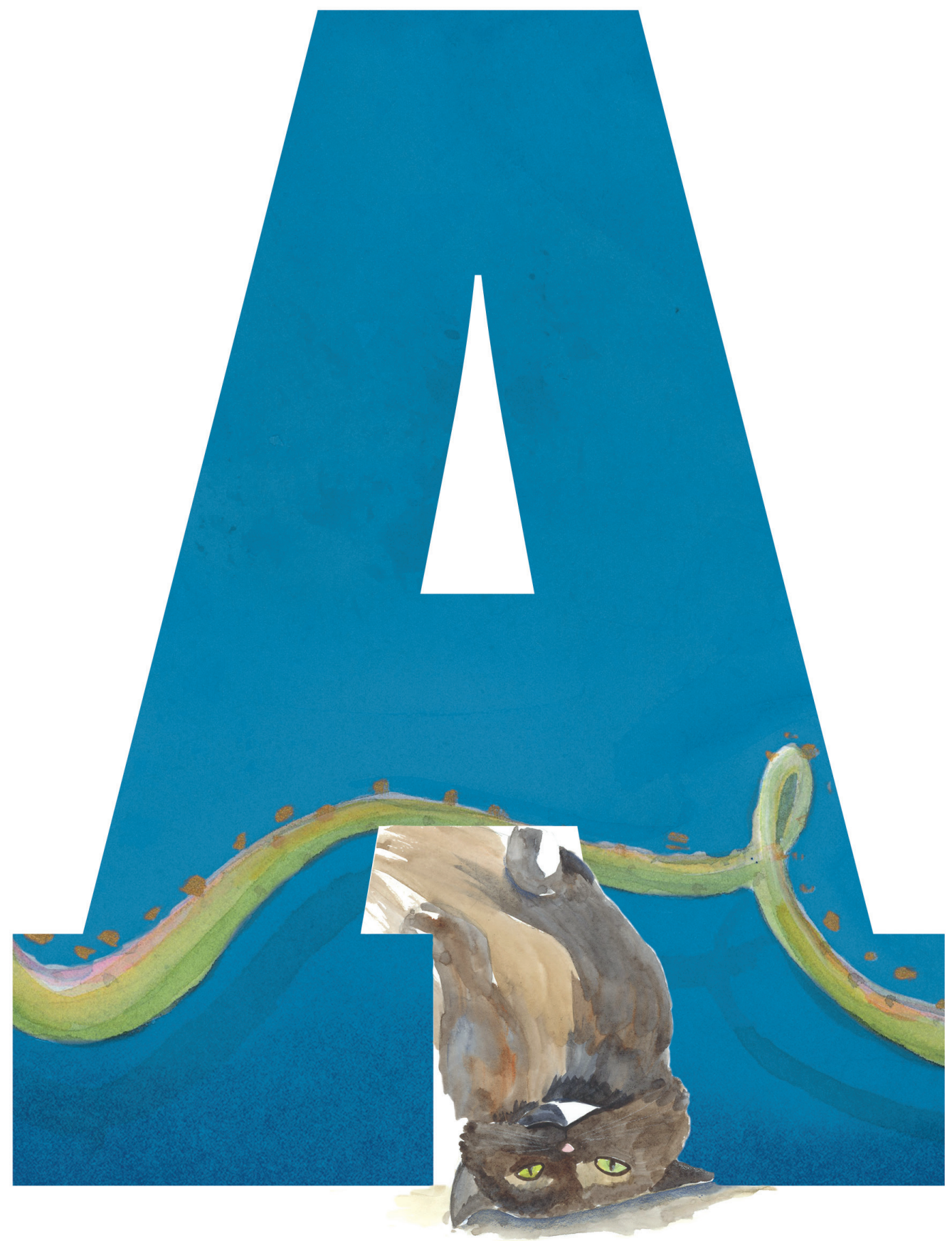




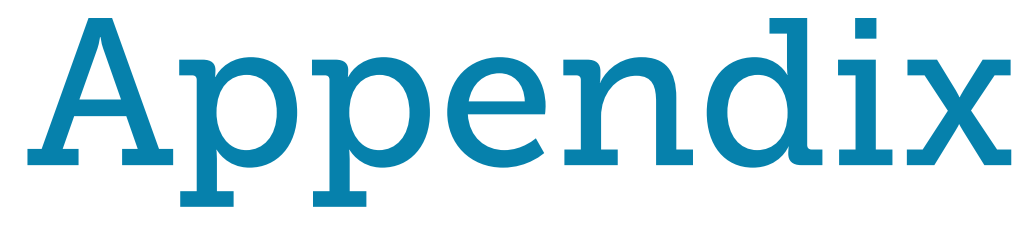

Acknowledgements 



\section{Acknowledgements}

As known, a PhD is not done by one individual. I got help from many others, and I would like to thank them in this acknowledgements section.

First of all, Vincenzo and Jerry, thank you for being my promotors and believing in me. I had a great time working in both of your groups and without you this would not have been possible. You wrote the first VLAG research proposal together and I had the privilege to be the PhD candidate involved. Also Graduate School VLAG, many thanks for funding my research! Vincenzo, thank you for your humour, weird moments and out of the box thinking. You have the quality to bring people together and believing in their qualities. I really appreciate that, thank you! Jerry, your humour and stories were a great pleasure to listen to. Besides, I learned a lot from your writing skills and immunological knowledge. Thank you!

Then, my co-promotors, Edoardo and Linda. Thank you for your 'daily' supervision. Edoardo, I could always just burst into your office with any question and whenever I had a graph ready you were so eager to see the results. Your positive mind-set, even when experiments were majorly failing, was of great value for me. Thank you! Linda, als enige andere Nederlandse vrouw in het team. Bedankt voor de momentjes om even mijn ei kwijt te kunnen, al dan niet werkgerelateerd. Je altijd kritische blik zorgde ervoor dat ik ook kritisch bleef en tot een beter resultaat kwam. Dankjewel!

I would also like to thank the people that I collaborated with in several parts of my research. First of all, I would like to thank ProDigest, in special Beatriz Guimarães and Pieter van den Abbeele, for my 3 months stay in Belgium learning the ins and outs of the SHIME and giving me very useful tips for my research. The SHIME turned out to be of major importance during my research and although there were some smelly accidents, it was a very useful tool. Secondly, prof. Theo Geijtenbeek and Dr. Joris Sprokholt (AMC). Thank you for your hospitality during my stay in Amsterdam for experiments. It was nice to work in another environment and I learned a lot from your immunological techniques. Also many thanks to Ben van den Broek (FBR), for your HPAEC analysis of my apple samples. Last, I want to thank Adil Bouslim and Jeroen Peters for their help with the Biacore experiment in RIKILT.

Special thanks to my hard working students: Jorn, Carien, Luc, Wolf, Maaike and Rudan. It was a great pleasure supervising you all with your thesis and I learned a lot from you. I hope you will become great researchers and have an amazing future career! Thanks to the technicians in the lab: Erik, Geert, Charlotte, Xandra, Frans, Mike (all from FQD) and Nico and Anja (HMI). Your lab-knowledge is worth gold! Ontzettend bedankt voor jullie hulp tijdens mijn onderzoek, in het bijzonder Erik: Dankjewel voor alle lange SHIME sampling dagen. Ik vond het erg fijn om met je samen te werken en ook bedankt voor je humor en de lachmomenten in het lab! 
Ook heel belangrijk: Lieve secretaresses Kimberley (FQD), Loes (HMI), Corine en Lysanne (FQD). Dank voor alles wat jullie voor mij geregeld hebben. Als jullie afwezig waren was er altijd lichte paniek in de groep. Jullie bijdrage aan een goed functionerende groep mag nooit onderschat worden! In het bijzonder, Kimberley, vanaf dag 1 dat ik je ontmoette hadden we zo'n goede klik. Bedankt dat je naast je functie ook mijn vriendin bent, de gekke dansjes in kantoor, de moeilijke momentjes, en je altijd zo vrolijke persoonlijkheid! Loes, ook jij bent altijd positief en je lost alles op. Bedankt voor de gekke skype-chat berichtjes en je oplossingen voor de vaak lastige financiële zaken tussen de verschillende afdelingen.

Next, I cannot forget my colleagues. In FQD, thank you for the nice (not so) flexible office: Femke, Teresa, Annelies, Evita, Arianne and Renske. I think we drunk the WUR out of tea and some people might be surprised how I managed to finish my PhD with all our chatting and laughing. We are just very efficient! Thank you so much for the great time. Also all the other people from FQD, thank you for the nice lunches, PhD trips, coffee talks and Karaoke evenings. Thanks to the lovely and many (ex)PhDs: Valentina, Isabelle, Andrijana, Ruben, Elsa, Daylan, Ayusta, Ana Maria, Mostafa, Dario, Sara, Sine, Alim, Lucia, James, Ita, Ling, Hannah, Chunyue, Moheb, Onu, Lucia, James, Michele, Fabiola, Sydney, Li, Zhan, Zongyao and all the others that make FQD such an amazing group! My 'temporal' FQD colleagues: Domenico, Annachiara, Jialu, Francesca and Lucia, thank you so much for the great times we had together. Thanks to all the FQD staff members, in special Ruud, Teresa and Bea, it was so nice that you joined our PhD trip(s)!

I had the privilege to be working in two departments, so of course I would also like to thank my $\mathrm{HMI}$ colleagues. In special my 'PhD sister' Nuning, thank you for being my friend and the nice talks in good and bad times. I am so proud of you, we both did it! It is an honour to be able to defend my thesis on the same day as you and we will have a great celebration party together! To the rest of my HMI family: Loes, Raka, Nico, Anja, Ellen, Sam, Marcela, Bart, Simon, Edoardo, Isabela, Jori, Marith, Berdien, Laura, Blanca, Michiel, Peter, Joyce, Alex, Jos, Stephane, Simen, Saartje, Avis, Tim and Femke, thank you for the great $\mathrm{HMI}$ atmosphere in both the lab and during dinners, Sinterklaas and other social activities, I enjoyed every second of it!

Ik wil ook graag mijn 'onofficiële collega's' van FBR bedanken: Renata, Coen, Shanna, Harry, Juriaan, Dianne en Matthijs. Bedankt voor de spontane gang gesprekjes die vaak, naast gezellig, wetenschappelijk heel waardevol bleken te zijn!

Marie-Louise, Annelies, Arianne, Evita and Thomas: thank you for your last, very valuable, spelling check of my thesis chapters! 
Uiteraard wil ik ook mijn vriendinnen bedanken. Elske en Hanneke, als je elkaar al meer dan 20 jaar kent, dan zijn woorden misschien niet nodig. Toch wil ik jullie hier bedanken dat jullie er altijd voor me zijn, en ik ben er ook graag voor jullie! Mijn studievriendinnen: Anne-Marieke, Cindy, Fleur, Esther en Sabine. Bedankt voor jullie vriendschappen, ook al zijn sommige op grote afstand. Het is altijd fijn om elkaar te zien en zelfs na lange tijd voelt het alsof het gisteren was. Jullie steun en interesse in mijn werk waardeer ik echt, dankjewel! Emmy, bedankt voor de super gezellige ritjes van en naar België, als jij bij mij in de auto zat leek het toch altijd net iets sneller te gaan ;-). Ook bedankt voor mijn lieve dansdames: Sofie, Meta, Ellen, Lieke, Linda en Linda, Marjon, Magida en Diane. De gezelligheid op de dinsdagavond tijdens modern ga ik heel erg missen! Het was fijn om een momentje in de week te hebben om even te ontspannen en dankzij jullie heb ik dat kunnen doen.

Mijn paranimfen Cindy en papa: bedankt dat jullie mijn paranimfen wilden zijn. Het is fijn om jullie als steun bij me te hebben op deze bijzondere dag!

Bedankt ook voor mijn lieve familie: In nagedachtenis van mijn beide opa's en oma's, Elzelien en Reny, Jan Willem en Ellen en Linda, voor de gezellige (kerst)avondjes en etentjes. Carpe Diem! Ook mijn Belgische schoonfamilie: Magda en Erwin, Eric en Ann, Jurgen en Fiona. Bedankt voor jullie warme welkom in België. Jullie hebben deze 'Hollandse' moeiteloos geaccepteerd in jullie leven en laten me thuis voelen, bedankt!

Lieve papa en mama: jullie hebben me geleerd om altijd te vertrouwen op mezelf en kritisch te blijven. Dank jullie wel daarvoor. Jullie onvoorwaardelijke steun en liefde is niet in woorden uit te drukken. Ik hou van jullie. David en Thomas, mijn lieve broers, bedankt voor jullie interesse in mijn werk, de facetime gesprekken en appjes. Nu is jullie kleine zusje Dr.!

Als laatste, lieve Dimitri: Bedankt voor al je liefde en steun, de tijd die je me gegund hebt om nog een paar jaar langer in Wageningen te zijn om een PhD te doen. Onze gezamenlijke dierenliefde met 'dierentuin' aan beestjes en je nuchtere kijk op de wereld houdt me met beide voetjes aan de grond. Bedankt voor je fantastische kookkunsten en onze avondjes 'ontspannings bingewatching.' Bedankt om elke keer weer in de auto te stappen en 2.5 uur naar Wageningen te rijden voor mij. Nu begint ons nieuwe hoofdstuk, ik kijk er naar uit! 


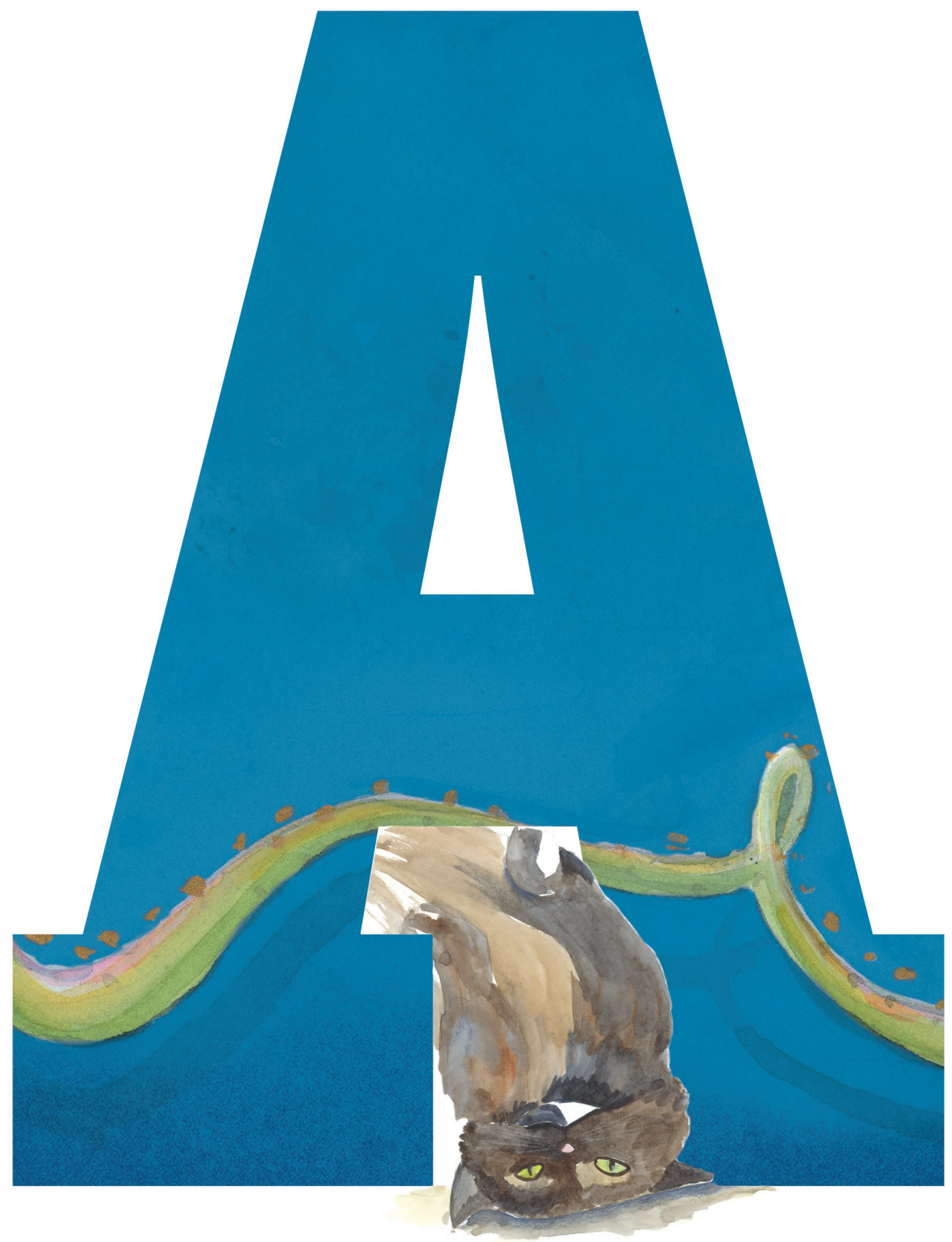




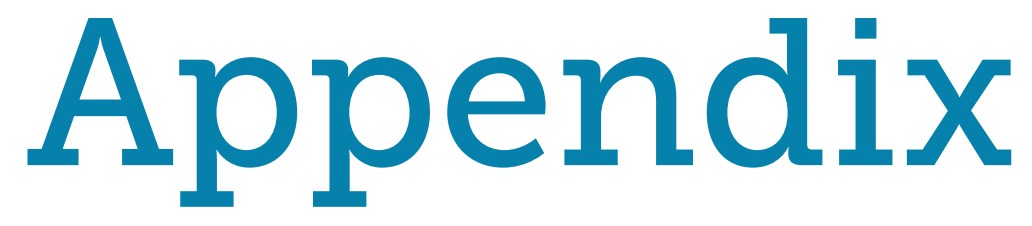

About the author 



\section{About the author}

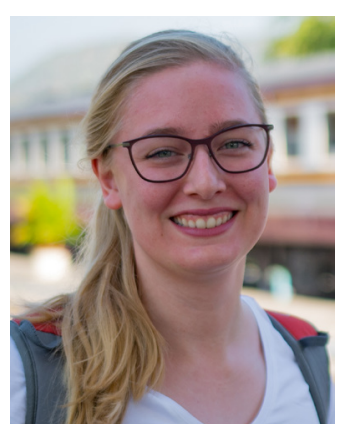

Jonna Koper was born on 15 February 1991 in Tilburg, The Netherlands. She obtained her BSc degree Animal Sciences from Wageningen University in 2012, with a thesis entitled 'the neural and hormonal regulation in satiety feeling in pigs' at the chair group of Adaptation Physiology (ADP) and a minor 'Food, Intestinal homeostasis and disease'.

She continued with the MSc Animal Sciences at Wageningen University and obtained her Research Master degree in 2014, with a double specialisation in both Cell Biology and Immunology (CBI) and Adaptation Physiology (ADP). For her first MSc thesis, she studied the immunemodulating properties of chitin and chitosan, under the supervision of Prof. H. Wichers and Ir. S. Bastiaan-Net. For her second MSc thesis, she studied the influence of environmental conditions on broiler welfare during transport to slaughterhouses, under supervision of Dr. L. Jacobs and Dr. J.E. Bolhuis, at the Institute of Agricultural and Fisheries Research (ILVO) in Ghent, Belgium. After completion of the MSc Research Master Cluster, she was nominated for the Tjeerd de Jong Award and awarded with a money price for scientific development.

After graduation, Jonna worked at the Betasteunpunt Wageningen to develop an immunology practical for biology class in high schools. In September 2015, she started her PhD in the chair groups Food Quality \& Design and Host-Microbe Interactomics and studied immunomodulatory food compounds during in vitro fermentation, with a focus on activation of the Aryl hydrocarbon Receptor. 


\section{Overview of completed training activities}

\section{Discipline specific activities \\ Courses}

Summer Course Glycoscienses, VLAG, Groningen, NL, $2016^{1}$

Training period at other laboratory, ProDigest, Gent, BE, 2016

Advanced food analysis, VLAG, Wageningen, NL, 2017

The Intestinal Microbiome and Diet in Human and Animal Health, VLAG, Wageningen, NL, $2017^{1}$

Healthy food design, VLAG, Wageningen, NL, 2018

\section{Conferences}

Symposium Folia Orthica - Food and hypersensitivity, Atrium Innovations, Amersfoort, NL, 2015 17th Gut Day, Gut Flora Foundation, Rotterdam, NL, 2015

PhD symposium 'Diversity in Science', WUR, Wageningen, NL, 2016

MKA referee evening, Food - Healthy life!, Maastricht University, Maastricht, NL, $2016^{2}$

Seminar TWIN-SHIME, Shared research facilities WUR, Wageningen, NL, $2017^{2}$

Conference of Food Digestion, COST INFOGEST, Rennes, FR, $2017^{1}$

Symposium Folia Orthica - Personalized Nutrition, Atrium Innovations, Amersfoort, NL, 2017

CarboHealth symposium, Carbohydrate Competence Centre, Zwolle, NL, 2017

7th International Dietary Fibre Conference, ICC/TNO/WUR, Rotterdam, NL, $2018^{1}$

The Aryl Hydrocarbon Receptor meeting, Biomedicale Paris Descartes, Paris, FR, $2018^{1}$

VLAG seminar PhD project, VLAG, Wageningen, NL, $2018^{2}$

32nd EFFost International Conference, EFFoST/IUFoST/Elsevier, Nantes, FR, 2018 1,2

Gut Day 2018, Gut Flora Foundation, Wageningen, NL, $2018^{1}$

Seminar 'Integration experimental and theoretical approaches in immunology', WIAS-VLAG, Wageningen, NL, 2019 


\section{General courses}

VLAG PhD week, VLAG, Soest, NL, 2016

PhD Competence Assessment, WGS, Wageningen, NL, 2016

Reviewing a scientific paper, WGS, Wageningen, NL, 2016

Essentials of scientific writing and presenting, WGS, Wageningen, NL, 2016

Introduction to R, VLAG, Wageningen, NL, 2017

Applied Statistics, VLAG, Wageningen, NL, 2017

Scientific Artwork - Vector graphics and images, WUR library, Wageningen, NL, 2017

Philosophy and Ethics of Food Science and Technology, VLAG, Wageningen, NL, 2018

Career assessment, WGS/ Meijer en Meijaard, Wageningen, NL, 2019

Critical thinking and argumentation skills, WGS, Wageningen, NL, 2019

\section{Optionals}

PhD study tour, FQD, Italy, $2016^{2}$

PhD study tour, FQD, Australia, 2018 1,2

Organizing PhD study tour, FQD, Italy, 2016

Meeting and colloquia, FQD, 2015 - $2019^{2}$

Weekly labmeetings, HMI, 2015 - $2019^{2}$

${ }^{1}$ poster presentation

${ }^{2}$ oral presentation

VLAG: Graduate School for Nutrition, Food Technology, Agrobiotechnology and Health Sciences WGS: Wageningen Graduate School

WUR: Wageningen University \& Research

WIAS: Wageningen Institute of Animal Sciences

FQD: Food Quality and Design

HMI: Host-Microbe Interactomics 
The research described in this thesis was financially supported by Graduate school VLAG.

Financial support from Wageningen University for printing this thesis is gratefully acknowledged.

Cover design and layout: (C) evelienjagtman.com

Printed by Ridderprint, www.ridderprint.nl 